\title{
Liner Evaluation for Uranium Mill Tailings: Final Report
}

Compiled by

J. L. Buelt

September 1983

Prepared for the U.S. Department of Energy under Contract DE-AC06-76RLO 1830

Pacific Northwest Laboratory Operated for the U.S. Department of Energy by Battelle Memorial Institute 


\title{
DISCLAIMER
}

This report was prepared as an account of work sponsored by an agency of the United States Government. Neither the United States Government nor any agency thereof, nor any of their employees, makes any warranty, express or implied, or assumes any legal liability or responsibility for the accuracy, completeness, or usefulness of any information, apparatus, product, or process disclosed, or represents that its use would not infringe privately owned rights. Reference herein to any specific commercial product, process, or service by trade name, trademark, manufacturer, or otherwise, does not necessarily constitute or imply its endorsement, recommendation, or favoring by the United States Government or any agency thereof. The views and opinions of authors expressed herein do not necessarily state or reflect those of the United States Government or any agency thereof.

\author{
PACIFIC NORTHWEST LABORATORY \\ operated by \\ BATTELLE \\ for the \\ UNITED STATES DEPARTMENT OF ENERGY \\ under Contract DE-AC06-76RLO 1830
}

\begin{tabular}{|c|c|}
\hline \multicolumn{2}{|c|}{$\begin{array}{l}\text { Printed in the United States of America } \\
\text { Available from } \\
\text { National Technical Information Service } \\
\text { United States Department of Commerce } \\
5285 \text { Port Royal Road } \\
\text { Springfield, Virginia } 22161\end{array}$} \\
\hline \multicolumn{2}{|c|}{$\begin{array}{l}\text { NTIS Price Codes } \\
\text { Microfiche A01 }\end{array}$} \\
\hline \multicolumn{2}{|c|}{ Printed Copy } \\
\hline Pages & $\begin{array}{l}\text { Price } \\
\text { Codes }\end{array}$ \\
\hline $001-025$ & $\mathrm{~A} 02$ \\
\hline $026-050$ & $\mathrm{~A} 03$ \\
\hline $051-075$ & $\mathrm{AOS}_{4}$ \\
\hline $076-100$ & A05 \\
\hline $101-125$ & $\mathrm{~A} 06$ \\
\hline $126-150$ & $A 07$ \\
\hline $151-175$ & $A O B$ \\
\hline $176-200$ & A09 \\
\hline $201-225$ & $A 010$ \\
\hline $226-250$ & A011 \\
\hline $251-275$ & A012 \\
\hline $276-300$ & $\mathrm{~A} 013$ \\
\hline
\end{tabular}


LINER EVALUATION FOR URANIUM MILL TAILINGS:

FINAL REPORT

Compiled by:

J. L. Buelt

Contributors:

S. M. Barnes

M. E. Dodson

V. Q. Hale

W. J. Martin

J. F. Relyea

D. J. Silviera

September 1983

Prepared for the U.S. Department of Energy under Contract DE-ACO6-76RL0 1830

Pacific Northwest Laboratory

Richland, Washington 99352 

SUMMARY

The Liner Evaluation for Uranium Mill Tailings Program was conducted to evaluate the need for and performance of prospective lining materials for the long-term management of inactive uranium mill tailings piles. On the basis of program results, two materials have been identified:

- naturat foundation soil amended with $10 \%$ sodium bentonite

- catalytic airblown asphalt membrane.

The study showed that, for most situations, calcareous soils typical of Western U.S. sites adequately buffer tailings leachates and prevent groundwater contamination without additional liner materials or amendments. Although mathematical modeling of disposal sites is recommended on a sitespecific basis, there appears to be no reason to expect significant infiltration through the cover for most Western sites. The major water source through the tailings would be groundwater movement at sites with shallow groundwater tables. Even so, column leaching studies showed that contaminant source terms were reduced to near maximum contaminant levels (MCL's) for drinking water within one or two pore volumes; thus, a limited source term for groundwater contamination exists. At sites where significant groundwater movement or infiltration is expected and the tailings leachates are alkaline, however, the sodium bentonite or asphalt membrane may be necessary.

The Liner Evaluation Program conducted by Pacific Northwest Laboratory (PNL) can be divided into five major categories:

- tailings characterization studies

- pretiminary studies

- liner evaluations by Taboratory tests

- liner evaluations by field tests

- decision tree development. 
The tailings characterization studies included batch and column leaching studies to determine the contaminant source term in the leachate for the laboratory studies. The preliminary studies identified and evaluated various liner materials and selected eight of the most promising for study. The laboratory liner tests screened the eight materials to identify an asphalt and a clay liner for further study and subjected these materials to controlled evaluations to quantify their expected performance lifetime. The asphalt and clay liners were also subjected to evaluations in the field. During the decision tree development, a logical approach was developed through the use of mathematical models to identify the need for and type of liner at a disposal site.

\section{TAILINGS CHARACTERIZATION STUDIES}

The objective of the tailings characterization studies was to identify tailings leachate concentrations for liner exposure and interaction studies in the laboratory. Various tailings samples from Shiprock, New Mexico, Salt Lake City, Utah; and Durango, Colorado, were collected and subjected to $5: 1$ and 100:1 batch leach tests. Because the leach tests were considered inadequate to identify contaminant concentrations as a function of time, column leaching studies were performed on the most highly contaminated batch samples. The columns produced tailings leachate that indicated a limited contaminant source term. Within two pore volumes, contaminant concentrations were near MCL's for drinking waters. These data are interpreted to mean that only contaminants from the first two pore volumes of leachate need be considered when determining liner requirements.

\section{PRELIMINARY STUDIES}

At the beginning of the program, various liners were evaluated by referring to published literature. The types of liners included clays, soil sealants, synthetic geomembranes, asphalts, and concretes. The following materials were identified for laboratory screening:

- catalytic airblown asphalt

- hydraulic asphalt concrete 
- asphalt-rubber membrane

- chlorosulfonated polyethylene

- natural sojl

- soil amended with sodium bentonite

- soil amended wi th Saline Sea]-100(a)

- soil amended with GSR-60. (a)

\section{LABORATORY TESTS}

Each of the materials was subjected to extreme conditions in 0.6-m diameter exposure columns to duplicate field conditions as closely as possible while subjecting them to increased temperature, acidity, oxygen concentrations, and hydrostatic pressure to accelerate aging reactions. Based on the indicated stability, effectiveness as a leachate barrier, and cost, catalytic airblown asphalt and natural soil amended with sodium bentonite were selected for further study.

The catalytic airblown asphalt was subjected to a series of aging tests in the exposure columns under various temperatures and levels of oxygen and acidity. Aging reaction products were analyzed by infrared spectrometry and results indicated that the asphalt was extremely stable. An aging period equivalent to 7 years was measured, yet the extent and penetration of reaction products was so minute that 1000-year liner performance can be expected to be exceeded under uranium mill tailings impoundment conditions.

Natural soils from Clive, Utah, and soils amended with natural sodium bentonite were subjected to material characterization, batch interaction, and column interaction studies. Results showed that the high $\mathrm{CaCO}_{3}$ content, typical of Western sites, provided an adequate buffering capacity and

(a) Saline-Seal 100 and GSR-60 are registered trademarks of the American Colloid Company. 
caused contaminant coprecipitation in the leachates even without bentonite amendments. This behavior was demonstrated in the column interaction studies, which showed that calcareous soils in contact with acidic leachates become plugged due to coprecipitation, producing permeabilities be tween $10^{-9}$ and $10^{-10} \mathrm{~cm} / \mathrm{s}$.

Natural soils in contact wi th alkaline leachates maintained relatively high initial permeability and demonstrated negligible sorptive or buffering characteristics. When amended with sodium bentonite, however, the clay liner permeability decreased with time while in contact with alkaline leachates. Reductions of cationic contaminant concentrations due to sorption were also observed.

\section{FIELD TESTS}

Catalytic airblown asphalt and sodium bentonite amended soit were also evaluated in the field. The materials were placed in a $1.8-\mathrm{m}$ deep pit in a Grand Junction, Colorado, tailings pile in 1981. Each liner occupied half the pit over a $0.3-m$ thick foundation soil, which was instrumented wi th moisture blocks and moisture extraction cups to obtain readings and samples of moisture migrating through the liners. After a 2-year exposure, the asphalt liner had retained its flexible characteristics and shows little degradation when compared with a virgin sample.

Low moisture saturation levels were maintained in the foundation soil because of the impervious asphalt liner. Insufficient asphalt concentrations occurred over a one-square-meter area, which contained small holes (approximately $1 \mathrm{~cm}$ in diameter) that allowed the foundation soil directly beneath it to become saturated. This experience exemplifies the need for proper engineering and inspection practices when applying an asphalt membrane.

The clay liner did not keep the foundation soil dry for more than three months because it is much more permeable and the leachates were not acidic ( $\mathrm{pH}=7$ to 8 ). The beneficia) characteristics of this liner are 
associated with the sorptive properties of the sodium bentonite in the clay. Comparisons of moisture above and below the clay liner showed that contaminant concentrations were reduced as the leachate passed through the liner. Analysis of clay liner cores after the 2-year exposure verified that contaminants are not mobile through this material.

\section{DECISION TREE DEYELOPMENT}

With the permeabilities, buffering, and sorptive characteristics of the two liner materials identified, a logical approach was developed to combine these data with hydrologic models and contaminant source terms from the tailings. This approach, called a decision tree, is a step-by-step sequence that can be used to jdentify the need for and type of liner at a particular disposal site. Models such as the TRUST code and UNSATID can be used to determine the long-term (1000-year) behavior of the impoundment as it pertains to groundwater quality. 



\section{ACKHOWLEDGENENTS}

This report is a compilation of published and unpublished data and conclusions developed throughout the course of the progran. The Program Manager wishes to recognize the following personnel for their technical and interpretive contributions:
S. M. Barnes
W. C. Buchmiller
M. E. Dodson
K. E. Eljason
V. Q. Hale
S. D. Harris
W. J. Martin
J. F. Relyea
D. J. Silviera 
CONTENTS

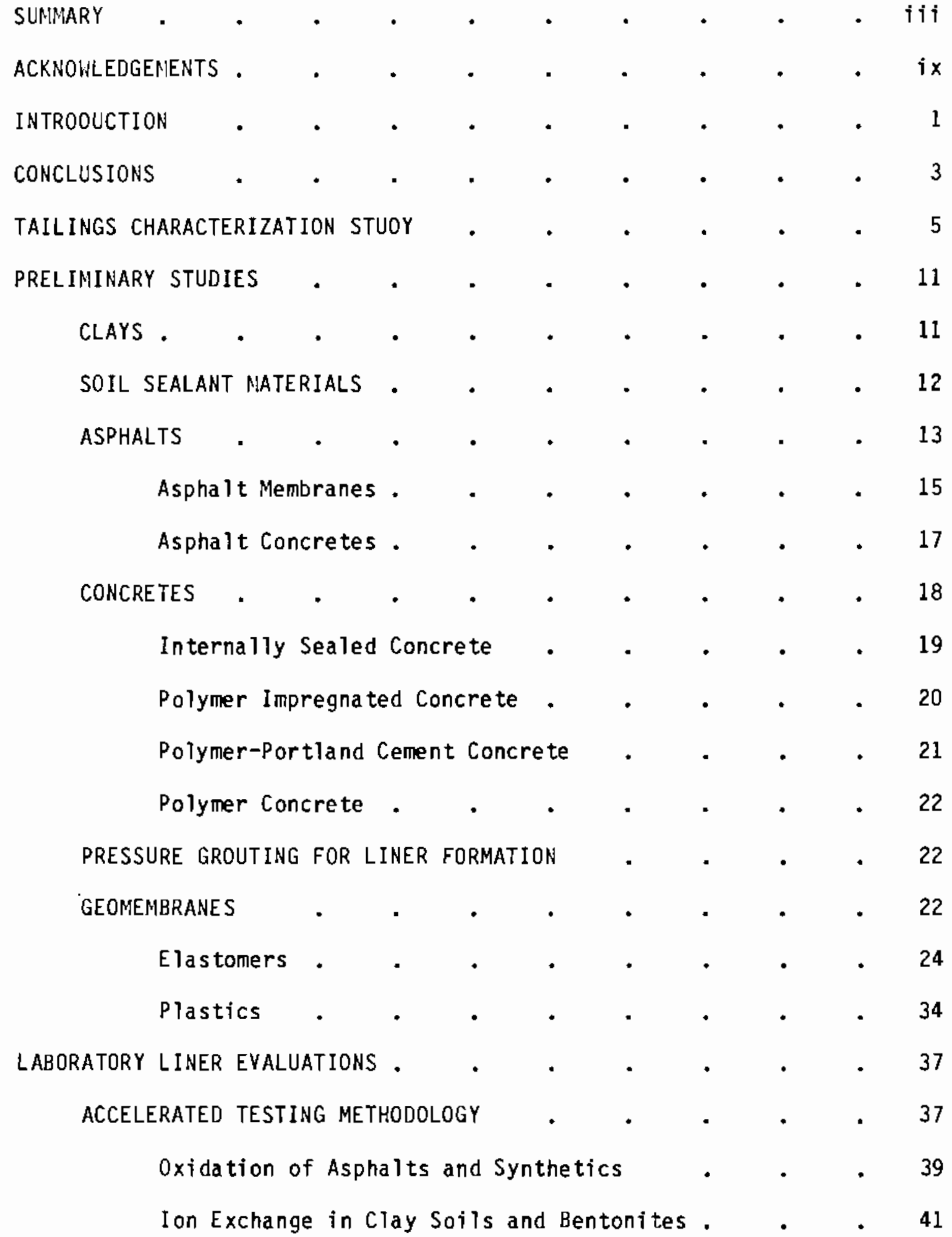




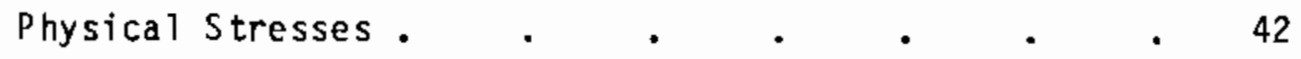

Radiation Exposure $\quad . \quad$. $\quad . \quad$. $\quad . \quad 433$

EXPOSURE COLUNN DESIGN

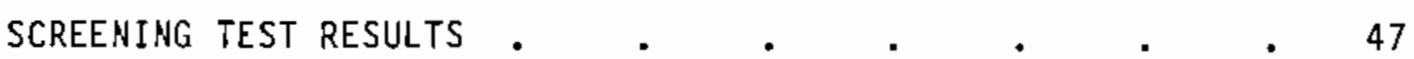

Asphalt and Synthetic Liners . . . . . 49

Asphalt-Rubber Liner . . . . . . 52

CTay Liners . $\quad . \quad$. $\quad . \quad$. 55

Sodium Bentonite Liner . . . . . . 58

Saline Seal 100 and GSR-60 Liners . . . . 58

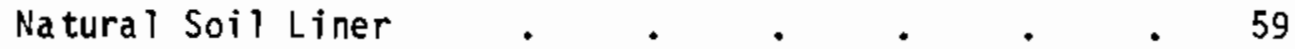

Liner Costs . $\quad . \quad$. $\quad . \quad$. 59

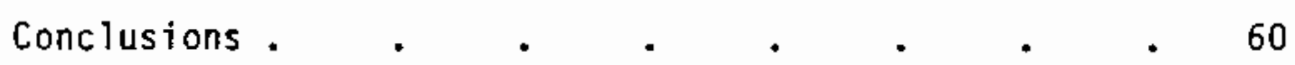

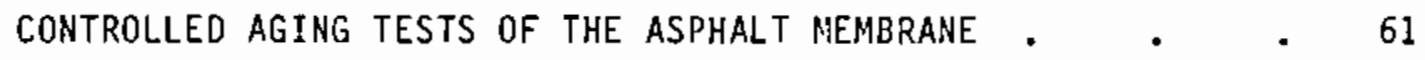

Exposure Conditions . . . . . . . 61

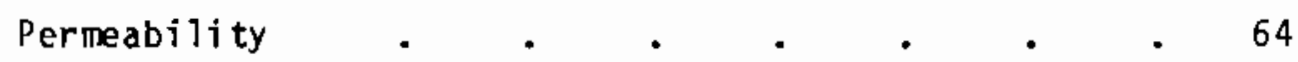

Analytical Results . $\quad$. $\quad . \quad . \quad . \quad 66$

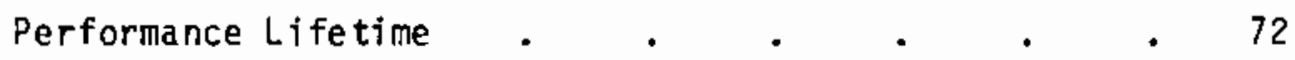

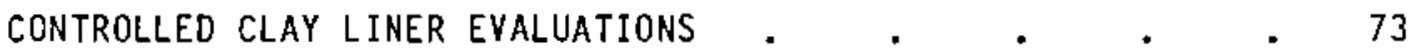

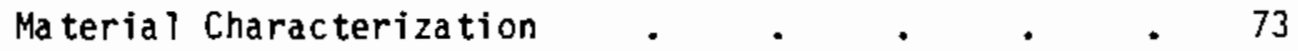

Leachate Interactions with Soil and Liner Materials . 75

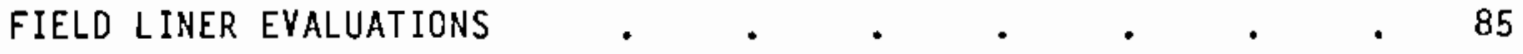

TEST DESCRIPTION

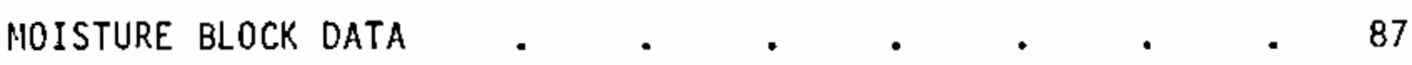

LEACHATE SAMPLE ANALYSIS $\quad$ • . . . . . . . 93

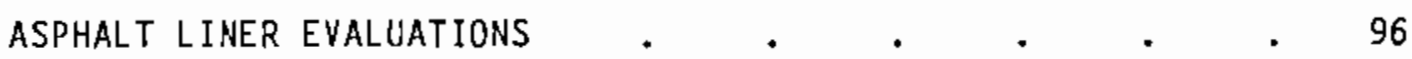




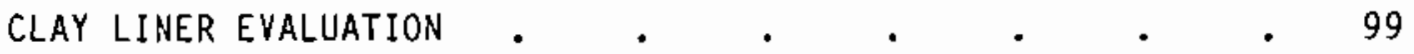

Sediment Analysis . $\quad . \quad$. $\quad . \quad$. 100

Solution Analysis. $. \quad . \quad . \quad . \quad . \quad . \quad 101$

RESULTS AND DISCUSSION .

PHYSICAL CHARACTERISTICS $\quad$. $\quad . \quad$.

Clay Liner Attenuation Characteristics . . . 106

CONCLUSIONS ON FIELD LINER EVALUATIONS • • • • • . 114

OECISION TREE FOR LINER EVALUATION • • • • • • • • 115

SITE CHARACTERIZATIONS • • • • • • • • 115

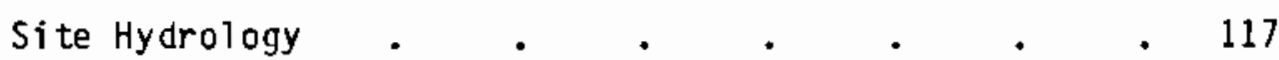

Taflings Characterization . . . . . . 121

Contaminant Attenuation . . . . . . . 122

WATER MOVEMENT WITHOUT A LINER • • • • • • • 126

CONTAMINANT TRANSPORT WITHOUT A LINER $\quad$ • • • 127

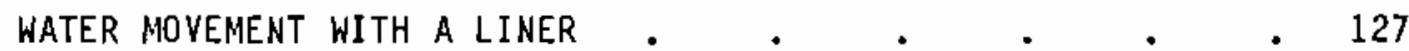

CONTAMINANT TRANSPORT WITH A LINER • • • • • • 128

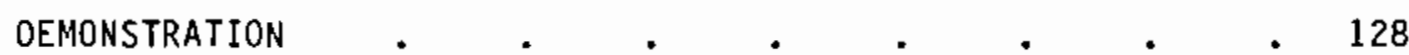

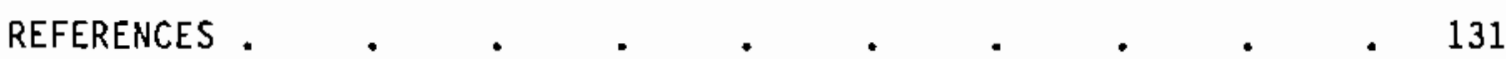

APPENDIX A - PERMEABILITY TEST DATA $\quad$. . . . . A.1

APPENDIX B - ASPHALT LINER SPECIFICATIONS $\quad$ • $\quad$ • $\quad$ • $\quad$ B.1

APPENOIX C- EXPOSURE CONDITIONS, PERMEABILITIES, AND

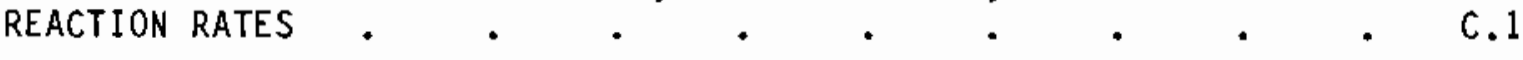




\section{FIGURES}

1 Sample Sites on the Salt Lake City Vitro Tajlings Pile . 5

2 Sample Sites on the Durango Tailings Pile . . . . 6

3 Sample Sites on the Shiprock Tailings Pile . . . . 6

4 Gegcentrations of Radioactive Elements ${ }^{230} \mathrm{Th},{ }^{238} \mathrm{U}$, and Ra in Tailings Leachates. . . . . . . 10

5 Concentrations of Nonradioactive Hazardous Elements in Tailings Leachates. $. \quad . \quad . \quad . \quad . \quad . \quad$. 10

6 Typical Disposal Site for Predicting Subsidence . $\quad$ - $\quad 42$

7 Accelerated Aging Laboratory Apparatus $\quad$. $\quad$ * $\quad$. 44

8 Construction Details of Liner Exposure Columns . . . 45

9 Permeability Performance of Asphalt and Hypalon

10 Permeability Performance of Clay-Amended and Natural Soil Liners During the Laboratory Testing

Period . . . . . . . . . . . . 48

11 Oxygen Pressure to which Asphalt and Synthetic Liners Were Exposed During Testing. . . . . . . 50

12 Acidity Concentration During Testing of Asphalt and Synthetic Liners. . . . . . . . . 51

13 Exposure Temperature of Aspha1t and Synthetic Liners During Testing . . . . . . . . . 52

14 Pressure History During Testing of Clay Liners . . $\quad 56$

15 Acidity Concentration During Testing of Clay Liners . . 57

16 Fractional Factorial Matrix . . . . . . . . . . 62

17 Permeability of Asphalt Liners at Normal, Intermediate,

18 Absorption Bands of 0.C.T., Paraplast, and Asphalt . . 67

19 Reaction Rates at Normal and Highly Acidic Conditions $\quad$ - 69 
I

. 
20 Effect of Temperature on Reaction Rates at High Oxygen

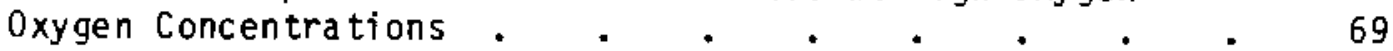

21 Effect of Oxygen on Reaction Rates . . . . . 70

22 Reaction Rates at Intermediate Exposure Conditions . . 70

23 Titration Curves for Soil \#2 Using $\mathrm{HCL}$, and

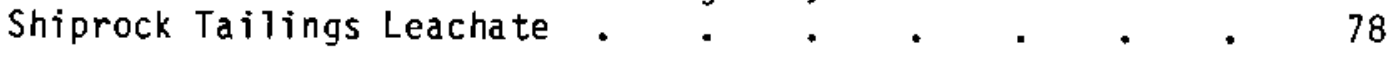

24 Sa turated Hydraulic Conductivity $\mathrm{K}, \mathrm{cm} / \mathrm{s}$, as a Function of Durango Tailings Leachate Pore Volumes Passing Through the Soil Columns. . . .

25 Sa turated Hydraulic Conductivity $\mathrm{K}, \mathrm{cm} / \mathrm{sec}$, as a

Function of Durango Tailings Leachate Pore Volumes

Passing Through the Soil Columns . . . . . . 82

26 Saturated Hydraulic Conductivity $\mathrm{k}, \mathrm{cm} / \mathrm{sec}$, as a

Function of Durango Tailings Leachate Pore Volumes

Passing Through the Soil Columns . . . . . . 83

27 Cross-Section of Tailings Pit for Liner Evaluation

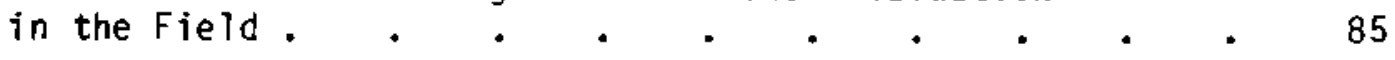

28 Locations of Moisture Blocks and Extraction Cups . . 87

29 Spray Application of Asphalt Membrane . . . . 88

30 Rototilling of Bentonite into Foundation Soil . . . 89

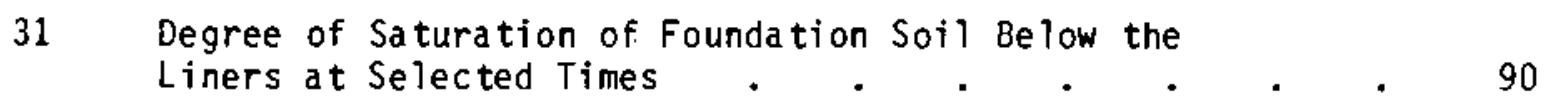

Degree of Saturation Under Asphalt and Clay Liners at
Selected Locations

33 Water Level in Tailings Above Asphalt and Clay Liners . 93

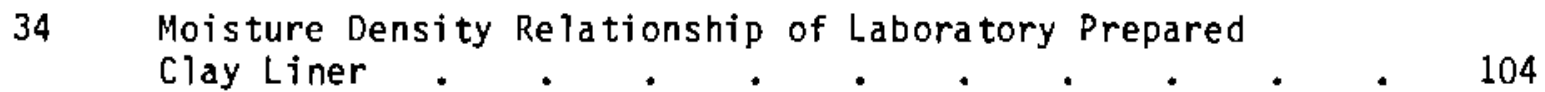

35 Particle Size Distribution of Laboratory Prepared

Clay Liner . . . . . . . . . . . . 104

36 Particle Size Distribution of Field Test Clay Liner . . 105

37 Decision Tree Evaluation of Inactive Uranium

Processing Sites for Liner Requirements . . . . 116

38 Water Balance for a Hypothetical Uranium Mill

Tajlings Disposal Site . . . . . . . . 118 


\section{TABLES}

1 Analytical Results From Tailings at Salt Lake, Durango, and Shiprock Sites Using 100:1 Leachate-to-Sample Ratio . 8

2 Comparison of Column and Batch Leach Tests:

Chemical Composition of Tailings Leachates . . . . $\quad 9$

3 Representative Soil Sealants . . . . . . . . . 14

4 Internally Sealed Concrete Specification . . . . . 19

5 Summary of Effects of Immersion of Polymeric Membrane

Liners in Leachate for Eight Months. . . . . 25

6 Effect of Immersing Liner and Sealing Materials in

Hazardous Waste--A Preliminary Study . . . . . 26-27

7 Properties of Polymeric Liner Membranes Installed

as Barriers . . . . . . . . . . 28-29

8 Effect of Gamma Radiation on Physical Properties . $\quad 32$

9 Effect on Properties(a) of Polymeric Membrane Liners

of One Year of Exposure to Leachate from Simulated

Sanitary Landfills . . . . . . . . . 33

10 Anticipated Field Liner Permeabilities . . . . . 49

11 Costs of the Candidate Liner Materials . . . . 60

12 Simulated Tailings Leachate Composition . . . . . 63

13 Characteristics of Clive, Utah, Soil Samples . . . 74

14 Comparison of Synthetic Leachate Compositions Before

and After Contact With Soit $\$ 6$ in $5: 1$ Solution to

Solid Ratio Batch Test. . . . . . . . . 76

15 Buffering Capacities of Clive, Utah, Soils and

Liner Material . . . . . . . . . 77

16 Leachate Sample Analysis . . . . . . . . . 94

Comparison of Field, Virgin, and Laboratory-Aged
Asphalt Liners..

18 Field Sample Description . . . . . . . . 100 
19 Physical Characteristics of Field and Laboratory Clay Liners . $\quad . \quad$. $\quad . \quad$. . . . 105

20 Core No. 5 Saturated Paste $\mathrm{pH}$, Eh, and Ec Data with Respect to Depth . . . . . . . . 106

21 1:1 Extract pH, Eh, and Ec Data with Respect to Depth . 107

22 X-Ray Fluorescence Data Indicating Concentrations of Solid Material for Uncontacted and Leached Tailings and Laboratory Clay Liner . . . . . . . 108

23 X-Ray Fluorescence Data for Core No. 5 Subsamples . . 110

24 Chemical Analysis of Tailings and Uncontacted

Liner Material: 1:1 Extracts . . . . . . . 111

25 Chemical Analysis of Core Subsamples: 1:1 Extracts . . 113 


\section{INTRODUCTION}

A number of inactive uranium tailings piles have accumulated from milling operations throughout the United States. In 1978, Congress passed Public Law 95-604, which requires disposal of the 24 sites that are the responsibility of the U.S. Department of Energy (OOE). In response, DOE created the Uranium Mill Tailings Remedial Action Progan (UMTRAP); one of the program activities was to develop and evaluate technologies for disposal of the piles in conformance with regulatory criteria established by the U.S. Environmental Protection Agency (EPA). The disposal technologies required, among other things, a program to evaluate the need for and type of liners for long-term (1000-year) protection of the groundwater. For this reason, UMTRAP created the Liner Evaluation for Uranium Mill Tailings Program conducted by Pacific Northwest Laboratory (PNL). This final report is a compilation of all relevant results and conclusions of the PNL liner program.

The PNL liner evaluation program was established late in FY-1980 and was completed in FY-1983. It consisted of five major areas of activity:

- tailings characterization studies

- preliminary liner studies

- liner evaluations by laboratory tests

- liner evaluations by field tests

- decision tree development.

The selected approach was to determine the long-term performance of two of the most promising liner materials identified through a preliminary literature survey and laboratory screening tests. Evaluation of the two liner materials, a natural soil amended with sodium bentonite and an asphalt membrane, included interaction with various tajlings Teachate compositions identified in the tailings characterization studies and assessment after a 2-year field exposure. The study data can be used in the decision tree, which is a sequence developed to determine the long-tern, need for and performance of a liner under uranium tailings impoundment conditions. 
,

. 


\section{CONCLUSIONS}

Through some of the findings of the PNL liner progran and other UMTRAP programs, it became apparent that the natural retarding properties of disposal site soils are, in most cases, enough to prevent groundwater contamination from mill tailings. Calcareous soils, typical of Western U.S. sites, interact with acidic leachate and cause contaminant coprecipitation as the leachate passes through the foundation soil/tailings interface. Where groundwater movement or infiltration through alkaline tailings exists, however, sodium bentonite amended soils or a catalytic airblown asphalt membrane may be required if the groundwater quality objectives established for the disposal site are exceeded. Laboratory evaluations and field studies identified these two materials as the most promising.

In addition to the above findings, the following major conclusions have been derived from the PNL program activities:

- Contaminant releases from tailings as Teachate passes through them are limited. For example, the column leaching studies showed that concentrations of all radionuclides and chemical contaminants were reduced to near or below maximum contaminant levels (MCL's) for drinking water (Environmental Protection Agency 40 CFR 141) within one or two tailings pore volumes.

- Sodium bentonite amended soits and catalytic airblown asphatt proved to be the most effective materials as leachate and contaminant barriers. They were also among the least expensive of the eight materials selected for testing during the preliminary studies.

- Accelerated aging tests successfully exposed the asphalt membrane to the equivalent of a 7-year exposure over a 3-month period. Reaction rates were extremely 510 and penetration of reaction products was limited to $0.5 \%$ of the total membrane thickness. 
The data indicate that a performance period greater than 1000 years can be expected when this material is exposed to uranium mill tailings inpoundment conditions.

- Analysis of the field-exposed asphalt membrane demonstrated similar penetration of reaction products. After a 2-year exposure, the liner retained its pliable characteristics; however, due to inadequate membrane thickness in a small area, some leakage was noted through $1-\mathrm{cm}$ diameter holes. This experience exemplifies the need for proper engineering and inspection practices.

- The buffering capacity of a highly calcareous soil from Clive, Utah, once considered as a disposal site and chosen for the PNL clay/soit evaluations, was 1100 meq/100 g of soil (i.e., $100 \mathrm{~g}$ of soit without amendments will neutralize up to 11,000 liters of acidic leachate from $\mathrm{pH}=2$ to $\mathrm{pH}=4.5$, which is more than adequate to prevent groundwater contamination).

- Coprecipitation of contaminants from acidic leachates in contact with calcareous soils tends to plug the pores and reduces permeabilities to $10^{-10} \mathrm{~cm} / \mathrm{s}$.

- Calcareous soils in contact with alkaline leachates exhibit no sorptive properties and maintain their relatively high permeabilities uniess amended with $10 \%$ sodium bentonite.

- Although the clay liner installed in the field in contact with neutral pH leachates did not keep its foundation soil dry for more than 3 months, it did reduce contaminant concentrations effectively as the leachate passed through the liner.

- The decision tree developed during this program could be useful in evaluating the need for and type of liner at specific disposal sites. This analytical tool considers site-specific conditions and evaluates the long-term groundwater quality through da ta compiled in the PNL program and from existing hydrologic models. 


\section{TAILINGS CHARACTERIZATION STUDY}

The objective of the characterization study of the Liner Evaluation Program was to determine the chemical properties of tailings at 3 of the 24 DOE tailings sites. In turn, the chemical contents of the tailings were used to determine the conditions under which the effectiveness of the liners would be tested.

Tailings samples were taken from the Salt Lake City, Utah; Durango, Colorado; and Shiprock, New Mexico, sites, which were sampled at the locations indicated in Figures 1, 2, and 3. Each sample was composited over a depth of about $1.8 \mathrm{~m}$. At the time of sampling, a wide range of textures was noted among locations in a single pile, apparently the result of how the slimes and sands settled in the original tailings pond. At Salt Lake City, samples taken for moisture determinations indicated that moisture content correlated with increased fineness of texture.

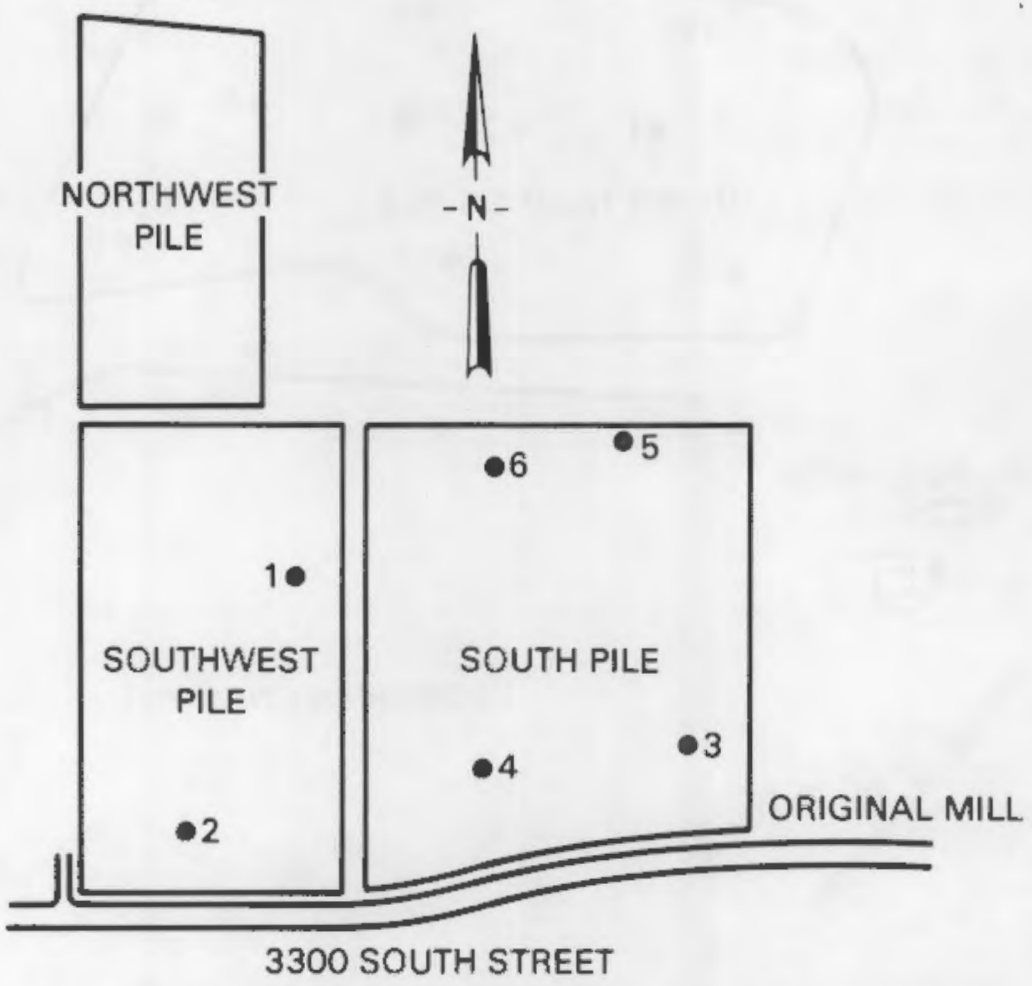

FIGURE 1. Sample Sites on the Salt Lake City Vitro Tailings Pile 


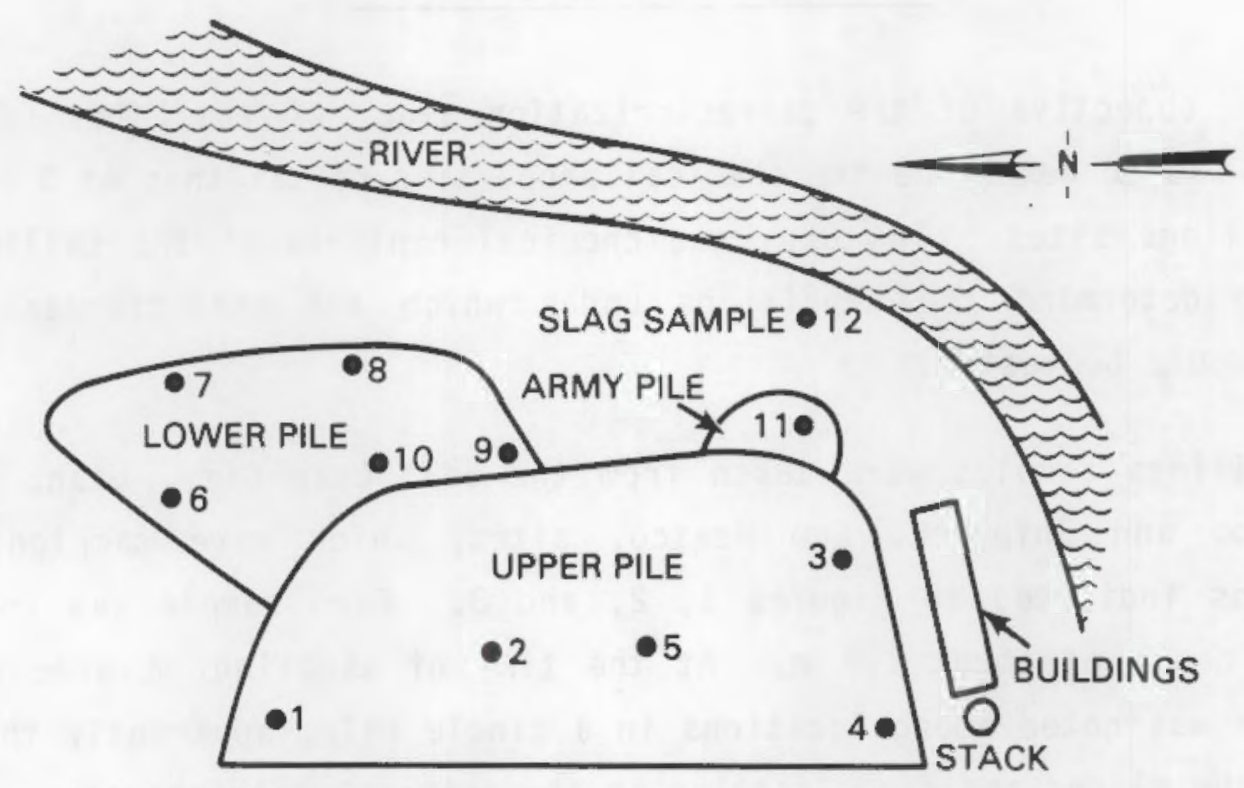

FIGURE 2. Sample Sites on the Durango Tailings Pile

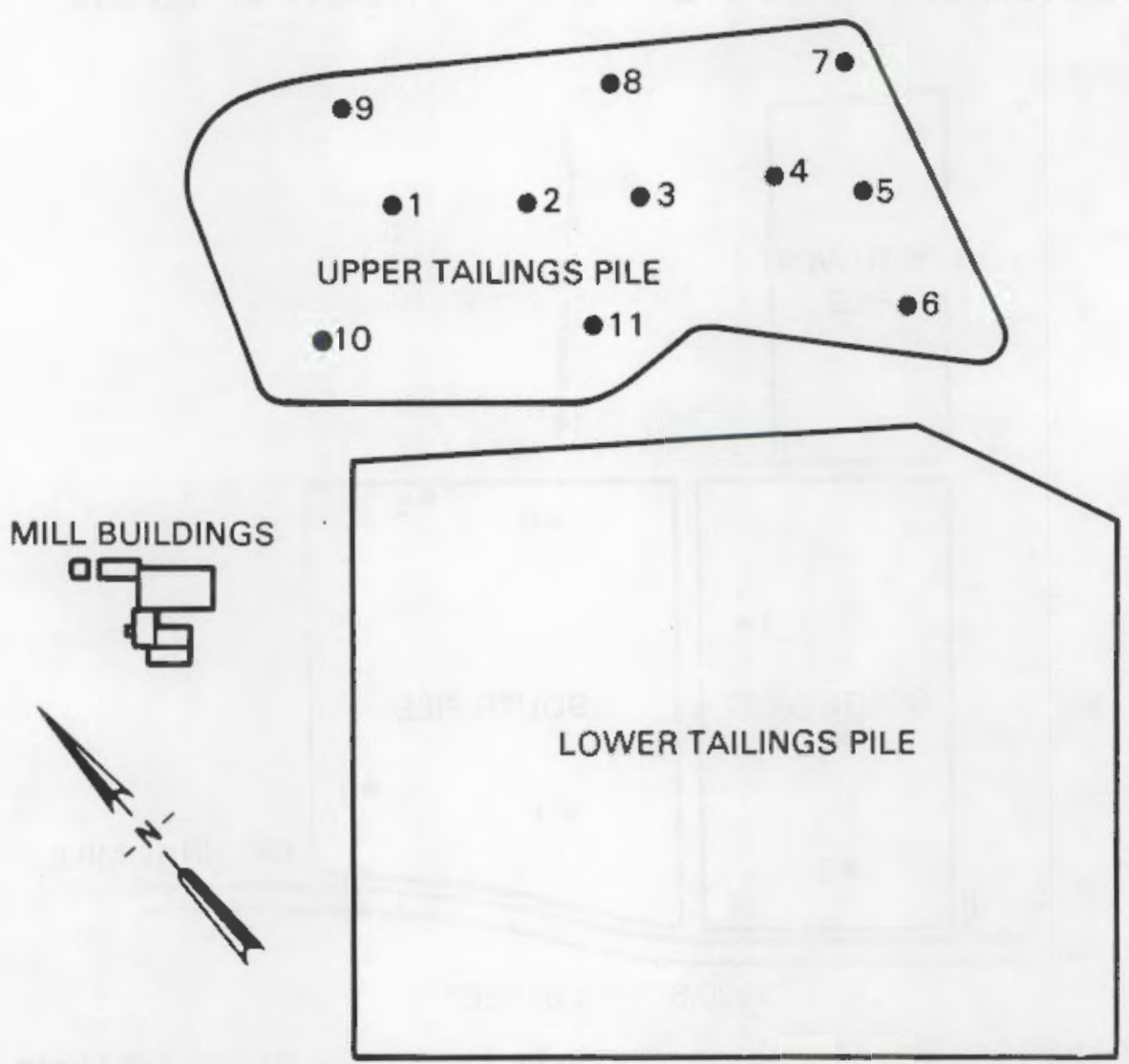

FIGURE 3. Sample Sites on the Shiprock Tailings Pile 
The tailings samples were sealed in buckets and returned to the laboratory, where they were analyzed for cations, anions, hydrocarbons, and radionuclides. Extracts for cation and anion analyses were obtained by shaking tailings with water in a 100 to 1 (by weight) concentration for about 2 hours. Cations were analyzed by inductively coupled argon plasma techniques, the anions by ion chromatography, and the radionuclides by gamma scan. Hydrocarbon concentrations were assumed to be negligible. Analytical results are shown in Table 1 . Although these methods detect and/or quantify a number of elements and ions, only those of special interest to this study are included in the table.

The 100 to 1 leachate samples were used to determine the total quantity of leachable material. To assess the composition of tailings leachate in contact with a liner, the batch leaching technique is adequate to screen a large number of samples but is inadequate to quantify contaminant releases over time. As indicated in recent studies (Relyea, Martin and Colclough 1982), batch leach tests generally underestimate contaminant concentrations that are initially leached from tailings. In fact, batch leaching tests yield little, if any, information on contaminant release rates. (See Table 2 for a comparison of leachate composition from 5 to 1 batch tests and column tests.) Therefore, column leaching tests were conducted to characterize contaminant release rates and concentrations from tailing samples.

Results from column leach tests on tajlings samples have shown that the highest concentrations of contaminants are released in the first or second pore volumes of leachate. As shown in Figure 4, concentrations of radionuclides ${ }^{238} \mathrm{U},{ }^{230} \mathrm{Th}$ and ${ }^{226} \mathrm{Ra}$ decreased by at least an order of magnitude after one pore volume. However, radioactive element concentrations after two pore volumes were still observed to be above the EPA proposed MCL's for all three tailings samples (USEPA 40 CFR 141). Contaminant concentrations of $\mathrm{Al}, \mathrm{As}, \mathrm{Cd}, \mathrm{Cr}, \mathrm{Cu}, \mathrm{Fe}, \mathrm{Mg}, \mathrm{Mn}, \mathrm{Mo}, \mathrm{Ni}, \mathrm{P}, \mathrm{SO}_{4}$ and $\mathrm{Zn}$ were also highest in the first increment of leachate from the three tailings samples tested and decreased by an order of magnitude or more at the end of one pore volume. 
TABLE 1. Analytical Results From Tailings at Salt Lake, Durango and Shiprock Sites Using 100:1 Lechate-to-Sample Ratio

\begin{tabular}{l} 
Salt Lake \\
\hline $\mathrm{pH}$ \\
$\mathrm{Al}$ (ppm in taiiings sample) \\
$\mathrm{Ca}(\mathrm{ppm})$ \\
$\mathrm{Fe}(\mathrm{ppm})$ \\
$\mathrm{Mg}(\mathrm{ppm})$ \\
$\mathrm{Na}(\mathrm{ppm})$ \\
$\mathrm{Si}(\mathrm{ppm})$ \\
$\mathrm{SO}_{4}(\mathrm{ppm})$ \\
$\mathrm{NO}_{3}(\mathrm{ppm})$ \\
$\mathrm{Cl}^{(\mathrm{apm})}$ \\
$210_{\mathrm{pb}}(\mathrm{dpm} / \mathrm{g})$ \\
$238_{\mathrm{U}}$ and $234 \mathrm{Th}(\mathrm{dpm} / \mathrm{g})$ \\
$230_{\mathrm{Th}}(\mathrm{dpm} / \mathrm{g})$ \\
$235_{\mathrm{U}}(\mathrm{dpm} / \mathrm{g})$ \\
$226_{\mathrm{Ra}}(\mathrm{dpm} / \mathrm{g})$
\end{tabular}

\begin{tabular}{|c|c|c|c|c|c|}
\hline 1 & 2 & 3 & 4 & 5 & 6 \\
\hline 2.9 & 7.4 & 8.0 & & 6.7 & 5.5 \\
\hline 109 & 26 & 46 & 11 & 14 & 19 \\
\hline \multirow[t]{2}{*}{24,200} & 14,000 & 8,290 & 26,000 & 36,200 & 25,500 \\
\hline & 30 & 23 & 500 & 2 & 3 \\
\hline 62 & 88 & 194 & 400 & 230 & 256 \\
\hline 162 & 290 & 235 & 1,800 & 1,330 & 660 \\
\hline 17 & 238 & 305 & 566 & 153 & 87 \\
\hline 2,400 & 1,670 & 1,670 & 8,140 & 2,720 & 2,230 \\
\hline$<1$ & 26 & 87 & $<1$ & 383 & 310 \\
\hline 6.4 & 12 & 14 & 1,333 & 79 & 15 \\
\hline 2,430 & & 1,370 & & 1,970 & \\
\hline 90 & & 190 & & 49 & \\
\hline 960 & & 620 & & 460 & \\
\hline$<17$ & & 10 & & 29 & \\
\hline 2,270 & & 1,240 & & 2,000 & \\
\hline
\end{tabular}

\begin{tabular}{l} 
Qurango \\
\hline $\mathrm{pH}$ \\
$\mathrm{Al}$ (ppl in tailings sample) \\
$\mathrm{Ca}$ (ppm) \\
$\mathrm{Fe}$ (ppm) \\
$\mathrm{Mg}$ (ppm) \\
$\mathrm{Na}(\mathrm{ppm})$ \\
$\mathrm{Si}(\mathrm{ppm})$ \\
$\mathrm{CO}_{3}$ (ppm- assumed from inorganic \\
$\mathrm{SO}_{4}$ \\
$210_{\mathrm{Pb}}$ \\
$238_{\mathrm{S}}$ and ${ }^{234} \mathrm{Th}$ \\
$230_{\mathrm{Th}}$ \\
$235_{\mathrm{U}}$ \\
$226_{\mathrm{Ra}}$
\end{tabular}

\begin{tabular}{|c|c|c|c|c|c|c|c|c|c|}
\hline 1 & 2 & 3 & 4 & 6 & 7 & 8 & 9 & 10 & 11 \\
\hline 9.9 & 10.0 & 9.8 & 10.0 & 9.4 & 9.5 & 7.1 & 7.5 & 9.6 & 7.0 \\
\hline 32 & 11 & 11 & 17 & 63 & 162 & 6 & 16 & 11 & $<3$ \\
\hline 1,170 & 1,140 & 800 & 1,060 & 375 & 989 & 2,170 & 600 & 860 & 174 \\
\hline 4.1 & -- & 2 & 1 & 23 & 35 & 4 & 6 & -. & 2 \\
\hline 132 & 100 & 18 & 86 & 37 & 68 & -- & 11 & 136 & - \\
\hline 230 & 110 & 116 & 377 & 75 & 112 & 32 & 45 & 368 & 32 \\
\hline 106 & 60 & 130 & 73 & 343 & 500 & 90 & 100 & 198 & 38 \\
\hline 3,500 & 3,060 & 1,600 & 2,500 & 1,590 & 2,000 & 280 & 365 & 3,100 & 280 \\
\hline low & low & low & lan & -. & $\ldots$ & 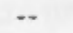 & - & -- & -- \\
\hline & 1,500 & & & 1,530 & & & & & 995 \\
\hline & 575 & & & 275 & & & & & 154 \\
\hline & 2,840 & & & 2,035 & & & & & 1,520 \\
\hline & 27 & & & $<14$ & & & & & 12 \\
\hline & 2,300 & & & 1,940 & & & & & 1,360 \\
\hline
\end{tabular}

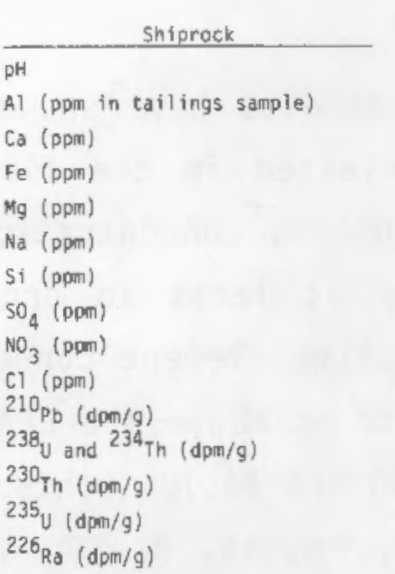

\begin{tabular}{|c|c|c|c|c|c|c|c|c|c|c|}
\hline \multicolumn{11}{|c|}{ Sample Number } \\
\hline IA & 18 & 2 & 3 & 4 & 5 & 6 & 8 & 9 & 10 & II \\
\hline 7.0 & 4.1 & 4.1 & 4.6 & 6.3 & 4.4 & 4.4 & 4.6 & 4.2 & 4.8 & 5.1 \\
\hline 21 & 97 & 367 & 31 & 8 & 99 & 187 & $<3$ & 173 & 7 & 3 \\
\hline 7,790 & 6,300 & 15,500 & 7,220 & 13,500 & 39,700 & 44,600 & 8,950 & 34,400 & 7,290 & 7,230 \\
\hline 7 & 29 & 35 & 3 & 3 & 1 & 6 & 1 & 2 & 2 & \\
\hline$\cdot 182$ & 160 & 320 & 61 & 210 & 466 & 511 & 37 & 183 & 29 & 58 \\
\hline 180 & 340 & 260 & 83 & 232 & 518 & 523 & 34 & 139 & 39 & 144 \\
\hline 185 & 345 & 32 & 51 & 191 & 61 & 69 & 69 & 52 & 37 & 55 \\
\hline 19,000 & 17.600 & 45,200 & 20,900 & 33,300 & 101,300 & 112,500 & 22,100 & 88,400 & 20,000 & 18,800 \\
\hline 33.5 & 13.0 & 10.0 & 4.6 & 21.2 & 37.2 & 34.0 & 6.9 & 2.0 & 2.8 & 15.4 \\
\hline 4.0 & 4.3 & 3.0 & 3.2 & 4.7 & 9.4 & 17.5 & 3.1 & 13.2 & 4.0 & 3.0 \\
\hline 605 & & 1,170 & & & 3,700 & & & & & \\
\hline 25 & & 33 & & & $<23$ & & & & & \\
\hline 340 & & 370 & & & 1,900 & & & & & \\
\hline$<8$ & & 10 & & & 27 & & & & & \\
\hline 510 & & 1,100 & & & 4,025 & & & & & \\
\hline
\end{tabular}

(a) Perfonmed with 1:1 leachate-to-sample ratio. Concentration limited by solubility product. 
TABLE 2. Comparison of Column and Batch Leach Tests: Chemical Composition of Tailings Leachates (Concentrations in $\mathrm{mg} / \mathrm{L}$ )

\begin{tabular}{|c|c|c|c|c|c|c|}
\hline & \multicolumn{2}{|c|}{ Salt Lake } & \multicolumn{2}{|c|}{ Shiprock } & \multicolumn{2}{|c|}{ Durango } \\
\hline & ${\underline{5: 1 ~ \mathrm{Batch}^{(\mathrm{a})}}}^{\left(\mathrm{a}^{2}\right.}$ & Column $^{(b)}$ & 5:1 Batch & Column & 5:1 Batch & Column \\
\hline A 1 & 4.0 & 1200.0 & 66.0 & 2700.0 & 0.6 & 11.0 \\
\hline $\mathrm{Ca}$ & 600.0 & 550.0 & 560.0 & 530.0 & 1.1 & 7.2 \\
\hline Cd & 0.05 & 2.6 & 0.1 & 5.3 & $<0.01$ & 0.15 \\
\hline $\mathrm{Cu}$ & 1.9 & 48.0 & 3.5 & 130.0 & $N D^{(c)}$ & ND \\
\hline $\mathrm{Fe}$ & 6.7 & 1700.0 & 2.4 & 160.0 & ND & 0.17 \\
\hline $\mathrm{Mg}$ & 14.3 & 280.0 & 54.0 & 2100.0 & 7.2 & 17.0 \\
\hline $\mathrm{Mn}$ & 2.2 & 55.0 & 1.5 & 65.0 & ND & 0.02 \\
\hline $\mathrm{Na}$ & 5.5 & 110.0 & 31.5 & 1500.0 & 120.0 & 1900.0 \\
\hline $\mathrm{Ni}$ & 0.6 & 21.0 & 0.3 & 11.0 & ND & ND \\
\hline $\mathrm{Zn}$ & 2.2 & 98.0 & 2.9 & 120.0 & ND & 0.12 \\
\hline $\mathrm{pH}$ & 3.0 & 2.0 & 3.1 & 2.3 & 9.3 & 9.5 \\
\hline
\end{tabular}

(a) The 5:1 batch tests utilize $5 \mathrm{~cm}^{3}$ of distilled water for each gram of tailings.

(b) The volume of effluent collected corresponds to the first 0.20 of 0.27 pore volumes for the leach tests.

(c) ND $=$ Not Detected in the sample.

Concentrations of $\mathrm{As}, \mathrm{Cd}$, and $\mathrm{Cr}$ in leachate from acidic Salt Lake and Shiprock tailings (shown in Figure 5) were near or below the EPA proposed MCL levels after two pore volumes. Similarly, leachate concentrations of As, $\mathrm{Cd}$, and $\mathrm{Cr}$ from alkaline Durango tailings decreased to near or below MCL after one or two pore volumes. $\mathrm{Ba}$ and Pb were below MCL in all samples.

In conclusion, although the batch leaching studies agreed well with the results of Markos and Bush (1981a,b), they did not portray release rates accurately. The column leaching studies demonstrated that contaminant concentrations are markedly reduced after the first two tailings pore volumes of leachate. 


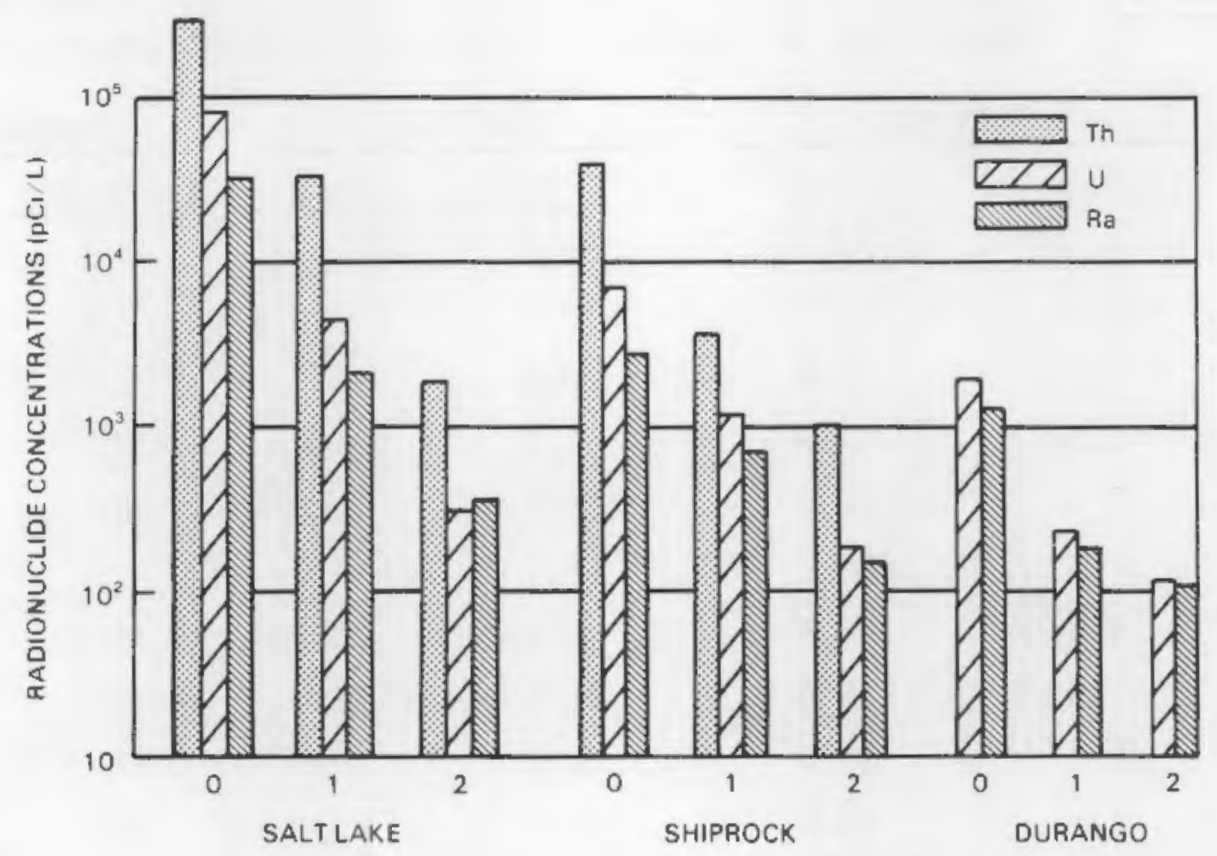

FIGURE 4. Concentrations of Radioactive Elements ${ }^{230} \mathrm{Th},{ }^{238} \mathrm{U}$, and ${ }^{226} \mathrm{Ra}$ in Tailings Leachates. Initial concentrations (first effluent) are labeled 0 . Concentrations at the end of 1 and 2 pore volumes are labeled 1 and 2, respectively.

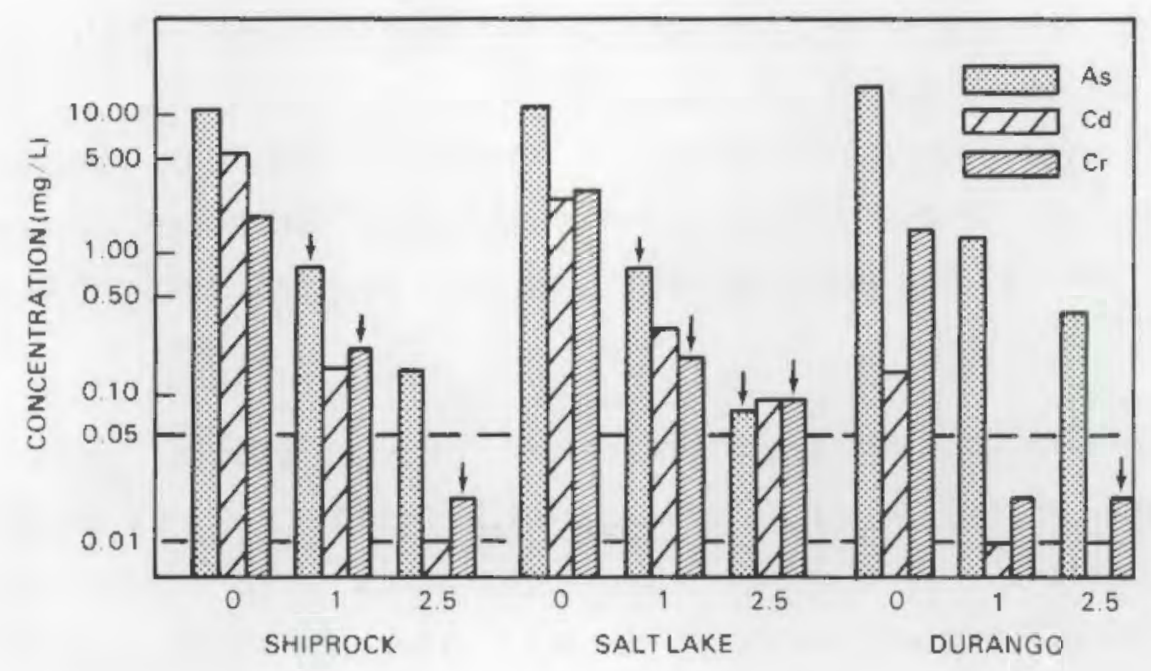

FIGURE 5. Concentrations of Nonradioactive Hazardous Elements in Tailings Leachates. Initial concentrations (first effluent) are labeled 0 . Concentrations at the end of 1 pore volume and $2-1 / 2$ pore volumes are labeled 1 and 2.5, respectively. An arrow on the bar denotes a measured value below detection. 


\section{PRELIMINARY STUDIES}

The preliminary studies were conducted to select eight of the most promising liner materials from those considered for laboratory testing. The studies included a review of published literature and information obtained from industrial contacts. Clays, soil treatments, asphalt membranes, asphalt concretes, grouts and concretes, and polymeric membranes were considered; and the materials were evaluated for long-term effectiveness as a moisture and/or contaminant barrier with cost effectiveness in mind.

\section{CLAYS}

Various clay liners have been installed beneath impoundments containing waste materials. These liners include untreated natural clay minerals and clays artificially saturated with sodium to resist loss of sealing properties when leached by wastes of high ionic strength (Fuller 1981; Griffin and Shimp 1978; Haxo 1976a; Haxo and White 1976b; Lubina, Hovater and McCready 1979; Williams 1978; Shultz and Miklas 1980).

The chemical properties of the waste and the methods used to fill the impoundment influence the choice and design of the liner system. If exposed to natural weathering cycles such as wet/dry and freeze/thaw, a clay liner can lose much of the sealing ability it had when installed (Rollins and Dylla 1970). However, if the clay liner is initially hydrated with fresh water and covered to prevent drying, it can form an effective sea 1.

The sealing property of clay results from its ability to expand when wet. This expansion decreases the pore space of the soil and its permeability. Clay minerals called smectite or montmorillonite are generally responsible for this sealing property. Other clay minerals such as illite and kaolinite also expand when wet, but not as much as montmorillonite.

The ions present on the exchange sites of the clays also influence the amount of shrinking and swelling. In the presence of solutions containing 
high concentrations of dissolved salts, Na in prehydrated clay may be replaced by other ions such as $\mathrm{Ca}$ and $\mathrm{Mg}$, causing the clay to shrink and lose some of its effectiveness as a sealant. Na-saturated montmorillonite, illite, and kaolinite swell more than the same clays saturated with Ca. Clays subject to less swelling have been used as sealants on a limited basis. These clays are affected less by increased concentrations of $\mathrm{Mg}$ or $\mathrm{Ca}$, and the shrinkage caused by drying may also be less. The selection of clay minerals to be used in a liner will depend on availability at specific site locations as well as the characteristics of the leachate within the mill tailings pile.

A special form of montmorillonite, Saline Seal 100, is reported to be resistant to attack by high concentrations of ionic contaminants (Hughes 1974); and this material was selected for the laboratory tests. The Saline Seal 100 clay was mixed with natural soil to determine optimum composition for a clay liner. In addition, natural soils from typical sites and sodium bentonite amendments were studied to compare sealing properties with Saline Seal 100.

One significant advantage of natural clay minerals over geomembrane materials is their low cost (U.S. NRC 1979), especially if they can be obtained close to the disposal site. Another advantage is their self-healing ability. If the liner fails because of minor breaks, the clay will seal itself by filling up the breach in the liner with particles over a period of time. This self-healing ability eliminates problems that can arise if ground settling or other events cause damage to the liner (Hughes $1974)$.

\section{SOIL SEALANT MATERIALS}

Studies have shown that permeability can be reduced by the application of various chemicals that react with the soil. However, most soil sealants have been reported to be affected by freezing/thawing, wetting/drying, reactions with leachates, and leaching of the sealing agent by the waste liquid, as Stewart (1978) discusses below. 
"Most of these materials (sealants) consist of a blend of a highmolecular-weight linear polymer and a crosslinked, swellable polymer of approximately the same molecular weight. The linear polymer's many sorptive sites allow it to sorb to the soil and form a flexible network. The cross-linked polymer is extrudable and can conform to permeability channels in the soil without loss of integrity. In use, the polymer system helps to form a stabilized soil surface of extremely low permeability.

"Formulation of the polymeric soil sealant system depends on its use, and in most instances, it is considered to be proprietary by the marketing company. When used in unfilled impoundments, the polymer is mixed in a low-pH water/acid solution and then sprayed on the earthen surface as a low-viscosity slurry. The low-pH condition allows the polymer to penetrate the surface. Upon subsequent exposure to water, the water-swellable portion swells and becomes locked in place. The linear polymer, being sorptive in this state, attaches to the soil to complete formation of the stable, impermeable surface."

No actual field data are available, but Stewart also discusses sealant limitations, including polymer shrinkage, that affect the seal. Because of these limitations, the sealants identified by Stewart (1978) in Table 3 did not receive further consideration as long-term moisture barriers.

\section{ASPHALTS}

The low water permeability of asphaltic compositions was known to ancient peoples, and the long-term stability of these compositions is evidenced by the survival of several early asphaltic structures (The Asphalt Institute 1976). They are versatile in application and can be alloyed with various materials, creating compounds to meet a range of situations. Asphalt-based compounds can be formulated to resist temperature extremes, to provide water permeability less than $10^{-9} \mathrm{~cm} / \mathrm{s}$ (Hickle 1976), and for a variety of application methods. Asphalts resist most oxidizing acids, except nitric, and attacks from salts and alkalies up to concentrations of $30 \%$ in solution. However, these compounds are

- oxidized by exposure to ultraviolet radition

- sometimes attacked by microbes if not protected by biocides 
- susceptible to subgrade displacement due to poor compaction, freeze/thaw volume changes, and subsidence

- soluble in solutions $>5 \%$ hydrocarbons.

Should further characterization of the tailings leachate reveal significant quantities of the organics used in the solvent extraction process (kerosene, isodecanol, and alanine) (U.S. NRC 1980), asphaltic liners can be protected by the application of a final tar seal coat.

\section{TABLE 3. Representative Soil Sealants (Stewart 1978)}

\begin{tabular}{|c|c|c|}
\hline Sealant & Application & Remarks \\
\hline $\begin{array}{l}\text { Cationic Asphalt } \\
\text { Emulsion }\end{array}$ & Farm Ponds & $\begin{array}{l}\text { Requires } \sim 19,000 \mathrm{~L} / 4,047 \mathrm{~m}^{2}(5000 \mathrm{gal} / \\
\text { acre) dispersed in water. }\end{array}$ \\
\hline $\begin{array}{l}0 \text { il-Soluble } \\
\text { Polymers in } \\
\text { Diesel Fuel }\end{array}$ & Fresh Water & $\begin{array}{l}\text { Injected beneath surface of water } \\
\text { where seepage was occurring. }\end{array}$ \\
\hline $\begin{array}{l}\text { Sodium } \\
\text { Tetraphosphate }\end{array}$ & $\begin{array}{l}\text { Sulfite Liquor } \\
\text { Storage }\end{array}$ & $\begin{array}{l}\text { Dispersant distributed in } 15.2-\mathrm{cm} \\
(6-\mathrm{in} .) \text { layes of soil at } 2.3 \mathrm{~kg} / 9 \mathrm{~m}^{2} \\
\left(5 \mathrm{1b} / 100 \mathrm{ft}^{2}\right) \text {. Careful compaction } \\
\text { rendered soil impervious. }\end{array}$ \\
\hline Sodium Carbonate & Canals & $\begin{array}{l}\text { Wet-dry cycles disrupt water barrier. } \\
\text { Used } 183 \mathrm{~g}(0.41 \mathrm{~b}) \text { of reagent per } \\
0.84 \mathrm{~m}^{2}\left(y \mathrm{~d}^{2}\right) \text { of soil. }\end{array}$ \\
\hline $\begin{array}{l}\text { Lignin Derivatives } \\
\text { Gelled Alum }\end{array}$ & $\begin{array}{l}\text { Desalination } \\
\text { Byproduct Brine }\end{array}$ & $\begin{array}{l}\text { 1\% lignin cost } \$ 3400 \text { per } 4047 \mathrm{~m}^{2} \\
\text { (acre). }\end{array}$ \\
\hline $\begin{array}{l}\text { Carboxyme thy } 1 \\
\text { Cellulose (CMC) } \\
\text { with Alum }\end{array}$ & $\begin{array}{l}\text { Desalination } \\
\text { Byproduct Brine }\end{array}$ & $\begin{array}{l}0.2 \% \text { CMC cost } \$ 2250 \text { per } 4047 \mathrm{~m}^{2} \\
\text { (acre). }\end{array}$ \\
\hline $\begin{array}{l}\text { Petroleum } \\
\text { Emulsions }\end{array}$ & $\begin{array}{l}\text { Desalination } \\
\text { Byproduct Brine }\end{array}$ & $\begin{array}{l}4 \% \text { additive cost } \$ 4400 \text { per } 4047 \mathrm{~m}^{2} \\
\text { (acre). }\end{array}$ \\
\hline Attapulgite Clay & $\begin{array}{l}\text { Desalination } \\
\text { Byproduct Brine }\end{array}$ & $\begin{array}{l}2 \% \text { Zeogel cost } \$ 1000 \text { per } 4047 \mathrm{~m}^{2} \\
\text { (acre). }\end{array}$ \\
\hline $\begin{array}{l}\text { Liquid Elastomeric } \\
\text { Polymer }\end{array}$ & Fresh Water & $\begin{array}{l}\text { Patent discloses several compositions, } \\
\text { including polyure thane elastomers. }\end{array}$ \\
\hline
\end{tabular}


The asphaltic compositions considered in the preliminary studies for tailings impoundment liners included asphalt membranes, asphalt/fabric membranes, asphaltic concretes, and soil asphalts. These compositions are described below.

Asphalt llembranes

\section{Rubberized Asphalt Membranes}

Hot-sprayed membranes are prepared by heating a selected asphalt to $220-240^{\circ} \mathrm{C}$ and applying it to a prepared subgrade with a spray bar system. The selected asphalt must meet the temperature requirements for the application, be flexible at the lowest expected temperature, and be sufficiently viscous at the highest expected temperature to resist flowing from sloped sections (The Asphalt Institute 1976). Because rubber decreases the effect of temperature on asphalt viscosity (Dunning 1975), these temperature requirements can be met by adding small quantities (2-3 wt $)$ of rubber to the asphalt. The elastic properties of rubberized asphalt make this material better suited to conditions, such as subsidence and temperature cycling, expected at tailings repository sites.

Rubberized asphalts are also being developed to prevent crack propagation between layers of airport runways, and they have been used as water barriers beneath highway surfaces. The membranes are placed in two or more layers (lifts) to seal pinholes formed by escaping moisture in the first lift. Typically, the composition is applied at a rate of $7 \mathrm{~L} / \mathrm{m}^{2}$ to achieve a thickness of 5-8 mm. The membranes must be protected by a layer of soil before heavy equipment is operated above the liner surface (Stewart 1978; Middlebrooks et a T. 1978).

\section{Catalytic Airblown Asphalt liembranes}

Catalytic airblown asphalt membranes use high-softening-point asphalts to form a membrane seat. Because this type of liner is designed for applications where it will be exposed to solar heating (e.g., canal linings or roofing applications), high-temperature creep properties are very important. The asphalt is heated and sprayed in place as above, but it requires 
a catalyst to modify the viscosity properties of the asphalt for application.

Low-temperature ductility is also a concern with these asphalts. Haxo and White (1976b) exposed a catalytic asphalt liner to landfill leachate for 1 year and found that the membrane did not deteriorate visibly and absorbed $2.9 \%$ of the leachate. No firm permeability data were reported, but a significant increase in low shear-rate viscosity was noted. Preexposure permeability of catalytic membranes is less than $10^{-9} \mathrm{~cm} / \mathrm{s}$ (Haxo 1976a). Because of the apparent potential of this material, catalytic airblown asphalt was selected for further study.

\section{Aspha1t Emulsion on Fabric}

These membranes are created by placing fabric mats on the subgrade, sewing them together to form a single fabric liner, and applying an asphalt emulsion to seal the fabric. The asphalt is occasionally chip-sealed to provide additional protection from mechanical damage (The Asphalt Institute 1976; Clark et al. 1974). Permeabilities of less than $10^{-9} \mathrm{~cm} / \mathrm{s}$ have been reported for this type of liner (Haxo and White 1976a).

This form of asphaltic membrane has several limitations that affect its suitability for long-term service, and the small amount of asphalt applied is a primary concern. Haxo et al. (1979), Haxo and Whi te (1976a), and Haxo et al. (1977) report testing an $0.8-\mathrm{cm}$ asphalt emulsion/fabric liner. If a hole develops in the asphait and exposes the fabric to potentially corrosive materials or if the fabric is attacked by elements in the soil, an unsupported $0.8-\mathrm{cm}$ asphalt liner would be required to withstand the tensile and shear stresses and hydrostatic pressure of the tailings impoundment. oxidation, and resulting loss of flexibility, of the thin membrane is also a potential failure mechanism. The oxidized asphalt could delaminate from the fabric, resulting in the failure mechanisms outlined above. In addition, the asphalt emulsion can be applied only at temperatures above freezing. Because of these limitations, asphalt emulsion on fabric was excluded from further study. 
Asphalt Concretes

\section{Paving Concre te}

Asphaltic compositions in this category are designed for roadway construction. Typically, paving concrete consists of 4-6 wt\% asphalt; the remainder is carefully graded aggregate. Occasionally, rubber is added to decrease the effect of temperature on the asphait properties. Because these concretes are designed for traffic use, they inciude 6-10 vol\% voids so the concrete can move under pressure exerted by vehicular traffic without cracking. Based on the relatively high void content and the failure of this style of liner reported by Haxo and White (1976a) after exposure to landfill leachage for 1 year, this material received no further consideratjon.

\section{Hydraulic Asphalt Concrete}

Hydraulic concretes are similar to paving concretes; however, asphalt content is increased to 6-10\% (Middlebrooks et a 1.1978 ), voids are reduced to $\langle 4 \%$, and aggregate contains smaller, more rounded rock and mineral fill. Permeabilities to $10^{-10} \mathrm{~cm} / \mathrm{s}$ have been reported for these materials (Stewart 1978; HickTe 1976). To prevent seepage through the joints of adjacent lifts, hydraulic concretes are laid in two lifts and joints are staggered from 2 to 6 feet. These materials passed a 1-year exposure test to landfill leachate conducted by Haxo and White (1976a). Although the installed cost may be higher than for asphaltic membranes, hydraulic concrete appeared to warrant further testing.

\section{Asphalt/Rubber Admixture}

An asphalt/rubber admixture is a newly developed type of liner containing asphalt and graded rubber particles (from discarded tires) mixed in proportions of $75 \%$ aspha1t and $25 \%$ rubber. The advantages of this material include the effects of rubber on asphalt discussed above; however, it differs from rubberized asphalt in rubber concentrations. The rubber acts as an elastomeric aggregate and allows the membrane to deform more than rock aggregate concretes. Volume changes during freeze/thaw of the liner due to 
water absorbed by the rock aggregate are elininated. Frobel et a1. (1977) used this material at an application rate of $4.5 \mathrm{~L} / \mathrm{m}^{2}$ and reported satisfactory results. Permeability is $10^{-7} \mathrm{~cm} / \mathrm{s}$ al though experimental error is suspected and permeability may be lower. Because of the marked difference between the asphalt/rubber admixture and other liners selected for further testing, it was chosen for laboratory screening instead of the rubberized asphalt membrane.

\section{Soil Asphalt}

Soil asphalts consist of local sands and asphalt mixed in proportions of $94 \%$ sand and $6 \%$ asphatt. These materials are not acceptable for liner applications due to high permeability--10 $0^{-3} \mathrm{~cm} / \mathrm{s}$ (Haxo 1976a). This high permeability is caused by the similar sizes of the aggregate material, which complicates compaction and leaves a significant void content. Haxo and white (1976a) reported failure of a soil asphalt membrane exposed to landfill leachate for 1 year.

\section{CONCRETES}

Portland cement-based concretes have been used for many years to line various water impoundment facilities, but these admixtures exhibit several limitations with respect to mill tailings. For example, they are inherently susceptible to spall damage from exposure to freeze/thaw cycling, cracking due to volume changes experienced under repeated wetting and drying, and corrosion from the $\mathrm{SO}_{4}^{-2}$ ions present in several of the tailings leachates. They would require reinforcement if installed where significant substrate subsidence is expected. Water permeability of these concretes is on the order of $10^{-4} \mathrm{~cm} / \mathrm{s}$.

For tailings impoundments where $\mathrm{SO}_{4}^{-2}$ ions are not present, little ground motion is expected, annual precipitation is small, and subgrade water content is nearly constant, concretes that have been produced for use on bridge decks could be considered as Tiner materials. These concretes were developed to minimize damage from freeze/thaw cycling and to protect the steet reinforcement structure from corrosion by de-icing chenicals. 
The se specialized concretes are termed internally sealed concrete, polynerimpregnated concrete, polymer-portland cement concrete, and polymer concrete. However, because these materials have not been developed for Targescale use in ponds, and because of the strength and cracking limitations described below, none could be considered for laboratory testing.

\section{Internally Sealed Concrete}

Internally sealed concrete (ISC) is an admixture of portland cement, aggregate, and wax spheres. After the concrete is cast and partialiy cured, heat is applied to the top surface, melting the wax. Ideally, this seals the inherent interconnected pores in the paving. Typical specifications for these admixtures are presented in Table 4.

TABLE 4. Internally Sealed Concrete Specification

\begin{tabular}{lcc}
\multicolumn{1}{c}{ Item } & & Specification \\
Admixture Density & & $446 \mathrm{~kg} / \mathrm{m}^{3}$ \\
Aggregate Content & & $60 \mathrm{Volume} \%$ \\
Wax Content & 7.8 Volume $\%$ \\
Entrained Air & $4-6 \mathrm{Volume} \%$ \\
Water-Cement Ratio & 0.47 \\
Wax Specifications & \\
$\quad$ Density-- & $60 \mathrm{~kg} / \mathrm{m}^{3}$ \\
Sphere Size-- & $-20+80 \mathrm{Mesh}^{\circ}$ \\
Wax-- & $75 \% \mathrm{Parrafin}$ \\
& $25 \%$ Mortan
\end{tabular}

During curing, electrical heaters are placed on the surface of the concrete to melt the wax. To achieve the best results, the recommended minimum temperature in the concrete during the heat treatment is $>85^{\circ} \mathrm{C}$.

Among the concerns about this concrete sealing mechanism are stresses induced by the thermal gradients present in the concrete and by the thermal 
expansion differences between the cement paste and the aggregate. Limestone aggregate is recommended to minimize the latter stresses; however, the acidic nature of the tajlings leachate would dictate selection of an alternative material. These concretes demonstrate about $25 \%$ reduced compressive strength (Tyson 1978).

\section{Polymer Impregnated Concrete}

Polymer impregnated concrete (PIC) materials are designed to protect the portland cement from chemical attack, reduce freeze/thaw failure, and increase the mechanical properties of the concrete. As with ISC, this is accomplished by filling the pores--in this case with a polymer--as follows:

1. cast the concrete

2. dry the concrete to reduce water in the concrete pores

3. saturate the concrete with a monomer

4. polymerize the monomer.

Typically, liquid monomers are used but gaseous monomers are possible. All cements, aggregates, and admixes are compatible with this technique.

The strength and durability of this compound is clearly a function of the percentage of concrete voids filled with the polymer or, rephrased, a function of the amount of water removed from the cast concrete. Because water removal requires that the concrete be heated, the process is complicated for large applications.

Three methods of polymerizing the monomer are suggested: thermalcatalytic polymerization, promoted-catalytic polymerization, and exposure to gamma radiation from a ${ }^{60}$ co source. The first method requires that heat be applied to the saturated concrete, actuating the catalyst compound to polymerize the monomers. The promoted-catalytic method is similar but occurs at ambient temperature. Limitations of this concrete compound include:

saturating the sloped concrete sections with the low-viscosity monomer solution 
- inhomogeneities in the polymer coating

- stresses induced in polymers caused by the inherent volume reduction during polymerization

- cracks forming in the concreted after the treatment that would expose untreated concrete to the tailings leachate.

$10^{-10} \mathrm{~cm} / \mathrm{s}$ (Hoff 1977 ).

\section{Polymer-Portland Cement Concrete}

Polymer-portiand cement concrete (PPCC) compounds are designed to improve the same characteristics of portland concretes as PIC as well as concrete shear bond, bond-to-aggregate, tensile strength, and flexural strength. Unlike PIC, the polymeric materials are included in the cement/ aggregate admixture prior to casting. Two types of PPCC were considered: those using premix and postmix polymers.

Premix PPCC is the most common form. The monomers are polymerized prior to addition to the concrete/aggregate admixture; and the polymers are generally added as latexes or polymer dispersions and result in distinct polymeric phases in the concrete formed. These compounds are moist-cured for one to three days and allowed to dry under ambient conditions.

Because the polymers are usually added as water-soluble compounds during mixing, prolonged contact with water leads to their dispersion. This eventually would result in a porous concrete with properties similar to ordinary portland cement concrete.

Limited success has been achieved with postmix PPCC. Problems include interference with portland cement hydration, chemical reactions between the cement and monomer, difficulty in producing a homogeneous monomer dispersion, poor bonds between the polymer and aggregate, and achievement of complete polymerization (Hoff et al. 1977). 
Polymer Concrete

Polymer concrete (PC) was the most promising of the nonasphaltic concretes considered. This naterial is a polymer-aggregate admixture and uses the same mixing and installation equipment as portland cement concrete. $P C$ exhibits much greater chemical stability, compressive and tensile strengths and freeze/thaw resistance than portland cement concretes and should have very low permeabilities (Hickey 1969; Hoff et al. 1977).

These concretes are made with a variety of resins or monomers and can be adapted to the required application. Aggregates should be dried to less than 2\% moisture for best service. $P C$ contains between 5 and 30 wt\% monomer. The catalyst-promoter polymerization technique described in the PIC section is recommended (Hoff 1977).

\section{PRESSURE GROUTING FOR LINER FORMATION}

The possibility of creating an effective candidate liner for uraniummill tailings leachate by injecting portland cement or expoxy-based grouts into the subgrade has been evaluated by Tamura and Boegley (1983) for the UMTRA Program and thus was not included in the PNL Tiner program.

\section{GEONEMIRANES}

Polymeric materials in the form of geomembrane liners were also considered. These flexible lining materials can be classified generally as elastomers (rubbers) or plastics. Major advantages include low permeability and the ability to conform to changing substrate conditions caused by freeze/thaw or wet/dry cycling, sink-hole formation, subsidence, etc. Liners are delivered in large sheets, usually containing several factory seams, and are available in a variety of thicknesses and colors, with or wi thout fiber reinforcement (scrim), and in special compositions modifying the basic polymer to meet specific exposure conditions (Stewart 1978). 
These materials require particular care in relation to site selection, ground preparation, and installation. To maximize the potential effectiveness of the liner, potential liner-compromising mechanisms must be minimized. These liners can be damaged by animals or microbes; operation of heavy equipment; poor seaming techniques; stresses that exceed the tensile capabilities of the material during installation or subsequent ground subsidence; perforation by growing plants or sharp objects beneath the liner; potential buildup of gas pressure under the liner for various reasons; and excessive reverse hydrostatic pressure caused by a high water table (Willians 1978; Stewart 1978; E. I. du Pont A; E. I. du Pont C).

Proper design of the tailing impoundment can reduce these potential failure modes. A $15-$ to $30 \mathrm{~cm}$ layer of sandy soil or tailings placed over the liner will protect it from damage by equipment or animals and would be required for ozone or ultraviolet-sensitive polymers. Biocides or fungicides can be incorporated into the liner during initial processing to protect it from microbial attack, and no liner polymer that would nourish microbes should be selected. Seams constructed in the field are less reliable than those done by the supplier and should be kept to a minimum; however, the selected liner should demonstrate good field-seaming characteristics and each joint should be checked by conventional techniques.

The liner can be protected from overstressing by a number of techniques. The ground beneath the liner can be compacted to prevent sink-hole formation; installation crews can ensure that the liner is not stretched during installation; liners can be purchased with internal fabric reinforcement (scrim); and the slope of impoundment walls can be limited to $1 \mathrm{~m}$ vertical and $2 \mathrm{~m}$ horizontal. It is also recommended that any wall seams be oriented so that they run vertically (Schultz and Miklas 1980).

In addition to precompaction, several other preparations are necessary prior to liner installation. The ground must be sterilized to prevent vegetation from penetrating the liner, and all organic materials must be removed to prevent gas-bubble formation beneath the liner. In areas where high winds or widely fluctuating water tables are expected, vents and 
sloped pond bottoms as well as scrims are recommended to prevent damage from gas accumulation (Lubina, Hovater and McCready 1979; E. I, du Pont C). Scrins are also recommended when high water tables can produce excessive hydrostatic pressures beneath the liner.

It is generally thought, because of limited historical use data in comparison with asphalts and clays, that polymeric geomembranes could not be considered a long-term (1000-year) barrier for uranium mill tailings. As a basis for comparison, however, one of the more promising materials discussed below was selected for laboratory testing.

\section{Elastomers}

\section{Butyl Rubber}

Butyl rubber is a polymer containing $97 \%$ isobutylene and $3 \%$ isoprene. This material has demonstrated lifetimes of greater than 20 years lining potable water reservoirs (Stewart 1978). Butyl rubber has good weather resistance, is serviceable between -7 and $120^{\circ} \mathrm{C}$, will elongate greater than $300 \%$ and recover in this temperature range, has low water absorption and permeability $\left[1.1 \times 10^{-11} \mathrm{~cm} / \mathrm{s}\right.$ (Haxo 1976a)], and is available in $0.5-$ to 3.2-mm (20- to 125-mi1) thicknesses with or without scrim (Stewart 1978; Villiams 1978; Haxo 1976a).

Haxo et a1. (1979) demonstrated that the properties of butyl changed little when the material was exposed to landfill leachate for 8 months (Table 5); and the results of preliminary tests with a $\mathrm{HNO}_{3}$ solution ( 1.5 pH) are shown in Table 6 (Haxo et a 1. 1977; Haxo et a 1. 1979; Haxo 1976b). However, the material has low resistance to hydrocarbons; requires a difficult, two-part seaming procedure that is highly weather-dependent; and is less resistant to embrittlement by radiation damage than other elastomers considered. The pertinent characteristics of butyl rubber are presented in Table 7. 0 ther elastomeric materials have improved characteristics when exposed to conditions expected in a uranium-mill tailings repository. 
TABLE 5. Summary of the Effects of Immersion of Polymeric Membrane Liners in Leachate for Eight Months

\begin{tabular}{|c|c|c|c|c|c|c|}
\hline \multirow[b]{2}{*}{ Polymer } & \multirow{2}{*}{$\begin{array}{l}\text { Number } \\
\text { of Liners } \\
\text { in Test }\end{array}$} & \multirow[b]{2}{*}{$\begin{array}{l}\text { Absorption of } \\
\text { Leachate, } \%\end{array}$} & \multicolumn{3}{|c|}{$\begin{array}{c}\% \text { Original Value } \\
\text { for Unexposed Membrane }\end{array}$} & \multirow{2}{*}{$\begin{array}{c}\text { Change in } \\
\text { Hardness, } \\
\text { Points } \\
\end{array}$} \\
\hline & & & $\begin{array}{l}\text { Tensile } \\
\text { Strength }\end{array}$ & Elongation & $S-200$ & \\
\hline Buty1 Rubber & 1 & $1-2$ & $90-97$ & $104-106$ & $85-86$ & 0 \\
\hline Chlorinated Polyethylene & 3 & $8-10$ & $80-115$ & $64-135$ & $84-123$ & -5 to -1 \\
\hline Chlorosulfonated Polyethylene & 3 & $13-19$ & $82-124$ & $97-107$ & $47-113$ & -20 to -4 \\
\hline Elasticized Polyolefin & 1 & 0.1 & $86-94$ & $91-92$ & $102-106$ & 0 \\
\hline Ethylene Propylene Rubber & 5 & $1-13.5$ & $90-91$ & $76-138$ & $98-220$ & -1 to +2 \\
\hline Neoprene & 4 & $1-19$ & $69-100^{(a)}$ & $82-103^{(a)}$ & $79-102^{(a)}$ & -11 to +5 \\
\hline Polybutylene & 1 & 0.1 & $96-99$ & $96-97$ & $99-103$ & -3 \\
\hline Polyester Elastomer & 1 & 2.0 & $99-115$ & $101-108$ & $95-110$ & -4 \\
\hline Polyethylene & 1 & 0.6 & $110-180$ & $96-181$ & $100-116$ & -7 \\
\hline Polyvinyl Ch1oride & 7 & $1-3$ & $91-110$ & $98-129$ & $76-102$ & -2 to +1 \\
\hline Polyvinyl Chloride plus Pitch & 1 & 6.0 & 92 & $109-133$ & $93-108^{(b)}$ & -2 \\
\hline
\end{tabular}

(a) Data on fabric-reinforced neoprene liner number 42 were not included.

(b) S-100 - unexposed specimen broke at $150 \%$ elongation. 
TABLE 6. Effect of Immersing Liner and Sealing Materials in Hazardous Wastes- A Preliminary

(Unit: $\therefore$ Increase in Area of Portion of 5trip Immersed)

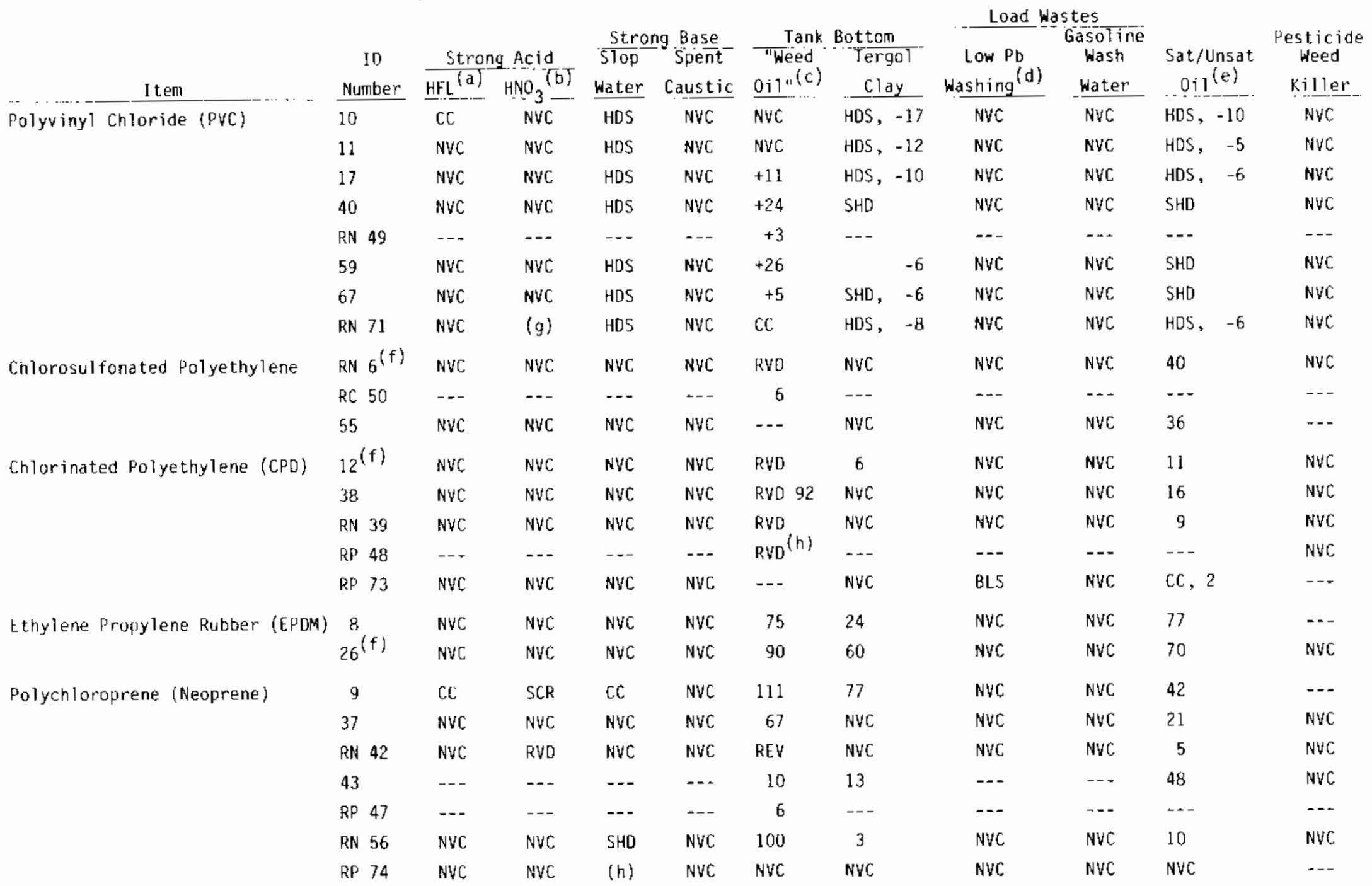




\section{TABLE 6. Continued}

(Unit: $\stackrel{4}{*}$ Increase in Area of Portion of Strip Immersed)

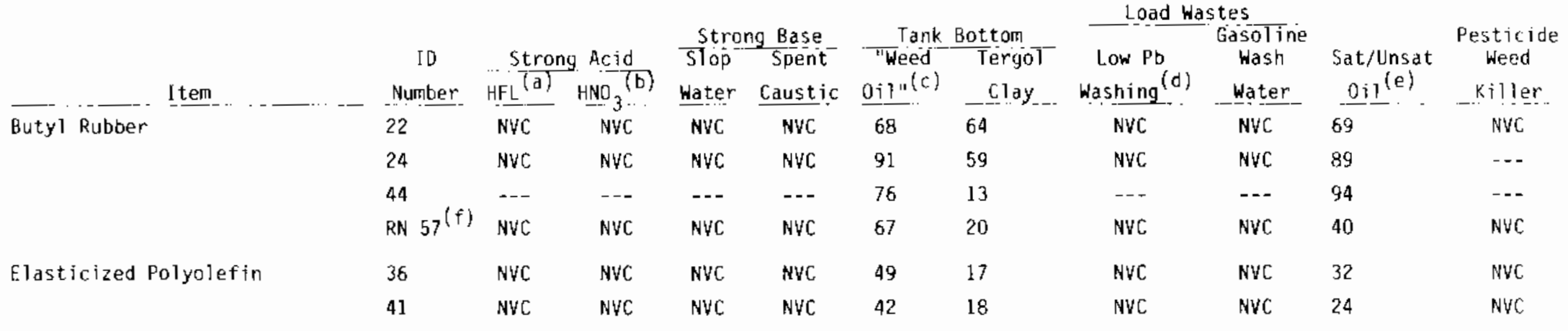

(a) Hydrofluoric

(b) Hydrofluoric, acetic, and nitric acids.

$\sim$ (c) "Weed oil" (highly aromatic) 30:; water $70 \%$ (tank botton waste)

(d) Low-lead gas washings.

(e) 0 il pond 104 .

(f) Materials incorporated in cells.

(g) $0.10 \mathrm{in}$. swell in each direction, curled, and delaminated.

(b) Complete loss of tear strength.

(i) Neoprene in satisfactory condition but reinforcing fabric (polyester) dissolved.

(j) Black rubs off, indicating possible dissolving of the liner.

ABEREVIATIONS (cont'd).

HDS - Hardened and shrank

NVC - No visible change

RC - Reinforced and cured

REV - Reinforced

$R F$ - Reinforced with fabric

RN - Reinforced with nylon

RP - Reinforced with polyester

RVD - Removed specimen from waste because of

excessive swelling or disintegration

ABEREVIATIONS:

ABS - Absorbed waste

BLS - Blistering of surface

CC - Color change

DEL - Delaminated

DIS - Dissolved or disintegrated

SCR - Surface cracking and hardening

SFT-1 - Softened above waste

SFT-2 - Softened in waste

SHO - Slightly hardened

SHR - Shrank

5S - Slight Ty swollen 


\section{TABLE 7. Properties of Polymeric Liner Membranes Installed as Barriers}

\begin{tabular}{|c|c|c|c|c|c|c|c|c|c|c|c|c|}
\hline $\begin{array}{l}\text { Celi Number } \\
\text { Liner Number } \\
\text { Material }\end{array}$ & \multicolumn{2}{|c|}{$\begin{array}{c}1,19 \\
21\end{array}$} & \multicolumn{2}{|c|}{$\begin{array}{c}2,20 \\
17 \\
\text { Polyvinyl } \\
\text { Chloride }\end{array}$} & \multicolumn{2}{|c|}{ Butyl Rubber } & \multicolumn{2}{|c|}{$\begin{array}{c}4,22 \\
6 \\
\text { Hypalon, with } \\
\text { Nylon Scrim! }\end{array}$} & \multicolumn{2}{|c|}{$\begin{array}{c}5,23 \\
16 \\
\text { Ethylene-Propylene- } \\
\text { Diene (EPDM) Rubber }\end{array}$} & \multicolumn{2}{|c|}{$\begin{array}{c}6,24 \\
12 \\
\text { Chlorinated } \\
\text { Polyethylene }\end{array}$} \\
\hline Thickness, mm (0.0001 in.) & \multicolumn{2}{|c|}{$0.25-0.30(10-12)$} & \multicolumn{2}{|c|}{$0.51-0.53(20-21)$} & \multicolumn{2}{|c|}{$1.55-1.65(61-65)$} & \multicolumn{2}{|c|}{$0.81-0.91(32-36)$} & \multicolumn{2}{|c|}{$1.24-1.35(49-53)$} & \multicolumn{2}{|c|}{$0.79-0.81(31-32)$} \\
\hline $\begin{array}{l}\text { Coefficient of water Per- } \\
\text { meability, cm/s }\end{array}$ & & & \multicolumn{2}{|c|}{$7.3 \times 10^{-13}$} & \multicolumn{2}{|c|}{$1.1 \times 10^{-11}$} & \multicolumn{2}{|c|}{$3.6 \times 10^{-12}$} & \multicolumn{2}{|c|}{$2.3 \times 10^{-11}$} & \multicolumn{2}{|c|}{$2.0 \times 10^{-12}$} \\
\hline $\begin{array}{l}\text { Water Absorption, } \\
2 \text { h d } 100^{\circ} \mathrm{C} \\
7 \text { d } 025^{\circ} \mathrm{C} \\
70 \text { d } 025^{\circ} \mathrm{C}\end{array}$ & \multicolumn{2}{|l|}{$\begin{array}{r}-0.61 \\
0.38\end{array}$} & \multicolumn{2}{|l|}{$\begin{array}{l}2.15 \\
0.95\end{array}$} & \multicolumn{2}{|l|}{$\begin{array}{l}0.17 \\
0.18 \\
0.52\end{array}$} & \multicolumn{2}{|l|}{$\begin{array}{l}7.17 \\
2.04 \\
4.52\end{array}$} & \multicolumn{2}{|l|}{$\begin{array}{l}0.47 \\
0.61 \\
1.90\end{array}$} & \multicolumn{2}{|l|}{$\begin{array}{l}2.93 \\
1.43 \\
5.31\end{array}$} \\
\hline $\begin{array}{l}\text { Puncture Test, }{ }^{(\mathrm{a})} 25 \mathrm{~mm} / \mathrm{min} \text {, } \\
\text { maximum force, } \mathrm{N}(\mathrm{ib}) \\
\text { elongation, mm (in.) }\end{array}$ & & & & & \multicolumn{2}{|c|}{$\begin{array}{r}149 \\
29\end{array}\left(\begin{array}{l}33.5) \\
(1.14)\end{array}\right.$} & \multicolumn{2}{|c|}{$\begin{array}{r}131(29.5) \\
26(1.01)\end{array}$} & \multicolumn{2}{|c|}{$\begin{array}{r}141(31.6) \\
35(1.38)\end{array}$} & \multicolumn{2}{|c|}{$\begin{array}{r}150(33.8) \\
26(1.03)\end{array}$} \\
\hline $\begin{array}{l}\text { Puncture rest, } 500 \mathrm{~mm} / \mathrm{min} \text {, } \\
\text { maximum force, } \mathrm{N} \text { (lo) } \\
\text { elongation, mm (in.) }\end{array}$ & \multicolumn{2}{|c|}{$\begin{array}{l}61.9(13.9) \\
19(0.76)\end{array}$} & \multicolumn{2}{|c|}{$\begin{array}{r}115(25.8) \\
18(0.69)\end{array}$} & \multicolumn{2}{|c|}{$\begin{array}{r}199(44.8) \\
31(1.22)\end{array}$} & \multicolumn{2}{|c|}{$\begin{array}{r}146(32.9) \\
15(0.60)\end{array}$} & \multicolumn{2}{|c|}{$\begin{array}{r}175(39.4) \\
37(1.44)\end{array}$} & \multicolumn{2}{|c|}{$\begin{array}{r}209(47.0) \\
26(1.04)\end{array}$} \\
\hline $\begin{array}{l}\text { Splice strength, } \\
\text { peel, kN/m (lb/in.) } \\
\text { shear, kN/m (lb/in.) }\end{array}$ & \multicolumn{2}{|c|}{$\begin{array}{l}2.73(15.6) \\
3.54(20.2)\end{array}$} & \multicolumn{2}{|c|}{$\begin{array}{ll}0.70 & (4.0) \\
6.51 & (37.2)\end{array}$} & \multicolumn{2}{|c|}{$\begin{array}{l}0.66(3.8) \\
5.25(30)\end{array}$} & \multicolumn{2}{|c|}{$\begin{array}{l}5.25(30) \\
8.75(50)\end{array}$} & $\begin{array}{l}0.44 \ll \\
2.56 \% 1\end{array}$ & $\begin{array}{l}\text { (5) } \\
4.6)\end{array}$ & $\begin{array}{l}1.75(10 \\
9.99(57\end{array}$ & \\
\hline $\begin{array}{l}\text { Hardness, Shore A } \\
\text { instantaneous } \\
\text { los }\end{array}$ & $\begin{array}{l}98 \\
98\end{array}$ & & $\begin{array}{l}81 \\
76\end{array}$ & & $\begin{array}{l}55 \\
51\end{array}$ & & $\begin{array}{l}81 \\
79\end{array}$ & & $\begin{array}{l}57 \\
54\end{array}$ & & $\begin{array}{l}85 \\
87\end{array}$ & \\
\hline Grain Direction & With & Cross & With & Cross & With & Cross & With & Cross & With & Cross & With & cross \\
\hline 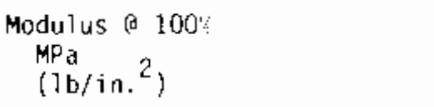 & $\begin{array}{c}8.7 \epsilon_{1} \\
(1270)\end{array}$ & $\begin{array}{l}7.10 \\
(1030)\end{array}$ & $\begin{array}{c}8.69 \\
(1260)\end{array}$ & $\begin{array}{l}7.79 \\
(1130)\end{array}$ & $\begin{array}{r}2.41 \\
(350)\end{array}$ & $\begin{array}{r}2.02 \\
(290)\end{array}$ & $\begin{array}{c}6.90 \\
(1000)\end{array}$ & $\begin{array}{r}5.93 \\
(860)\end{array}$ & $\begin{array}{r}2.41 \\
(350)\end{array}$ & $\begin{array}{r}2,41 \\
(350)\end{array}$ & $\begin{array}{l}8.41 \\
(1220)\end{array}$ & $\begin{array}{r}3.59 \\
(520)\end{array}$ \\
\hline
\end{tabular}




\section{TABLE 7. Continued}

Cell Number

Liner Number

Material

Modulus a 200":

$$
\text { (1b/in. 2) }
$$

Modulus a $300 \%$,

$$
\text { (Ib/in. } \left.{ }^{2}\right)
$$

Tensile Strength,

$$
\text { MPa } 1 \text { in in. }{ }^{2} \text { ) }
$$

Elongation,

$$
\text { set, }
$$

Tear Resistance,$$
\mathrm{kN} / \mathrm{m}
$$

(li / in.) (c)

Lodd $N$

(1b)

- hours to failure

elongation,

\begin{tabular}{|c|c|c|c|c|c|c|c|c|c|c|c|}
\hline Polye & hylene. & & $\begin{array}{l}20 \\
7 \\
\text { vinvyl } \\
\text { ride }\end{array}$ & Buty & Rubber & $\begin{array}{r}4 \\
\text { Mypal } \\
\ldots \text { Nylo }\end{array}$ & $\begin{array}{l}22 \\
5 \\
\text { Acrim } \\
\text { Scrim }\end{array}$ & $\begin{array}{l}\text { Ethylen } \\
\text { Diene }\end{array}$ & $\begin{array}{l}23 \\
\text { if } \\
\text { Propylene- } \\
\text { Pom Rubber }\end{array}$ & $\begin{array}{l}\text { Chlo } \\
\text { Polyt }\end{array}$ & $\begin{array}{l}24 \\
2 \\
\text { inated } \\
\text { hylene }\end{array}$ \\
\hline$\left\{\begin{array}{l}10.1 \\
(1470)\end{array}\right.$ & $\begin{array}{c}7.24 \\
(1050)\end{array}$ & $\begin{array}{c}14.3 \\
(2080)\end{array}$ & $\begin{array}{c}12.8 \\
(1850)\end{array}$ & $\begin{array}{r}5.31 \\
(770)\end{array}$ & $\begin{array}{r}4.21 \\
(610)\end{array}$ & $\begin{array}{c}11.8 \\
(1710)\end{array}$ & $\begin{array}{c}9.17 \\
(1330)\end{array}$ & $\begin{array}{r}5.24 \\
(760)\end{array}$ & $\begin{array}{r}5.24 \\
(760)\end{array}$ & $\begin{array}{l}12.5 \\
\{1820\}\end{array}$ & $\begin{array}{r}5.79 \\
\{840\}\end{array}$ \\
\hline $\begin{array}{l}11.6 \\
(1680)\end{array}$ & $\begin{array}{l}7.72 \\
(1120)\end{array}$ & & & $\begin{array}{c}8.48 \\
(1230)\end{array}$ & $\begin{array}{c}6.90 \\
(1000)\end{array}$ & & & $\begin{array}{c}7.72 \\
(1120)\end{array}$ & $\begin{array}{c}7.72 \\
(1120)\end{array}$ & $\begin{array}{c}17.0 \\
(2460)\end{array}$ & $\begin{array}{c}8.27 \\
(1200)\end{array}$ \\
\hline $\begin{array}{l}11.7 \\
(1700)\end{array}$ & $\begin{array}{l}17.9 \\
(2590)\end{array}$ & $\begin{array}{c}18.2 \\
(2640)\end{array}$ & $\begin{array}{c}17.4 \\
(2520)\end{array}$ & $\begin{array}{c}9.93 \\
(1440)\end{array}$ & $\begin{array}{c}9.86 \\
(1430)\end{array}$ & $\begin{array}{c}13.2 \\
(1920)\end{array}$ & $\begin{array}{l}11.1 \\
(1610)\end{array}$ & $\begin{array}{l}10.4 \\
(1510)\end{array}$ & $\begin{array}{c}9.93 \\
(1440)\end{array}$ & $\begin{array}{c}17.0 \\
(2460)\end{array}$ & $\begin{array}{l}14.3 \\
(2080)\end{array}$ \\
\hline $\begin{array}{l}320 \\
177\end{array}$ & $\begin{array}{l}690 \\
667\end{array}$ & $\begin{array}{r}270 \\
68\end{array}$ & $\begin{array}{r}290 \\
77\end{array}$ & $\begin{array}{r}360 \\
15\end{array}$ & $\begin{array}{r}430 \\
18\end{array}$ & $\begin{array}{l}250 \\
115\end{array}$ & $\begin{array}{l}250 \\
106\end{array}$ & $\begin{array}{r}420 \\
13\end{array}$ & $\begin{array}{r}400 \\
19\end{array}$ & $\begin{array}{l}300 \\
199\end{array}$ & $\begin{array}{l}520 \\
230\end{array}$ \\
\hline $\begin{array}{r}72.6 \\
(415)\end{array}$ & $\begin{array}{r}63.0 \\
\{360\}\end{array}$ & $\begin{array}{r}61.6 \\
(352)\end{array}$ & $\begin{array}{r}55.5 \\
(317)\end{array}$ & $\begin{array}{r}31.5 \\
(180)\end{array}$ & $\begin{array}{r}31.5 \\
(180)\end{array}$ & $\begin{array}{r}56.0 \\
(320)\end{array}$ & $\begin{array}{r}49.0 \\
(280)\end{array}$ & $\begin{array}{l}31.7 \\
(181)\end{array}$ & $\begin{array}{r}31.7 \\
(181)\end{array}$ & $\begin{array}{r}47.2 \\
(270)\end{array}$ & $\begin{array}{r}42.0 \\
(240)\end{array}$ \\
\hline $\begin{array}{r}7.78 \\
(1.75)\end{array}$ & $\begin{array}{r}7.78 \\
(1.75)\end{array}$ & $\begin{array}{l}14.0 \\
(3.15)\end{array}$ & $\begin{array}{c}12.7 \\
(2.85)\end{array}$ & $\begin{array}{l}14.0 \\
(3.14)\end{array}$ & $\begin{array}{l}12.5 \\
(2.82)\end{array}$ & $\begin{array}{c}25.3 \\
(5.68)\end{array}$ & $\begin{array}{r}29.8 \\
(6.7)\end{array}$ & $\begin{array}{r}8.18 \\
(1.84)\end{array}$ & $\begin{array}{r}6.89 \\
(1.55)\end{array}$ & $\begin{array}{l}2.22 \\
(5.0)\end{array}$ & $\begin{array}{c}14.1 \\
(3.18)\end{array}$ \\
\hline $\begin{array}{c}72 \mathrm{NF} \\
13.3\end{array}$ & $\begin{array}{l}72 \mathrm{NF} \\
47.6\end{array}$ & $\begin{array}{r}26 \\
194\end{array}$ & $\begin{array}{r}12 \\
138\end{array}$ & $\begin{array}{l}>94 \mathrm{NF} \\
108\end{array}$ & $\begin{array}{l}94 \mathrm{NF} \\
111\end{array}$ & $\begin{array}{l}96 \mathrm{NF} \\
194\end{array}$ & $253^{2.2}$ & $\begin{array}{l}>143 \mathrm{NF} \\
112\end{array}$ & $\begin{array}{c}>143 \mathrm{NF} \\
102\end{array}$ & $\begin{array}{r}31 \\
186\end{array}$ & $\begin{array}{r}47 \\
344\end{array}$ \\
\hline $\begin{array}{l}5.7 \\
9.5 \\
0.10\end{array}$ & $\begin{array}{c}7.7 \\
19 \\
0.52\end{array}$ & $\begin{array}{l}73 \\
3.88\end{array}$ & $\begin{array}{l}51 \\
7.5\end{array}$ & $\begin{array}{c}84 \\
5.4 \\
0.23\end{array}$ & $\begin{array}{c}83 \\
5.2 \\
0.23\end{array}$ & $\begin{array}{l}15 \\
1.87\end{array}$ & $\begin{array}{c}17 \\
100+ \\
99\end{array}$ & $\begin{array}{l}79 \\
5.6 \\
0.18\end{array}$ & $\begin{array}{l}78 \\
4.7 \\
0.14\end{array}$ & $\begin{array}{l}62 \\
4.5\end{array}$ & $\begin{array}{l}129 \\
176 \\
3.6\end{array}$ \\
\hline
\end{tabular}

- initial stretch.

$$
1 \mathrm{~min},
$$

- creep, cm/crn, $100 \mathrm{~h}$

(a) Method 2065, Fed. Test Methods 101

(b) ASTM D624, Die C

(c) ASTM 0674 - dumbbell specimens, restricted portion 51 (In) (2 in.) long by 6.3 nm $(0.25$ in.) wide.

$N F=$ no failure. 
Ethylene Propylene Rubber (Norde?, EPDM)

Ethylene propylene rubber is a diene monomer similar to butyl rubber. Norde 7 is resistant to ozone and UV attack, is servicable between -50 and $100^{\circ} \mathrm{C}$, and is not susceptible to damage from microbial attack. Nordel is available with or without scrim and in 20- to 60-mil thicknesses (E. I. du Pont A, B and C; Williams 1978). Although the durability of Nordel with hydrocarbons is superior to that of butyl rubber, it is not recommended for liner applications (E. I. du Pont A). As with all vulcanized materials, Nordel presents seaming difficulties; and Haxo et al. (1979) reported Nordel to be more affected than butyl rubber by landfill leachate (see Table 5). They also reported a significant loss of transverse flexibility during the exposure period. EPOM is slightly less resistant to radiation damage than hypalon (a) (Mattia and Luh 1971), which is discussed in the following section. Properties of unexposed EPDM are listed in Table 4.

\section{Chlorosulfonated Polyethylene}

Chiorosulfonated polyethylene (CSPE), which is also called hypalon, is obtained by the reaction of polyethylene in solution with chlorine and sulfur dioxide. When delivered to the installation site, hypalon exhibits properties associated with plastics and self-vulcanizes upon exposure to UV radiation and soil moisture. The product has the following characteristics:

- is resistant to both ozone and ultraviolet light (UV)

- is very impermeable to water $\left(3.6 \times 10^{-12} \mathrm{~cm} / \mathrm{s}\right.$ for a 34-mi 1 1 iner)

- performs satisfactorily from -18 to $120^{\circ} \mathrm{C}$

- is readily seamed under wet conditions

- resists microbial attack

(a) Hypalon is a registered trademark of E. I. du Pont de Nemours and Company. 
- is resistant to $\mathrm{H}_{2} \mathrm{SO}_{4}$ oxidizing chemicals (kerosene may be a problen, however)

- is available in $20,000-\mathrm{ft}^{2}$ rolls, 4 to $6 \mathrm{ft}$ wide, with or without scrim (E. I. du Pont B; E. I. du Pont C; Haxo 1976a).

CSPE will burn as long as a heat source is supplied but will selfextinguish if the source is removed. Portions of the liner to be seamed must be shielded from UV radiation, and the material is relatively weak without fabric reinforcement (E. I. du Pont A; E. I. du Pont C; Stewart 1978). A variety of scrims is available, but polypropylene is recommended because its acid resistance is superior to nylon and polyester. Use of polypropolyene scrim, however, requires an opaque hypalon because it is not UV-resistant (Edwald 1973).

of all the elastomers studied, hypalon demonstrated the best resistance to hardening due to exposure to gamma radiation and remained relatively unchanged up to $10^{7} \mathrm{R}$ exposure (E. I. du Pont $\mathrm{D}$ ). Table 8 presents the effects of gamma radiation on several characteristics of the polymers under consideration (Mattia and Luh 1971).

Haxo and White (1976a) determined that exposure to landfill leachate for 1 year causes a hypalon liner to swell and decreases the seam-peel resistance (see Table 9). However, the resulting seam-peel strength was on the order of the other polymers tested, and the hypalon seam-shear strength remained among the highest rated. As expected, the preliminary study (reported in Table 6) showed no damage to a hypalon liner exposed to a low $\mathrm{pH}$ solution (Haxo 1976b).

In addition, hypalon was chosen as part of the seepage barrier installed at the Cotter Uranium Mill for tailings impoundment. Lubina, Hovater and McCready (1979) reported that du Pont predicts that more than 40 years of service could be expected from an exposed hypalon liner, and indefinite life if the liner is protected by an earthen layer. 
TABLE 8. Effect of Gamma Radiation (a) on Physical Properties (Blodgett and Fisher 1969)

\begin{tabular}{|c|c|c|c|c|c|c|c|c|c|c|c|c|}
\hline & PVC & $\begin{array}{l}\text { High } \\
\text { Density } \\
\text { PE }\end{array}$ & $\begin{array}{l}\text { Black } \\
\text { XLPE }\end{array}$ & $\begin{array}{l}\text { Unfilled } \\
\text { XLPE }\end{array}$ & ȘBR & EPDM & EPM & Butyl & Silicone & Neoprene & Hypalon & $\begin{array}{l}\text { Chlorinated } \\
\text { PE }\end{array}$ \\
\hline \multicolumn{13}{|l|}{ Original Properties } \\
\hline $\begin{array}{l}\text { 200: Modulus, psi } \\
\text { Tensile Strength, psi } \\
\text { Elongation at Break, }\end{array}$ & $\begin{array}{r}2415 \\
2601 \\
250\end{array}$ & $\begin{array}{r}1000 \\
2213 \\
640\end{array}$ & $\begin{array}{r}1767 \\
2045 \\
270\end{array}$ & $\begin{array}{r}1260 \\
2272 \\
480\end{array}$ & $\begin{array}{r}580 \\
1520 \\
460\end{array}$ & $\begin{array}{r}1033 \\
1443 \\
470\end{array}$ & $\begin{array}{l}730 \\
872 \\
300\end{array}$ & $\begin{array}{l}520 \\
798 \\
450\end{array}$ & $\begin{array}{r}859 \\
1191 \\
290\end{array}$ & $\begin{array}{r}930 \\
2544 \\
550\end{array}$ & $\begin{array}{r}884 \\
2113 \\
560\end{array}$ & $\begin{array}{r}626 \\
2170 \\
670\end{array}$ \\
\hline \multicolumn{13}{|l|}{$\begin{array}{l}200 \text { Modulus, Retention } \\
\text { After Irradiation, Rads }\end{array}$} \\
\hline $\begin{array}{l}5 \times 10^{5} \\
5 \times 10^{6} \\
5 \times 10^{7} \\
1 \times 10^{8}\end{array}$ & $\begin{array}{r}81 \\
95 \\
+ \\
+\end{array}$ & $\begin{array}{r}95 \\
98 \\
+ \\
+\end{array}$ & $\begin{array}{r}125 \\
115 \\
+ \\
95\end{array}$ & $\begin{array}{r}96 \\
102 \\
108 \\
+\end{array}$ & $\begin{array}{r}106 \\
121 \\
150 \\
+\end{array}$ & $\begin{array}{r}100 \\
94 \\
120 \\
+\end{array}$ & $\begin{array}{r}116 \\
127 \\
+ \\
+\end{array}$ & $\begin{array}{r}103 \\
69 \\
++ \\
++\end{array}$ & $\begin{array}{r}75 \\
112 \\
98 \\
+++\end{array}$ & $\begin{array}{l}107 \\
103 \\
160 \\
-\end{array}$ & $\begin{array}{r}116 \\
156 \\
203 \\
-\end{array}$ & $\begin{array}{r}108 \\
152 \\
+ \\
-\end{array}$ \\
\hline $\begin{array}{l}\text { Tensile Strength, } \\
\text { Nfter Retention }\end{array}$ & & & & & - & & & & & & & \\
\hline $\begin{array}{l}5 \times 10^{5} \\
5 \times 10^{6} \\
5 \times 10^{8} \\
1 \times 10^{8}\end{array}$ & $\begin{array}{r}80 \\
88 \\
61 \\
-\end{array}$ & $\begin{array}{r}96 \\
98 \\
123 \\
118\end{array}$ & $\begin{array}{r}122 \\
112 \\
101 \\
95\end{array}$ & $\begin{array}{r}102 \\
97 \\
70 \\
59\end{array}$ & $\begin{array}{r}98 \\
100 \\
82 \\
40\end{array}$ & $\begin{array}{r}104 \\
97 \\
93 \\
79\end{array}$ & $\begin{array}{r}101 \\
106 \\
119 \\
90\end{array}$ & $\begin{array}{l}96 \\
58 \\
++ \\
++\end{array}$ & $\begin{array}{r}76 \\
100 \\
100 \\
+++\end{array}$ & $\begin{array}{r}104 \\
98 \\
77 \\
-\end{array}$ & $\begin{array}{r}106 \\
113 \\
124 \\
-\end{array}$ & $\begin{array}{r}112 \\
98 \\
135 \\
-\end{array}$ \\
\hline \multicolumn{13}{|l|}{$\begin{array}{l}\text { f. longation, Retention } \\
\text { After Irradiation, Rads }\end{array}$} \\
\hline $\begin{array}{l}5 \times 10^{5} \\
5 \times 10^{6} \\
5 \times 10^{8} \\
1 \times 10^{8}\end{array}$ & $\begin{array}{r}100 \\
80 \\
40 \\
-\end{array}$ & $\begin{array}{r}103 \\
103 \\
- \\
2\end{array}$ & $\begin{array}{r}104 \\
96 \\
48 \\
37\end{array}$ & $\begin{array}{l}90 \\
96 \\
58 \\
25\end{array}$ & $\begin{array}{l}93 \\
96 \\
70 \\
33\end{array}$ & $\begin{array}{r}111 \\
102 \\
47 \\
32\end{array}$ & $\begin{array}{l}96 \\
81 \\
41 \\
26\end{array}$ & $\begin{array}{l}93 \\
87 \\
++ \\
++\end{array}$ & $\begin{array}{r}107 \\
90 \\
34 \\
+++\end{array}$ & $\begin{array}{r}96 \\
93 \\
46 \\
-\end{array}$ & $\begin{array}{l}89 \\
86 \\
59 \\
-\end{array}$ & $\begin{array}{l}99 \\
63 \\
18 \\
-\end{array}$ \\
\hline
\end{tabular}

(a) Radiation Source - For exposures of $5 \times 10^{5}, 5 \times 10^{6}$, and $5 \times 10^{7}$, cobalt-60 source was used (gamma = 1.17 to 1.332 mev and beta $=0.31$ wev, at a dose rate of $\left.5 \times 10^{5} \mathrm{rads} / \mathrm{h}\right)$. For exposure of $1 \times 10^{8}$, tsso Research and Engineering Company's radiation core in air and water was used, with the same dose rate as above.

+ Elongated less than $200 \%$

++ Degraded (low strength due to chain scissions)

++ Brittle

- No value reported 
TABLE 9. Effect on Properties (a) of Polymeric Membrane Liners of One Year of Exposure to Lechate from Simulated Sanitary Landfills (data in U.S. Customary units)

\begin{tabular}{|c|c|c|c|c|c|c|c|}
\hline$\ldots \ldots \ldots$ Iten & $\begin{array}{l}\text { Exposure } \\
\text { Time, } \\
\text { Years }\end{array}$ & Polyethylene & $\begin{array}{l}\text { Polyvinyl } \\
\text { Chloride }\end{array}$ & Buty? & $\begin{array}{l}\text { Chlorosulfonated } \\
\text { Polyethylene. }\end{array}$ & $\begin{array}{l}\text { Ethylene } \\
\text { Propylene } \\
\text { Rubber }\end{array}$ & $\begin{array}{l}\text { Chlorinated } \\
\text { Polyethylene }\end{array}$ \\
\hline Liner Number & --- & 21 & 17 & 7 & 6 & 16 & 12 \\
\hline Generator Number & --- & 19 & 20 & 21 & 22 & 23 & 24 \\
\hline Thickness, mils & $\begin{array}{l}0 \\
1\end{array}$ & $\begin{array}{c}11-12 \\
11\end{array}$ & $\begin{array}{c}20-21 \\
21\end{array}$ & $\begin{array}{c}61-65 \\
64\end{array}$ & $\begin{array}{c}32-36 \\
38\end{array}$ & $\begin{array}{c}49-53 \\
51\end{array}$ & $\begin{array}{c}31-32 \\
35\end{array}$ \\
\hline Tensile Strength, psi & $\begin{array}{l}0 \\
1\end{array}$ & $\begin{array}{l}2145 \\
2465\end{array}$ & $\begin{array}{l}2580 \\
2350\end{array}$ & $\begin{array}{l}1435 \\
1395\end{array}$ & $\begin{array}{l}1765 \\
1640\end{array}$ & $\begin{array}{l}1475 \\
1455\end{array}$ & $\begin{array}{l}2270 \\
1810\end{array}$ \\
\hline Elongation at Break, & $\begin{array}{l}0 \\
1\end{array}$ & $\begin{array}{l}505 \\
560\end{array}$ & $\begin{array}{l}280 \\
330\end{array}$ & $\begin{array}{l}395 \\
410\end{array}$ & $\begin{array}{l}250 \\
300\end{array}$ & $\begin{array}{l}410 \\
435\end{array}$ & $\begin{array}{l}410 \\
400\end{array}$ \\
\hline Tensile Set, : & $\begin{array}{l}0 \\
1\end{array}$ & $\begin{array}{l}422 \\
432\end{array}$ & $\begin{array}{l}73 \\
57\end{array}$ & $\begin{array}{l}17 \\
14\end{array}$ & $\begin{array}{l}111 \\
106\end{array}$ & $\begin{array}{l}16 \\
12\end{array}$ & $\begin{array}{l}429 \\
208\end{array}$ \\
\hline$s-200,{ }^{(a)} p s i$ & $\begin{array}{l}0 \\
1\end{array}$ & $\begin{array}{l}1260 \\
1205\end{array}$ & $\begin{array}{l}1965 \\
1550\end{array}$ & $\begin{array}{l}690 \\
685\end{array}$ & $\begin{array}{l}1520 \\
1245\end{array}$ & $\begin{array}{l}760 \\
740\end{array}$ & $\begin{array}{l}1330 \\
1090\end{array}$ \\
\hline Tear strength (Die C), ppi & $\begin{array}{l}0 \\
1\end{array}$ & $\begin{array}{l}390 \\
496\end{array}$ & $\begin{array}{l}335 \\
450\end{array}$ & $\begin{array}{l}180 \\
202\end{array}$ & $\begin{array}{l}300 \\
305\end{array}$ & $\begin{array}{l}181 \\
195\end{array}$ & $\begin{array}{l}155 \\
320\end{array}$ \\
\hline Hardness (Duro A - $10 \mathrm{~s}$ ) & $\begin{array}{l}0 \\
1\end{array}$ & $\begin{array}{r}98 \\
---\end{array}$ & $\begin{array}{l}76 \\
64\end{array}$ & $\begin{array}{c}51 \\
50.5\end{array}$ & $\begin{array}{l}79 \\
64\end{array}$ & $\begin{array}{l}54 \\
51.5\end{array}$ & $\begin{array}{c}85 \\
65.5\end{array}$ \\
\hline $\begin{array}{l}\text { Puncture Resistance (b) } \\
\text { force, } 1 \mathrm{~b}\end{array}$ & $\begin{array}{l}0 \\
1\end{array}$ & $\begin{array}{l}11.9 \\
14.8\end{array}$ & $\begin{array}{l}25.8 \\
30.1\end{array}$ & $\begin{array}{l}44.8 \\
49.5\end{array}$ & $\begin{array}{l}32.9 \\
57.0\end{array}$ & $\begin{array}{l}39.4 \\
40.1\end{array}$ & $\begin{array}{l}47.0 \\
49.8\end{array}$ \\
\hline ETongation, in. & $\begin{array}{l}0 \\
1\end{array}$ & $\begin{array}{l}0.76 \\
0.80\end{array}$ & $\begin{array}{l}0.69 \\
0.70\end{array}$ & $\begin{array}{l}1.22 \\
1.20\end{array}$ & $\begin{array}{l}0.60 \\
0.88\end{array}$ & $\begin{array}{l}1.44 \\
1.18\end{array}$ & $\begin{array}{l}1.04 \\
0.98\end{array}$ \\
\hline Volatiles at $105^{\circ} \mathrm{C}$ & 1 & 0.02 & 3.55 & 2.02 & 12.76 & 5.54 & 6.84 \\
\hline Seam Strength Peel, ppi & $\begin{array}{l}0 \\
1\end{array}$ & $\begin{array}{l}15.6 \\
10.3^{(c)}\end{array}$ & $\begin{array}{r}40 \\
5.1\end{array}$ & $\begin{array}{l}3.8 \\
2.9\end{array}$ & $\begin{array}{r}30.0 \\
3.4\end{array}$ & $\begin{array}{l}2.5 \\
2.0\end{array}$ & $\begin{array}{r}10.0 \\
5.1\end{array}$ \\
\hline Shear, ppi & $\begin{array}{l}0 \\
1\end{array}$ & $\begin{array}{l}20.2 \\
11.4\end{array}$ & $\begin{array}{l}37.2 \\
25.6\end{array}$ & $\begin{array}{l}30.0 \\
42.0\end{array}$ & $\begin{array}{r}50 \\
40.2\end{array}$ & $\begin{array}{l}14.6 \\
24.3\end{array}$ & $\begin{array}{l}57 \\
35\end{array}$ \\
\hline
\end{tabular}

(a) Stress at 200 elongation.

(b) Rate of penetration of probe $20 \mathrm{in.} / \mathrm{min}$.

(c) Seam in polyethylene liner used in steel column. Tabs in liner specimens mounted in base were too short. 
Because of its advantageous characteristics, chlorosulfonated polyethylene was selected as the geomembrane material for laboratory screening tests. The results of these tests were used as the basis for comparisons with asphart and clay liners.

\section{Neoprene}

Heoprene is a chlorine-containing synthetic rubber designed to resist hydrocarbon solvents. Neoprenes are serviceable between -20 and $90^{\circ} \mathrm{C}$, resist attack by ozone and microbes, and are avajlable in 20- to 60-mil sheets (E. I. du Pont $C$ ). However, neoprene is susceptible to damage from acid and strong salt solutions expected in tailings impoundments and exposure to weathering when in contact with earth; and seaming must be performed above $10^{\circ} \mathrm{C}$ (Williams 1978; E. I. du Pont A). Radiation resistance is comparable to hypalon, but neoprene is still not suitable for this application (E. I. du Pont D).

\section{Plastics}

\section{Elasticized Polyolefin $(3110,3111)$}

Elasticized polyolefin is a thermoplastic material with good aging characteristics and resistance to attack by oily solutions, microbes, and solutions between 2 and $13.5 \mathrm{pH}$. Because the material is not vulcanized, field heat-seaming is possible at rates to $360 \mathrm{~m} / \mathrm{hr}$. The material is extruded rather than calandered and is available in $6-m \times 61-m \times 20-m i 1$ sheets; however, because of the extrusion processing, it is not available with fiber reinforcement, which limits its applicability. Moreover, elasticized polyolefin must not be exposed to hydrocarbons, steep slopes $(>33 \%)$, or impoundments where wet/marshy conditions exist. No radiation resistance data are available; and du Pont does not warranty this material (Williams 1978; E. I. du Pont B; E. I. du Pont C).

\section{Polyethylene}

Since polyethylene materials do not contain a plasticizer, extrusionforming processes are required and sheet thickness is 1 imited to $8 \mathrm{~mm}$. 
Polyethylene has poor resistance to UV exposure, requires an adhesive tapestyle seal that is difficult to make, has limited resistance to punctures, and tends to crack in creases. Advantages such as chemical inertness and radiation resistance do not overcome the limitations of polyethylene (Stewart 1978; Williams 1978; E. I. du Pont 0; Haxo 1976a); however, it is available in high- and low-density specifications.

High-density polyethylene appears to have excellent durability under conditions expected in uranium-mill tailings impoundments. Its lack of plasticizers eliminates concerns about microbial attack, and the material is resistant to degradation from hydrocarbons thought to be contained in tailings piles, high acidity, and UV radiation. Although these characteristics made high-density polyethylene worth considering for laboratory testing, it had limited use in the field at the time the selections were made, and chlorosulfonated polyethylene was chosen instead.

\section{Polyvinyl Chloride}

Polyvinyl chloride (PVC) is a calandered material that is available in a wide variety of thicknesses, colors, and compositions. PVC exhibits good resistance to puncture and microbes, has low permeability $\left(7.3 \times 10^{-13} \mathrm{~cm} / \mathrm{s}\right.$ for a 20-mil sheet), and is chemically stable in the environment expected in the tailings piles. Principal disadvantages include susceptibility to breakdown from exposure to uV radiation below $355 \mathrm{\mu m}$ resulting in cross-bonding and increased stiffness, migration of plasticizers also resulting in increased stiffness, and moderate gamma exposures resulting in a compound that becomes soft and tacky. As with low-density polyethylene, the inherent limitations were serious enough to eliminate PVC from further consideration as a candidate liner material (Savartnick 1969; Stewart 1978; Williams 1978; E. I. du Pont D; Haxo 1976a).

\section{Chlorinated Polye thylene}

Chiorinated polyethylene (CPE) is a calandered thermoplastic available in 20- to 45-mil thicknesses with a polyolefin reinforcing sheet. The material is produced by chlorinating high-density polyethylene and can be seamed by adhesive solvent, solvent welding, or heat-sealing techniques. 
CPE is not susceptible to ozone or UV radiation, resists hydrocarbons, is unaffected by microbes, and has a permeability of $2 \times 10^{-12} \mathrm{~cm} / \mathrm{s}$ for a 30-mil sheet. The material, however, is reported to have limited resistance to acids, requires reinforcement for use on slopes, and is not recommended on slopes of more than $2-m$ rise to $3-m$ horizontal. Susceptibility to acid attack preciuded any further consideration. 


\section{LABORATORY LINER EVALUATIONS}

At the completion of preliminary studies, eight of the more promising liner materials were selected for laboratory screening:

- hydraulic asphalt concrete

- catalytic airblown asphalt membrane

- asphalt/rubber membrane

- chlorosulfonated polyethylene (hypalon)

- natural soil from a typical disposal site

- soil amended with sodium bentonite

- Soil amended with Saline Seal 100

- soil amended with GSR-60.

The clay liner materials listed above (sodium bentonite, Saline Seal 100, GSR 60) afforded a broad evaluation of sodium-bentonite-based liner substances. The eight candidate materials were placed in $0.6-m$ diameter exposure columns and subjected to conditions that accelerated the aging process. The performance of each material was evaluated by comparing changes in permeability with time of exposure. The two most suitable liners were identified for further laboratory evaluation and exposure to real-time conditions in the field.

\section{ACCELERATED TESTING METHODOLOGY}

Since EPA regulations (USEPA 1983) require that mill tailings disposal sites conform to 1000-year criteria, the approach chosen as the best way to determine long-term liner effectiveness was to accelerate the aging mechanisms while maintaining conditions as nearly representative of the field as possible. It was considered equally important to select destructive mechanisms that would be encountered in the field. For example, UV degradation of asphalt was not considered because the liner would be covered throughout $i$ ts useful life. Likewise, freeze/thaw and wet/dry cycles were eliminated from practical consideration because a thick cover of tailings and soil over the liners would eliminate the possibility of such extremes. 
The behavior of clay amendments and asphalt and geomembrane liners are such that different accelerated testing methodologies had to be employed. Asphalt and synthetic materials degrade primarily by oxidation, which causes them to become hard and brittle. The most serious degradation mechanism for clay materials is the ion-exchange reaction between Na present in the clays and $\mathrm{Ca}$, which exists in high concentrations in uranium tailings leachates. This ion-exchange reaction decreases the swelling capabilities of the clay, increasing leachate permeability. Both asphalt and clay materials, however, are subject to the same physical stresses caused by ground subsidence when tailings are introduced to the disposal site. Gamma radiation effects over the 1000-year period were also considered.

\section{Oxidation of Asphalts and Synthetics}

Asphalt aging results in physical hardening and embrittlement of the liner materials; and the primary degradation known to occur due to aging is oxidation. When oxidized, asphalt is converted to longer-chain hydrocarbons through the formation of carbonyls. These longer-chained hydrocarbons are more viscous and brittle; thus, they are more prone to crack under physical stresses that might be experienced at a tailings site over a long period (i.e., 1000 years). It is important, therefore, to quantify the rate of aging reactions and predict the condition of the asphalt liner after the 1000-year period. For these reasons, a methodology was developed to accelerate the reaction rate over a reasonable period of time 3 months).

The objective of accelerated aging was to increase the degradation reaction rates of an asphalt liner under simulated mill tailings impoundment conditions. To accomplish this, certain exposure conditions were selected, based on past studies, to accelerate the reaction mechanisms. The reaction rate was determined and compared to those of less accelerated or normal conditions, and this comparison was used to quantify the expected performance lifetime. 
Van Oort (1956) and Blokker and van Hoorn (1959) conducted studies on increasing the degradation rates of asphalt, and both used the following expression for the reaction rate:

$$
R_{A}=k\left(C_{0_{2}}\right)^{n}
$$

where $R_{A}=$ reaction rate of the component of interest

$k=$ the temperature dependent reaction rate constant

$\mathrm{C}_{\mathrm{O}_{2}}=$ concentration of oxygen

$n=$ the dependency of oxygen on reaction rate (referred to as the order of reaction).

The reaction rate constant is dependent upon temperature according to Arrhenius law (Levenspiel 1972):

$$
k=k_{0} e^{-E_{A} / R T}
$$

where $k=$ reaction rate constant

$$
\begin{aligned}
k_{0} & =\text { a proportionality constant } \\
E_{A} & =\text { activation energy } \\
R & =\text { gas law constant } \\
T & =\text { temperature. }
\end{aligned}
$$

Since the reaction rate constant is temperature-dependent, Van 0ort experimented with temperature to increase the reaction rate. By conducting aging experiments in the absence of light, Van 0ort was able to determine the activation energy $E_{A}$ for asphalt experimentally. By plotting $1 n k$ vs $1 / T$, he derived the activation energy at $24 \mathrm{kcal} / \mathrm{mole}$, which is a normal value for organic chemical reactions. At this activation energy, an increase in temperature of $10^{\circ} \mathrm{C}$ above ambient would be expected to increase the reaction rate by a factor of 4.3 .

Blokker and van Hoorn (1959) expanded on Van Dort's investigation of asphalt aging in the dark. Whereas Van 0ort concluded that the extent of 
aging would be limited by diffusion to $7 \mathrm{\mu m}$, BTokker and van Hoorn demonstrated, and substantiated their demonstration with theoretical interpretations, that hardening can occur as deeply as $3 \mathrm{~mm}$ or more. Blokker and van hoorn also increased oxygen pressure to accelerate the reaction rates. Depending upon the order of reaction in the reaction rate equation, the concentration of oxygen in the atmosphere can affect the reaction rate substantially.

Blokker and van Hoorn's experiments showed that $n$ in the reaction rate equation was 0.6 . This relationship indicates that the concentration of oxygen can be instrumental in affecting the aging rates of asphalt. BTokker and van Hoorn also found that the activation energy (the temperature dependence coefficient of the reaction rate constant) was $12 \mathrm{kcal} / \mathrm{mole}$ rather than $24 \mathrm{kcal} / \mathrm{mole}$ as measured by Van Dort. At this new activation energy, a $10^{\circ} \mathrm{C}$ rise over ambient will approximately double the reaction rate, which is sufficient to make temperature a good acceleration parameter. Based on Blokker and van Hoorn's data, temperature and oxygen partial pressure were selected as aging parameters for the PNL liner evaluation study.

To obtain the equivalent of 1000-year aging in 3 months, it was necessary to accelerate the reaction rate by a factor of 4000 . Because temperature and oxygen alone were not expected to accelerate the aging reactions to the equivalent of 1000 years, another parameter was sought. Since most of the uranium mill tailings leachates have considerable oxidizing agents such as sulfates, it was considered possible that increasing the strength of these agents would increase the rate of chemical degradation.

Basic chemistry shows that the concentration of $H$ ions can increase the oxidizing strength of sulfate; therefore, an increase in the $H$ ion content in simulated leachate from a nominal pH of 2.5 to lower $\mathrm{pH}$ values had the potential of increasing the oxidizing strength of sulfates and other oxidizing agents. Thus, three accelerated aging parameters were identified for the uranium mill tailings liner evaluation: temperature, oxygen partial pressure, and acjdity controlled by $\mathrm{pH}$. 
Ion Exchange in Clay Soils and Bentonites

The swelling property of a clay soil depends upon its ability to hold hydrated $\mathrm{Na}$ ions in its exchange complex; and aside from physical disruptions, the failure of a clay liner will result from loss of its swelling property. This can occur either through a cation-exchange reaction that replaces the $\mathrm{Na}$ in the exchange complex with divalent cations such as $\mathrm{Ca}$, or as a result of the dehydrating effect of a high-salt solution that removes water of hydration from the exchangeable $\mathrm{Na}$. Both effects are controlled by the concentration of salts in the contacting solution.

Concentration affects the exchange reaction because it is a massaction phenomenon. In this testing program, it was necessary to establish conditions under which mass-action would occur over a short period of time and be representative of the results that might be obtained over the long term in an actual impoundment. To accelerate the ion-exchange reaction, a thin clay liner was used and pressurized air was introduced to the exposure columns to apply pressure to the leachate solution above the liner. In this manner, it was possible to measure the relationship between permeability and the number of pore volumes of tailings leachate that passed through the liner.

By equating permeability and number of pore volumes in Darcy's equation for saturated flow (Hillel 1980), exposure time in the laboratory can be related to equivalent time in the field by the relationship

$$
\frac{t_{1}\left(L_{1} / L_{2}\right)^{2}}{t_{2} p_{1} / p_{2}}
$$

$$
\text { where } \begin{aligned}
t & =\text { time } \\
L & =1 \text { iner thickness } \\
p & =\text { hydrostatic pressure } \\
1 & =\text { reference to laboratory measurements } \\
2 & =\text { reference to field measurements. }
\end{aligned}
$$


As the relationship shows, liner aging can be accelerated by exerting an increased hydrostatic pressure over a thin liner relative to that expected in the field.

\section{Physical Stresses}

When tailings are introduced to the disposal site, the soil mantle beneath the liner is expected to subside somewhat, and this subsidence will cause elongation or shifting of the liner along the sloped walls of the disposal site. Figure 6 is a model of a typical disposal site. Using this model, anticipated subsidence was calculated to be $33 \mathrm{~cm}$. Assuming a $3: 1$ excavation slope with a tailings depth of $6 \mathrm{~m}$, the maximum elongation a liner must withstand is $0.035 \mathrm{~cm} / \mathrm{cm}$, or $3.5 \%$; therefore, this value was used in the accelerated tests for clay and asphalt and synthetic liners.

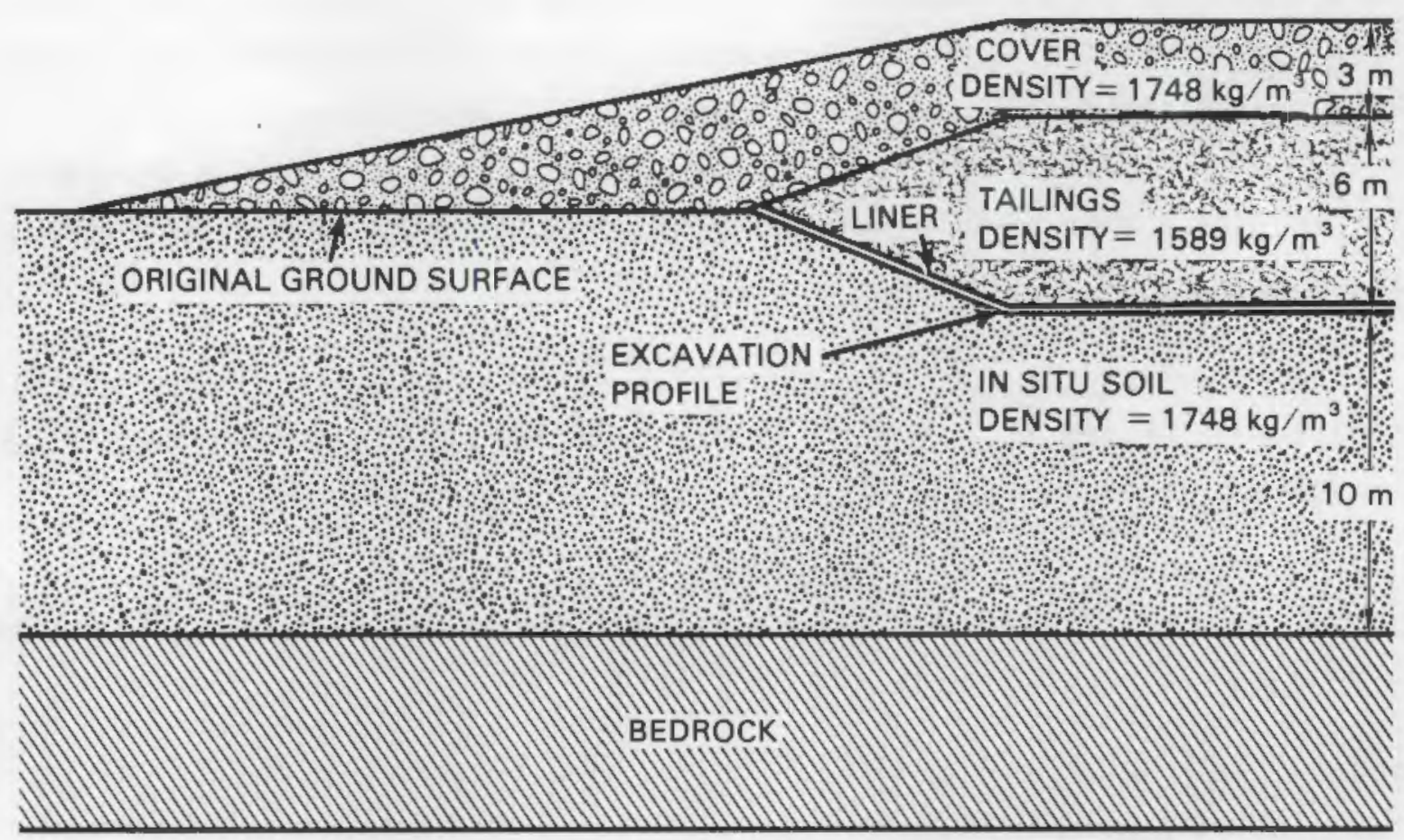

FIGURE 6. Typical Disposal Site for Predicting Subsidence 


\section{Radiation Exposure}

Liner exposure to gamma radiation was estimated for a 1000-year period by making certain assumptions and forming relationships. Assuming the relaxation length of tailings is the same as concrete $(6.5 \mathrm{~cm}$ at $0.35 \mathrm{MeV})$, a surface source was estimated for the tailings above the liner according to the relationship given by Foster and Wright (1973):

$$
S=1 / 2 S v \lambda
$$

where $S=$ surface source, dis/min per $\mathrm{cm}^{2}$

Sv $=$ volume source, dis/min per $\mathrm{cm}^{3}$

$\lambda=$ relaxation length, $\mathrm{cm}$.

The volume source was estimated from the highest gamma activities detected at the Salt Lake City, Shiprock, and Durango tailings piles for the ${ }^{238} \mathrm{U}$ decay chain. The volume source used in the exposure calculation was $36,000 \mathrm{dis} / \mathrm{min}$ per $\mathrm{cm}^{3}\left(16 \mathrm{nCi} / \mathrm{cm}^{3}\right)$, which is much higher than normally found in tailings. The absorbed energy from gamma radiation expected at this level incident to the liners over the 1000 -year period is $10^{4} \mathrm{R}$. A circular sample of each asphalt liner was irradiated from a point source of ${ }^{60} \mathrm{Co}$ at $10^{5} \mathrm{R}$ at the center, and no physical defects were observed on the liner materials.

\section{EXPOSURE COLUMN DESIGN}

Eight sealed columns were fabricated to produce a controlled environment in which the liners were exposed to accelerated aging conditions. The liner exposure columns, shown in Figure 7 , were constructed of $61-\mathrm{cm}$ (24in.) 304L stainless steel pipe and were designed to simulate expected field conditions and to expose the candidate liners to the accelerated aging mechanisms described earlier. Design features are in Figure 8. 
$\stackrel{\leftrightarrow}{\oplus}$

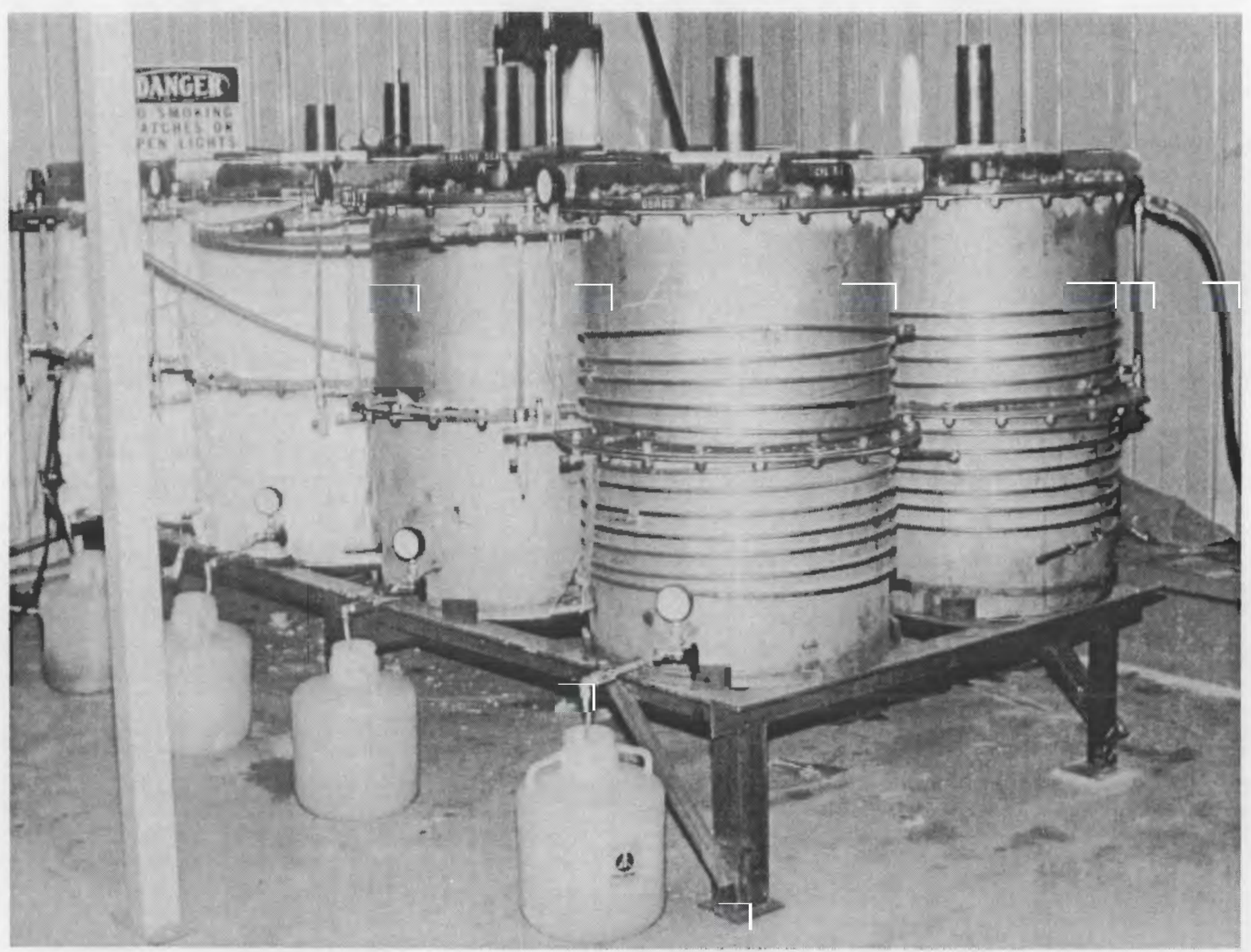

FIGURE 7. Accelerated Aging Laboratory Apparatus 


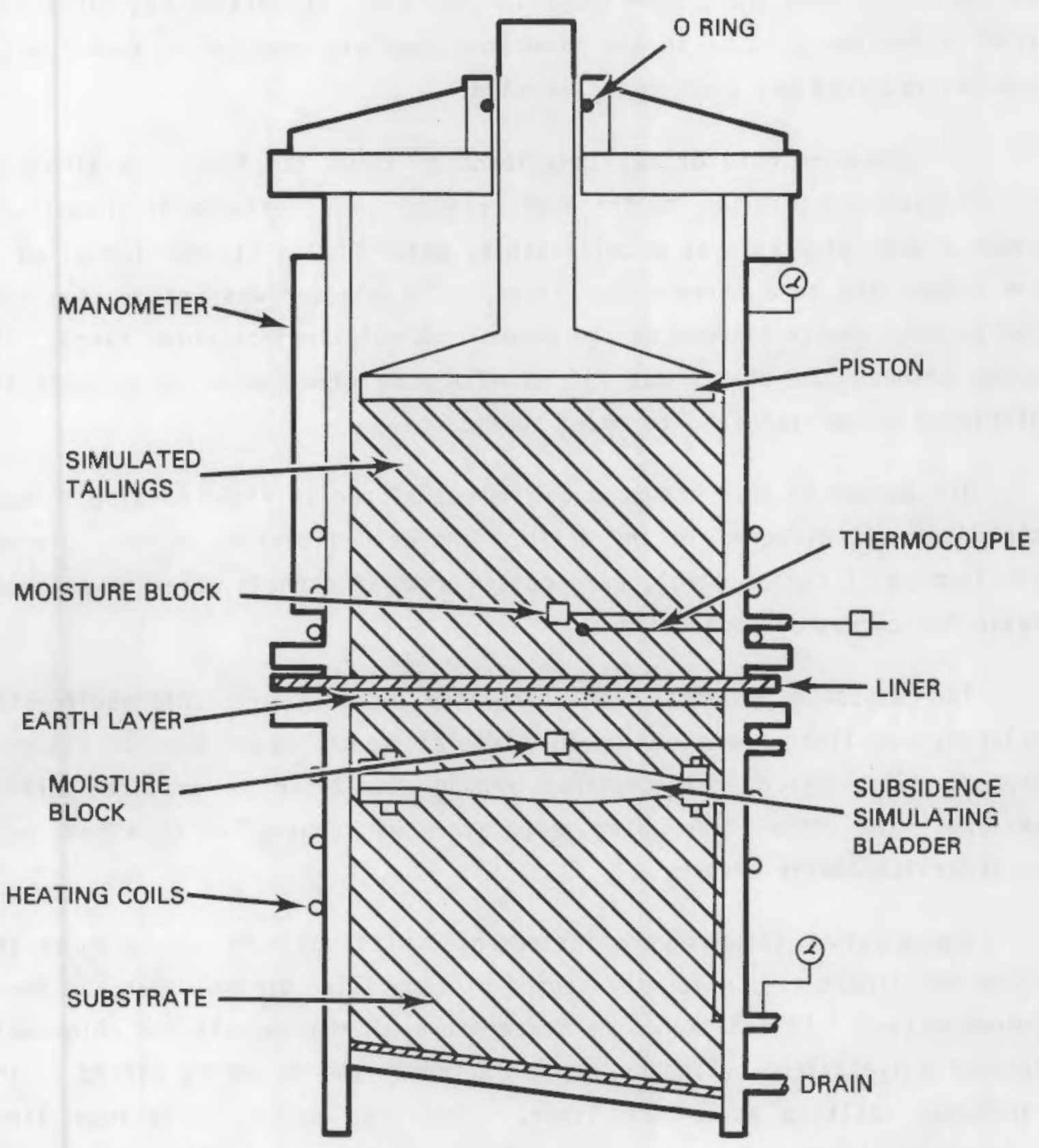

FIGURE 8. Construction Details of Liner Exposure Columns 
Impoundment characteristics that had to be simulated in the exposure columns included forces exerted by the tailings above the liner; the pressure head of tailings leachate in the impoundment, if any; and subsidence of the supporting substrate. The weight of the tailings above the liner was duplicated by applying a representative force on the simulated tailings in the column with the piston shown in Figure 8. The piston was fabricated from perforated plating so the simulated leachate could flow freely while the desired load was applied to the sand.

The pressure head of tailings leachate above the liner was simulated by applying gas pressure to the test cylinder. The effects of ground subsidence were simulated by a collapsible, water-filled bladder installed in the supporting sand beneath the liner. The bladder was constructed from two hypalon sheets clamped at the edges with polyvinyl-chloride rings. The space between the sheets was filled with sufficient water to produce the displaced volume required to model subsidence.

The bottom of each exposure column was sloped so fluid passing through the liner was directed to the drain. The welded bottoms of the columns, constructed of carbon steel, were coated with an asphalt layer or an epoxy resin for corrosion protection.

The exposure columns containing the asphalt and chlorosulfonated polyethylene liners were heated by circulating hot water through external copper coils, which were centered around the liner to provide uniform heating. The circulating water temperature was controlled by a heat pump or immersion heater system.

The expostire columns were instrumented with moisture blocks above and below the liners to monitor the degree of saturation of the upper and lower column halves. In addition, the temperature of the asphalt and chlorosulfonated polyethylene cylinders was monitored by thermocouples placed in the simulated tailings above the liner. This was necessary because liner temperature is one of the most important variables controlling the oxidation rate and these data were required to predict liner aging. 
SCREENING TEST RESULTS

The liners and supporting soil were sandwiched in the exposure columns between simulated tailings and a sand layer. The tailings above the liner in each of the columns were simulated with Wedron washed silica sand, Size No. 70, which was selected because it resembled the particle size distribution of samples removed from the New Rifle, Colorado, tailings pile (see Figure 2, Hartley et al. 1980) and it would not affect the chemical reactions between the leachate and the liner. The same sand was used beneath the liners to minimize absorption of leachate that passed through them.

Just below the asphalt and chlorosulfonated polyethylene liners, a $0.5-\mathrm{cm}$ layer of soil was installed to subject the bottom surface of the liner to realistic conditions. Also, in every case the centerlines of the subsidence bladder and moisture blocks were placed $9.5 \mathrm{~cm}$ and $3.2 \mathrm{~cm}$ below the liners, respectively. In each of the cylinders, the sand beneath the liner was compacted with a vibrating rod.

The permeability values, $k$, calculated for the testing cycles are presented in Figures 9 and 10. These values were calculated using the Darcy equation for saturated flow:

$$
k=\frac{\Delta V L}{A P t}
$$

$$
\text { where } \begin{aligned}
k & =\text { permeability, } \mathrm{cm} / \mathrm{s} \\
V & =\text { change in fluid volume, } \mathrm{cm}^{3} \\
L & =\text { liner thickness, } \mathrm{cm} \\
A & =\text { surface area of liner, } \mathrm{cm}^{2} \\
P & =\text { pressure difference across the liner, } \mathrm{cm} \mathrm{H}_{2} \mathrm{O} \\
\mathrm{t} & =\text { permeability cycle duration, } \mathrm{s} .
\end{aligned}
$$

Oepending on the permeability of the liner, $\Delta V$ was measured by the volume of fluid collected below the liner during a permeability measurement cycle or by the loss in volume above the liner as measured by the manometer. Pertinent data for each permeability test $(\Delta V, P, t$, etc.) are listed in Appendix A. 


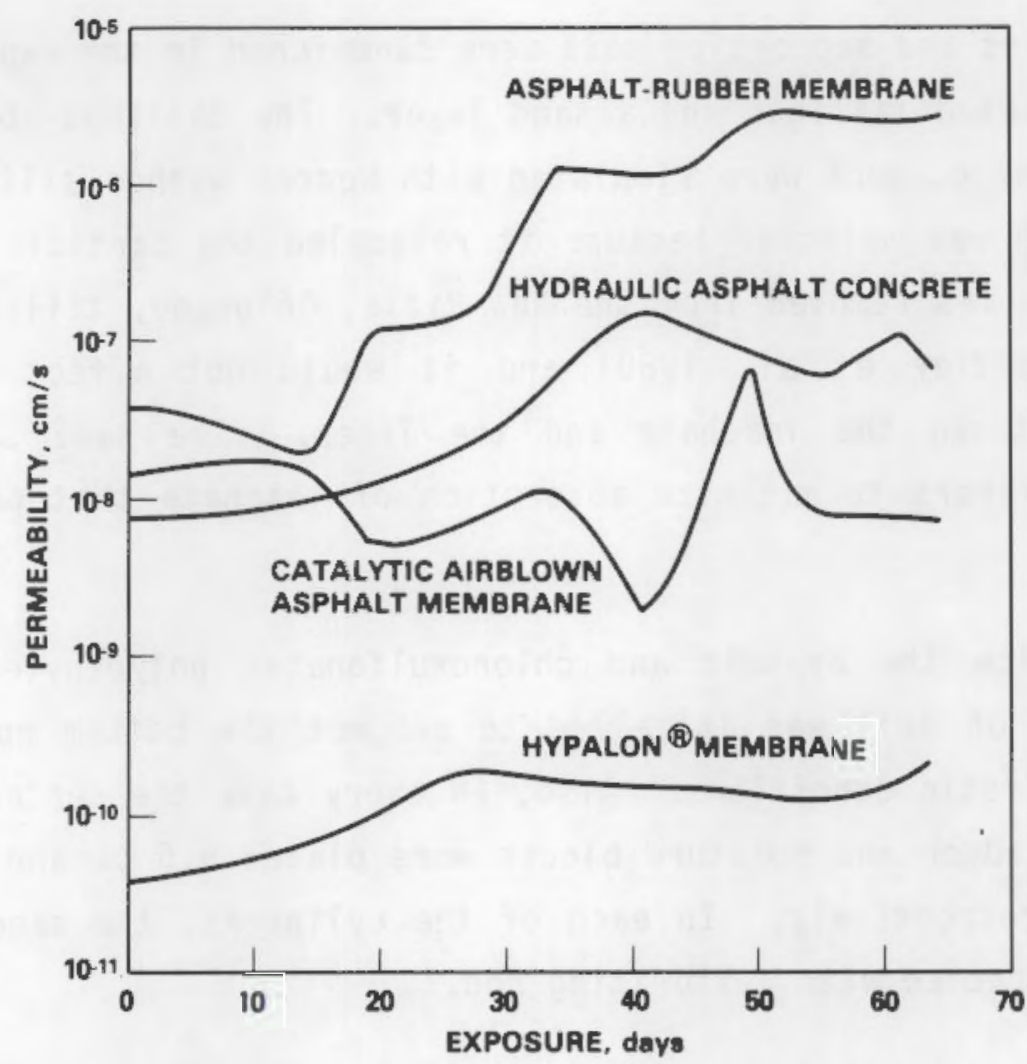

FIGURE 9. Permeability Performance of Asphalt and Hypalon Liners During Laboratory Testing Period

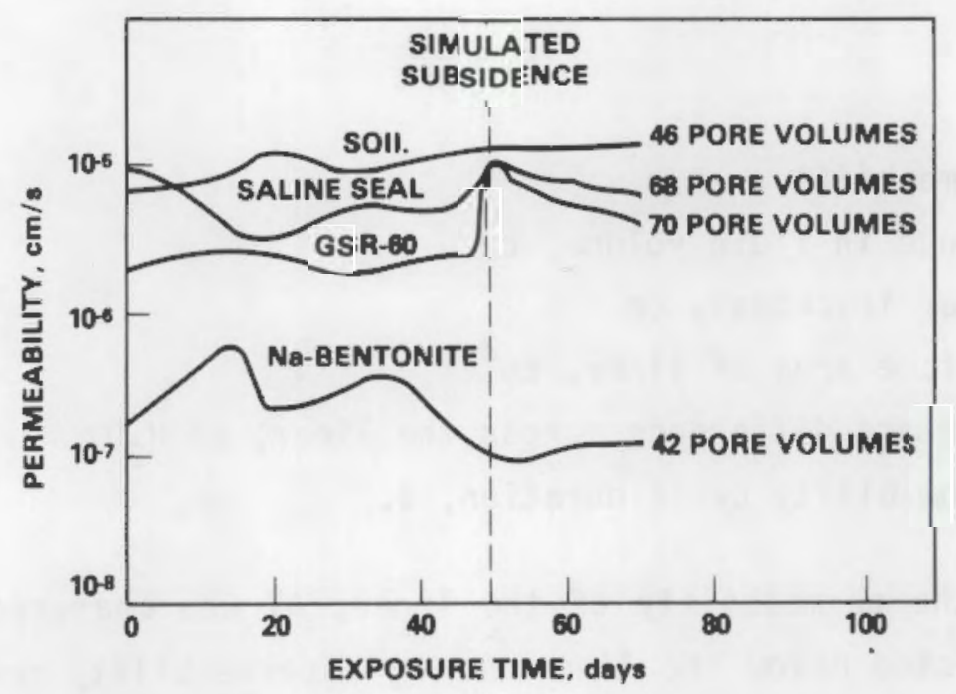

FIGURE 10. Permeability Performance of Clay-Amended and Natural Soil During Laboratory Testing Period 
The change of permeability with exposure time is a good indication of the relative stability of the liners; however, when evaluating the effectiveness of liner materials of different thicknesses, it is not sufficient to compare permeability alone. In the field, a liner such as a soil amended with bentonite 10 to $20 \mathrm{~cm}$ thick need not be as impermeable as a thin membrane to be an effective leachate barrier. Therefore, the liners were also compared by averaging the permeabilities from the final 30 days and dividing the liner field thickness. The $\mathrm{K} / \mathrm{L}$ factors, called the permeability effectiveness factors, for all liners are listed in Table 10.

TABLE 10. Anticipated Field Liner Permeabilities

\begin{tabular}{|c|c|c|c|}
\hline Liner & $\mathrm{K}, \mathrm{cm} / \mathrm{s}$ & L, $\mathrm{cm}$ & Factor, $K / L, S^{-1}$ \\
\hline Asphalt Concrete & $7\left(10^{-8}\right)$ & 10 & $7\left(10^{-9}\right)$ \\
\hline Hypaton & $2\left(10^{-10}\right)$ & 0.12 & $2\left(10^{-9}\right)$ \\
\hline $\begin{array}{l}\text { Asphalt Rubber } \\
\text { Membrane }\end{array}$ & $4\left(10^{-6}\right)$ & 0.8 & $5\left(10^{-6}\right)$ \\
\hline $\begin{array}{l}\text { Catalytic Airblown } \\
\text { Membrane }\end{array}$ & $7\left(10^{-9}\right)$ & 0.9 & $8\left(10^{-9}\right)$ \\
\hline Sodium Bentonite & $1\left(10^{-7}\right)$ & 10 & $1\left(10^{-8}\right)$ \\
\hline Saline Seal 100 & $8\left(10^{-6}\right)$ & 10 & $8\left(10^{-7}\right)$ \\
\hline GSR-60 & $6\left(10^{-6}\right)$ & 10 & $6\left(10^{-7}\right)$ \\
\hline Soil (as a liner) & $1\left(10^{-5}\right)$ & 10 & $1\left(10^{-6}\right)$ \\
\hline
\end{tabular}

Asphalt and Synthetic Liners

With the exception of the CAA membrane, the permeability of each of the asphalt and synthetic liners increased during the laboratory testing period. A discussion of the performance of each of these liners follows; and the pressure, $\mathrm{pH}$, and temperature of the exposure columns during the testing period are plotted in Figures 11,12 , and 13 , respectively. 

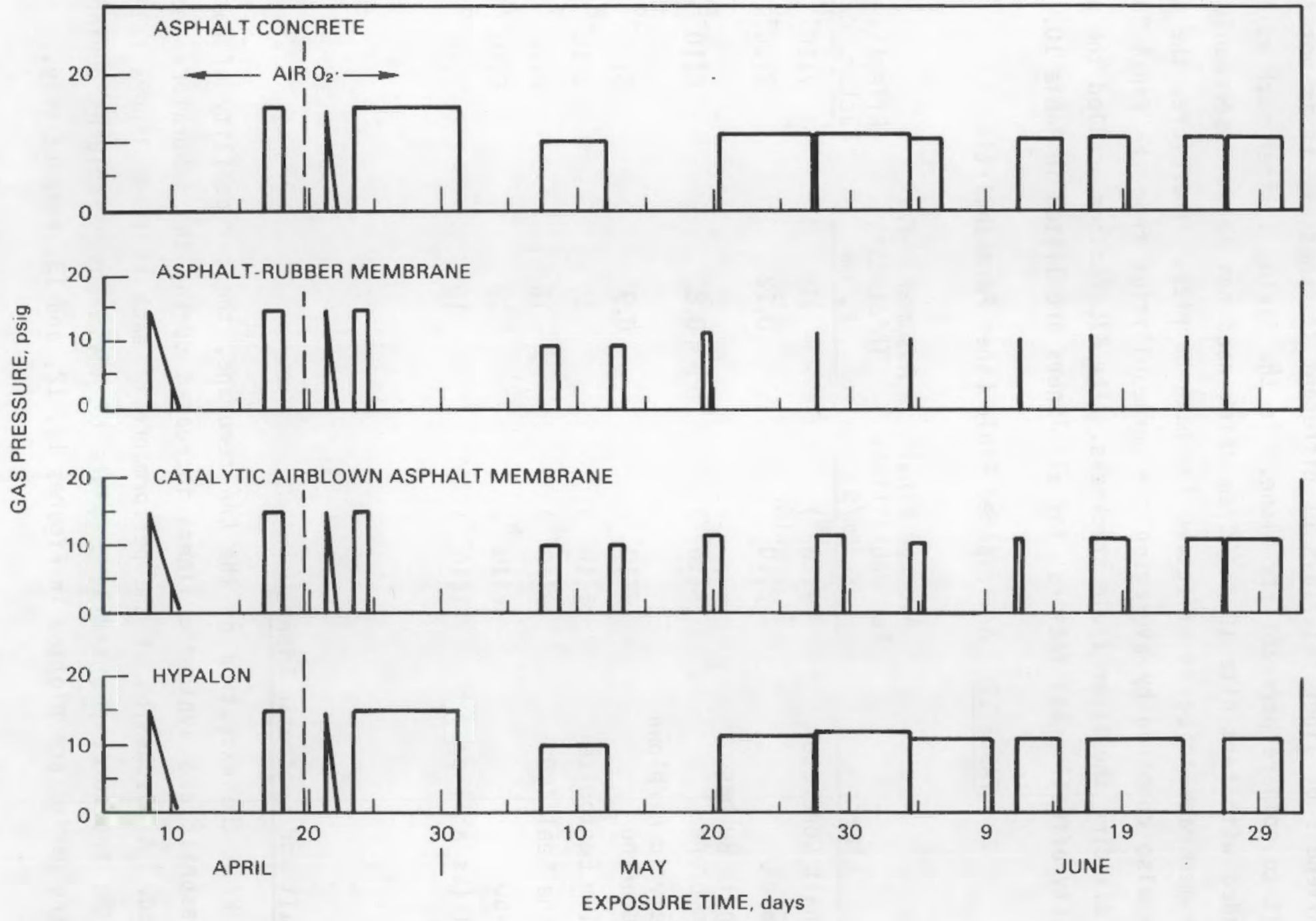

FIGURE 11. Oxygen Pressure to Which Asphalt and Synthetic Liners Were Exposed During Testing 

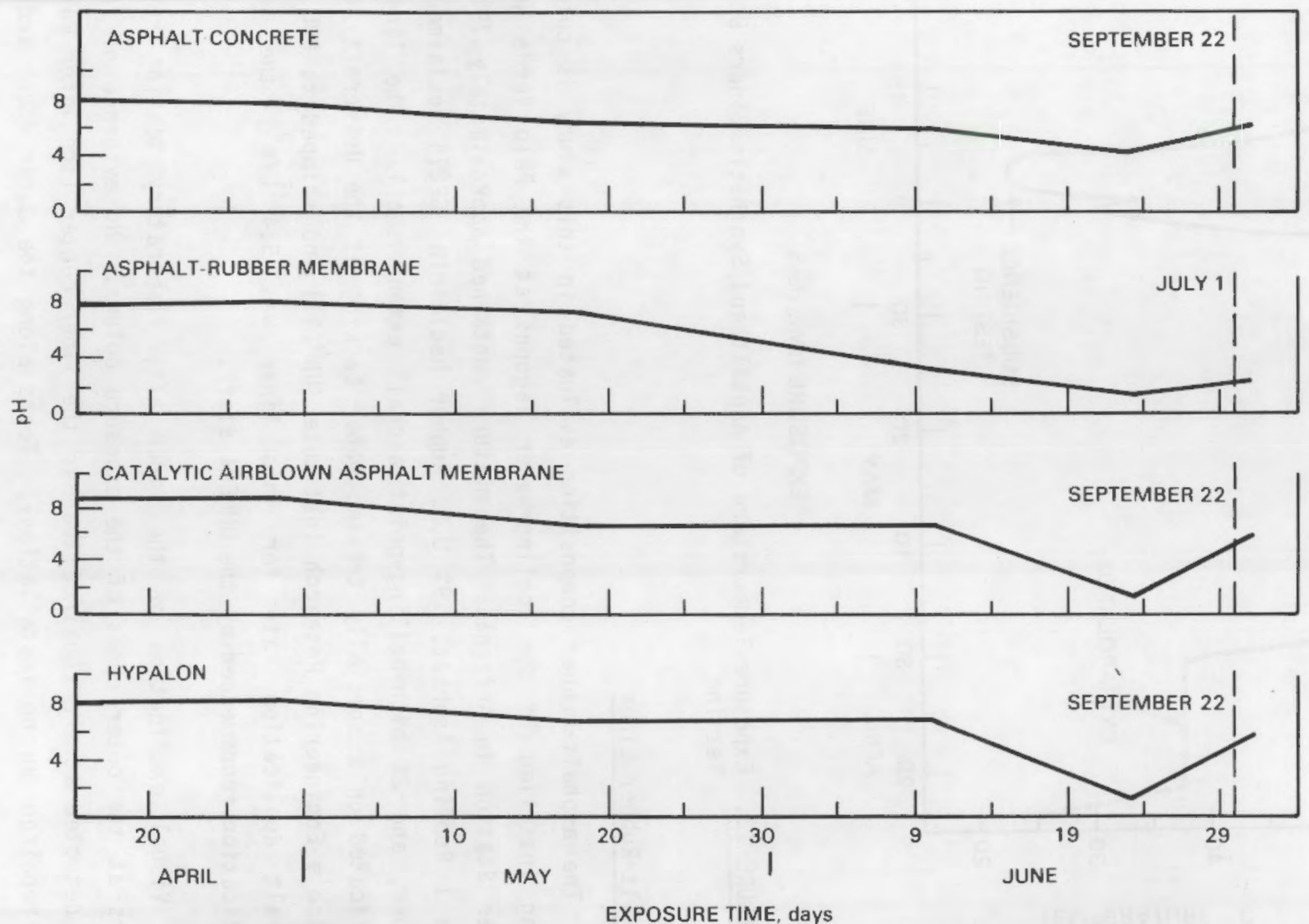

FIGURE 12. Acidity Concentration During Testing of Asphalt and Synthetic Liners (includes pH values measured after test completion, June 30 ) 


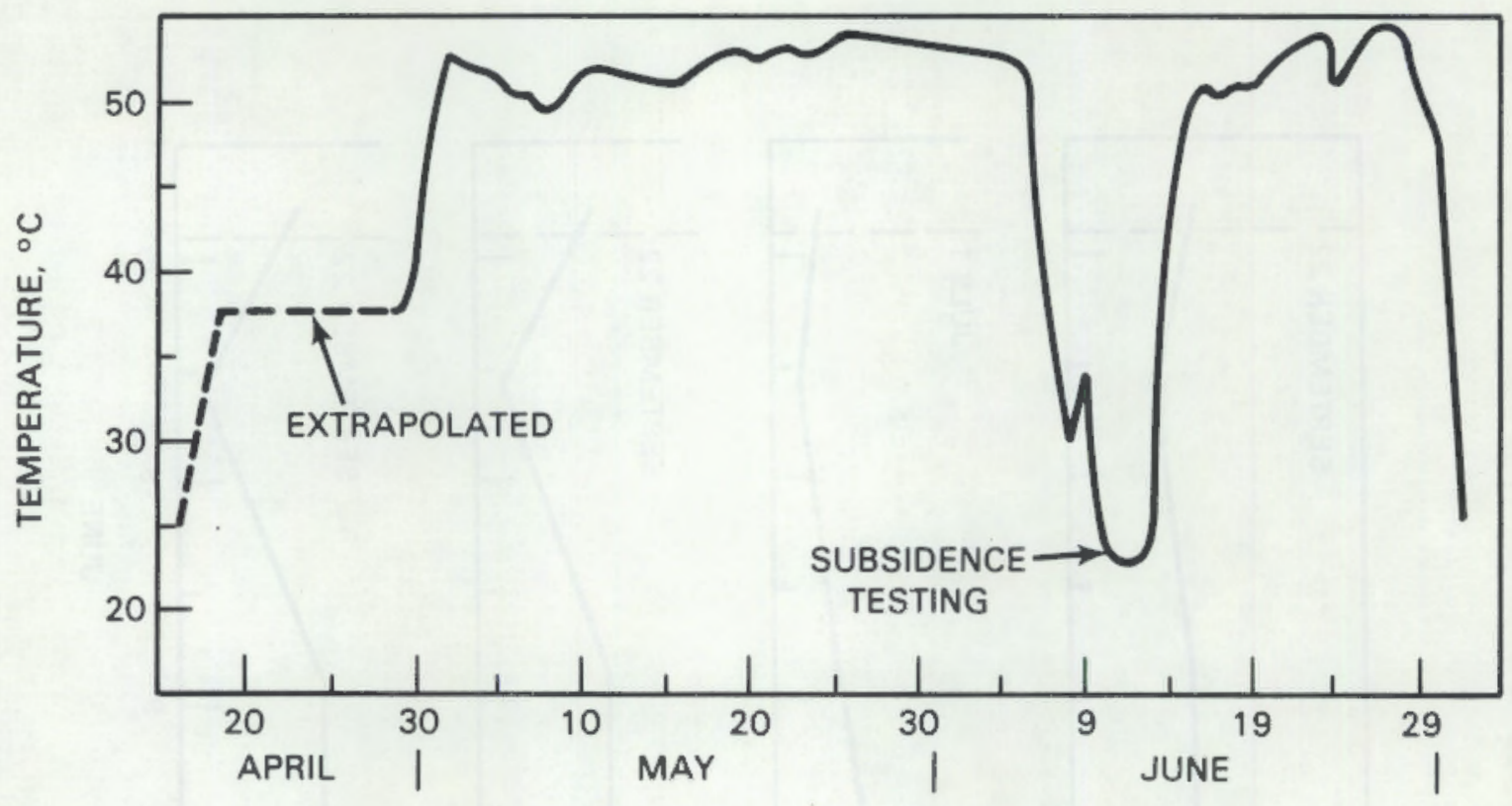

EXPOSURE TIME, days

FIGURE 13. Exposure Temperature of Asphalt and Synthetic Liners During Testing

Asphalt-Rubber Liner

The asphalt-rubber composition evaluated in this study is currently being installed in the cooling-water lagoons at the Palo Verde Nuclear Power Station in Arizona. The mixture contained approximately 79\% ASTM Type 1 Roofing Asphalt, 19\% U.S. Rubber Reclaiming GP274 reclaimed tire rubber, and 2\% high-boiling-point asphalt extender oil. The liner was fabricated on a specially treated paper backing at the University of New Mexico's Engineering Research Institute (UNMERI) and shipped to PNL. The asphalt application rate for this liner was $5.4 \mathrm{~L} / \mathrm{m}^{2}$, the minimum application recommended by the UMMERI staff.

Visual examination of the liner after laboratory testing revealed tears at the outer edges of the exposure column. No evidence of failure was detected at the repaired tear in the liner center (the liner was torn and repaired during installation). Tears along the cuter edges indicated 
insufficient elasticity and strength when the subsidence bladder was collapsed near the end of the test. This event also produced the permeability increases shown in Figure 9. Another permeability increase was observed subsequent to acid addition to the tailings leachate and may indicate that the elastomer used in this liner is not compatible with highly acidic environments.

\section{Hydraulic Asphalt Concrete}

The asphalt concrete liner was produced by installing two $4.5-\mathrm{cm}$ thick lifts of admixed asphalt above a cationic asphalt emulsion tack coat. Asphalt emulsion was also applied to the cylinder walls to bond the liner to the exposure cylinder. An AR4000 asphalt cement, containing $10 \%$ asphalt and $90 \%$ aggregate, was used to fabricate this liner. Specifications are provided in Appendix B.

The asphalt and aggregate were heated to $200^{\circ} \mathrm{C}$ and mixed in two $12.5-$ $\mathrm{kg}$ batches. After mixing, the admixture was reheated to $200^{\circ} \mathrm{C}$ and installed in the exposure column. Individual lifts were poured into the cylinder, leveled, and partially compacted by hand; then they were compressed to 78 psig in a hydraulic press. This pressure was used because it represents the compactive force developed in a 90-blow Marshall compaction procedure and because it represents the compressive action of a 5-ton vibratory roller. The lower asphalt concrete lift was allowed to cool for 30 minutes before the upper lift was installed.

The permeability of this liner increased early in the laboratory tests but stabilized at $10^{-7} \mathrm{~cm} / \mathrm{s}$. This result suggests that one (or more) of the liner components--either the asphalt cement, the asphalt emulsion used to seal the edges, or the aggregate--was degraded by the accelerated aging testing; however, comparison of the effectiveness of the liner permeability with that of other liners shows this material to be acceptable.

As expected, this asphalt concrete liner did not demonstrate a permeability increase in the subsidence test. No significant effect was expected because of the inherent strength of the $7.6-\mathrm{cm}$ thick liner. 
Catalytic Airblown Asphalt Membrane

The catalytic airblown asphalt (CAA) membrane, a hot-sprayed liner, was also applied in two lifts. Asphalt was placed in a heated reservoir and pressurized with nitrogen, which forced the CAA through a manually operated spray gun. The first lift was sprayed directly on the soil layer; the second lift was sprayed from an opposing direction to assure complete coverage. The application rate for the completed liner was $6.8 \mathrm{~L} / \mathrm{m}^{2}$, and the CAA was sprayed at a temperature of $205^{\circ} \mathrm{C}$. The specifications of this liner are listed in Appendix B.

Permeability measurements generated during tests of this liner displayed the greatest amount of data scatter. This variability is attributed to two factors. First, excess tailings leachate that collected in the bottom of the cylinders between permeability measurement cycles was included in the total cylinder volume change. Second, the liner was used as a gasket to seal the flanged connection at the center of the exposure column. Occasionally, leaks developed at this connection as the asphalt flowed to relieve stress applied by the bolts holding the flanges together. Gas pressure in the column pushed the simulated tailings leachate above the liner through these leaks. The low and high permeabilities measured before and after the subsidence test were caused by these mechanisms and are of questionable value.

Despite the erroneous readings on a few data points, enough permeabi1$i$ ty data were collected to demonstrate the stability of the CAA membrane when exposed to accelerated conditions. This was the only material in the asphalt and synthetic liner category that exhibited a permeability at the end of the exposure period that was not greater than the initial measurements. In addition, the permeability effectiveness factor $\left(K / L=8 \times 10^{-9}\right)$ was comparable to the best of the liner materials tested. No visible degradation was observed. 


\section{Chlorosulfonated Polyethylene}

To represent geomembrane liners, a sheet of $1.5-\mathrm{mm}$ chlorosulfonated polyethylene (hypalon) was placed above the $0.5-\mathrm{cm}$ soil layer and sealed to the cylinder with silicone rubber cement. This liner consistently produced the lowest permeability measured; however, because of $i$ ts thickness, the permeability effectiveness factor, $K / L$, of this membrane was within the same order of magnitude as the CAA membrane and the hydraulic asphait concrete liner.

The subsidence test produced no permeability change and no visible degradation was observed, but salt deposits were noted at the outside edge of the liner. (This liner was also used as the center gasket; therefore, the outer edge was outside of the exposure column.) The salt deposits were attributed to leachate wicking along the fiber reinforcement. A siight increase in permeability with time was noted, indicating some decrease in stability.

\section{Clay Liners}

The four clay liners did not achieve the same initial permeabilities al though each was prepared with the same 1:10 weight ratio of clay to soil. The differences in permeabilities were caused by several factors. A procedural error occurred during installation when the Saline Seal 100 and the GSR 60 liners were not moistened before compaction. In addition, the desired initial compaction could not be achieved because of fluid motion in the subsidence bladder. Finally, there were differences in particle size between the natural sodium bentonite and the polymer-treated bentonites. The latter were more granular and inhibited the passage of the polymertreated bentonites into smaller pores of the soil. A thicker liner installed under field conditions should eliminate these problems. The pressure and $\mathrm{pH}$ histories of these liners are plotted in Figures 14 and 15. 

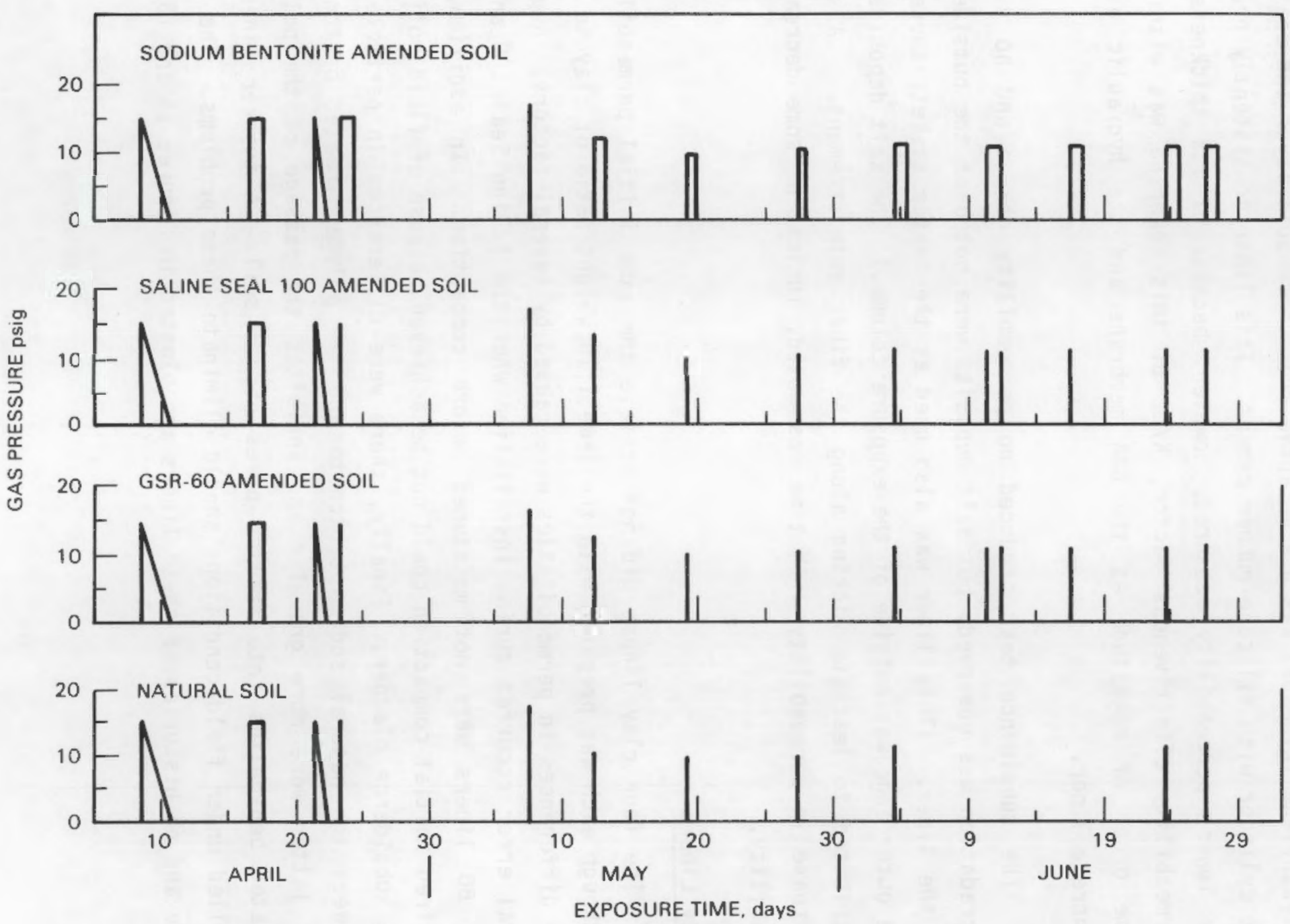

FIGURE 14. Pressure History During Testing of Clay Liners 

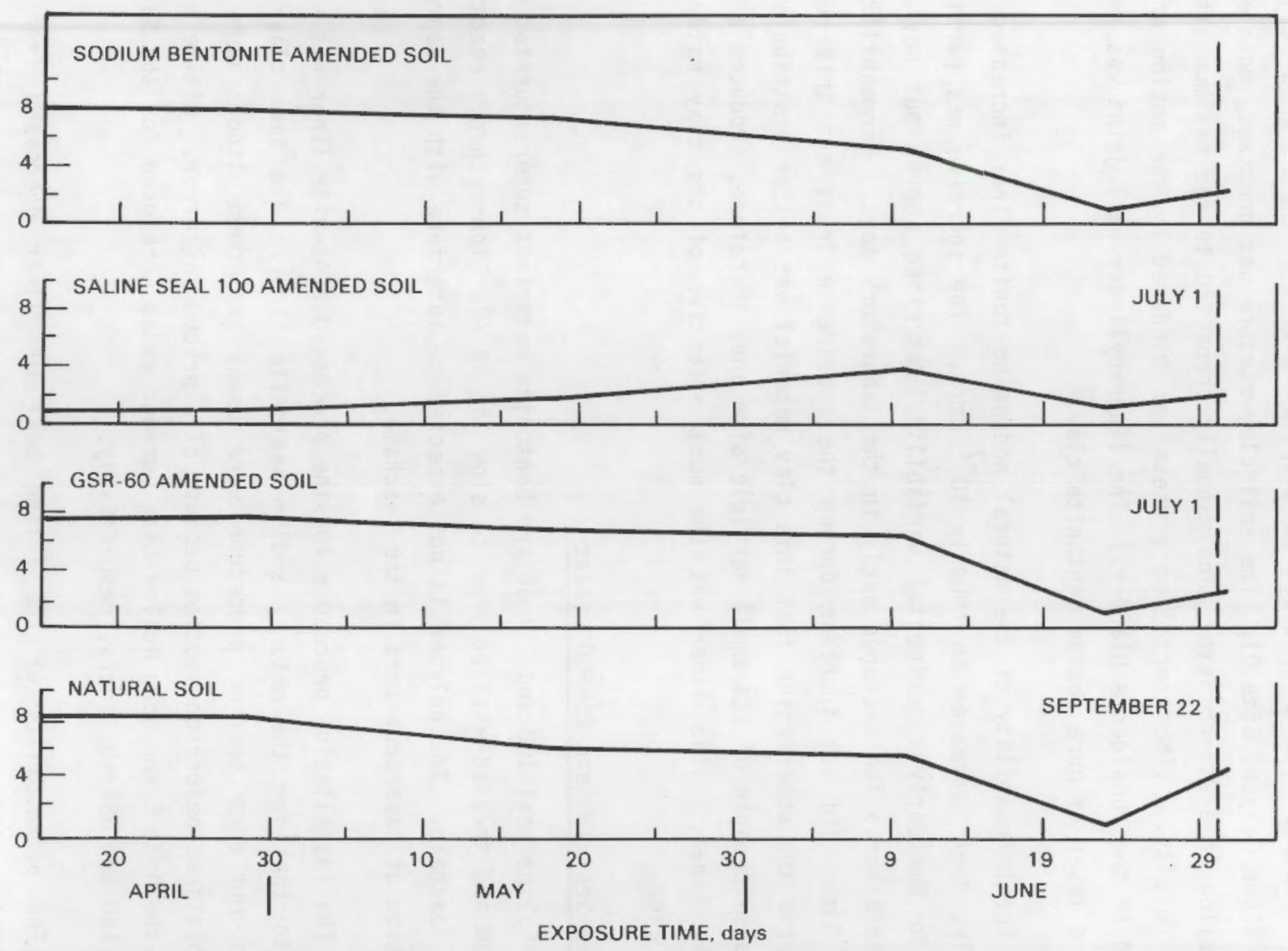

FIGURE 15. Acidity Concentration Ouring Testing of Clay Liners (includes pH values measured after test completion, June 30) 
Sodium Bentonite Liner

Natural sodium bentonite clay was mixed at a weight ratio of $1: 10$ with a soil sample from a prospective disposal site for the Durango Tailings Pile (Bodo Canyon, Site D). The soil-clay mixture was premixed, moistened, installed in a $2.5-\mathrm{cm}$ layer, and manually compacted to the maximum attainable density. (The compaction process was hindered by the motion of the fluid in the subsidence bladder.) The liner-cylinder wall joint was sealed with a layer of pure sodium bentonite clay.

The permeability of the natural sodium bentonite liner increased initially, then decreased to roughly $10^{-7} \mathrm{~cm} / \mathrm{s}$. The increase was partially due to the early experimental variability described above, but may also indicate $\mathrm{Na}-\mathrm{Ca}$ ion exchange early in the laboratory test. Permeability of this liner did not increase during the subsidence test; and this result supports the observation that this clay material was better distributed in the soil because of its small particle size and, therefore, produced a more stable liner. This liner was the most effective of the clay materials evalua ted.

Saline Seal 100 and GSR-60 Liners

Saline Seal 100 and GSR-60 are bentonite materials supersaturated with sodium and treated with polymer to slow the $\mathrm{Na}^{+} / \mathrm{Ca}^{+2}$ ion-exchange reactions with leachate. The polymer is not expected to interfere with the coprecipitation of hazardous ions in the leachate.

The installation procedure for the processed bentonite liner was similar to that for the natural sodium bentonite liner. The same clay/soil ratio and edge sealing procedure were used, but these liners were not premoistened before compaction because of a procedural error. Native soils from the Bodo $E$ and Long Hollow East Durango sites were used for the Saline Seal 100 and GSR-60 liners, respectively.

The permeability of the Saline Seal 100 liner decreased from $i$ ts original, relatively high value and reached a plateau of $6 \times 10^{-6} \mathrm{~cm} / \mathrm{s}$. 
It remained at this level until the subsidence test, when the permeability increased as the liner was disrupted. A slow decrease in permeability after subsidence indicated that this liner exhibits some self-healing characteristics.

The permeability of the GSR-60 liner was nearly constant $3 \times 10^{-6} \mathrm{~cm} / \mathrm{s}$ from the beginning of the laboratory tests until the simulated subsidence. A large permeability increase was observed during the subsidence test because this membrane was also disrupted; and again, the clay demonstrated both a tendency to seal the liner following a disruptive incident and to reduce permeability following the subsidence test.

\section{Natural Soil Liner}

A control liner of soil from the Bodo $E$ Durango disposal site was installed in one of the exposure columns. This liner was dry-compacted to a $2.5-\mathrm{cm}$ thickness and sealed to the column with the sodium bentonite liner installation technique. The earth was passed through a $0.6-\mathrm{cm}$ sieve to remove large rocks, which could have damaged a thin liner. No subsidence bladder was installed in this cylinder.

The natural soil liner was installed as a control to monitor any permeability change resulting from degradation. This liner displayed a generally increasing permeability trend throughout the tests.

\section{Liner Costs}

Installation costs were also compared. Charges were estimated by contacting vendors and installation contractors, who were asked to provide pricing information based on a $210,000-\mathrm{m}^{2}$ impoundment at Clive, Utah. Estimates included material, transportation, and installation costs for each liner; however, variations in subsurface preparation requirements for these liners were not included. Cost estimates are presented in Table 11. 
TABLE 11. Costs of the Candidate Liner Materials

Liner

Asphalt concrete

Chlorosulfonated polyethylene

Asphalt-rubber

Catalytic airblown asphalt

Saline Seal 100

GSR-60

Sodium bentonite
Installation Rate

Two 5-cm lifts with cationic asphalt $t_{2}$ emulsion tack coat $\left(3 \mathrm{~L} / \mathrm{m}^{2}\right)$
Installed Costs

$\$ 9.20 / \mathrm{m}^{2}$

$\$ 5.90 / \mathrm{m}^{2}$

$5.4 \mathrm{~L} / \mathrm{m}^{2}$

$\$ 2.40 / \mathrm{m}^{2}$

$9 \mathrm{~L} / \mathrm{m}^{2}$

$\$ 2.60 / \mathrm{m}^{2}$

$20 \mathrm{~kg} / \mathrm{m}^{2}$

$\$ 4.70 \mathrm{~m}^{2(\mathrm{a})}$

$20 \mathrm{~kg} / \mathrm{m}^{2}$

$\$ 2.70 / \mathrm{m}^{2(a)}$

$20 \mathrm{~kg} / \mathrm{m}^{2}$

$\$ 2.30 / \mathrm{m}^{2(\mathrm{a})}$

(a) Assumes that $10,2-\mathrm{cm}$ (4-in.) layer of clay and soil is installed with soil at $\$ 5.20 / \mathrm{m}^{3}$.

Conclusions

The screening tests produced the following findings:

- Expected gamma irradiation in the field will not impair liner performance over the 1000-year period.

- Simulated subsidence indicated that asphalt concrete, chlorosulfonated polyethylene, sodium bentonite-amended soil, and probably the catalytic airblown membrane will yield the best performance.

- Hydraulic asphalt concrete, catalytic airblown asphalt, chlorosulfonated polyethylene, and sodium bentonite-amended soil are the liners with the best potential for field performance (as determined by permeability effectiveness factors, $K / L$ ).

- Catalytic airblown asphalt and soil amended with natural sodium bentonite are the most stable of the liners tested (as determined by the change of permeability with time). 
- Initial permeability increases measured on the soil and natural sodium bentonite liners may indicate that rapid $\mathrm{Na}-\mathrm{Ca}$ ion exchange is occurring. Since the overall performance was not affected, however, it may be assumed that the resulting shrinkage occurs vertically. This was confirmed by determining the extent of the ion-exchange reaction in subsequent studies.

- Asphalt-rubber and catalytic airblown asphalt are the least expensive of the asphalt and synthetic materials to install.

- Natural sodium bentonite and GSR-60 are the least expensive of the clay materials to install.

Based on the laboratory test results, expected field effectiveness and cost analysis, catalytic airblown asphalt and sodium bentonite-amended soil were selected for the fiscal year 1981 field studies and subsequent laboratory studies.

\section{CONTROLLED AGING TESTS OF THE ASPHALT MEMBRANE}

With the selection of the catalytic asphalt membrane as the most prospective liner in its group, further studies were required to quantify $i$ ts expected performance lifetime. It was necessary to determine the penetration of reaction into the liner as well as the activation energy and dependency of $\mathrm{pH}$ and oxygen concentration on reaction rate; therefore, a series of exposure conditions were imposed.

\section{Exposure Conditions}

To select three values for each of the three accelerated aging parameters, a $3 \times 3 \times 3$ testing matrix was used, resulting in 27 liner exposure tests. Since only eight exposure columns were available, however, a statistical method was used to select only key parameter combinations in the $3 \times 3 \times 3$ testing matrix. This method, known as the fractional factorial matrix, requires measurements at 11 points rather than 27 (see Figure 16). With this statistical method, the high and low values of each 

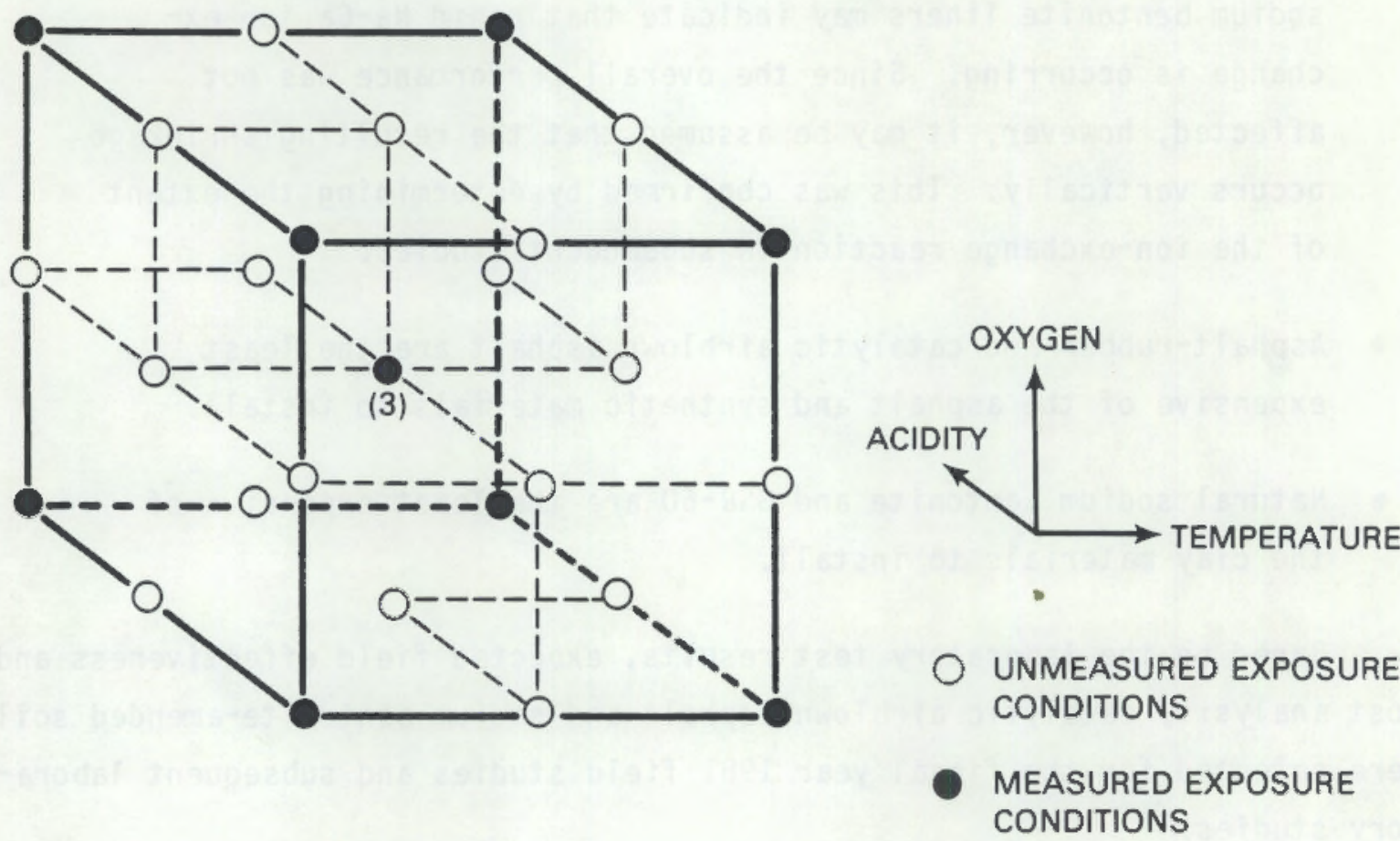

FIGURE 16. Fractional Factorial Matrix

parameter are selected for testing; and the liner is subjected to three exposure tests at the intermediate values of all three parameters to provide a statistical variation of the data. With eight exposure columns available, the 11 measurements could be made in two series of tests, leaving spares to rerun any cases that might be necessary.

The glycol cooling/heating coils on the exterior of the exposure columns were used to control temperature for the various points on the fractional factorial matrix. Intermediate and high temperatures (35 and $50^{\circ} \mathrm{C}$ ) were maintained by electric heaters immersed in glycol that was continually recirculated through the heat transfer coils of the respective columns. The intermediate- and high-temperature cases were selected on the basis of anticipated reaction rate constants and the desire to avoid chemical reactions in the asphalt above $50^{\circ} \mathrm{C}$ that might not occur normally. The $20^{\circ} \mathrm{C}$ ambient temperature, which was maintained in the column by the heat pump, represented the equivalent ground temperature that might be expected below a uranium mill tailings pile. 
The range of oxygen partial pressures for accelerated aging was based on ambient conditions, the maximum pressure rating of the exposure column, and an arithmetic average between the two. Therefore, $0.21,1$, and $1.7 \mathrm{~atm}$ were selected as the exposed oxygen partial pressures with a total pressure of $1.7 \mathrm{~atm}$ in all cases. The prescribed partial pressure of oxygen was maintained in the exposure column by two flow control valves from oxygen and nitrogen supply tanks. The intermediate and ambient oxygen partial pressures were more difficult to maintain because the flow of oxygen and nitrogen had to be balanced to match the leak rate of the exposure columns.

The asphalt liner was continually exposed to acidic leachate of the composition listed in Table 12. Acidity levels were maintained by periodic leachate sampling directly above the liner. As the $\mathrm{pH}$ slowly increased throughout the exposure period, additional sulfuric acid was introduced into the exposure column. The $\mathrm{pH}$ tended to increase because the leachate interacted with the exposure column, the simulated tailings, and possibly the asphalt liner itself.

\section{TABLE 12. Simulated Tailings Leachate Composition}

\begin{tabular}{lc} 
Chemical & Concentration, $\mathrm{g} / \mathrm{L}$ \\
\cline { 2 - 2 } $\mathrm{CaSO}_{4}$ & 500.0 \\
$\mathrm{CaCO}_{3}$ & 18.1 \\
$\mathrm{MgSO}_{4}$ & 8.6 \\
$\mathrm{Na}_{2} \mathrm{SO}_{4}$ & 7.4 \\
$\mathrm{NaCl}$ & 7.4 \\
$\mathrm{Fe}_{2}{ }_{3}$ & 2.4 \\
$\mathrm{NaCO}_{3}$ & 2.3 \\
$\mathrm{Al}_{2} \mathrm{O}_{3}$ & 1.2
\end{tabular}


This situation posed a difficult control problem and required that various conditions be rerun in the second series of tests. The base case of $\mathrm{pH}=2.5$ was selected as the lowest measured $\mathrm{pH}$ from a variety of batch leaching samples of uranium mill tailings from Shiprock, Durango, and Salt Lake City. The pH values selected for the accelerated conditions were 2.0 and 1.5 .

The column leaching studies indicated that the initial pore volume of leachate passing through Shiprock tailings had a pH of 1.8 and increased to higher $\mathrm{pH}$ values with subsequent pore volumes of leachate (Relyea and Martin 1982). The concern over maintaining accurate $\mathrm{pH}$ levels proved to be inconsequential, however, since it was discovered during asphalt liner analysis that acidity levels have an unmeasurable effect on the extent of aging.

Appendix $C$ contains the ph curves measured for all asphalt samples during both series of tests. Temperature and oxygen exposure conditions are also given.

Permeability

Permeability was used to measure the immediate effectiveness of the asphalt liner. Because of the low permeability of the asphalt liner $\left(10^{-9}\right.$ $\left.10^{-10} \mathrm{~cm} / \mathrm{s}\right)$, measurable volumes of leachate were collected infrequently; therefore, permeability was determined by changes in manometer levels during the exposure period. As indicated by the manometer, the time required for a permeability measurement was approximately 2 weeks.

Due to the chemical durability of the asphalt, accelerated aging conditions produced no measurable effect on permeability with time, even after the subsidence bladder was collapsed. Figure 17 represents the permeability measurements for three liners at normal, intermediate, and highly accelerated conditions. The flatness of the curves indicates that permeability cannot be used to assess the extent of aging in the asphalt liner and that additional analytical techniques are necessary to make such a determination. The permeability curves do, however, indicate that the 


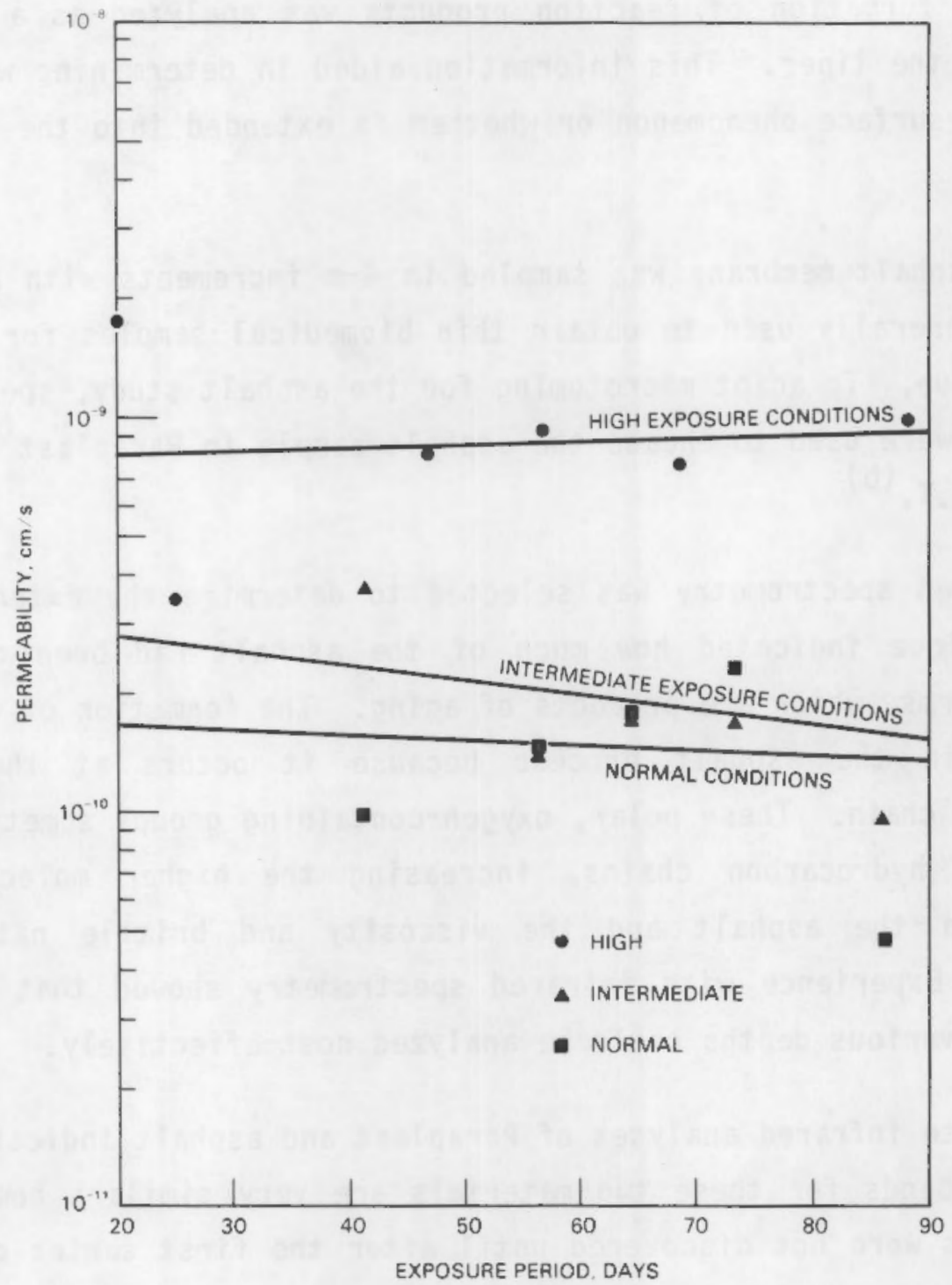

FIGURE 17. Permeability of Asphalt Liner at Norma 1, Intermediate, and Highly Accelerated Conditions

performance of the asphalt liner was not affected even for under maximum accelerated aging conditions. Permeability data for each run are given in Appendix $C$. 
Analytical Results

Since premeability was unsatisfactory as a measure of the extent of aging, the formation of reaction products was analyzed as a function of depth into the liner. This information aided in determining whether aging was a near-surface phenomenon or whether it extended into the depth of the asphalt.

The asphalt membrane was sampled in 4-m increments with a microtome, which is generally used to obtain thin biomedical samples for analysis of animal tissue. To adapt microtoming for the asphalt study, special casting techniques were used to encase the asphalt sample in Paraplast ${ }^{(a)}$ (paraffin wax) or O.C.T. (b)

Infrared spectrometry was selected to determine the extent of aging. This technique indicated how much of the asphait had been converted to carbonyl forms, which are products of aging. The formation of carbonyls is important in the asphalt process because it occurs at the end of a hydrocarbon chain. These polar, oxygen-containing groups sometimes combine with other hydrocarbon chains, increasing the higher molecular weight fraction in the asphalt and the viscosity and brittle nature of the membrane. Experience with infrared spectrometry showed that all asphalt samples at various depths could be analyzed cost-effectively.

Separate infrared analyses of Paraplast and asphalt indicated that the absorption bands for these two materials are very similar; however, their similarities were not discovered until after the first series of tests had been cast and microtomed for analysis. For this reason, the second series of asphalt samples was cast in 0.C.T., a material with absorption bands quite different from asphalt. Figure 18 shows the absorption bands and

(a) Paraplast is a registered trademark of the Lancer Division, Snerwood Medica 1, St. Louis, Missouri.

(b) 0.C.T. (Optimal Cutting Temperature) is a registered trademark of the Labtek Division, Myles Laboratory, Naperville, Illinois. 

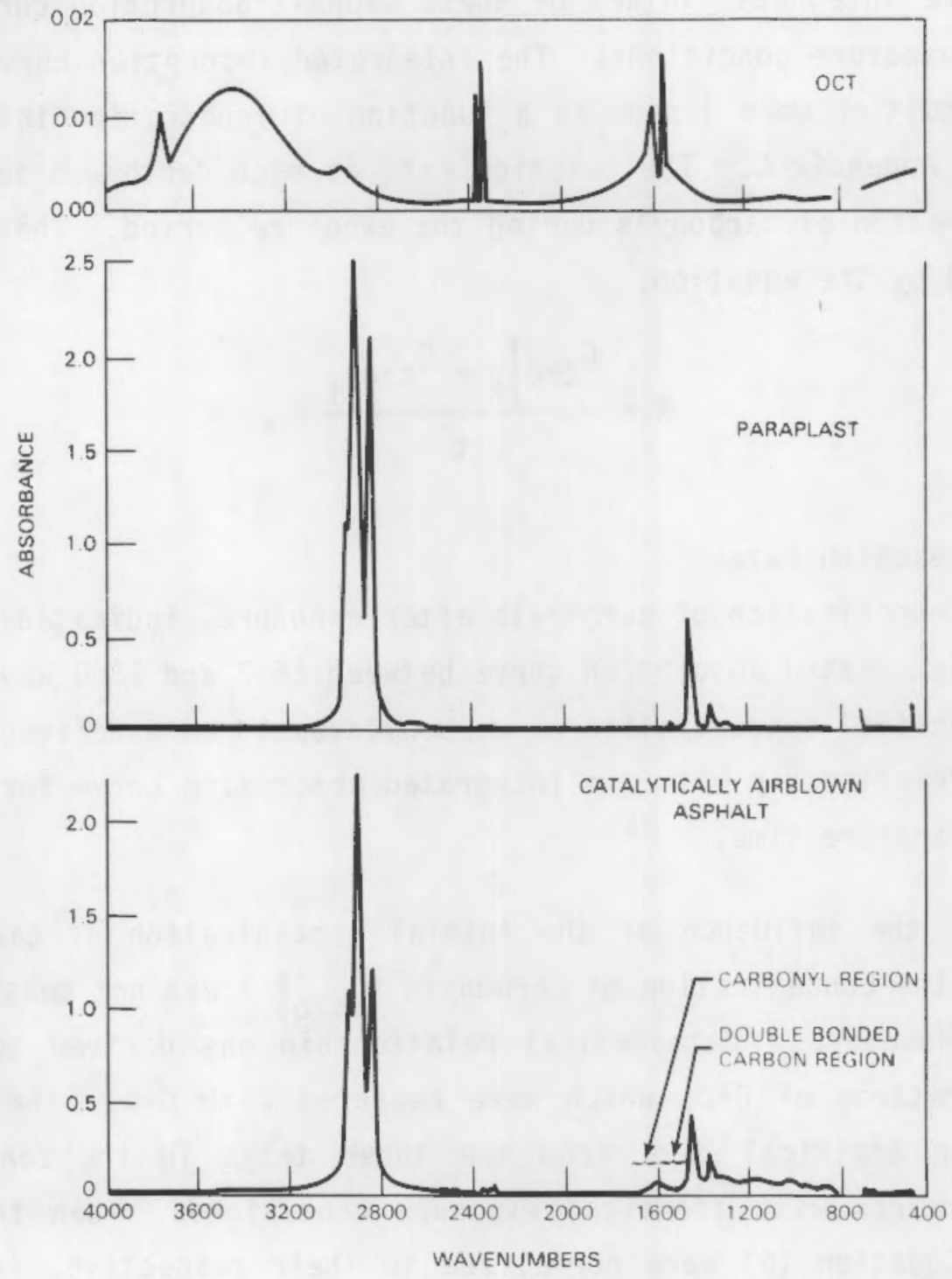

FIGURE 18. Absorption Bands of O.C.T., Paraplast, and Asphalt

associated contributors for each of these materials. The carbonyl absorption band falls within the 1667 to 1800 wavenumber range, and the peak at 1600 wavenumbers is associated with $C=C$ bonds from which carbonyls form.

Reaction rates for each sample were determined in the following manner. First, the contributions of the Paraplast or O.C.T. to the absorption 
spectra were subtracted from the absorption band to obtain the true curve for the asphalt sample. Then, the aging reaction rates were determined by comparing the integrated values of these asphalt absorption curves related to various exposure conditions. The integrated absorption curves for each exposure condition were listed as a function of penetration into the liner as shown in Appendix $C$. The reaction rate at each depth was determined by rate of formation of carbonyls during the exposure period. The process can be expressed by the equation:

$$
R=\frac{\left.{ }^{C_{c=0} \mid}\right|_{f}-\left.{ }^{C_{c=0} \mid}\right|_{i}}{t}
$$

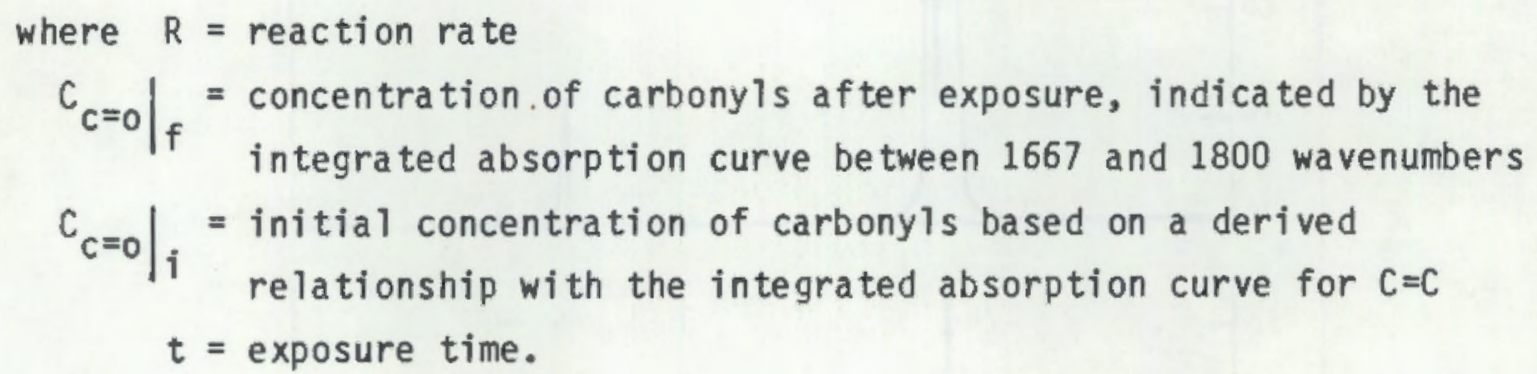

Because the influence of the initial concentration of carbonyls was unexpected, the concentration of carbonyls $\left(\left.C_{c=0}\right|_{i}\right)$ was not measured before exposure. However, a mathematical relationship was derived to correlate the concentrations of $\mathrm{C}=\mathrm{C}$, which were measured with $\mathrm{C}=0$. The expression was based on empirical data from the three tests in the center of the fractional matrix with identical exposure conditions. Then the reaction rates from Equation (6) were normalized to their respective, initial concentrations of carbonyls and plotted for the various exposure conditions as shown in Figures 19, 20, 21, and 22. Examination of these plots revealed some significant findings:

- temperature and oxygen are effective acceleration parameters

- acidity levels have no measurable effect on aging reactions

- no measurable reaction rate is observed under normal, nonaccelerated conditions 


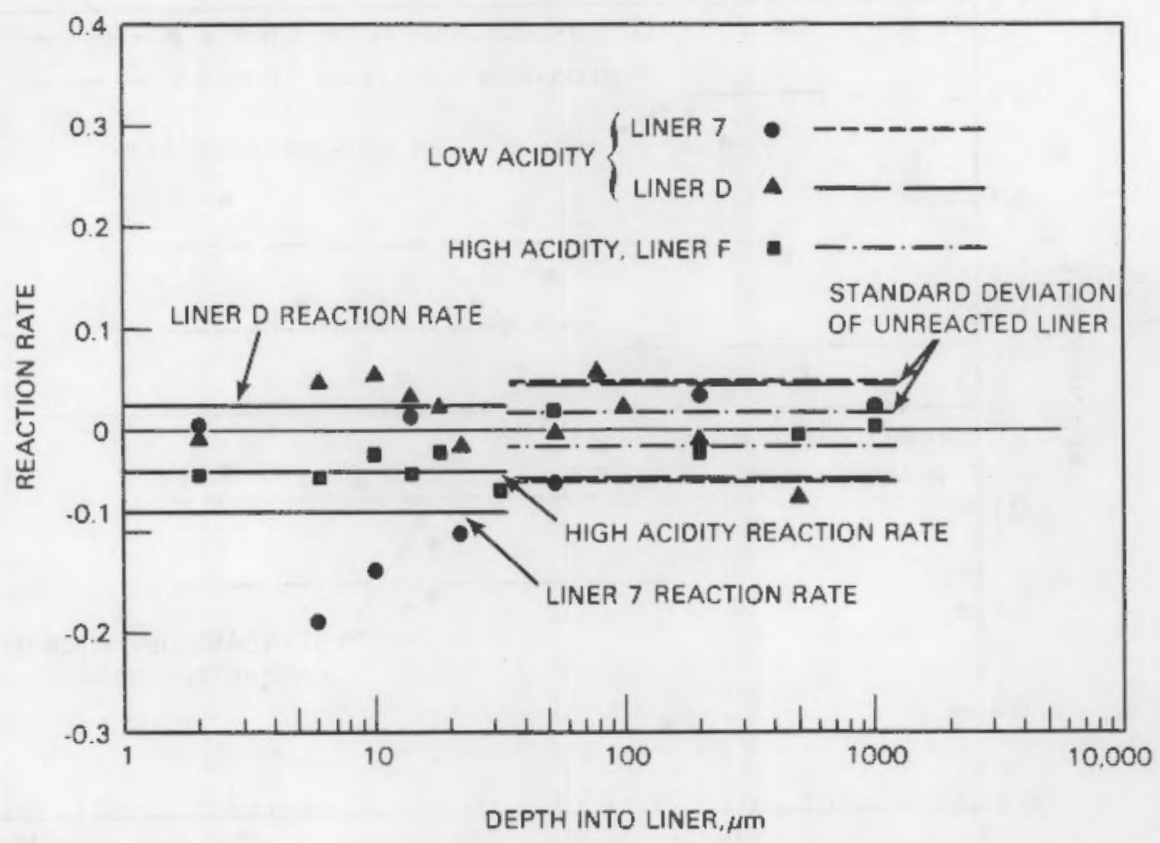

FIGURE 19. Reaction Rates at Normal and Highly Acidic Conditions. Liner designations correspond to data compiled in Appendix C.

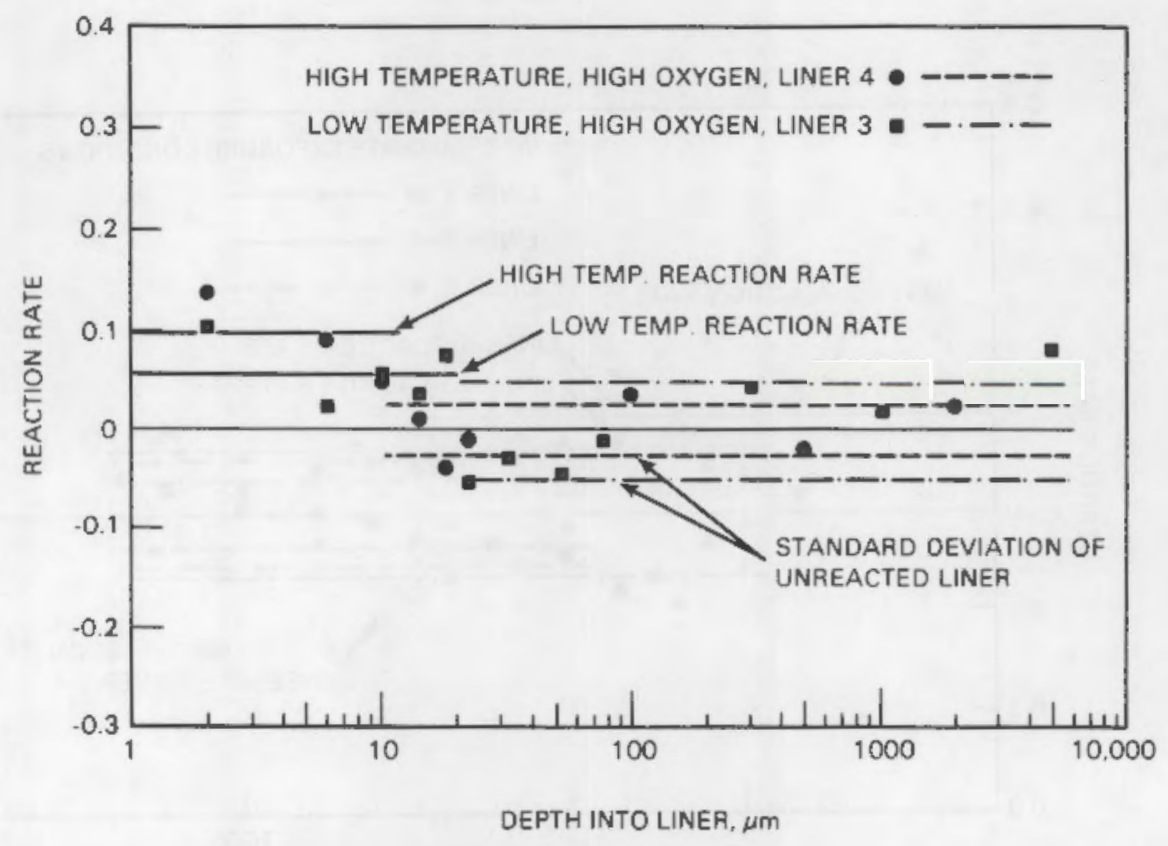

FIGURE 20. Effect of Temperature on Reaction Rates at High Oxygen Concentrations. Liner designations correspond to data compiled in Appendix C. 


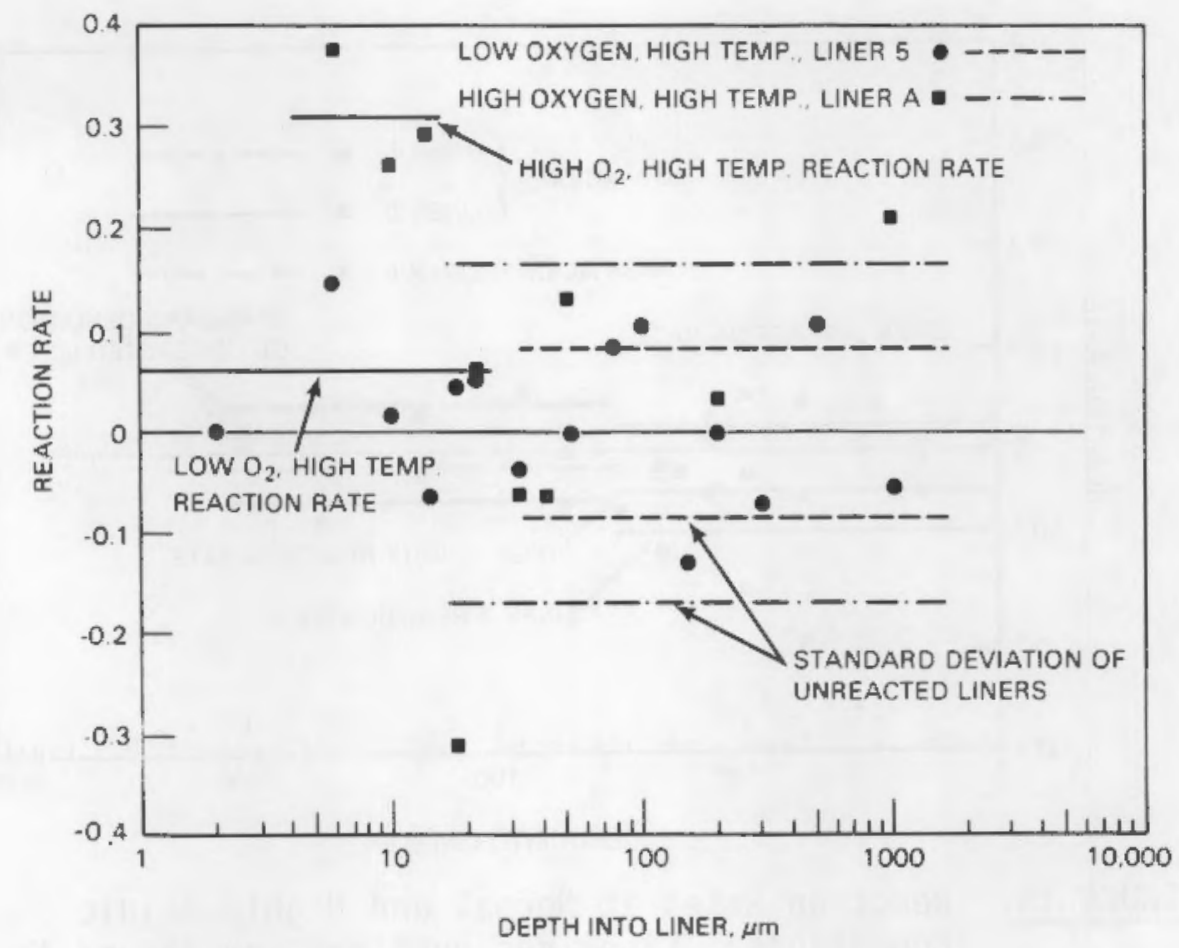

FIGURE 21. Effect of 0xygen on Reaction Rates. Liner designations correspond to data compiled in Appendix C.

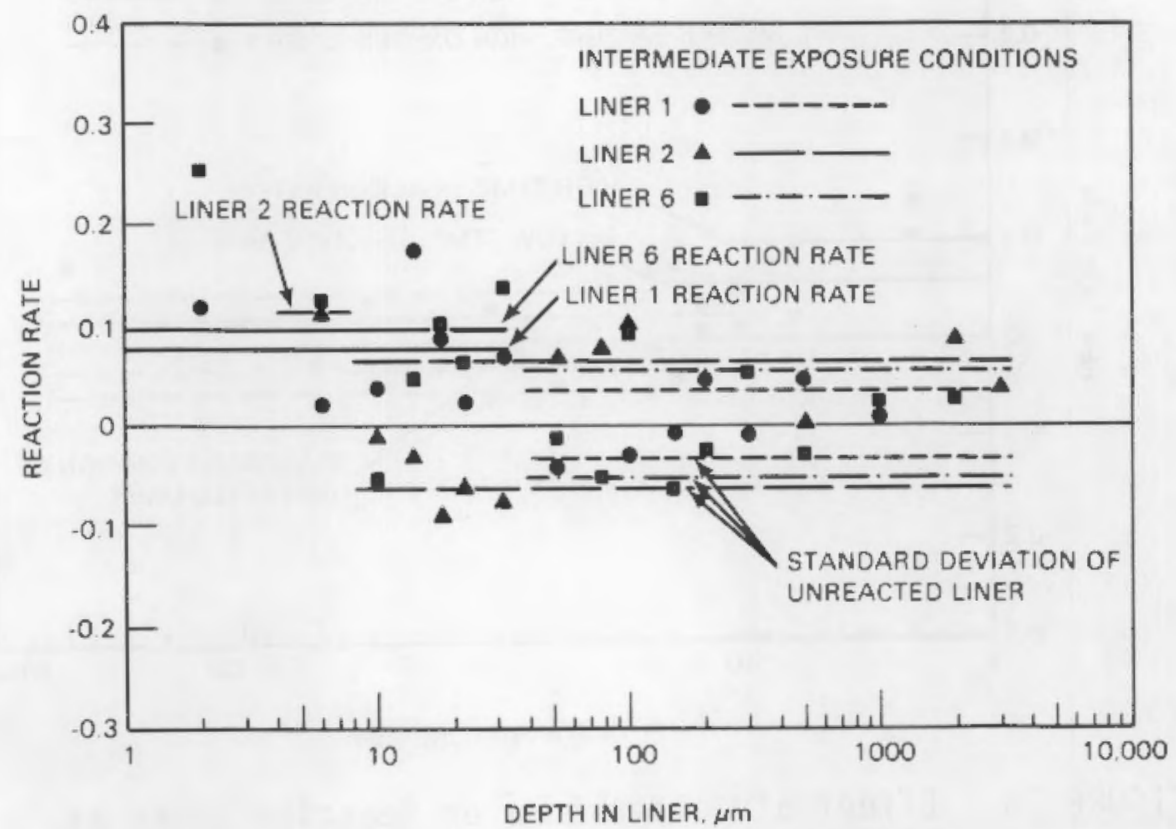

FIGURE 22. Reaction Rates at Intermediate Exposure Conditions. Liner designations correspond to data compiled in Appendix $C$. 
- aging is extremely slow for asphalt, even under accelerated conditions

- aging reactions under accelerated conditions penetrate no deeper than 10 to $40 \mu \mathrm{m}$.

The basis for these conclusions is discussed below. The solid horizontal lines shown in Figures 19 through 22 are arithmetic mean values of the reaction rates measured at the near surface $(10-40 \mu \mathrm{m})$. The standard deviation of the reaction rates beyond $40 \mu \mathrm{m}$ were determined separately to define the bounds of the data scatter. The reaction rate near the surface was considered measurable if the mean value near the surface fell near or outside the standard deviation at greater depths.

Figure 19 illustrates two cases conducted at normal exposure conditions and one at high acidity and low temperature and oxygen conditions. No measurable reaction rates are discernible as a function of depth; therefore, we can conclude that aging reactions are not measurable under normal conditions during the 3-month exposure period. In addition, increased acidity in the leachate did not accelerate the aging reactions. Apparently the oxidizing nature of sulfuric acid is not a significant degradation mechanism for asphalts.

Figure 20 shows the effect of temperature on reaction rates for the same oxygen concentrations. The ratio of reaction rates for these two cases was $0.099 / 0.058=1.7$. A comparison of this low-temperature, highoxygen reaction rate with the high-temperature, high-oxygen rate shown in Figure 21 results in an increased reaction rate near the surface by a factor of 0.31 (high-temperature, high-oxygen, Figure 21)/0.058 (10wtemperature, high-oxygen, Figure 20$)=5.3$. Using the higher ratio of reaction rates, the activation energy in Equation (4) can be calculated to be $15 \mathrm{kcal} / \mathrm{g}-m o l e$, which means that for a temperature increase of $10^{\circ} \mathrm{C}$, the reaction rate increases by a factor of 2.3. This is consistent with Blokker and van Hoorn's findings in their accelerated aging tests with pavement asphalts. 
Figure 21 also shows the effect of oxygen concentration under constant temperature conditions. This comparison indicates that oxygen evidently has a significant effect on aging. From this plot, the value of $n$ in the reaction rate Equation (3) is calculated to be 0.8 . Blokker and van Hoorn determined $n$ to be 0.6 , so this finding was substantiated.

The penetration of aging reactions was limited to a depth of 10-40 $\mu \mathrm{m}$ in the surface of the 7000- $\mu \mathrm{m}$ thick liner. Blokker and van Hoorn reported that penetration of oxygen as deep as $3000 \mu \mathrm{m} \mathrm{might} \mathrm{be} \mathrm{expected} \mathrm{for} \mathrm{thick}$ samples. The apparent discrepancy may be due to the fact that the asphalt liner aging reactions were obtained under saturated leachate conditions, whereas Blokker and van Hoorn's studies were conducted in air.

Figure 22 shows the reaction rates at intermediate exposure conditions. These plots substantiate the quantitative effect of temperature and oxygen on reaction rate. The depth of penetration, 10-40 $\mathrm{m}$, was also substantiated by these measurements. Even though the data scatter was significant, the mean values agreed closely for all three exposed liners. The intermediate exposure results provide an important validation of the accelerated aging methodology and analysis.

\section{Performance Lifetime}

The calculated activation energy $\left(E_{A}\right)$ and dependence of oxygen concentration on reaction rate $(n)$ in the reaction rate equation determined the equivalent exposure period for the maximally exposed liner sample. With a temperature $20^{\circ} \mathrm{C}$ above ambient and $1.7 \mathrm{~atm}$ oxygen partial pressure, the reaction rate was increased by a factor of 30 . In other words, the equivalent aging period of the liner over 3 months at these conditions was 7 years. Because acidity did not act as an accelerating aging parameter and only $40^{\circ} \mathrm{C}$ was achieved at the accelerated temperature (see Appendix $\mathrm{C}$ ), the equivalent exposure period fell short of the desired objective. However, the expected performance lifetime can be extrapolated with some accuracy because the penetration of aging reactions was minimal. 
At maximum exposure conditions, penetration of the reaction products was only $\sim 14 \mu \mathrm{m}--$ or about $0.2 \%$ of the total liner thickness. Maximum penetration was $40 \mu \mathrm{m}$. At the accelerated reaction rate, the time required to penetrate the entire depth of the liner might be increased by a factor of 200 to 500. Based on the testing that represented seven years of aging, the asphalt membrane performance could be expected to greatly exceed the 1000-year standard. If needed to prevent groundwater contamination, catalytically airblown asphalt could, therefore, be expected to perform extremely well as a leachate barrier at inactive uranium mill tailings piles for long periods.

\section{CONTROLLED CLAY LINER EVALUATIONS}

Although the sodium bentonite amended to natural soil proved to be the most effective leachate barrier of the clay liner category, natural soils without amendments were considered to have adequate retardation capabilities for most inactive mill tailings sites. Therefore, in addition to conducting further studies with sodium bentonite amendments, two calcareous soils from $\mathrm{Clive,} \mathrm{Utah,} \mathrm{were} \mathrm{evaluated.}$

These evaluations were conducted not only to determine permeability but also to assess the sorptive and coprecipitative properties of the clay and bentonite amended soils. After material characterizations, the soils and clay liner were interacted with acidic and alkaline leachates; and batch and column interaction studies were performed. The batch studies indicated overall retardation capabilities, such as sorption and buffering, while the column studies determined permeability and rate of contaminant break through.

\section{Material Characterization}

The bentonite liner material (soil plus $10 \%$ sodium bentonite) and two soil samples from Clive, Utah (Soil \#2 and Soil \#6) were characterized (see Table 13). The soils from Clive had a very high calcium carbonate $\left(\mathrm{CaCO}_{3}\right)$ content, and an unusually high level of excess salts (compared with normal 
TABLE 13. Characteristics of Clive, Utah, Soil Samples

Depth

$\frac{\text { Soil \#6 }}{30-61 \mathrm{~cm}} \quad \frac{\begin{array}{l}\text { Liner Material } \\ \text { (Soil plus 10\% } \\ \text { Na-Bentonite) }\end{array}}{30-61 \mathrm{~cm}} \quad \frac{\text { Soil \#2 }}{180 \mathrm{~cm}}$

Particle Size

\% Sand

17

15

13

\% silt

46

42

31

\& Clay

36

42

55

\% Organic Matter

0.91

0.93

0.66

Carbonate Content

$\% \mathrm{CaCO}_{3}$

27

25

57

Cation Exchange Capacity

(meq/100 g)

18

24

15

Surface Area $\left(\mathrm{m}^{2} / \mathrm{g}\right)$

$77 \pm 15$

$131 \pm 8$

$62 \pm 13$

Saturated Paste

$\mathrm{pH}$

8.3

8.4

8.6

E.C. (mmhos $/ \mathrm{cm}$ )

48

30

14

Buffering Capacity (a)

$\mathrm{HCl}$ equivalent (meq/100 g) 450

410

1100

(a) Amount of total acidity required to lower the soil pH to 4.5 .

agricultural soils) was indicated by the high electrical conductivity measurements made on saturated soil pastes. Ionic strengths were calculated using the method described by Lindsay (1979). Saturated paste E.C. measurements indicated that ionic strength ranged from 0.2 to $>0.7 \mathrm{moles} / \mathrm{L}$.

To determine buffering capacities, $1 \mathrm{~g}$ of soil in $50 \mathrm{~cm}^{3}$ of distilled water was titrated with $\mathrm{HCl}$. During titration, the $\mathrm{pH}$ dropped very slowly between pH 8 and pH 7, but more quickly between pH 7 and pH 5 . Below pH 5, however, the value dropped rapidly to $<2.0$. 
The mineralogical compositions of these soils and liner material were analyzed by X-ray diffraction on random-packed powder mounts and oriented slurries of each bulk sample. Based on the relative intensities of certain reflections, semiquantitative estimates were made of the relative abundance of mineral phases.

Both soils and the liner material (soil plus $10 \%$ sodium bentonite) contained quartz, carbonates (calcite, dolomite and aragonite), kaolinite, illite and feldspar. The carbonates and quartz were the major phases, and the clay minerals (kaolinite and illite) were minor phases. Soil \#2 contained the least feldspar and the most aragonite.

\section{Leachate Interactions with Soil and Liner Materials}

Batch and column methods were both used to study the interactions of tailings leachates with the two soils and the liner material. Synthetic leachates were prepared to represent the initial 0.20 to 0.30 pore volumes of effluent from the tailings column leach tests (see Table 14). For the

batch tests, $250 \mathrm{~cm}^{3}$ of synthetic tailings leachate were shaken with $50 \mathrm{~g}$ of soil or liner material in a 1-L polyethylene bottle (similar to the 5:1 batch tailings leach tests).

The soils and liner material were titrated with synthetic leachate, the same procedure used to measure the buffering capacity except that synthetic leachate was substituted for $\mathrm{HCl}$.

Permeability values (saturated hydraulic conductivities) were measured using columns that were $8.9 \mathrm{~cm}$ long and $8.9 \mathrm{~cm}$ in diameter. Synthetic leachate was percolated upward through each column and collected in separate covered graduated cylinders. Pressure was maintained on the leachate using compressed air in the leachate reservoir.

Titration experiments and batch contact of synthetic leachate with the soils and liner material have shown that these earthen materials are very effective at neutralizing acidic leachates but not the alkaline Ourango leachate. 
TABLE 14. Comparison of Synthetic Leachate Compositions Before and After Contact with Soil \#6 in 5:1 Solution to Solid Ratio Batch Test (concentrations are in $\mathrm{mg} / \mathrm{L}$ )

\begin{tabular}{|c|c|c|c|c|c|c|}
\hline & \multicolumn{2}{|c|}{ Shiprock } & \multicolumn{2}{|c|}{ Salt Lake } & \multicolumn{2}{|c|}{ Durango } \\
\hline & Before & After & Before & After & Before & After \\
\hline Al & 2860 & 0.27 & 1230 & 0.12 & 5.3 & 0.24 \\
\hline $\mathrm{Ca}$ & 521 & 420 & 1.1 & 500 & 5.6 & 3.0 \\
\hline $\mathrm{Cu}$ & 130 & 0.06 & 47 & 0.02 & ND & 0.02 \\
\hline $\mathrm{Fe}$ & 177 & 0.09 & 1800 & 0.04 & 0.04 & 0.01 \\
\hline K & 61 & 160 & ND & 21 & 44 & 72 \\
\hline $\mathrm{Mg}$ & 2060 & 2000 & 165 & 250 & 16 & 10 \\
\hline Mn & 67 & 19 & 56 & 0.4 & 0.16 & 0.07 \\
\hline $\mathrm{Na}$ & 1560 & 2600 & 119 & 1200 & 2080 & 2500 \\
\hline $\mathrm{Ni}$ & 11 & $N D^{(a)}$ & 22 & ND & ND & ND \\
\hline $\mathrm{Sr}$ & 1.9 & 3.3 & ND & 7.1 & 0.06 & 0.06 \\
\hline $\mathrm{Zn}$ & 112 & 0.2 & 86 & 0.1 & ND & 0.1 \\
\hline B & ND & 4.7 & ND & 4.9 & 0.02 & 3.2 \\
\hline $\mathrm{Cl}$ & 874 & 3600 & 1110 & 1300 & 26 & 870 \\
\hline $\mathrm{CO}_{3}$ & ND & 600 & ND & 200 & 1800 & 1800 \\
\hline$F$ & 16 & 9.7 & 3.5 & NO & 17 & 1.8 \\
\hline Mo & ND & 0.2 & ND & 0.03 & 0.8 & 0.1 \\
\hline $\mathrm{NO}_{3}+\mathrm{NO}_{2}$ & 870 & 130 & 307 & ND & 74 & 60 \\
\hline $\mathrm{PO}_{4}$ & 14 & ND & 6 & ND & 76 & 45 \\
\hline $\mathrm{SO}_{4}$ & 30,000 & 8500 & 12,500 & 2600 & 1370 & 290 \\
\hline $\mathrm{Si}$ & 11.5 & 2.2 & ND & 1.5 & 2.1 & 4.5 \\
\hline As (ppm) & 3.8 & 0.2 & 0.1 & $\leqslant 0.1$ & 1.2 & 0.7 \\
\hline $\mathrm{Ba}$ & ND & 0.01 & ND & 0.09 & 0.1 & 0.02 \\
\hline Cd & 4.7 & 0.05 & 2.5 & $\leqslant 0.01$ & 0.01 & $\leqslant 0.01$ \\
\hline $\mathrm{Cr}$ & 1.9 & $\leqslant 0.02$ & 2.3 & $\leqslant 0.02$ & 0.07 & 0.04 \\
\hline $\mathrm{Pb}^{(\mathrm{b})}$ & 5.0 & 0.2 & 2.4 & 0.2 & ND & ND \\
\hline $\mathrm{pH}$ & 2.1 & 7.7 & 2.1 & 7.7 & 9.7 & 9.4 \\
\hline E.C. $(m \mathrm{mho} / \mathrm{cm})$ & 26 & 20 & 11.0 & 8.2 & 10.0 & 9.5 \\
\hline Percent Sodium $(c)$ & & 38 & & 53 & & 97 \\
\hline $\begin{array}{l}\text { Total Cations } \\
(\text { meq } / \mathrm{L})(\mathrm{d})\end{array}$ & 600 & 300 & 227 & 98 & 94 & 112 \\
\hline $\begin{array}{l}\text { (a) } \mathrm{ND}=\text { Not detec } \\
\text { (b) } 5 \mathrm{ppm} \mathrm{Pb} \text { added } \\
\text { (c) Percent sodium } \\
\text { (d) Total cations }\end{array}$ & $\begin{array}{l}\text { the sy } \\
100 \times[ \\
\text { he sum }\end{array}$ & $\begin{array}{l}\text { netic } 1 \\
\text { /( Tota } \\
\text { al } 1 \text { ca }\end{array}$ & $\begin{array}{l}\text { tions) } \\
\text { s in m }\end{array}$ & $\begin{array}{l}\text { leq/L. } \\
1 / L\end{array}$ & & \\
\hline
\end{tabular}


TABLE 15. Buffering Capacities of Clive, Utah, Soils and Liner Material

Liner Material

(Soil plus $10 \%$

Soil \#6 Na-bentonite)

25

500

410

450

470
1100

Soil \#2

1250

(a) Amount of acid ( $\mathrm{HCl}$ ) required to lower the soil $\mathrm{pH}$ to 4.5 .

(b) Total acidity (as leachate) required to lower the soil pH to 4.5 .

The soils described in Table 13 were titrated with synthetic Shiprock tailings leachate for comparisons with the buffering capacities measured by $\mathrm{HCl}$ titration and those calculated from $\mathrm{CaCO}_{3}$ contents. Total acidity for the Shiprock leachate was calculated using the sum of $\mathrm{Al}^{+3}, \mathrm{Fe}^{+3}$ and $\mathrm{H}^{+}$ concentrations in milliequivalents per liter. The buffering capacity measurements (to $\mathrm{pH} 4.5$ ) listed in Table 15 are very close to the $\mathrm{CaCO}_{3}$ contents in these soils.

Below $\mathrm{pH} 4.5$, a significant difference was observed between the titration curves produced by Shiprock leachate and $\mathrm{HCl}$. The $\mathrm{HCl}$ titration indicates the buffering capacity of the soil carbonates. The $\mathrm{pH}$ dropped slowly to 4.5; then, addition of more $\mathrm{HCl}$ caused a rapid decline in $\mathrm{pH}$ to less than 2.0. Figure 23 shows a very large buffering capacity below $\mathrm{pH}$ 4.5 when Shiprock leachate was used as the titrant but not when $\mathrm{HCl}$ was used.

Titration of distilled water with Shiprock leachate produced a curve similar to the soil-leachate curve when the $\mathrm{pH}$ dropped below $\mathrm{pH} 4.5$. A 


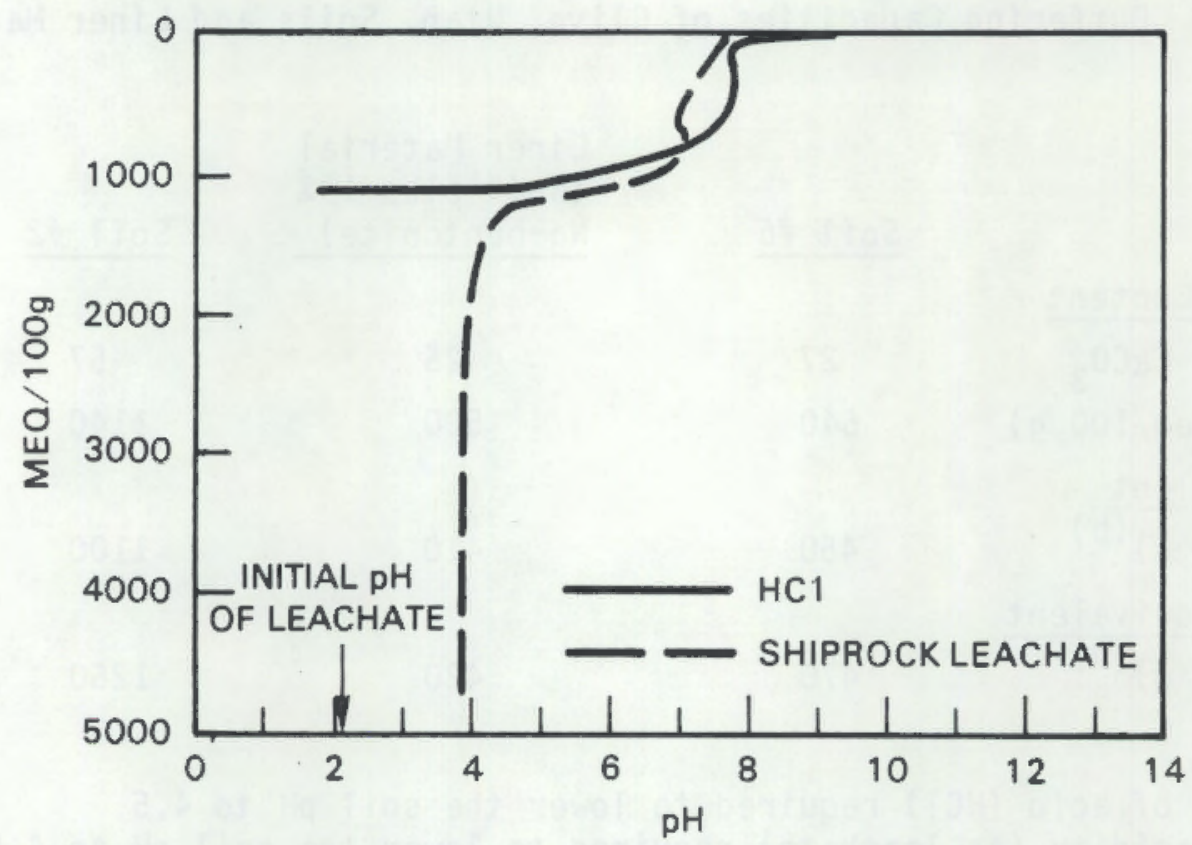

FIGURE 23. Titration Curves for Soil \#2 Using $\mathrm{HCl}$ and Shiprock Tailings Leachate (dashed line)

cloudy suspension resulting from the blank titration also indicated that a precipitation reaction had occurred. Because of the relatively high solubility of $\mathrm{Fe}$ under the conditions of the blank titration, it is likely that $\mathrm{Al}^{+3}$ precipitated. Dissolution of the Al precipitate below $\mathrm{pH} 4.5$ seems to be the most likely mechanism for the "extra buffering capacity" of this soil for Shiprock tailings leachate (see discussion below).

The information presented above indicates that soils from the clive, Utah, site have a very high buffering capacity for acidic tailings leachate. The total acidity of tailings leachate equals the sum of the concentrations of $\mathrm{H}^{+}, \mathrm{Al}^{+3}$ and $\mathrm{Fe}^{+3}$ (meq/L). For the Shiprock tailings leachate, more than $95 \%$ of the total acidity was caused by hydrolysis of $\mathrm{Al}^{+3}$ as the leachate $\mathrm{pH}$ increased on contact with the soil. Several Al compounds such as gibbsite and alunite may also precipitate but only amorphous $\mathrm{Al}(\mathrm{OH})_{3}$ will be considered for simplicity. The hydrolysis of $\mathrm{Al}^{+3}$ can be expressed as

$$
\mathrm{Al}^{+3}+3 \mathrm{H}_{2} \mathrm{O}=\mathrm{Al}(\mathrm{OH})_{3}+3 \mathrm{H}^{+}
$$


As $\mathrm{Al}^{+3}$ hydrolyzes, it releases $\mathrm{H}^{+}$to solution which can react with solid carbonate phases that neutralize the acidity

$$
2 \mathrm{H}^{+}+\mathrm{CaCO}_{3}=\mathrm{Ca}^{+2}+\mathrm{CO}_{2}(\mathrm{~g})+\mathrm{H}_{2} \mathrm{O}
$$

As the $\mathrm{CaCO}_{3}$ dissolves and releases $\mathrm{Ca}^{2+}$, the high sulfate concentrations present in the leachate cause precipitation of gypsum:

$$
\mathrm{Ca}^{2+}+\mathrm{SO}_{4}{ }^{2-}=\mathrm{CaSO}_{4} \cdot 2 \mathrm{H}_{2} \mathrm{O} \text { (gypsum) }
$$

When the $\mathrm{pH}$ drops to about 4.5, the $\mathrm{pH}$ buffering capacity of the carbonates is exhausted; however, Al compounds may buffer the $\mathrm{pH}$ through the reversal of the $A]$ precipitation reaction

$$
\mathrm{Al}(\mathrm{OH})_{3}+3 \mathrm{H}^{+}=\mathrm{AT}+3+3 \mathrm{H}_{2} \mathrm{O}
$$

As the Al compounds dissolve, $\mathrm{H}^{+}$is consumed and the $\mathrm{pH}$ is buffered below 4.5 as shown in Figure 23.

These reactions have three effects: 1) neutralization of leachate acidity to $\mathrm{pH} 4.5$ [see Figure 23 (equivalent to the $50 i 1 \mathrm{CaCO}_{3}$ content)]; 2) increase in the solid-phase volume because of the lower specific gravity of gypsum $\left(2.3 \mathrm{~g} / \mathrm{cm}^{3}\right)$ compared to soil carbonates $\left(2.7\right.$ to $\left.3.0 \mathrm{~g} / \mathrm{cm}^{3}\right)$; and 3) precipitation of $\mathrm{Fe}$ and $\mathrm{Al}$ compounds that may buffer the leachate $\mathrm{pH}$ be low $\mathrm{pH} 4.5$.

The precipitation of gypsum, aluminum and iron compounds and precipitates of other cations from the neutralized leachate will reduce contaminant levels through adsorption/coprecipitation reactions and reduce the porosity and permeability of the soil or liner material.

Analysis of the end point ( $\mathrm{pH} 3.8$ ) solution resulting from the soil titration with synthetic Shiprock leachate showed that $\mathrm{Cd}, \mathrm{Ce}, \mathrm{Li}, \mathrm{Mg}, \mathrm{Mn}$, $\mathrm{Na}$, and $\mathrm{Zn}$ concentrations decreased by 50-70\%, while $\mathrm{Al}, \mathrm{As}, \mathrm{Cr}, \mathrm{Cu}, \mathrm{Fe}$, and $\mathrm{SO}_{4}$ concentrations decreased by $>70 \%$ compared to the initial leachate 
concentrations. Dilution of the leachate during titration would account for only a 33\% decrease in concentrations. The rest of the concentration decreases were caused by precipitation, coprecipitation, and adsorption reactions.

Table 14 lists chemical compositions of the three leachates after contacting Soil \#6. (These results are similar to the leachate compositions after contact with the other two soils). Precipitation caused total cation (and anion) concentrations to decrease in both acidic leachates.

Analysis of soil mineralogy by X-ray diffraction after contacting acidic leachate from Salt Lake and Shiprock tailings indicated that 1) soil carbonates (calcite, dolomite, and aragonite) decreased in abundance, and 2) gypsum formed in the soils. Gypsum was not detected in soils contacting alkaline Durango leachate (although sulfate concentrations decreased significantly in the leachate), and the soil carbonate abundances were apparently unchanged by contact with Durango leachate.

Permeability values for the liner material (soil plus $10 \%$ sodium bentonite) decreased with time as leachate passed through the columns. Sodium-saturated bentonite clay is known to swell and disperse when contacted with low ionic strength solutions having a high percentage of dissolved sodium (Sherard, Dunnigan and Decker 1976; Cass and Sumner 1982). At high ionic strengths, the clay may remain flocculated even when the solution has a high sodium percentage.

The soils and liner studied contained a considerable amount of excess sodium salts, which appeared to prevent dispersion and swelling of the clay upon initial contact between leachate and liner. The liner permeability generally decreased as the effluent electrical conductivity (therefore ionic strength) decreased. This is a good indication that the sodium bentonite became dispersed and swelled as the excess sodium salts were washed from the column. The dispersed and swollen clay particles plugged up conducting pores in the liner and reduced the permeability. 


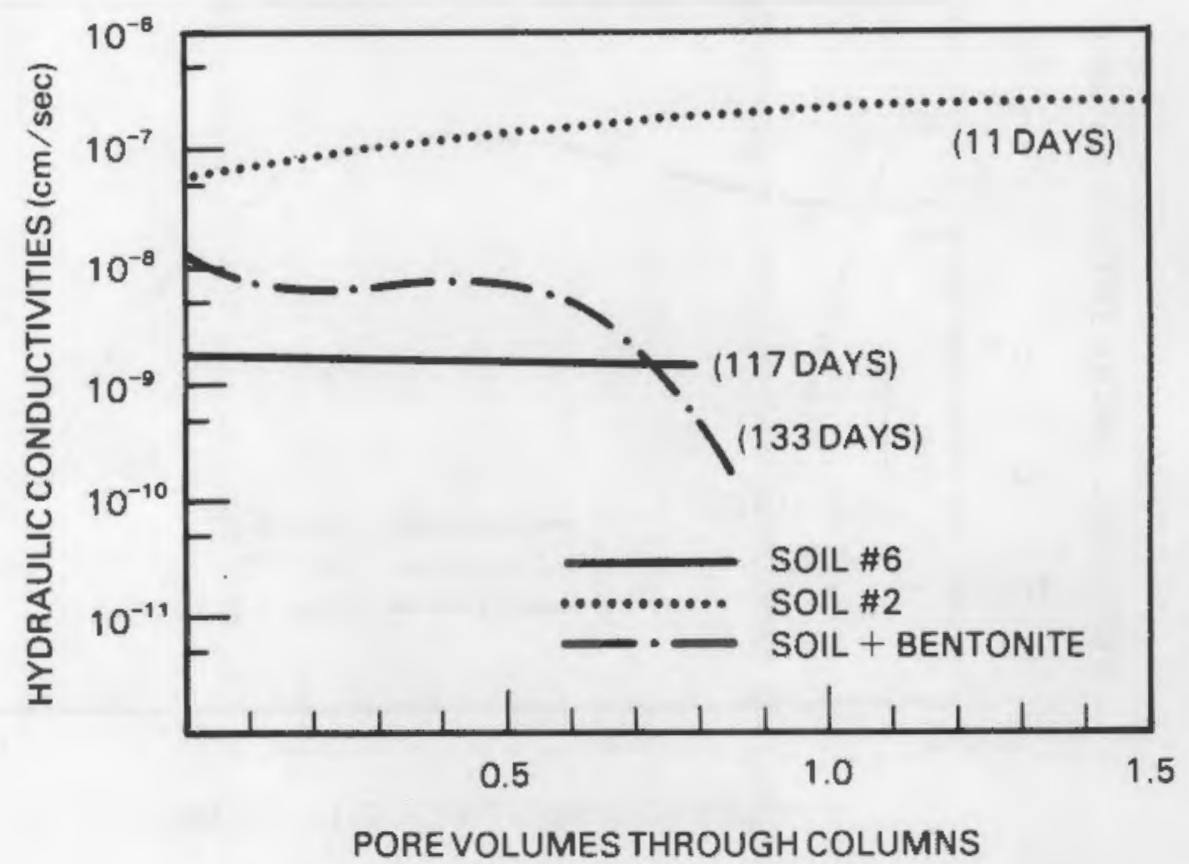

FIGURE 24. Sa tura ted Hydraulic Conductivity $\mathrm{K}, \mathrm{cm} / \mathrm{s}$, as a Function of Durango Tailings Leachate Pore Volumes Passing Through the Soil Columns

Permeability values for Durango leachate (see Figure 24) contacting the two soils without bentonite tended to remain constant with time even though the electrical conductivity (ionic strength) decreases; however, permeability of the bentonite-amended soil decreased. The Durango leachate had a low ionic strength (compared to the two acid leachates) and a sodium percentage of $96 \%$, which would disperse the bentonite, according to Sherard et al. (1976). The constant permeability values for the other two soils that contacted Durango leachate support the argument that dispersion and swelling of the clay contributed to the decrease in permeability observed for the liner material.

For the alkaline Durango leachate, clay swelling and dispersion were probably the major mechanisms for a decrease in permeability. For the two acidic leachates, however, precipitation of gypsum and other compounds may also contribute to observed decreases in permeability. 


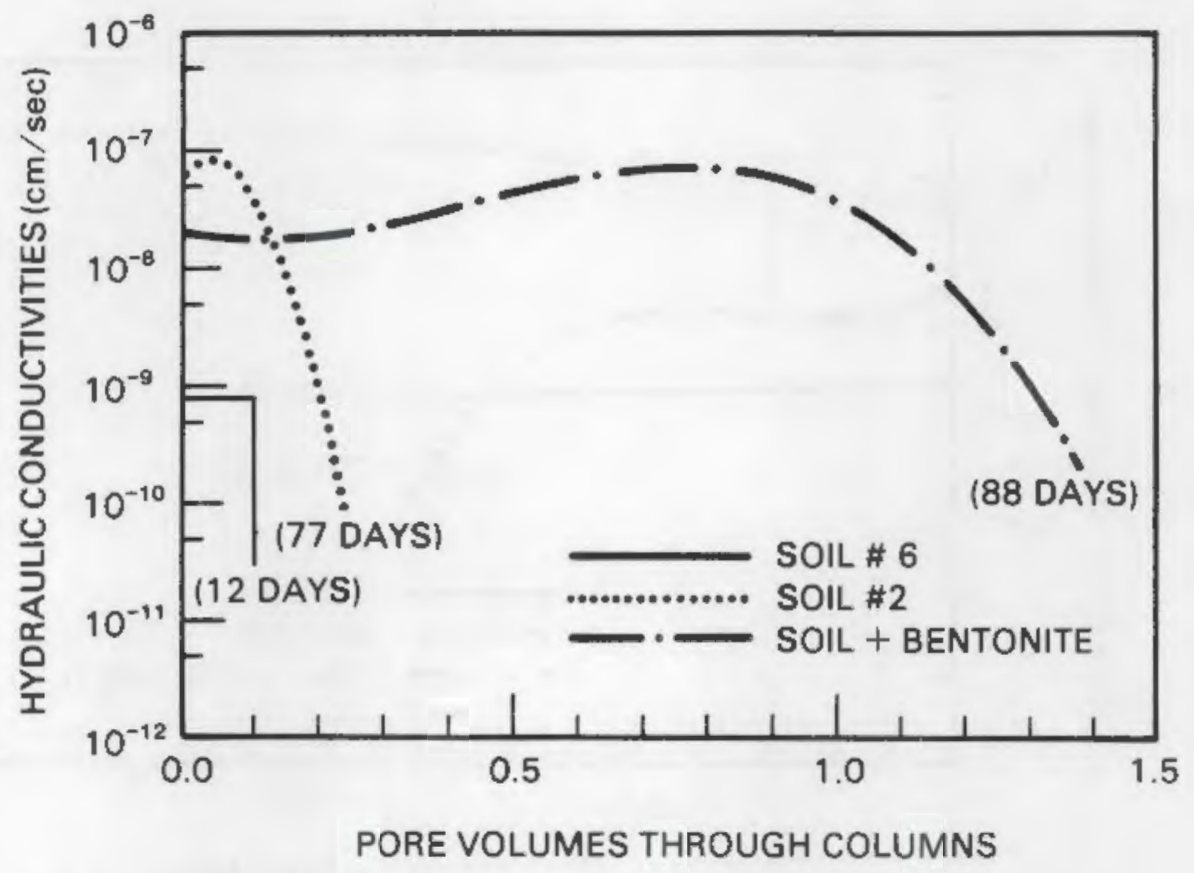

FIGURE 25. Sa turated Hydraulic Conductivity $\mathrm{K}, \mathrm{cm} / \mathrm{s}$, as a Function of Shiprock Tailings Leachate Pore Volume Passing Through the Soil Columns

Experimentally observed permeability values decreased to about $10^{-10}$ $\mathrm{cm} / \mathrm{s}$ or less for all soil or liner columns contacting the high sulfate (34 grams of sulfate per liter) Shiprock leachate (Figure 25). Permeability values for the lower sulfate $(13 \mathrm{~g} / \mathrm{L})$ Salt Lake leachate contacting the high carbonate soil (55\% $\mathrm{CaCO}_{3}$ equivalent) and the liner material (25\% $\mathrm{CaCO}_{3}$ wi th $10 \%$ bentonite) both decreased to $<10^{-10} \mathrm{~cm}$ per second. However, the combination of low sulfate leachate (Salt Lake) and low carbonate soil $\left(25 \% \mathrm{CaCO}_{3}\right)$ without bentonite resulted in a relatively constant permeabil$i$ ty of about $10^{-9} \mathrm{~cm} / \mathrm{s}$ (Figure 26 ).

The Salt Lake leachate had less total acidity $(50 \%)$, less dissolved Al (50\%), and less sulfate (40\%) than the Shiprock leachate. Thus, precipitation of gypsum, Al, and Fe compounds from Salt Lake leachate would be not be expected to occur as rapidly (at the same flow rate) as it would from Shiprock leachate. If all $\mathrm{CaCO}_{3}$ in Soil \#6 $\left(25 \% \mathrm{CaCO}_{3}\right)$ dissolves, gypsum, $A 1$, and Fe precipitates will form and a decrease in both porosity and permeability should eventually be observed after more pore volumes of leachate have percolated through the column. 


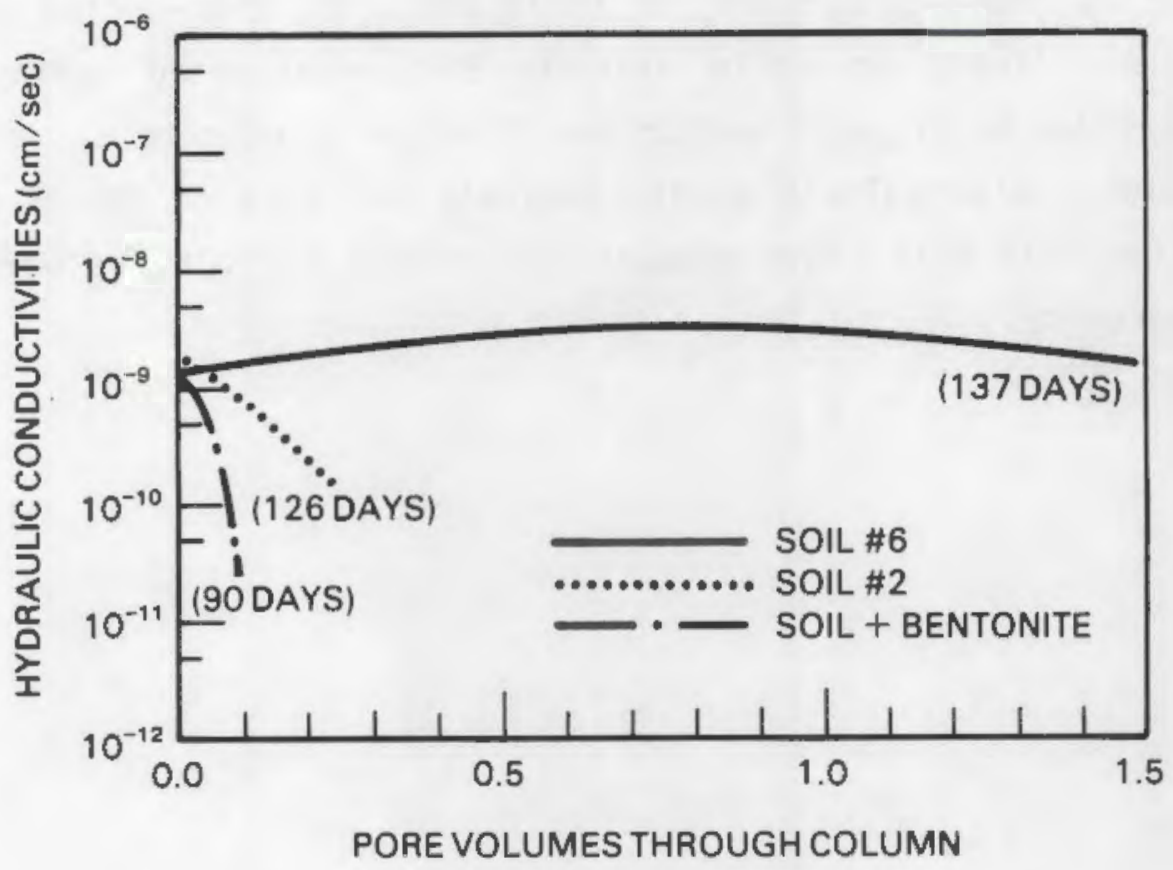

FIGURE 26. Saturated Hydraulic Conductivity $\mathrm{K}, \mathrm{cm} / \mathrm{s}$, as a Function of Salt Lake Leachate Pore Volumes Passing Through the Soil Columns

Characterization of the tailings showed that both Shiprock and Salt Lake tailings leachate were in equilibrium with gypsum to at least two pore volumes. Therefore, gypsum precipitated during neutralization of leachate acidity by soil carbonates should not dissolve on contact with fresh (unneutralized) leachate as long as leachate equilibrium with gypsum is maintained (i.e., high Ca concentrations). Moreover, the permeabilities of these soils or liners will not increase later as a result of acid leachate dissolution of gypsum.

In summary, these studies demonstrated that the $\mathrm{pH}$ buffering capacities of the two Clive, Utah, soils and one liner material for acidic tailings leachate resulted from two reactions: 1) dissolution of soil carbonate phases, and 2) dissolution of Al (and perhaps Fe) precipitates formed during the leachate-carbonate reaction. 
Permeability values will decrease with time under at least two sets of conditions: 1) contact between low ionic strength, high-sodium percentage leachates and liners or soils rich in sodium-saturated bentonite that causes the clay to disperse and/or swell and plug up pore spaces; and 2) contact between high sulfate acidic leachate and soil or liners with high carbonate contents that cause gypsum precipitates and plug up pore spaces, reducing porosity. 


\section{FIELD LINER EVALUATIONS}

The catalytic airblown asphalt membrane and natural soil amended with sodium bentonite were also subjected to field studies beginning in September 1981. The liners were installed in a pit carved out of the Grand Junction tailings pile, instrumented, and covered with tailings, which were subsequently saturated with water. After an exposure of almost 2 years, the materials were sampled and analyzed for performance.

\section{TEST DESCRIPTION}

Figure 27 illustrates the construction of the pit in which the liners were installed. The pit was $29 \mathrm{~m}(96 \mathrm{ft})$ long, $22 \mathrm{~m}(72 \mathrm{ft})$ wide, and 1.8 $m(6 \mathrm{ft})$ deep. The slope of the walls was limited to an approximate $4: 1$ grade. To isolate any extraneous moisture and prevent $i$ ts migration into the foundation soil from the tailings beneath the liners, a moisture barrier consisting of clear rock, 25-35 cm (10-14 in.) thick, was placed on top of the tailings. This provided a capillary moisture barrier so that any moisture accumulated in the foundation soil beneath the liners could be attributed to permeation through the liners.

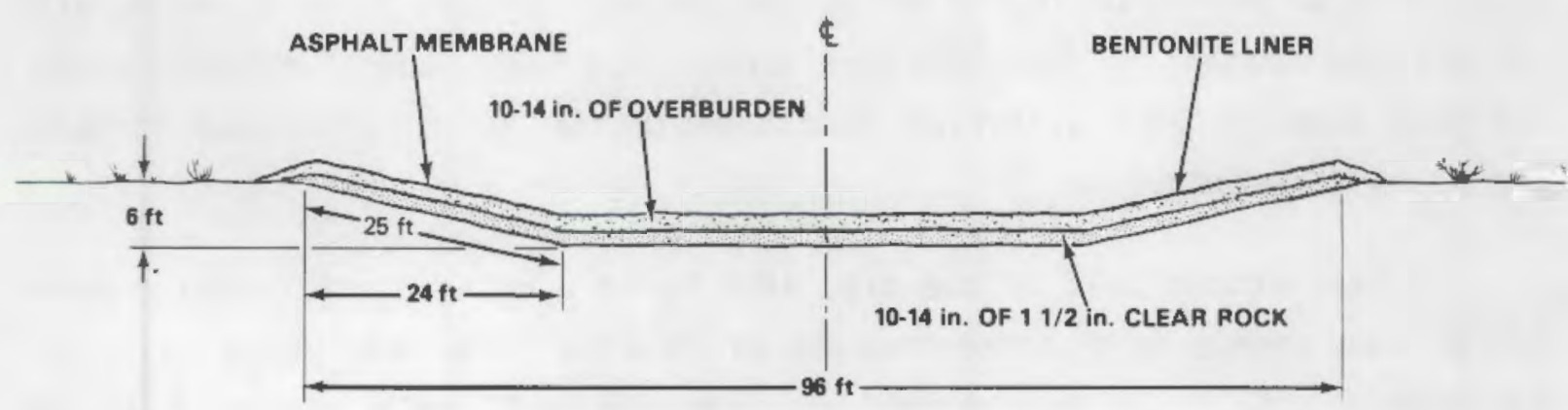

FIGURE 27. Cross Section of Tailings Pit for Liner Evaluation in the Field 
The clear rock layer was covered with a thin sheet of polyethylene membrane to keep the foundation soil from sifting into the voids. The membrane was not installed to prevent moisture migration downward; thus, as portions of the foundation soil became saturated, they could drain through the clear rock. A 25-35 cm (10-14 in.) thick layer of foundation soil (overburden) was installed over the polyethylene membrane.

The foundation soils underlying the asphalt membrane and sodium bentonite liners were separated by the polyethylene membrane at the centerline of the pit. This prevented cross-contamination of leachates that might have permeated either liner and allowed each liner to be evaluated independently.

The foundation soil under each liner was instrumented with an array of moisture blocks and moisture extraction cups (see Figure 28 for locations). The moisture blocks provided readings on the change of saturation profiles in the foundation soil, and the moisture extraction cups provided samples of leachate that passed through the $\operatorname{liner}(s)$ even under unsaturated foundation soil conditions.

The catalytic airblown asphalt membrane (specifications are listed in Appendix B) was sprayed at $200^{\circ} \mathrm{C}$ from a distributor truck over the western half of the pit. Approximately $2600 \mathrm{~kg}$ of catalytic airblown asphalt was sprayed to a thickness of $0.7 \mathrm{~cm}$ in two passes. Figure 29 is a photograph of the application. The moisture extraction cup tubes, through which leachate samples were withdrawn from beneath the liner, protruded through the asphalt membranes.

On the eastern half of the pit, $6800 \mathrm{~kg}$ of Wyoming-quality sodium bentonite was spread at a concentration of $20 \mathrm{~kg} / \mathrm{m}^{2}$ over the $30-\mathrm{cm}$ layer of foundation soil. It was rototilled into the soil to a depth of $10 \mathrm{~cm}$ (shown in Figure 30), moistened to 12-18\% moisture along the walls of the pit, and compacted. Great care was taken to maintain a good seal between the bentonite layer and moisture extraction cup tubes, which protruded through the liner. 


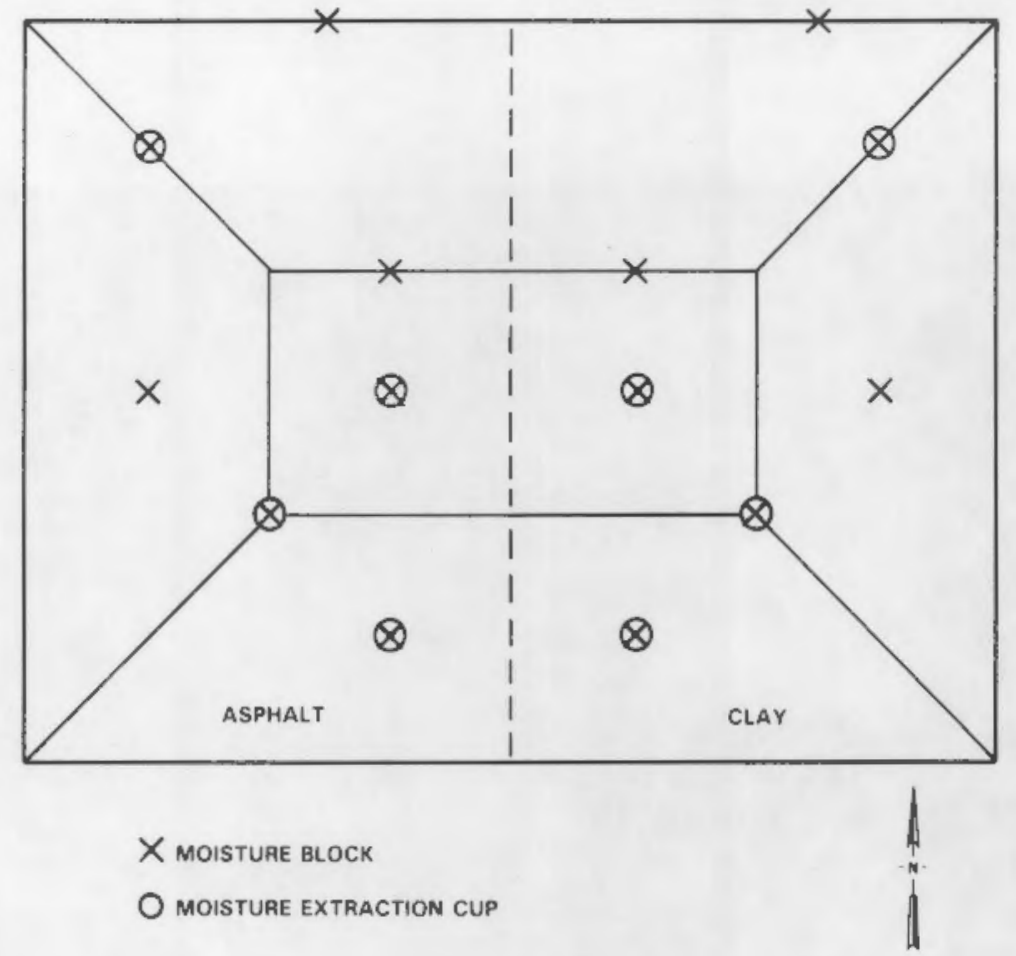

FIGURE 28. Locations of Moisture Blocks and Extraction Cups

Following inspection of the liner installations, tailings were reintroduced into the pit and saturated with fresh water. Piezometers were installed in the tailings at the same locations as the moisture extraction cups to determine the saturated water level. The tailings were covered with a layer of overburden to prevent airborne contamination by windswept tailings.

\section{MOISTURE BLOCK DATA}

The moisture blocks were buried a few centimeters below the bottom of each liner. These instruments measure the degree of saturation of the foundation soil, which is equal in percentage to the saturation ratio. These values indicated the effectiveness of each liner as a leachate barrier. Foundation soil moisture contents at various times after installation of the liner materials are shown in Figure 31. 


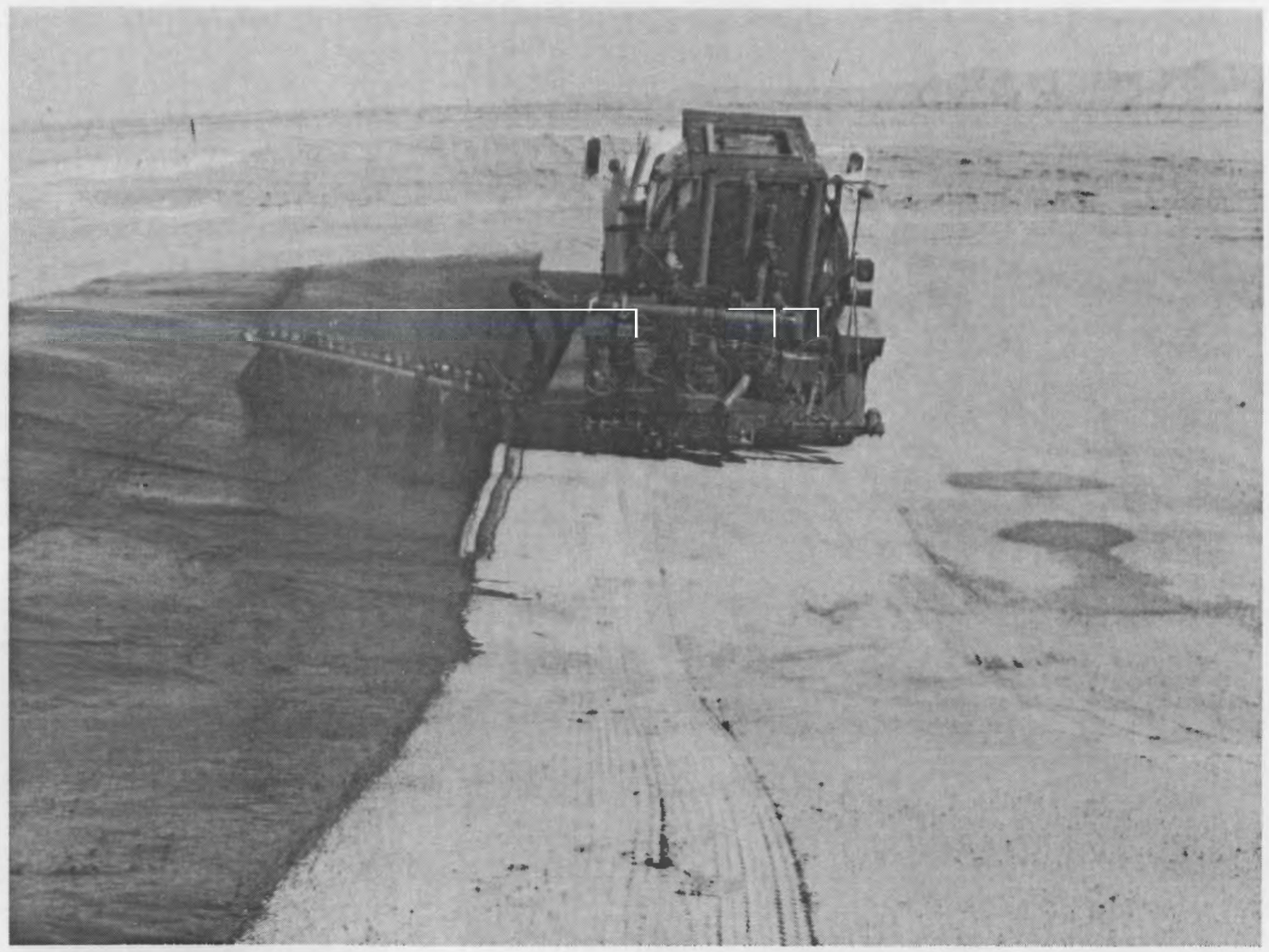

FIGURE 29. Spray Application of Asphalt Membrane 


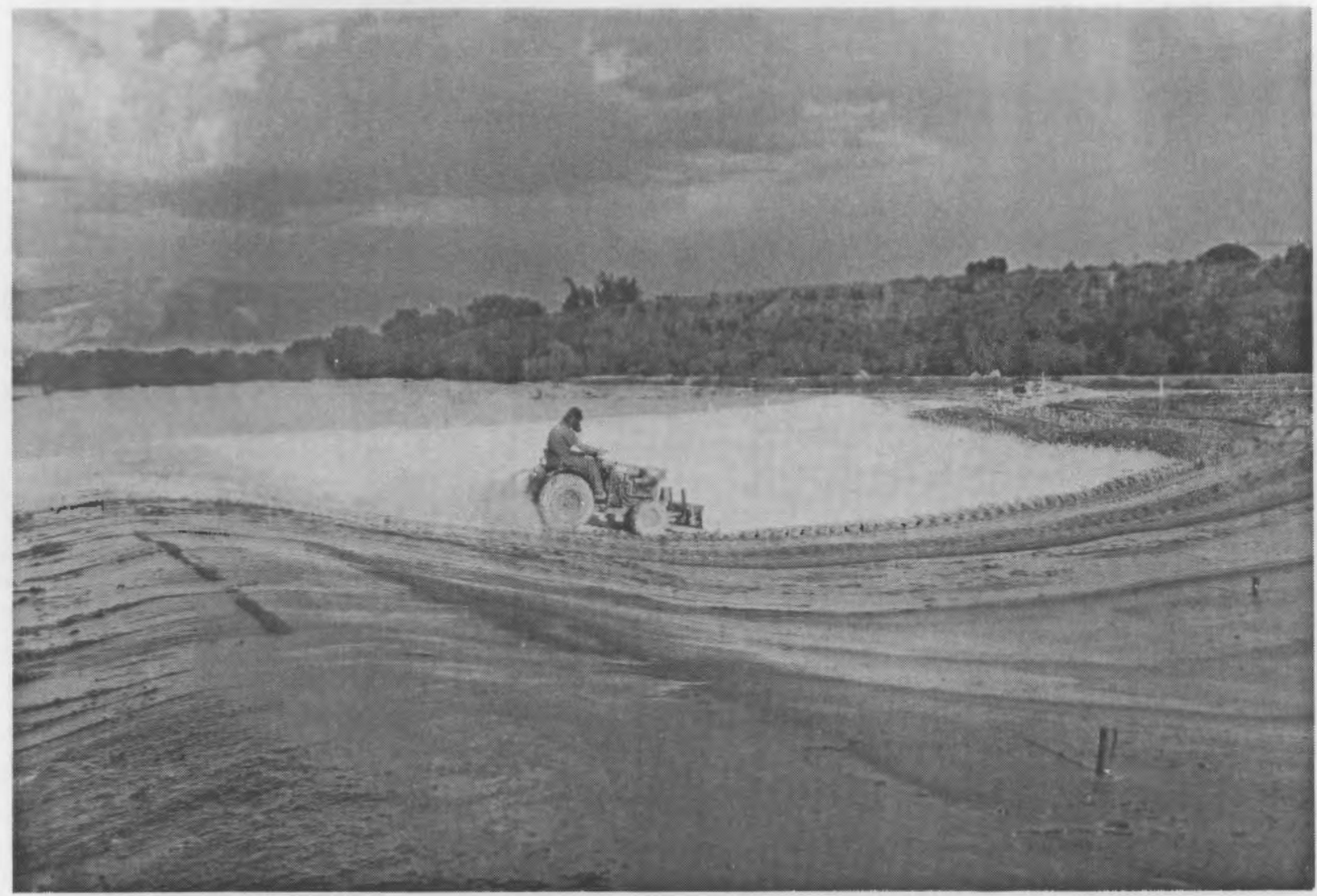

FIGURE 30. Rototilling of Bentonite into Foundation Soil 

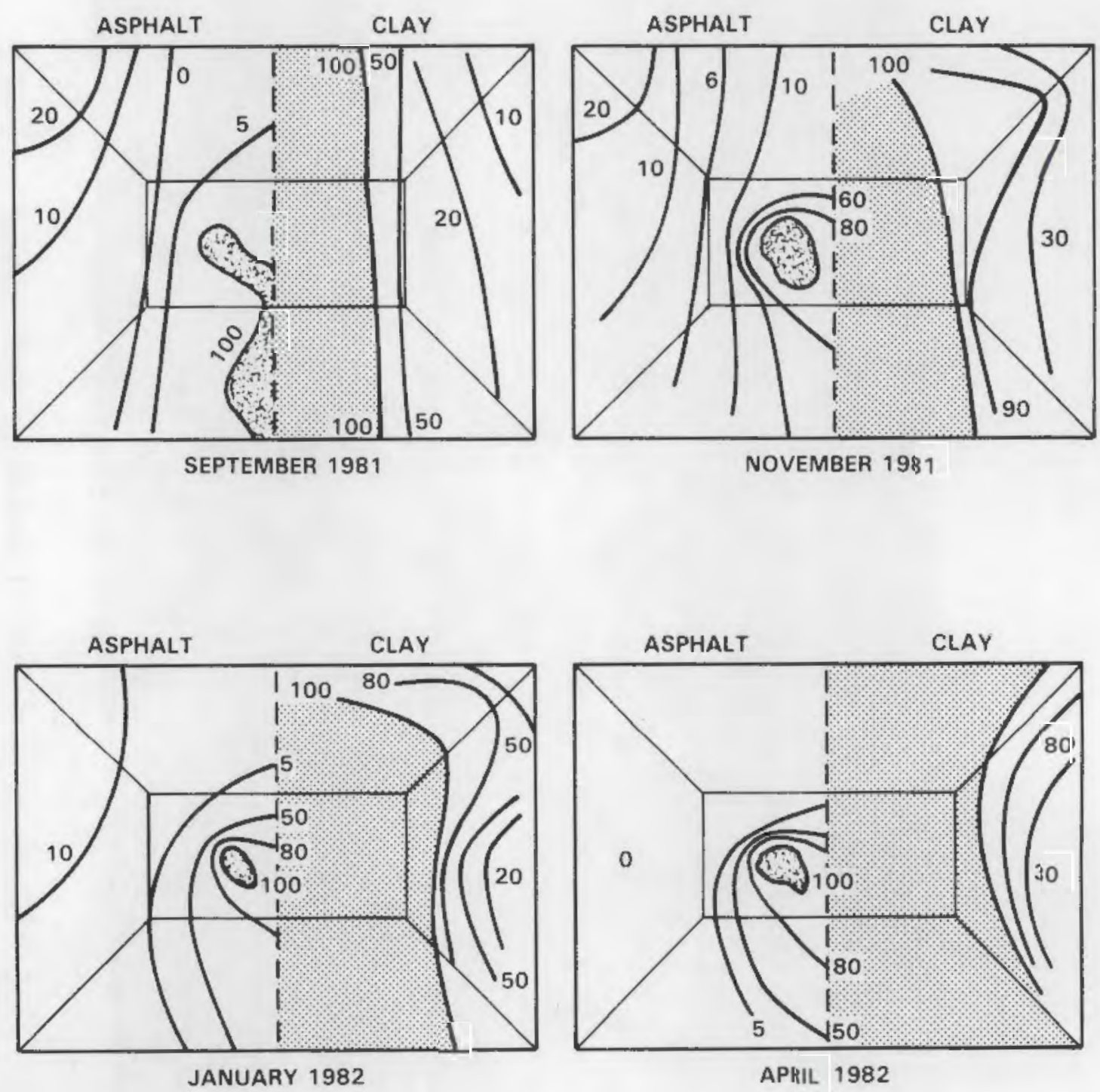

FIGURE 31. Degree of Saturation of Foundation Soil Below the Liners at Selected Times 
The saturated foundation soil condition beneath a portion of the asphalt membrane shortly after installation (September 1981) was caused by misplacement of the polyethylene divider between the foundation soils. Rainfall over the eastern half of the pit during installation of the bentonite liner, and leachate that subsequently migrated through the bentonite liner, moved horizontally through the foundation soil beneath the asphalt membrane. The misplaced polyethylene divider was reinstalled in November 1981 and, as can be seen by the isograms, the foundation soil moisture content began to decrease slowly. Except for a small area near the center, data collected after that time showed that the asphalt membrane had been an effective barrier to leachate migration.

A leak was detected in the center of the pit where an inadequate thickness of asphalt occurred in a one-square-meter area. This was discovered when the liner was partially uncovered and sampled in May 1983. The thin portion of the liner had small-diameter $(1-\mathrm{cm})$ holes that allowed leachate to pass slowly.

On the other hand, the clay liner did not demonstrate the same type of effectiveness as a leachate barrier. It was not expected to be as impermeable, especially for the near-neutral tailings conditions found at Grand Junction. Athough leachates were able to migrate through the clay liner, it was an effective cation exchanger for adsorbing contaminants from the leachates as they passed through. The performance of the clay liner will be discussed in more detail in the following section, which presents data from leachate samples collected above and below the liner.

Figure 32 compares the saturation ratios for selected locations under the asphalt and clay liners. As the figure shows, the degree of saturation under the clay liners rose to almost $100 \%$ in most cases within a few months of saturating the tailings above the liner. Saturation of the foundation soil beneath the asphalt membrane, however, remained relatively constant following the repair of the polyethylene divider. 


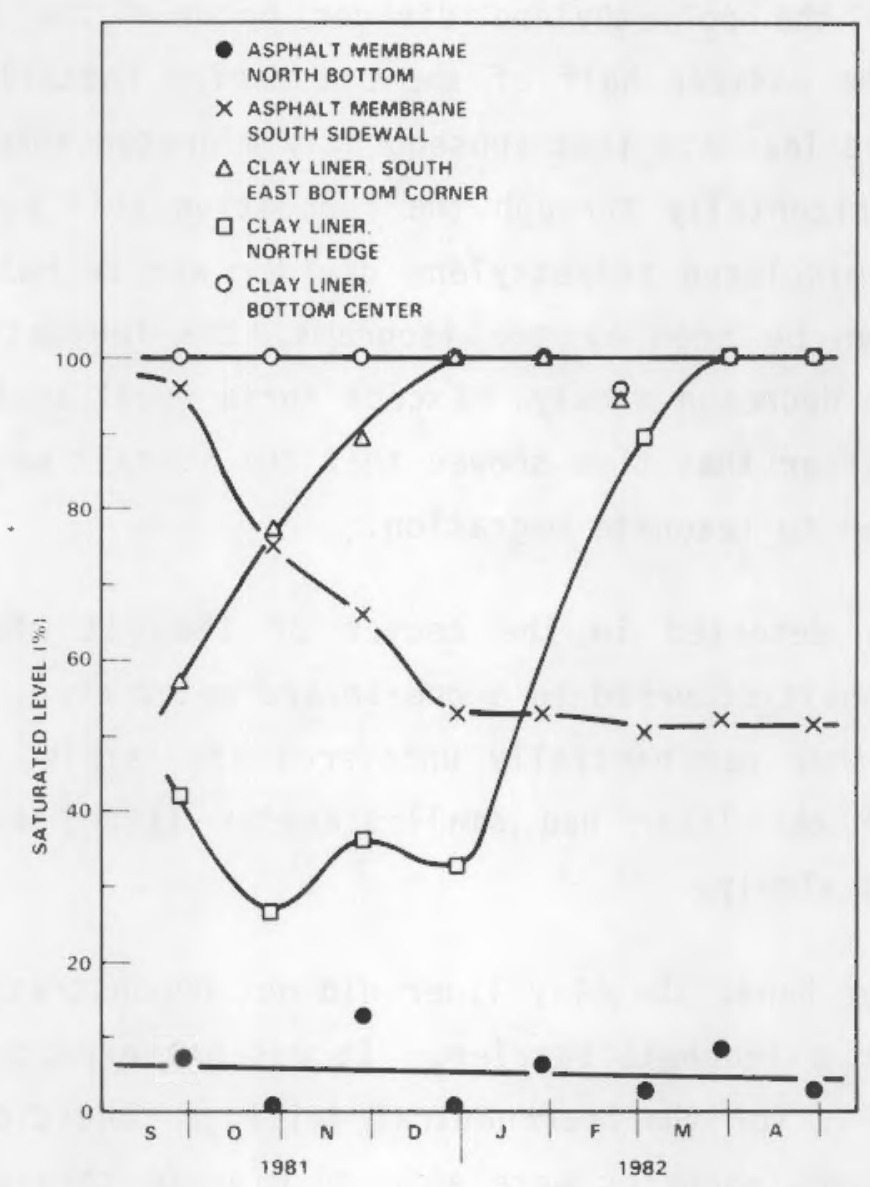

FIGURE 32. Degree of Saturation Under Asphalt and Clay Liners at Selected Locations

Piezometers indicated the water levels in the tailings during this period (see Figure 33). The rapid decrease in water level above the clay liner was caused by evaporation through the cover and leachate permeation through liner. Since the asphalt membrane was much more effective as a leachate barrier, the gradual decrease in water level for the tailings above the membrane must be attributed primarily to evaporation through the cover. In addition, a small portion would have leaked through the $1-\mathrm{cm}$ holes caused by faulty installation in a small area of the liner. 


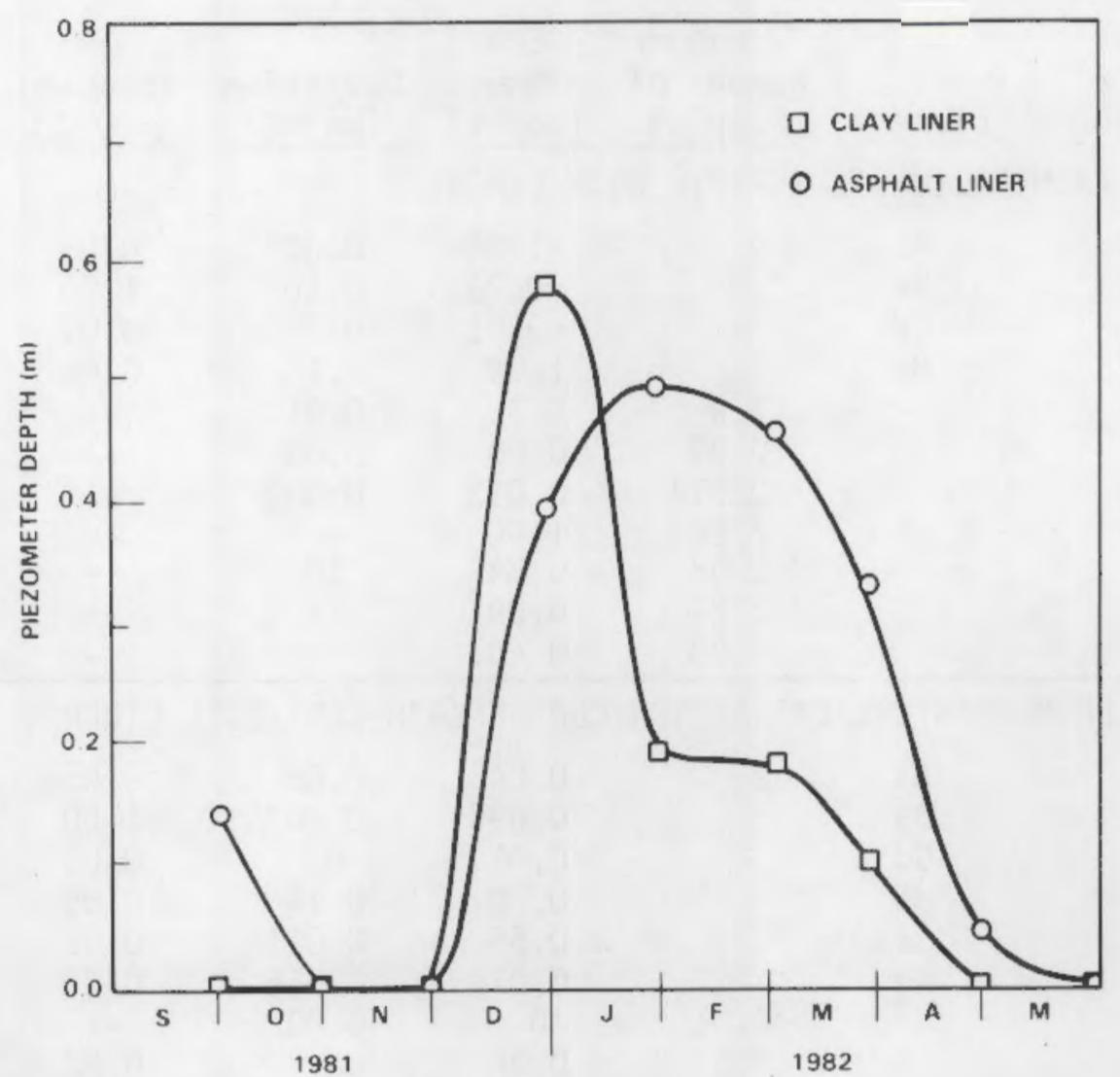

FIGURE 33. Water Level in Tailings Above Asphalt and Clay Liners

\section{LEACHATE SAMPLE ANALYSIS}

Leachate samples were collected from the piezometers in the tailings above the asphalt and clay liners on a monthly basis as well as from the moisture extraction cups beneath the liners when conditions permitted. Analyses of the samples showed that the clay liner was effective as a barrier to contaminants identified in the Environmental Protection Agency (EPA) Drinking Water Standards (U.S. EPA 40 CFR 141).

The results of the piezometer and moisture extraction cup sample analyses are listed in Table 16. The samples were analyzed for elemental As, $\mathrm{Ba}, \mathrm{Cd}, \mathrm{Mo}, \mathrm{Se}, \mathrm{Ag}$, and $\mathrm{Hg}$. Chloride, nitrate, and sulfate anions were also analyzed. The tabular data indicates that the clay liner effectively 
TABLE 16. Leachate Sample Analysis

\begin{tabular}{|c|c|c|c|c|c|c|}
\hline $\begin{array}{c}\text { Sample } \\
\text { Description }\end{array}$ & Element & $\begin{array}{c}\text { Standard } \\
\text { Number of } \\
\text { Samples }\end{array}$ & $\begin{array}{r}\text { EPA } \\
\text { Mean } \\
(\mu \mathrm{g} / \mathrm{mL}) \\
\end{array}$ & $\begin{array}{c}\text { Deviation } \\
(\mu \mathrm{g} / \mathrm{mL})\end{array}$ & $\begin{array}{l}\text { Standard } \\
(\mu \mathrm{g} / \mathrm{mL})\end{array}$ & $\begin{array}{l}\text { Reduction } \\
\text { Factor }\end{array}$ \\
\hline \multicolumn{7}{|c|}{ LEACHATE FROM PIEZOMETERS ABOVE BOTH LINERS } \\
\hline $\begin{array}{l}\mathrm{Se} \\
\mathrm{Ag} \\
\mathrm{Hg}^{-} \\
\mathrm{Cl}^{-} \\
\mathrm{NO}^{-} \\
\mathrm{SO}^{-} 4^{-2} \\
\mathrm{pH}^{-2}\end{array}$ & $\begin{array}{l}\text { As } \\
\mathrm{Ba} \\
\mathrm{Cd} \\
\text { Mo }\end{array}$ & $\begin{array}{c}2.9 \\
0.09 \\
<0.014 \\
2.86 \\
1.08 \\
2.24 \\
7.88\end{array}$ & $\begin{array}{l}1.86 \\
0.23 \\
<0.01 \\
10.9 \\
0.7 \\
0.06 \\
0.013 \\
1.96 \\
0.84 \\
0.29 \\
0.40\end{array}$ & $\begin{array}{l}0.85 \\
0.10 \\
0 \\
2.1 \\
0.01 \\
0.05 \\
0.002 \\
-- \\
10 \\
-- \\
--\end{array}$ & $\begin{array}{l}0.05 \\
1.00 \\
0.01 \\
0.05 \\
-- \\
-- \\
-- \\
-- \\
-- \\
--\end{array}$ & $\begin{array}{l}-- \\
-- \\
-- \\
--\end{array}$ \\
\hline
\end{tabular}

LEACHATE FROM MOISTURE EXTRACTION CUP BENEATH CLAY/SOIL LINER

\begin{tabular}{lcccccc} 
& As & 2 & 0.045 & 0.05 & 0.05 & 41.3 \\
& $\mathrm{Ba}$ & & 0.095 & 0.007 & 1.00 & 2.4 \\
& $\mathrm{Cd}$ & & $<0.01$ & 0 & 0.01 & -- \\
& $\mathrm{Mo}$ & & 0.70 & 0.14 & 0.05 & 15.6 \\
& $\mathrm{Se}$ & & 0.55 & 0.071 & 0.01 & 5.3 \\
& $\mathrm{Ag}$ & & 0.07 & 0.014 & 0.05 & 1.3 \\
$\mathrm{Hg}$ & & $<0.01$ & 0 & 0.002 & -- & \\
$\mathrm{C}-$ & & 3.5 & 0.28 & -- & 0.82 & \\
$\mathrm{NO}_{3}-$ & & 6.4 & 0.78 & 10 & 0.17 & \\
$\mathrm{SO}_{4}-2$ & & 6.4 & 0.42 & -- & 0.36 & \\
$\mathrm{pH}^{4}$ & & 8.11 & 0.10 & -- & -- & \\
\hline
\end{tabular}

LEACHATE FROM MOISTURE CUP BENEATH ASPHALT MEMBRANE

$\begin{array}{lllll}\mathrm{Hg} & <0.01 & 0 & 0.002 & -- \\ \mathrm{Cl}^{-} & 3.2 & 1.1 & -- & 0.89 \\ \mathrm{NO}_{3}^{-} & 1.9 & 0.8 & 10 & 0.57 \\ \mathrm{SO}_{4}^{-2} & 2.7 & 0.3 & -- & 0.83 \\ \mathrm{pH}^{-2} & 8.18 & 0.12 & -- & --\end{array}$


reduced the elemental contaminant concentrations either by factors of 5-50 or to within the drinking water standard.

In the case of $\mathrm{Cd}$, the concentration in the leachate above the liner was below both the analytical detection limit and the standard. Silver reduction was only a factor of 1.3, but the arithmetic mean of $\mathrm{Ag}$ concentrations in the leachate samples below the clay liner was very near the drinking water standard of $0.05 \mu \mathrm{g} / \mathrm{ml}$. In all cases, mercury concentration was below the detection limit of $0.01 \mu \mathrm{g} \mathrm{per} / \mathrm{mL}$. The leachate concentration of $\mathrm{Ba}$ below the clay liner was 2.4 times less than that in the leachate above the liner; however, the concentrations below and above the liner were both below the $\mathrm{MCL}$.

The reduced contaminant concentration is attributed primarily to the sorption of cations onto the sodium bentonite clay minerals. Coprecipitation of contaminants in the leachate was not expected to be a factor in this case because the $\mathrm{pH}$ of the piezometer leachate samples and the moisture extraction cup samples were both near neutral (i.e., between 7.13 and $8.25 \mathrm{pH})$.

Precipitation of cations was not as significant as it would have been for an acidic leachate contacting this soil/clay liner; however, As, Mo, and Se concentrations were reduced. These reductions were not the result of cation exchange because As, Mo, and Se most likely exist as anionic species in these leachates. Anion adsorption is not likely to be important either because of the high $\mathrm{pH}$. The only apparent mechanism left to explain the reduction of $A s, M_{0}$, and Se concentrations is their precipitation in as-yet unidentified compounds. By the process of elimination, therefore, precipitation reactions for some elements do contribute to the effectiveness of soil and clay liners for neutral leachates.

No reduction in contaminant concentrations was observed for chlorides, nitrates, and sulfates. The average chloride concentrations of the piezometer and extraction cup leachates were within each other's standard deviation; thus, the clay liner was not sorbing any of the chloride ions. On 
the other hand, the nitrate and sulfate concentrations below the clay liner were on the order of 3-6 times those of the concentrations in the piezometer leachate--probably due to interactions with the foundation soil as the leachate passed through the liner. The concentrations of sulfates, nitrates, and chlorides in the foundation soil were inconsequential, however, because they were either below the EPA drinking water MCL's or not listed. In the case of higher sulfate concentrations in the leachate, which would occur in primarily acidic tailings, the sulfate concentration would be reduced by precipitation of sulfate compounds as the leachates where neutralized while passing through the clay/soil liner.

Table 16 also lists the leachate concentrations below the asphalt liner. These data show that the concentration of contaminants has not been reduced in the leachate below the asphalt liner to the same degree as the clay liner. The only leachates collected under the asphalt liner were near the leaking section described earlier. The reduction in contaminant concentrations, especially that of arsenic, was probably due to the interaction of the leachate with the foundation soil. Even so, the contaminant reduction was not as pronounced as those leachates that had permeated the clay liner. Thus, the foundation soil alone was apparently not as effective as the combination of bentonite and soil in sorbing and precipitating the clay contaminants from Grand Junction leachates.

\section{ASPHALT LINER EVALUATIONS}

In May 1983, the liners were partially uncovered for sampling. In the west end of the pit, three asphalt liner samples were collected from three separate locations. The samples were analyzed and compared with a laboratory aged sample and an unexposed sample that was taken from the field membrane in September 1981.

A procedure developed by Geir and Morrison (1968) was used to make the following determinations:

- statistical variation of membrane thickness

- thickness of crust formation on the exposed surface

- extent of soil impregnation from the underlying soil. 
One of the locations was selected specifically to determine the cause for the high saturation ratios detected under a portion of the liner. It was discovered that, due to improper application and inspection practices, a one-square-meter portion of the liner had been applied with insufficient asphalt. This resulted in holes approximately $1 \mathrm{~cm}$ in diameter.

According to the Geir and Morrison procedure, this sample received a fair to poor rating, primarily because of its discontinuity. The other two samples, which had very few surface cracks, a thin layer of crust, good flexibility, and no physical damage, earned good ratings.

Table 17 compares the three field samples and the laboratory-aged and virgin samples. The table shows that the two properly installed field samples compared very well with the laboratory-aged samples, supporting the conclusion that the aging tests were successful in accelerating the degradation reactions in the laboratory.

TABLE 17. Comparison of Field, Virgin, and Laboratory-Aged Asphalt Liners

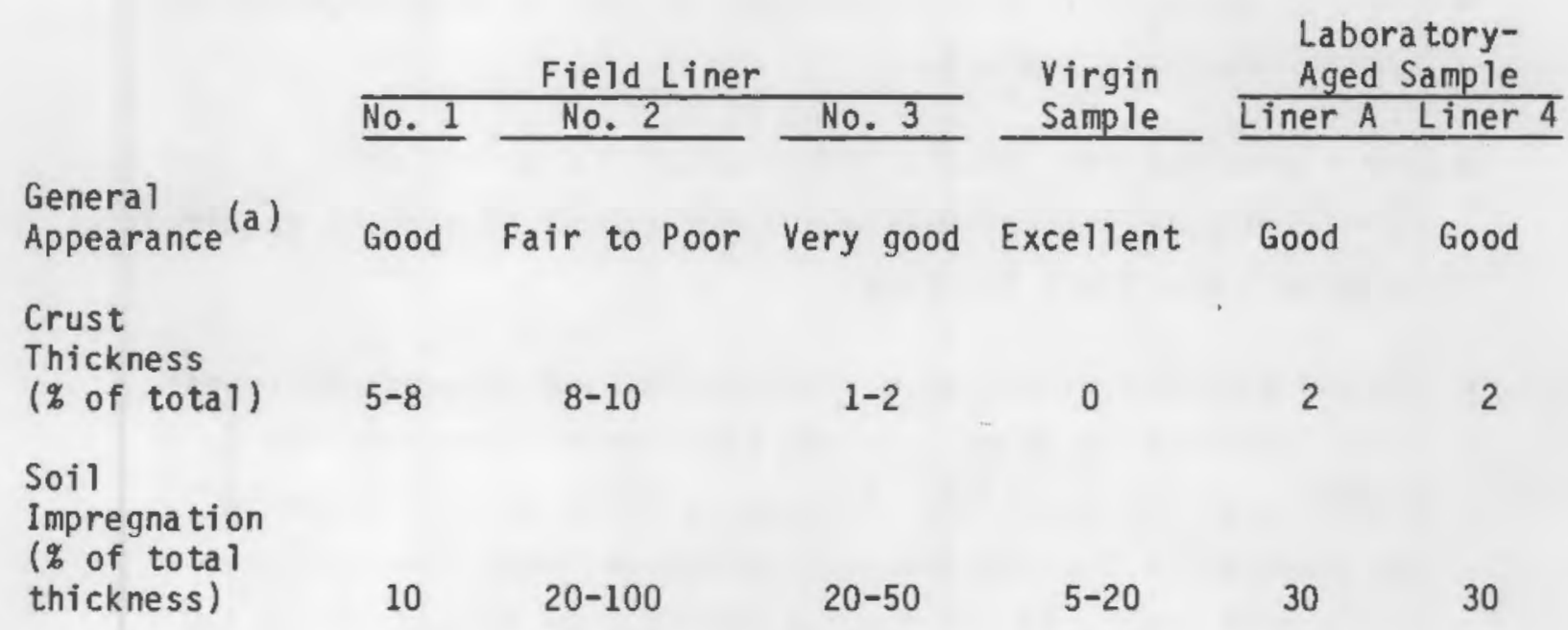

(a) Good is defined as few surface cracks, thin layer of crust, flexible, and small amount of physical damage. Fair is defined as some surface cracking with a few large cracks, a portion of the surface crust brittle, some foreign material incorporated, and some physical damage. Poor is defined as brittle and lifeless, large amount of foreign material incorporated, and extensive phusical damage (Geir and Morrison 1968). 
The uniformity of thickness of the three field samples was also measured at 10 random locations, each to determine the adequacy of application. The average thicknesses for liner samples 1,2 , and 3 were $6.33 \mathrm{~mm}$ ( standard deviation $=2.43), 4.24 \mathrm{~mm}$ (standard deviation $=1.46 \mathrm{~mm}$ ), and $7.17 \mathrm{~mm}$ (standard deviation $=1.94 \mathrm{~mm}$ ), respectively. Only liner 2, which was inappropriately applied, was significantly below the design thickness of $7 \mathrm{~mm}$.

The experience with the formation of an inferior portion of the liner during installation demonstrated the need for appropriate design and inspection practices. Recommended construction procedures for buried asphalt membranes are outlined by Geir and Morrison (1968) and Van Asbeck (1964) and are summarized below.

- The maximum preferred steepness of a side slope is two horizontal to one vertical. The limiting factor is stability of the foundation soil and preparation and application equipment. The demonstrated slope thickness during the field tests was $4: 1$, which should be attained if at all practicable.

- The foundation soil must be dragged to remove sharp objects and expose rocks for removal.

- The foundation soil should be rolled with a smooth steel roller to minimize soil impregnation and the amount of asphalt required to obtain a uniform thickness.

- If the subgrade is dusty, a light sprinkling of water is necessary. Failure to do this in the 1981 field tests resulted in a 10-50\% soil impregnation. Although a thick coat of cationic emulsion prior to membrane application has been used in other field tests, this is not recommended because it does not allow access by the asphait distributor truck.

- The asphalt must meet the specifications outlined in Table B.3 (Appendix B) and use a $\mathrm{P}_{2} \mathrm{O}_{5}$ catalyst during the manufacturing process. 
- The asphalt must be applied at $204^{\circ} \mathrm{C}$ with a slot-type spray bar in a minimum of two and preferably three passes. Multiple passes reduce soil impregnation and reseal pinholes from escaping moisture.

- Recommended application speed is $6.5 \mathrm{~km} / \mathrm{h}$.

- Visual inspection of the entire surface is necessary.

- If tailings are not added to the pit immediately after application, a $30-\mathrm{cm}$ thick protective soil cover is recommended to protect the membrane from sunlight, high temperatures, heavy equipment, and vectors.

\section{CLAY LINER EVALUATION}

Six core samples were removed from the clay liner field test pit in May 1983. The cores were analyzed to determine the effects of field conditions on the chemical and physical properties of the test liner. All cores were collected in clear lexand tubing so that core quality could be monitored in the field prior to shipment back to the laboratory for analysis.

Three cores contained only the clay liner portions of the overall liner system and measured $8.10 \mathrm{~cm}$ in length and $8.86 \mathrm{~cm}$ in diameter. Each of the remaining three cores, which measured $30.5 \mathrm{~cm}$ in length and $8.86 \mathrm{~cm}$ in diameter, contained material from the three layers of the liner test system: leached tailings, clay liner, and foundation soil.

Bulk tailings samples contained leached tailings from directly above the clay liner and undisturbed tailings collected adjacent to the test pit. In addition, a sample of laboratory-prepared clay liner was produced from nine parts uncontacted foundation soil and one part Wyoming-grade sodium bentonite. A brief description of each core is provided in Table 18. 
TABLE 18. Field Sample Description

Sample Number

Core No. 1

Core No. 2

Core No. 3

Core No. 4

Core No. 5

Core No. 6

Leached Tailings

Fresh Tailings
Core Length and Description

8.1-cm clay liner core

8.1-cm clay liner core

8.1-cm clay liner core

30.48-cm core containing leached tailings, clay liner, foundation soil

30.48- $\mathrm{cm}$ core containing leached tailings, clay liner, foundation soil

30.48-cm core containing leached tailings, clay liner, foundation soil

Leached tailings collected from above the clay liner from sampling hole No. 4

Uncontacted tailings collected adjacent to the liner site

Each column was subsampled into five equal lengths:

Column top $-24.4 \mathrm{~cm}$

$24.4 \mathrm{~cm}-18.3 \mathrm{~cm}$

$18.3 \mathrm{~cm}-12.2 \mathrm{~cm}$

$12.2 \mathrm{~cm}-6.1 \mathrm{~cm}$

$6.1 \mathrm{~cm}$ - column bottom.

The average depth of the clay liner from the column top was $22 \mathrm{~cm}$. The first two subsamples (column top $-24.4 \mathrm{~cm}$ and $24.4 \mathrm{~cm}-18.3 \mathrm{~cm}$ ) contain mostly leached tailings. Assuming a clay liner thickness of $10 \mathrm{~cm}$, the third subsample $(18.3 \mathrm{~cm}-12.2 \mathrm{~cm})$ was predominantly clay liner. The last two subsamples contained foundation soil.

Sediment Analysis

The optimum physical properties of the clay liner were tested using laboratory-produced liner material, which was tested for optimal proctor density and particle size distribution by standard ASTM methods. For comparison, contacted clay liner material obtained from Core No. 3 was also tested for field water content, dry bulk density, and particle size distribution. Results of these tests indicated the effectiveness of preparing 
and installing a clay liner under field conditions. Measurements of hydraulic conductivity were made using Cores No. 1 and No. 2, by a modified ASTM falling head permeability method (ASTM 1978). Flow through the cores was from bottom to top to ensure saturated conditions.

Solution Analysis

Solutions were prepared from every sediment sample by the method of 1:1 extraction with distilled water. Extracts were produced by adding a known weight of sediment to an equal weight of distilled water. The resulting slurry was placed in $0.5-\mathrm{L}$ polyethylene bottles and shaken for 48 hours on a linear oscillating shaker to facilitate mixing. Upon completion of the two-day contact period, samples were centrifuged and supernates were filtered through a 0.45-micron membrane. Each $1: 1$ extract sample was split into four aliquots for analysis. Ten milliliters were sent for $\mathrm{pH}$ and $E \mathrm{~h}$ determination, 10 to cation analysis, 10 to anion analysis, and at least 200 to radionuclide analysis.

Radionuclide concentrations were determined by high-resolution gamma spectroscopy. For this analysis, a known volume of solution (at least 200 $\mathrm{mL})$ was evaporated. The resulting evaporates were mixed with a cellulose Dinder and pressed into a pellet of fixed geometry. A Princeton Gamma-Tech intrinsic germanium diode coupled with a Nuclear Data ND660 nuclear analyzer was used for all counting. 



\section{RESULTS AND DISCUSSION}

PHYSICAL CHARACTERISTICS

The results of proctor testing and particle size analysis for the laboratory-prepared clay liner are presented in Figures 34 and 35, respectively. Particle size distribution of the contacted field liner obtained from Core No. 3 is given in Figure 36. For comparison, the water content, dry bulk density, and particle size distribution data for both samples are presented in Table 19.

The results listed in Table 19 indicate that field preparation and installation of the test clay liner were very successful. Although the field liner carries a soil classification of loam and the laboratoryprepared liner a classification of silt loam, particle size distribution data showed the two mixes to be very similar. Under the unified soil classification system, both liner materials were classified as fine-grained inorganic silts $(M L)$. This indicated that a properly proportioned mixture was achieved under field conditions.

In addition, the dry bulk density of the field test liner was within $88 \%$ of proctor, as determined by testing of the laboratory-prepared liner. Along with a field water content of 20.4 weight percent, the proctor data indicated that the liner could be compacted to, and maintain, even higher bulk density values in the field. An increase in field bulk density would result in decreased liner permeability and increased liner resistance to leachate migration.

Hydraulic conductivity data obtained from Cores No. 1 and 2 showed permeability ranging from $1 \times 10^{-7} \mathrm{~cm} / \mathrm{s}$ to $5 \times 10^{-9} \mathrm{~cm} / \mathrm{s}$, respectively. Dry bulk density calculations for each core placed Core No. 1 at $93 \%$ of proctor (bulk density $=1.64 \mathrm{~g} / \mathrm{cm}^{3}$ ) and Core No. 2 at $100 \%$ of proctor (bulk density $=1.87 \mathrm{~g} / \mathrm{cm}^{3}$ ). Bulk density and water content analyses of the subsamples removed from Cores 4,5 , and 6 showed bulk densities ranging from $85-95 \%$ proctor and water contents between 20 and 22 weight percent. 


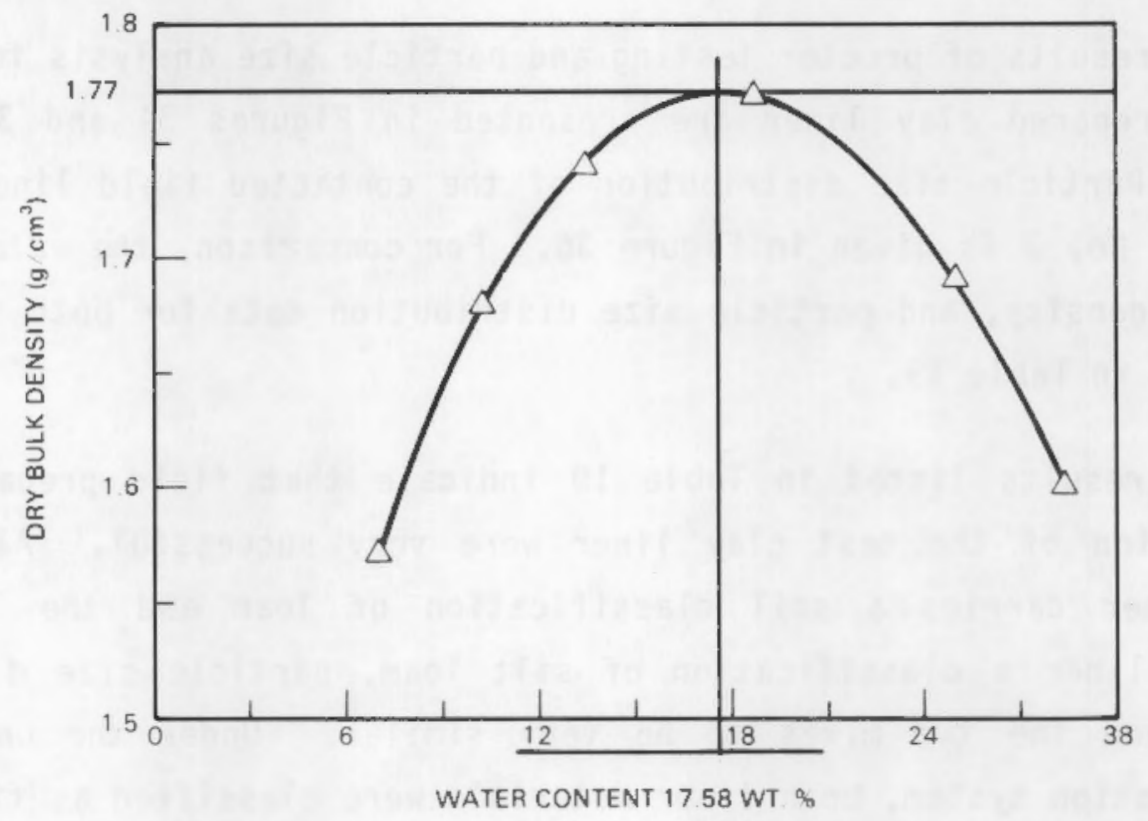

FIGURE 34. Moisture Density Relationship of Laboratory Prepared Clay Liner

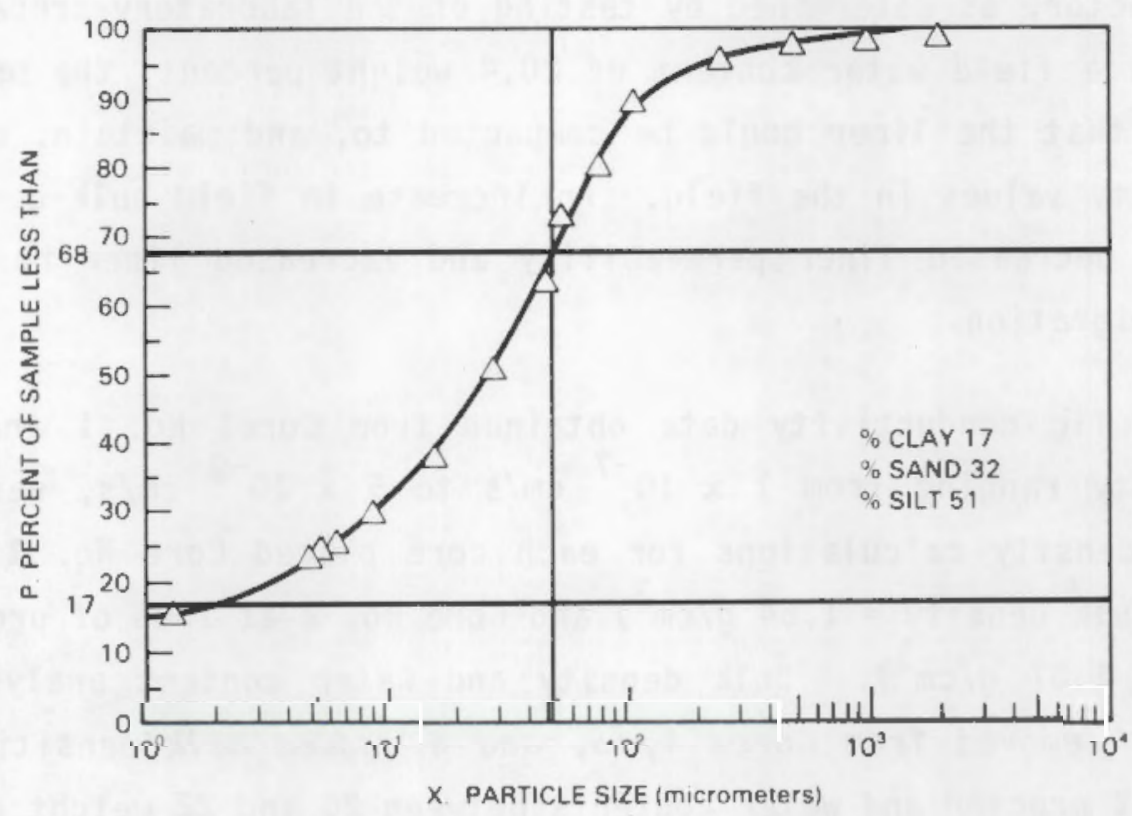

FIGURE 35. Particle Size Distribution of Laboratory Prepared Clay Liner 


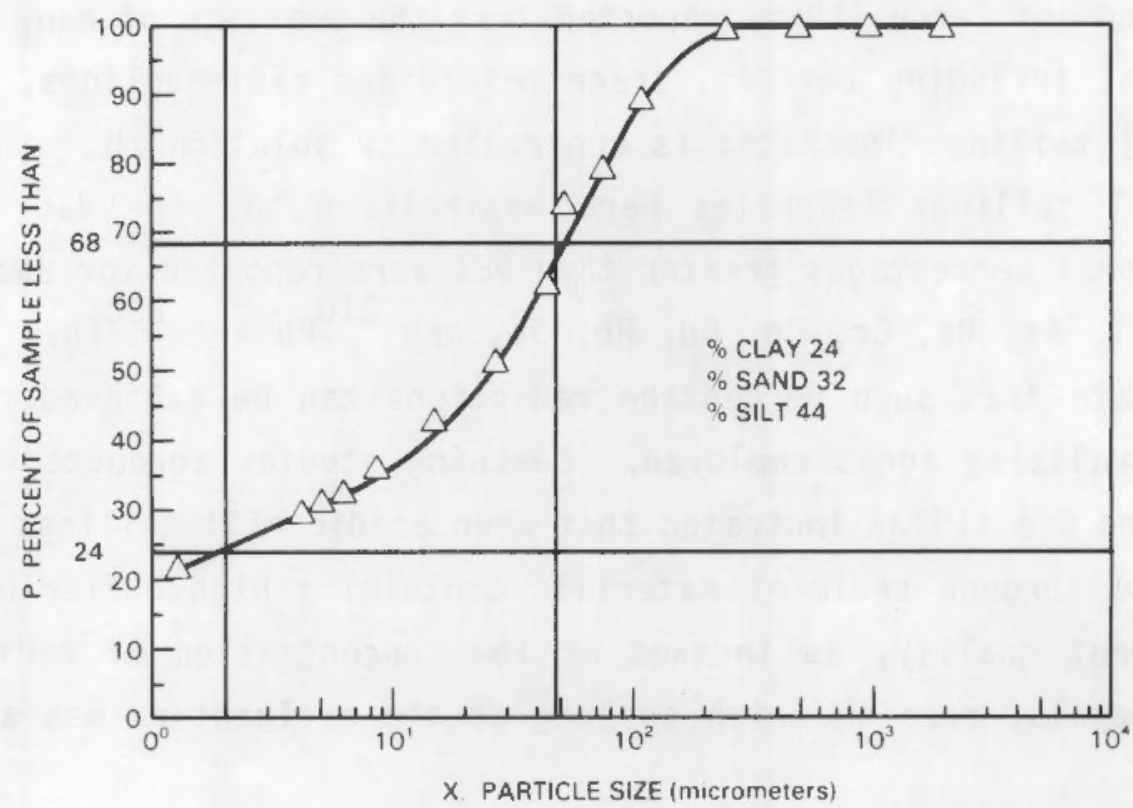

FIGURE 36. Particle Size Distribution of Field Test Clay Liner

TABLE 19. Physical Characteristics of Field and Laboratory Clay Liners

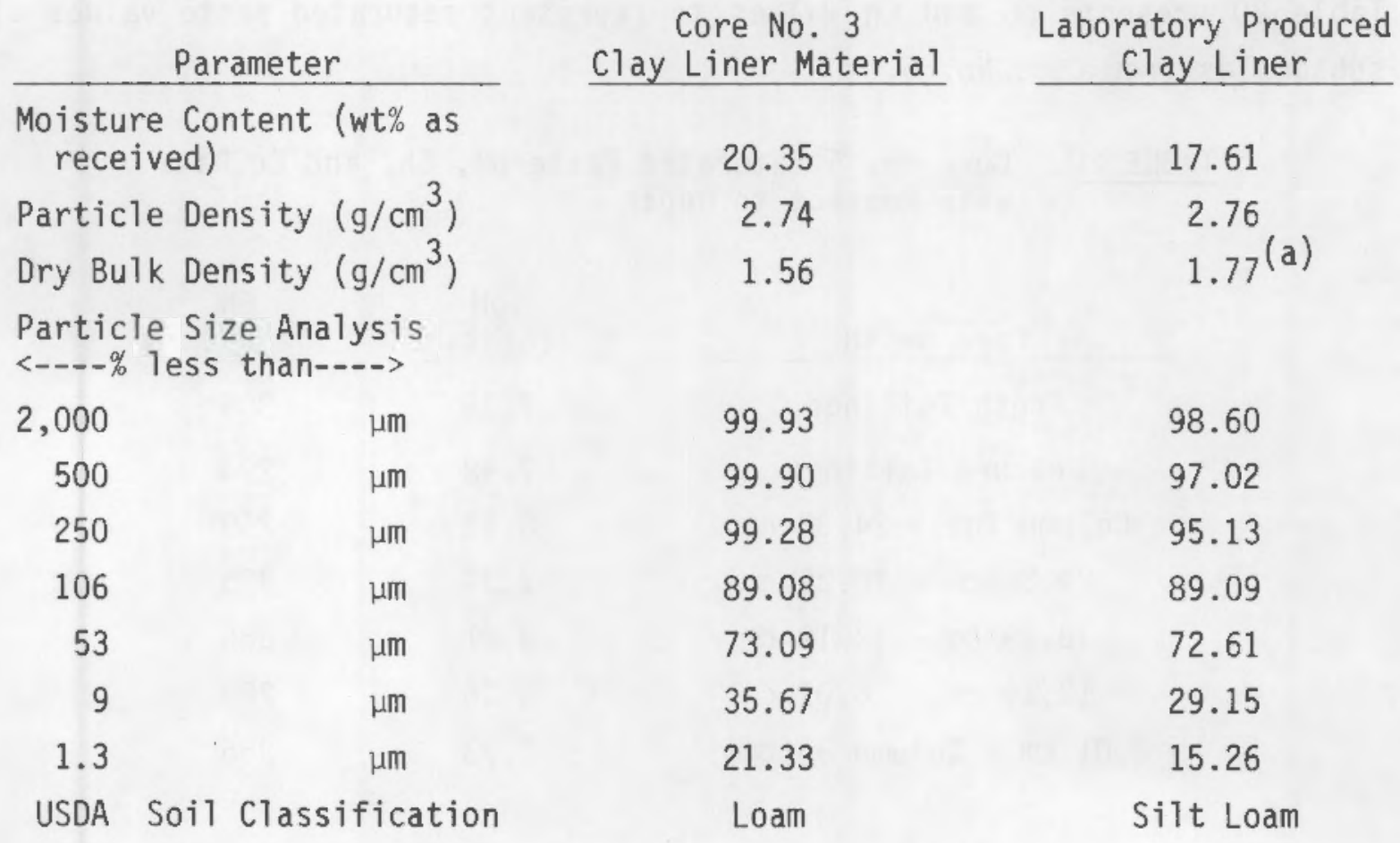

(a) Values represent soil at $100 \%$ Proctor. 


\section{Clay Liner Attenuation Characteristics}

Sherwood and Serne (1983) reported that the mobility of many dissolved constituents, including cations, trace metals and radionuclides, in acidic uranium mill tailings leachates is controlled by solution pH. When acidic uranium mill tailings leachates were neutralized to $\mathrm{pH}=7.0$ or above, average removal percentages greater than $98 \%$ were reported for such contaminants as $\mathrm{Al}, \mathrm{As}, \mathrm{Ba}, \mathrm{Cr}, \mathrm{Co}, \mathrm{Cu}, \mathrm{Pb}, \mathrm{Si}$, and ${ }^{210} \mathrm{~Pb}$ and ${ }^{230} \mathrm{Th}$. These results indicate that such percentage reductions can be achieved regardless of the neutralizing agent employed. Leaching studies conducted by Serne, Peterson, and Gee (1983) indicated that when acidic mill tailings effluents were leached through sediment materials containing high buffering capacities, effluent quality, as indexed by low concentration of radionuclides and heavy metals, remained high as long as the effluent $\mathrm{pH}$ was above $\mathrm{pH}=$ 4.0 .

As a first check of the potential level of contaminant migration through the Grand Junction test clay liner, $\mathrm{pH}$ was monitored with dep.th through the liner system. The results of this investigation are found in Table 20, which also includes the pH of uncontacted and leached tailings. Table 20 presents $\mathrm{pH}$ and $\mathrm{Eh}$ values to represent saturated paste values of subsamples from Core No. 5 .

TABLE 20. Core No. 5 Saturated Paste pH, Eh, and Ec Data with Respect to Depth

\begin{tabular}{|c|c|c|}
\hline Core Depth & $\begin{array}{c}\mathrm{pH} \\
\text { (units) } \\
\end{array}$ & $\begin{array}{c}E h \\
(m V)\end{array}$ \\
\hline Fresh Tailings & 7.39 & 305 \\
\hline Leached Tailings & 7.98 & 294 \\
\hline Column Top $-24.38 \mathrm{~cm}$ & 8.51 & 259 \\
\hline $24.38 \mathrm{~cm}-18.29 \mathrm{~cm}$ & 7.34 & 335 \\
\hline $18.29 \mathrm{~cm}-12.19 \mathrm{~cm}$ & 8.01 & 265 \\
\hline $12.19 \mathrm{~cm}-6.01 \mathrm{~cm}$ & 8.36 & 283 \\
\hline $6.01 \mathrm{~cm}$ - Column Bottom & 7.73 & 256 \\
\hline
\end{tabular}


TABLE 21. 1:1 Extract pH, Eh, and Ec Data with Respect to Depth

\begin{tabular}{cccc} 
Core Depth & & $\begin{array}{c}\text { pH } \\
\text { (units) }\end{array}$ & $\begin{array}{c}\text { Eh } \\
\text { (mV) }\end{array}$ \\
\cline { 1 - 1 } Fresh Tailings & & 7.87 & 301 \\
Leached Tailings & & 8.28 & 291 \\
Column Top $-24: 38 \mathrm{~cm}$ & & 8.05 & 260 \\
$24.38 \mathrm{~cm}-18.29 \mathrm{~cm}$ & & 7.92 & 282 \\
$18.29 \mathrm{~cm}-12.19 \mathrm{~cm}$ & & 7.89 & 268 \\
$12.19 \mathrm{~cm}-6.01 \mathrm{~cm}$ & & 8.18 & 278 \\
$6.01 \mathrm{~cm}-$ Column Bottom & 7.60 & 277
\end{tabular}

Results of $\mathrm{pH}, \mathrm{Eh}$, and $\mathrm{Ec}$ data taken for $1: 1$ extract solutions are given in Table 21. The $\mathrm{pH}$ and $\mathrm{Eh}$ values are average $1: 1$ extraction values obtained from the analysis of 1:1 extracts of subsamples of Cores 4,5 , and 6.

From the $\mathrm{pH}$ data presented for the test liner system, it is evident that neutral pH conditions exist throughout the liner system. Thus, low leachate concentrations can be expected of all contaminant species displaying solubility restrictions at neutral pH. Because several of the contaminating constituents of the leachate source and the clay liner material will be insoluble at the test liner system's neutral pH, X-ray fluorescence, rather than 1:1 extract analysis, provided a greater understanding of the overall contamination source presented by the solid tailings. X-ray fluorescence data for uncontacted tailings, leached tailings, and laboratory prepared clay liner are shown in Table 22.

Results of X-ray fluorescence analysis showed $\mathrm{As}, \mathrm{Cl}, \mathrm{Cr}, \mathrm{Cu}, \mathrm{Mo}, \mathrm{Ni}$, $\mathrm{Pb}, \mathrm{U}, \mathrm{V}$, and $\mathrm{Zn}$ were present in the uncontacted tailings and leached tailing at concentrations several orders of magnitude higher than the uncontacted liner. With the exception of As, these constituents indicated reduced concentrations in the leached tailings in comparison with the uncontacted tailings, suggesting they may have been removed from the contacted tailings as part of the leachate front. 
TABLE 22. X-Ray Fluoresence Data Indicating Concentrations of Solid Material for Uncontacted and Leached Tailings and Laboratory Clay Liner

\begin{tabular}{cccc} 
Parameter & $\begin{array}{c}\text { Uncontacted } \\
\text { Tailings, ppm }\end{array}$ & $\begin{array}{c}\text { Leached } \\
\text { Tailings, ppm }\end{array}$ & $\begin{array}{c}\text { Laboratory } \\
\text { Clay Liner, ppm }\end{array}$ \\
\cline { 2 - 4 } Al & 49,800 & 47,300 & 40,090 \\
As & 113.9 & 373 & 7.21 \\
$\mathrm{Br}$ & $<0.91$ & $<1.1$ & 2.12 \\
$\mathrm{Ca}$ & 36,900 & 27,600 & 90,900 \\
$\mathrm{Cr}$ & 2,640 & 640 & 610 \\
$\mathrm{Co}$ & 27 & 24 & 25 \\
$\mathrm{Cr}$ & 105 & 66 & 34 \\
$\mathrm{CU}$ & 56 & 21.9 & 13.9 \\
$\mathrm{Fe}$ & 20,900 & 18,380 & 16,300 \\
$\mathrm{~K}$ & 8,620 & 13,250 & 11,030 \\
$\mathrm{Mn}$ & 49.9 & 115 & 184 \\
$\mathrm{Mo}$ & 10.4 & 8.5 & 4.4 \\
$\mathrm{Ni}$ & 253 & 133 & 16.6 \\
$\mathrm{P}$ & $<0.33$ & $<0.33$ & $<0.33$ \\
$\mathrm{~Pb}$ & 201 & 76 & 14 \\
$\mathrm{~S}$ & 26,100 & 13,220 & 44,500 \\
$\mathrm{Si}$ & 285,000 & 300,000 & 217,000 \\
$\mathrm{Sr}$ & 323 & 168 & 397 \\
$\mathrm{Ti}$ & 2,300 & 2,100 & 1,780 \\
$\mathrm{U}$ & 201 & 57.5 & 4.9 \\
$\mathrm{~V}$ & 1,683 & 1,101 & 36 \\
$\mathrm{Zn}$ & 109.5 & 87.2 & 174 \\
$\mathrm{Zr}$ & 201 & &
\end{tabular}


On the other hand, concentrations of $\mathrm{Ca}, \mathrm{K}, \mathrm{Fe}, \mathrm{Mn}$, and $\mathrm{S}$ (most likely in the form of sulfates) are conspicuously high in the uncontacted clay liner. The elevated sulfate and iron concentrations in the uncontacted clay liner could mask any movement of these constituents through the liner system. The two-fold drop in sulfate concentration in the leached tailings extracts in comparison with the uncontacted tailings suggests sulfate movement out of the tailings. X-ray fluorescence data indicating concentrations of solid materials for Core No. 5 subsample are shown in Table 23.

The X-ray fluorescence data for Core No. 5 subsamples showed elevated concentrations of $\mathrm{As}, \mathrm{Cl}, \mathrm{Cr}, \mathrm{Cu}, \mathrm{Ni}, \mathrm{Pb}, \mathrm{U}$, and $\mathrm{V}$ above the liner. Of these species only $\mathrm{Cl}$ and $\mathrm{Pb}$ maintained elevated concentrations below the clay liner. In particular, As, $N i, U$, and $V$ either remained insoluble or were well attenuated by the clay liner. Again, levels of $\mathrm{Ca}, \mathrm{K}$, and $\mathrm{Mn}$ were higher in the clay liner and foundation soil than in the solid tailings.

Chemical analyses of the 1:1 extracts produced for uncontacted tailings, leached tailings, laboratory-produced clay liner and uncontacted overburden are given in Table 24, which includes the results of radionuclide counting on solid samples and evaporite salts of each tailings sample. Analytical results were similar to those obtained by X-ray fluorescence. Constituents $\mathrm{As}, \mathrm{Cl}, \mathrm{K}, \mathrm{Mg}, \mathrm{Mo}, \mathrm{Sr}, \mathrm{Si}, \mathrm{V}$, and $\mathrm{Zn}$ were present in solution at elevated concentrations from the tailings extracts in comparison with the uncontacted liner and foundation soil extracts. In addition, $\mathrm{Cl}, \mathrm{K}, \mathrm{Mg}, \mathrm{Mo}, \mathrm{Na}, \mathrm{Ni}, \mathrm{SO}_{4}, \mathrm{Sr}, \mathrm{V}$, and $\mathrm{Zn}$ appeared in higher concentration in the uncontacted tailings extract than the extract produced from leached tailings, suggesting that these species may be mobile in the system.

With the exception of ${ }^{226} \mathrm{Ra}$, radionuclide counting showed that a $1-$ though measurable levels and radionuclide contamination were present in the solid samples, their solubility was very limited at the neutral pH of the liner system. The ${ }^{226} \mathrm{Ra}$ present in the extract of the uncontacted tailings extract seemed rather anomalous, considering that the levels in the leached tailings extract were below detection limits and both samples had equal concentrations in the solid samples. 
TABLE 23. X-Ray Fluorescence Data for Core No. 5 Subsamples

\begin{tabular}{|c|c|c|c|c|c|}
\hline $\begin{array}{c}\text { Parameter } \\
\text { (ppm) }\end{array}$ & $\begin{array}{c}\text { Column Top } \\
\text { to } \\
24.38 \mathrm{~cm} \\
\end{array}$ & $\begin{array}{r}24.38 \mathrm{~cm} \\
\text { to } \\
18.29 \mathrm{~cm} \\
\end{array}$ & $\begin{array}{r}18.29 \mathrm{~cm} \\
\text { to } \\
12.19 \mathrm{~cm} \\
\end{array}$ & $\begin{array}{c}12.19 \mathrm{~cm} \\
\text { to } \\
6.10 \mathrm{~cm} \\
\end{array}$ & $\begin{array}{r}6.10 \mathrm{~cm} \\
\text { to } \\
\text { Column Bottom } \\
\end{array}$ \\
\hline A1 & 36100 & 50700 & 50000 & 50300 & 56000 \\
\hline As & 76.1 & 62.6 & 13.5 & 8.5 & 8.9 \\
\hline$B r$ & $<1.0$ & $<1.0$ & 1.24 & 1.22 & $<1.0$ \\
\hline $\mathrm{Ca}$ & 25,500 & 25,900 & 33,200 & 31,900 & 56,700 \\
\hline $\mathrm{Cl}$ & 620 & 680 & 720 & 640 & 620 \\
\hline Co & $<22$ & $<24$ & $<26$ & $<27$ & $<28$ \\
\hline $\mathrm{Cr}$ & 51 & 60 & 54 & 36 & 43 \\
\hline $\mathrm{Cu}$ & 22.1 & 19.8 & 20.6 & 19.7 & 18.0 \\
\hline $\mathrm{Fe}$ & 15,140 & 17,560 & 20,100 & 22,200 & 22,400 \\
\hline k & 12,510 & 17,220 & 16,960 & 16,090 & 15,180 \\
\hline Mn & 109 & 155 & 188 & 210 & 223 \\
\hline Mo & 9.1 & 6.3 & 7.4 & 5.9 & 5.5 \\
\hline $\mathrm{Ni}$ & 58.5 & 72.2 & 21.6 & 21.4 & 18.2 \\
\hline$P$ & 3000 & 3100 & 3100 & 3100 & 3300 \\
\hline $\mathrm{Pb}$ & 62.4 & 58.1 & 39.2 & 30.4 & 25.9 \\
\hline S & 9740 & 9070 & 7580 & 8620 & 7350 \\
\hline $\mathrm{Si}$ & 322,000 & 308,000 & 287,000 & 281,000 & 270,000 \\
\hline $\mathrm{Sr}$ & 144 & 156 & 146 & 155 & 199 \\
\hline $\mathrm{Ti}$ & 1770 & 1990 & 2290 & 2220 & 2270 \\
\hline$U$ & 44.1 & 32.5 & 6.7 & 5.3 & 5.0 \\
\hline$y$ & 785 & 565 & 79 & 48 & 47 \\
\hline $\mathrm{Zn}$ & 97.6 & 95.4 & 82.3 & 83.7 & 79.1 \\
\hline $2 r$ & 202 & 210 & 218 & 223 & 220 \\
\hline
\end{tabular}


TABLE 24. Chemical Analysis of Tailings and Uncontacted Liner Material: $1: 1$ Extracts

Macro ions (mg/L)

A1

B

Ca

$\mathrm{Fe}$

K

$\mathrm{Mg}$

$\mathrm{Na}$

Si

$\mathrm{Sr}$

$\mathrm{Cl}$

$\mathrm{NO}_{3}$

$\mathrm{SO}_{4}$

Trace metals $(\mathrm{mg} / \mathrm{L})$

As

Ba

$\mathrm{Cr}$

Mo

$\mathrm{Ni}$

$\mathrm{Se}$

$v$

$\mathrm{Zn}$

Radionuclides solid samples ( $\mathrm{pCi} / \mathrm{g}$ )

${ }^{230} \mathrm{Th}$

$210 \mathrm{pb}$

$226 \mathrm{Ra}$

$235 \mathrm{U}$

Radionuclides 1:1 Extracts ( $\mathrm{pCi} / \mathrm{L}$ )

230 Th

$210 \mathrm{pb}$

${ }^{226} \mathrm{Ra}$

$235 u$

(a) $\mathrm{ND}=$ Not Determined

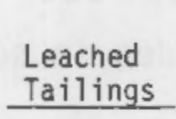

0.05

0.43

804

0.07

37.1

89.4

259.3

33.0

4.91

170

No(a)

2,500

1.56

0.04

0.06

1.05

0.00

3. 58

6.58

0.22

500

509

710

$<50$

$<50$

$<50$

$<50$

$<50$

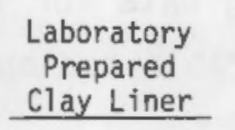

0.08

0.37

470

0.04

13.1

19.6

1370

9.3

5.7

130

770

4,400

0.06

0.03

0.02

0.16

0.06

0.50

0.01

0.08

ND

ND

ND

ND

ND

ND

ND

ND
Uncontacted

Foundation Soil

0.06

0.04

562

0.12

7.4

26.0

1610

5.1

4.6

450

1100

4850

0.1

0.04

0.02

0.03

0.03

0.00

0.01

0.08

ND

ND

ND

ND

ND

ND

ND

ND 
Extracts produced from each core subsample were chemically analyzed, and the averaged results for each depth are reported in Table 25. Radionuclide data were obtained by counting solid subsamples from Core No. 5 . Initial counting data for 1:1 extracts produced from Core No. 5 subsamples showed no measurable radionuclides in solution at any depth.

Results of the chemical analysis of the $1: 1$ extracts from the core subsamples indicated movement of $\mathrm{Cl}$ and $\mathrm{NO}_{3}$ through the entire liner system. These results were expected because the solubilities of $\mathrm{Cl}$ and $\mathrm{NO}_{3}$ are not limited in neutral $\mathrm{pH}$ conditions. Concentrations of $\mathrm{K}, \mathrm{Mg}$, and $\mathrm{Sr}$ were elevated in and below the clay liner, indicating that they were passing through the liner with little attenuation. While $\mathrm{Ca}, \mathrm{Na}$, and $\mathrm{SO}_{4} \mathrm{Con}^{-}$ centrations were high throughout the liner system, the source of these contaminants was in doubt due to their elevated concentrations in both the liner mix and the foundation soil. The clay liner did, in fact, attenuate As, Mo, Si, Se, and $V$ because clear reductions in concentrations can be seen below the clay liner depth. This was consistent with the leachate analysis collected from the moisture extraction cups and piezometers during the field tests. Radionuclide results also showed clear concentration reductions below the clay liner for all species measured.

The large variations in the reported results for extract analysis reflected the fact that the major form of attenuation provided by the clay liner in a neutral environment was adsorption by clay minerals. This form of attenuation has a finite ability to entrap contamination and its capabilities are enhanced by low leachate flow rates. Thus, variations in reported concentrations were most likely due to variations in the permeability of the liner system from location to location as shown in the core permeability measurements.

\section{CONCLUSIONS ON FIELD LINER EVALUATIONS}

Analysis of the moisture block and moisture extraction cup data thus far shows that the asphalt membrane and clay liner materials provided an effective contaminant barrier at the Grand Junction test pit. 
TABLE 25. Chemical Analysis of Core Subsamples: 1:1 Extract

\begin{tabular}{|c|c|c|c|c|c|}
\hline & $\begin{array}{c}\text { Column Top } \\
\text { to } \\
24.38 \mathrm{~cm} \\
\end{array}$ & $\begin{array}{l}24.38 \mathrm{~cm} \\
\text { to } \\
18.29 \mathrm{~cm}\end{array}$ & $\begin{array}{c}18.29 \mathrm{~cm} \\
\text { to } \\
12.19 \mathrm{~cm}\end{array}$ & $\begin{array}{l}12.19 \mathrm{~cm} \\
\text { to } \\
6.10 \mathrm{~cm}\end{array}$ & $\begin{array}{l}6.10 \mathrm{~cm} \\
\text { to } \\
\text { Bottom }\end{array}$ \\
\hline \multicolumn{6}{|c|}{ Macro ions (mg/L) } \\
\hline A1 & $0.05 \pm 0.06$ & $0.24 \pm 0.37$ & $0.02 \pm 0.03$ & $0.02 \pm 0.03$ & 0.00 \\
\hline B & $0.42 \pm 0.19$ & $0.27 \pm 0.21$ & $0.49 \pm 0.12$ & $0.45 \pm 0.007$ & $0.46 \pm 0.02$ \\
\hline $\mathrm{Ca}$ & $1029.3 \pm 110.03$ & $910.7 \pm 196.7$ & $1375.5 \pm 472.05$ & $1286.0 \pm 441.27$ & $1282.75 \pm 453.64$ \\
\hline $\mathrm{Fe}$ & $0.05 \pm 0.02$ & $0.06 \pm 0.004$ & $0.05 \pm 0.01$ & $0.11 \pm 0.12$ & $0.06 \pm 0.03$ \\
\hline K & $64.80 \pm 8.13$ & $72.91 \pm 2.91$ & $103.08 \pm 9.68$ & $111.66 \pm 31.23$ & $121.5 \pm 47.4$ \\
\hline Mg & $98.8 \pm 25.9$ & $151.3 \pm 44.1$ & $230.2 \pm 20.5$ & $242.1 \pm 69.5$ & $251.6 \pm 23.6$ \\
\hline $\mathrm{Na}$ & $935.0 \pm 217.3$ & $1108.5 \pm 367.3$ & $1393.1 \pm 392.6$ & $1089.3 \pm 282.9$ & $1209.6 \pm 101.6$ \\
\hline Si & $24.7 \pm 2.0$ & $18.1 \pm 7.0$ & $10.62 \pm 1.0$ & $7.9 \pm 1.7$ & $6.40 \pm 2.45$ \\
\hline$S r$ & $5.2 \pm 0.7$ & $5.5 \pm 1.2$ & $7.0 \pm 1.3$ & $6.2 \pm 1.2$ & $6.6 \pm 1.0$ \\
\hline $\mathrm{Cl}$ & $437 \pm 188$ & $551 \pm 384$ & $497 \pm 327$ & $500 \pm 217$ & $523 \pm 179$ \\
\hline $\mathrm{NO}_{3}$ & $350 \pm 184$ & $475 \pm 285$ & $506 \pm 310$ & $713 \pm 412$ & $896 \pm 525$ \\
\hline $\mathrm{SO}_{4}$ & $3367 \pm 153$ & $4383 \pm 1487$ & $4700 \pm 1345$ & $5333 \pm 757$ & $5300 \pm 819$ \\
\hline \multicolumn{6}{|c|}{ Trace Metals $(\mathrm{mg} / \mathrm{L})$} \\
\hline As & $0.55 \pm 0.42$ & $0.24 \pm 0.20$ & $0.06 \pm 0.03$ & $0.05 \pm 0.05$ & $0.08 \pm 0.06$ \\
\hline $\mathrm{Ba}$ & $0.04 \pm 0.01$ & $0.04 \pm 0.01$ & $0.06 \pm 0.01$ & $0.05 \pm 0.01$ & $0.03 \pm 0.03$ \\
\hline $\mathrm{Cr}$ & $0.03 \pm 0.01$ & $0.02 \pm 0.02$ & $0.04 \pm 0.02$ & $0.04 \pm 0.02$ & $0.04 \pm 0.02$ \\
\hline Mo & $1.53 \pm 0.03$ & $1.28 \pm 0.71$ & $0.74 \pm 0.48$ & $0.59+0.38$ & $0.16 \pm 0.13$ \\
\hline $\mathrm{Ni}$ & $0.06 \pm 0.01$ & $0.03 \pm 0.01$ & $0.02 \pm 0.04$ & $0.03 \pm 0.01$ & $0.03 \pm 0.01$ \\
\hline $\mathrm{Se}$ & $2.17 \pm 0.21$ & $1.34 \pm 0.33$ & $0.44 \pm 0.48$ & $1.03 \pm 0.58$ & $0.66 \pm 1.14$ \\
\hline$v$ & $2.41 \pm 0.12$ & $0.91 \pm 0.89$ & $0.02 \pm 0.01$ & $0.05 \pm 0.02$ & $0.04 \pm 0.02$ \\
\hline $2 n$ & $0.06 \pm 0.05$ & $0.04 \pm 0.04$ & $0.16 \pm 0.21$ & $0.04 \pm 0.02$ & $0.03 \pm 0.01$ \\
\hline \multicolumn{6}{|c|}{ Radionuclides $(\mathrm{pCi} / \mathrm{g})$} \\
\hline & 234 & 262 & $<50$ & $<50$ & $<50$ \\
\hline${ }^{210} \mathrm{~Pb}$ & 278 & 246 & $<50$ & $<50$ & $<50$ \\
\hline${ }^{226} \mathrm{Ra}$ & 361 & 359 & $<50$ & $<50$ & $<50$ \\
\hline $235_{U}$ & $<50$ & $<50$ & $<50$ & $<50$ & $<50$ \\
\hline
\end{tabular}


The moisture block data indicates that the asphalt membrane effectiveness was due to its impermeable characteristics. Inspection of asphalt samples showed them to be lively, of adequate thickness, with small crust formation on the exposed surface and very few surface cracks. Although a small zone of high moisture foundation soil was observed, it was attributed to faulty installation and inspection practices. A list of required construction practices is provided in this report.

The test clay liner, while allowing leachate to permeate through it, was effective in reducing concentrations of most cation and trace metal contaminants through adsorption to clay minerals. Anions in solution, including $\mathrm{Cl}$ and $\mathrm{NO}_{3}$, experience only limited attenuation while passing through the clay liner. These constituents will require specific treatment to ensure that their concentrations are below water quality guidelines. Radionuclide concentrations below the clay liner showed that no radionuclide movement away from the tailings pile was detected. It is probable that a more sensitive detection technique such as pulsed laser fluorimetry in neutral pH leachates would have confirmed that the clay liner was an effective radionuclide barrier.

The physical characteristics of the test clay liner indicated that it is possible to achieve a quality mixture of sodium bentonite and local soil in the field. Installation of the clay liner mixture under field conditions produced bulk density results ranging from 88 to $100 \%$ proctor for the samples tested. Because the hydraulic conductivity of the clay liner depends heavily on the bulk densitities achieved in the field, the most effective method for improving contaminant migration through a sodium bentonite liner is to ensure that maximum compaction is achieved uniformly over the entire area. 


\section{DECISION TREE FOR LINER EVALUATION}

The decision tree (Figure 37 ) is a logical sequence of questions to be answered and instructions to evaluate whether a particular site complies with the regulatory criteria for disposal of uranium mill tailings. It is intended to aid the firm(s) responsible for site design and remedial action of tailings piles exhibiting potential groundwater problems.

\section{SITE CHARACTERIZATIONS}

If the preliminary site assessment identifies a possible groundwater problem, decision tree methodology can be used. The first step in the procedure is site characterization. Because of the initial expense in using liners or having to take remedial action, comprehensive site characterization should be completed prior to construction activities so that the decision tree can be used to determine whether site-specific objectives can be met. This will save time and money if the design is inadequate to satisfy the criteria or if a more economical design that satisfies the criteria can be found.

It is possible (but unlikely) that sufficient data exist to describe the site for the purpose of the decision tree. This is the first decision point in the tree: Has the site been adequately characterized? If not, a site characterization plan should be devised to insure that all the necessary data will be obtained.

The decision on liner emplacement will ultimately be based on predictions made with state-of-the-art computer models; however, they can only be as good as the data obtained during site characterization. For this reason, the site characterization plan should be reviewed by an experienced hydrologist and geohydrochemist before execution. Site characterization data can be divided into three categories, which are discussed below:

1. site hydrology

2. tailings characterization

3. contaminant attenuation. 


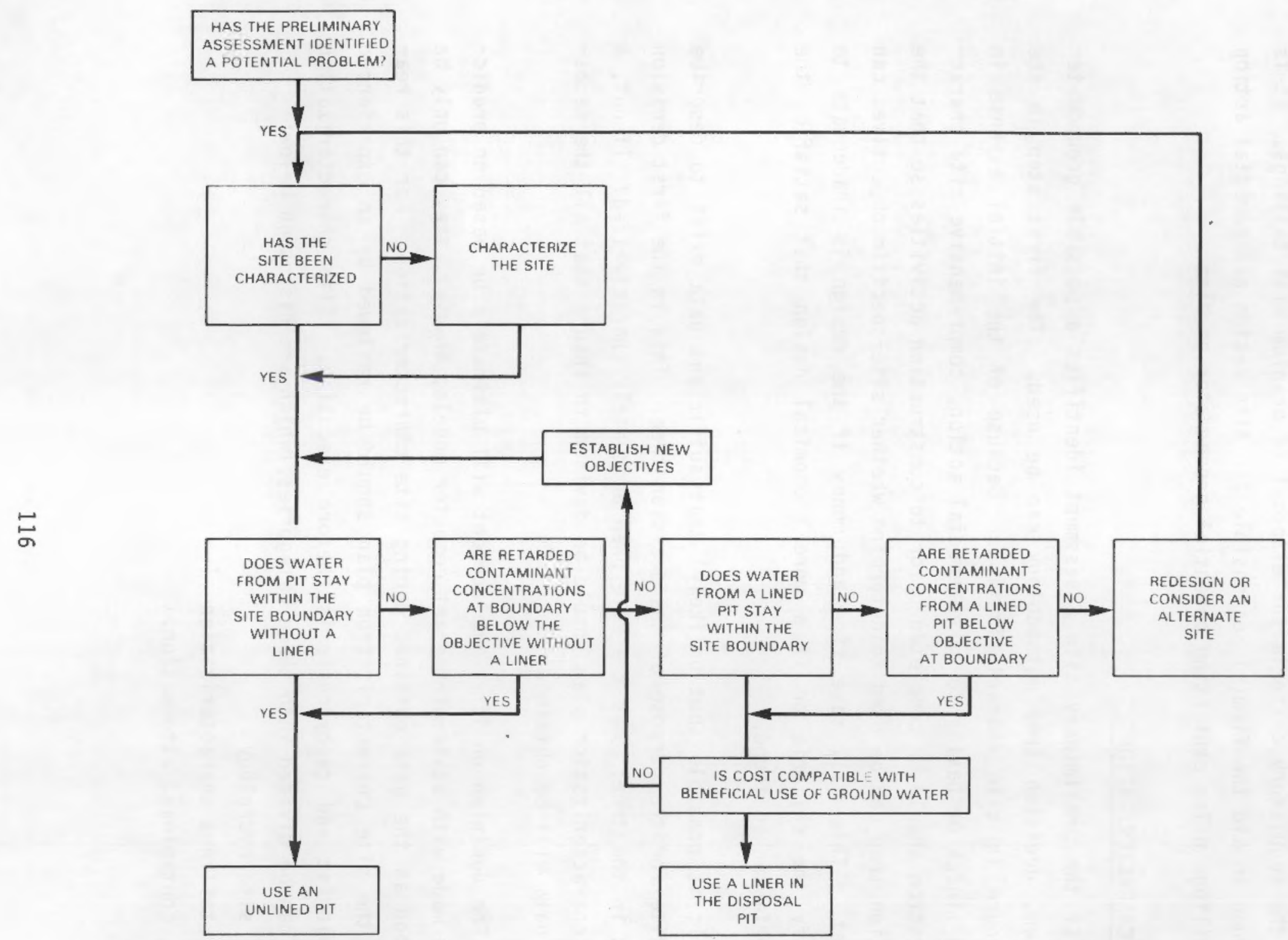

FIGURE 37. Decision Tree Evaluation of Inactive Uranium Processing Sites for Liner Requirements. (The use of hydrologic and solute transport computer codes is implicit in the Decision Tree.) 
Site Hydrology

Characterization of the site hydrology can be considered adequate if sufficient data exist to set up and run a hydrologic simulation that predicts the long-term behavior of water at the site. More than one type of model may be required for the simulation, depending on the particular site.

There are three sources of water that can remove contaminants: 1) infiltrating surface water; 2) subsurface or groundwater; and 3) water that exists initially in the tailings. All three sources should be characterized to predict the behavior of a disposal site.

Figure 38 shows the water balance for surface water at a hypothetical site. Precipitation and irrigation provide water to the surface, while runoff and evapotranspiration remove surface water. The remaining water is infiltration, which penetrates the cover layer of soil or fill. A layer of relatively impermeable material, such as a cover to inhibit radon emission, may divert some of the inflitrating water so that the net flux to the tailings is considerably lower than the infiltrating moisture. However, if radon is not considered a health hazard at a particular site such a cover may not be needed.

Generally, an unsaturated flow model is used to predict the flux of water entering the tailings pile. Such a model (UNSAT1D) was applied to a similar situation by Bond, Freshley, and Gee (1982). Climatic data(a) provide the time varying input (precipitation) to the UNSAT1D model as well as information needed to calculate evapotranspiration. Such data include daily temperature fluctuations, wind speed, cloud cover, and thunderstorm events (amount of rain, time of day, and duration).

\footnotetext{
(a) These data can be obtained from: U.S. Department of Commerce National Oceanic and Atmospheric Administration Environmental Data and Information Service National Climatic Center Federal Building Asheville, North Carolina 28801
} 


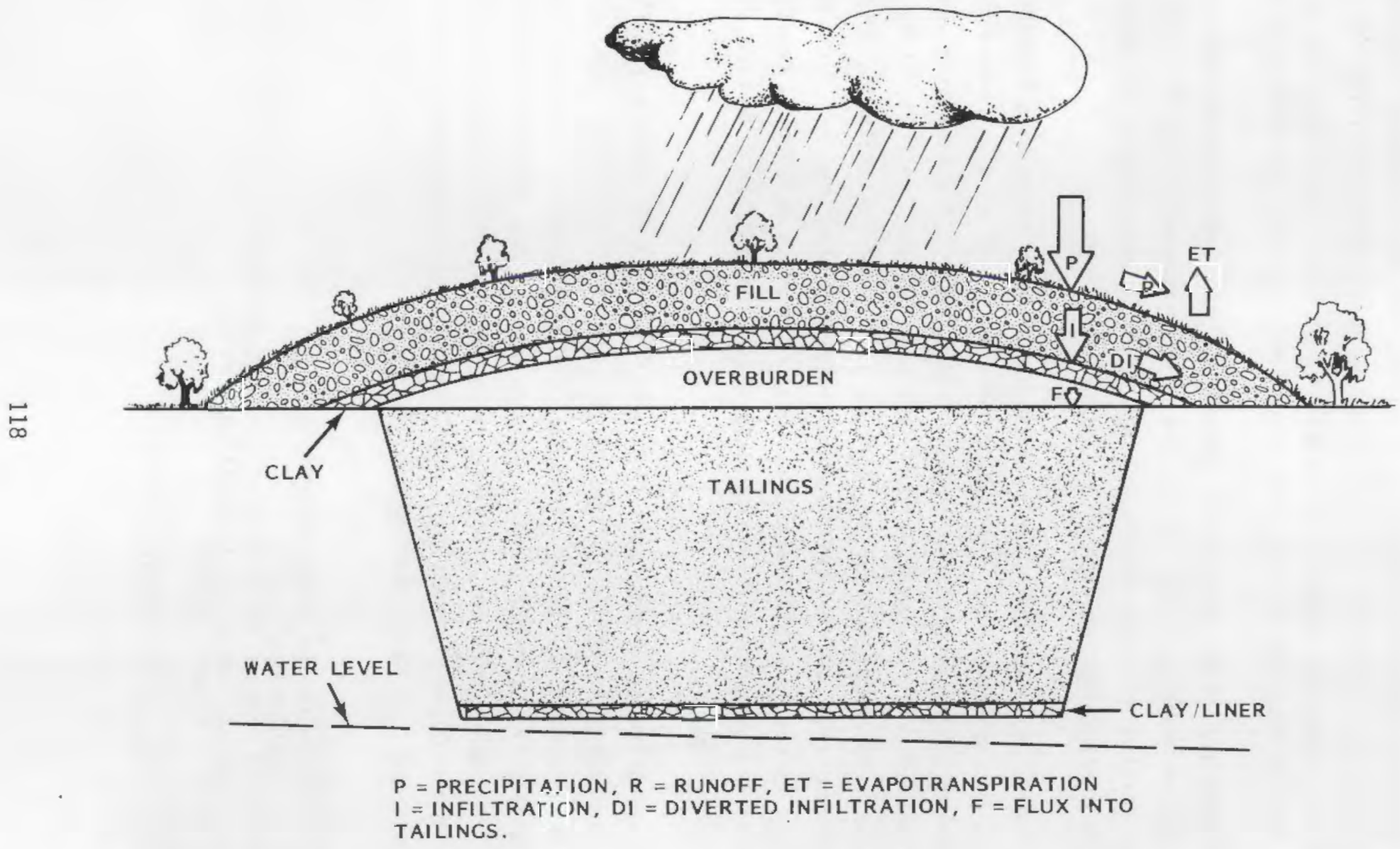

FIGURE 38. Water Balance for a Hypothetical Uranium Mill Tailings Disposal Site (Below Grade Example) 
Calculation of evaporation also requires data describing initial moisture content, porosity, bulk density, and depths of surface and underlying soil or radon barrier layers. Hydraulic conductivities of soils and barrier layers are required for unsaturated conditions (either unsaturated conductivities as a function of moisture potential or a saturated hydraulic conductivity along with a moisture retention curve). Finally, information on the type of vegetation and its water extraction behavior is needed.

For the decision tree, it must be assumed that the cover design has been completed and that the previously mentioned climate, vegetation, and soils data are available or can be obtained. A complete description of unsaturated flow models for calculating the moisture flux into and through the tailings (Bond et a 1. 1982) is beyond the scope of this document. An experienced hydrologist or soil physicist should be consulted to ensure that 1) the model used to calculate infiltration and flux to the tailings is satisfactory, and 2) the data required for model calculations are adequate.

Once the water flux above the tailings pile has been calculated, it can be used as an upper boundary condition for an unsaturated flow (or combination unsaturated and saturated flow) model of the tailings and subsurface hyrdology. The advantage of this approach is the difference in computational time steps required for a model that calculates evapotranspiration on a daily basis and a model that can use the infiltration/flux calculations on a monthly or yearly basis. The latter model can be run with longer time steps (and shorter run time). Flux calculations from UNSAT1D for an average or maximum rainfall year are input to a model that describes water transport through the tailings and subsurface layers. One model with this capability is TRUST (Reisenauer et al. 1982).

TRUST can be used to model subsurface water migration in one, two, or three dimensions. It can also be used to model combined unsaturated and saturated flow. Initial boundary conditions needed for this and other suitable models are similar. 
The following data are required for unsaturated subsurface hydrologic models:

- Tailings, soil, and liner hydraulic properties, including bulk density, porosity, saturated hydraulic conductivity, and a moisture retention curve or hydraulic conductivity as a function of moisture potential. These may change as leachate makes contact with the soil and/or liner; therefore, multiple sets of measurements under different conditions may be needed.

- Initial moisture content, the piezometric surface, distribution and the location of each layer (e.g., depth of top and bottom of the layer), and boundary conditions at the maximum depth (e.g., depth to water table or free drainage at maximum depth).

If saturated flow is also to be considered (depending on the specific site), the depths, thicknesses, and hydraulic conductivities of all layers must be known.

The data described in this section are all that are needed for the first test to determine whether or not a liner is required to satisfy design objectives: Does water from an unlined pit stay within the site boundary? However, to ensure that all questions regarding contaminant release from a tailings disposal site are answered, geochemical characterization of tailings and the chemical interactions between tailings leachate and soil/ liner materials and native ground water should be conducted simultaneously with site hydrologic characterization. This is logical from an economic standpoint as well because the subsurface and liner material samples needed for hydrologic characterization are also required to characterize leachate interactions with these materials.

Dne important interaction that is required for the hydrologic model is the behavior of liner and subsoil hydraulic conductivities as they are contacted with leachate. Gee et al. (1980) and Relyea et al. (1982) have measured hydraulic conductivities with tailings leachate in laboratory soil 
columns. Many of the saturated hydraulic conductivity values decrease as leachate passes through the column. Also, characterization of the inactive tailings may indicate that not all tailings require excavation and burial. Therefore, the next parameters are those that must be investigated to characterize tailings for the decision tree.

\section{Tailings Characterization}

Chemical compositions of uranium mill tailings may vary widely depending on the composition of the ore, the process used to extract uranium from the ore, and the history of the tailings since their disposal. These three factors largely determine the quantities and rates of contaminants released from tailings upon contact with water. Knowing the leach rate of contaminants from a tailings pile, however, will help in predicting the behavior of a tailings disposal site.

Various investigations have been conducted that attempt to determine the quantities of contaminants that will be released from tailings (Markos and Bush 1981a and b; Markos, Bush and Freeman 1981; and Buelt et al. 1981). These studies used batch or jar tests to obtain tailings leachate for chemical analysis. The batch method involves contacting a tailings sample with water in a container and shaking for a period of time. Afterwards, the solid and liquid are separated and the liquid is analyzed. Solid-to-solution ratios ranged from $1: 5$ to $1: 100$ ( $\mathrm{g}$ tailings $/ \mathrm{cm}^{3}$ water).

The batch method is acceptable qualitatively for screening a large number of tailings samples for relative contaminant concentrations, but it does not accurately determine how much contamination will be released from tailings and yields little (if any) information about contaminant release rates. Batch leach tests generally underestimate initial contaminant concentrations that may be leached from tailings upon contact with water.

The use of large columns $\left(2000 \mathrm{~cm}^{3}\right)$ allows analysis of radioactive and nonradioactive contaminants in the same sample with the precision needed to critically assess the first tailings leachate pore volume. Results indicate that effluent (leachate) from the tailings leach column should be 
collected in three or four equal fractions for analysis of the first pore volume. The second and third pore volumes should be collected in at least two fractions and thereafter sampled at least once per pore volume of leachate. The tailings characterization study showed that the contaminant concentrations fall rapidly within one or two pore volumes.

The contaminant source term generated by characterization of uranium mill tailings samples with the column leaching test is used as a boundary condition for contaminant concentrations at the tailings-soil or tailingsliner interface. Without data to describe the interactions of leachate with soil and liner material, it must be assumed that contaminants migrate unattenuated with the same velocity as water through subsurface layers. This assumption is clearly in error and can lead to the construction of expensive and potentially unnecessary engineered barriers. To make realistic predictions of attenuated contaminant migration, the interactions of tailings leachate with soil and liner material must also be quantified.

\section{Contaminant Attenuation}

To proceed with the decision tree to the point that predictions of retarded (or attenuated) contaminant concentrations at a site boundary can be compared with regulatory criteria, more data must be obtained for the hydrologic model. These data must somehow link the migration of contaminant to the predicted water movement, and this common link between solute (contaminant) and water transport is the retardation factor, which is discussed in more detail by Relyea, Serne and Rai (1980) and Relyea (1982).

Generally, the retardation factor is valid under very restricted, ideal conditions where only adsorption is considered. A discussion of recent investigations of solute retardation under less than ideal conditions in saturated porous media is given by Nkedi-Kizza et al. (1982), but the only mechanism for solute retardation in this transport model is ideal adsorption (e.g., linear adsorption isotherm). A solute transport model for retardation by nonlinear adsorption in saturated and unsaturated porous media is given by Persaud and Wierenga (1982). 
Neither of these two transport models (or any other model) is presently able to predict solute/contaminant transport adequately under the conditions found at a uranium mill tailings disposal site. The reason for this is that state-of-the-art solute transport models are not yet capable of describing contaminant attenuation caused by precipitation, contaminant scavenging by coprecipitation, and nonideal adsorption. This limitation results in overly conservative predictions since most contaminant attenuation from acidic tailings piles is apparently a result of $\mathrm{pH}$-induced precipitation reactions (Gee et al. 1980, Markos and Bush 1981b).

Fortunately, contaminant precipitation from tailings leachate is very dependent on $\mathrm{pH}$, which can be predicted as the leachate moves through subsurface soil and liner material (Nelson, Reisnauer, and Gee 1980). To predict contaminant migration, two retardation factors are required for each contaminant: one retardation factor that describes contaminant migration in unneutralized (1ow $\mathrm{pH}$ ) leachate, and another that describes contaminant migration through soil or liner in neutralized (higher $\mathrm{pH}$ ) leachate. Two retardation factors are needed for each contaminant in each layer of soil or liner material that contacts the leachate. A retardation factor for the $\mathrm{pH}$ is also needed. Methods for obtaining the required data are discussed below.

\section{pH Retardation Factor}

The retardation factor $R_{f}$ for an ideally adsorbed contaminant can be expressed by

$$
R_{f}=1+\frac{\rho^{b}}{\theta} R d
$$

where $\quad b=$ bulk density of the porous media

$$
\begin{aligned}
\theta= & \text { the volumetric water content of the porous media (under } \\
& \text { saturated conditions } \theta \text { is equal to the porosity } \epsilon \text { ) } \\
\text { Rd = } & \text { the adsorption coefficient (or distribution ratio) for the } \\
& \text { contaminant }
\end{aligned}
$$


The distribution ratio, $R d$, is defined by

$$
R d=\frac{\text { Amount of contaminant adsorbed on the solid }(\mathrm{meq} / \mathrm{g})}{\text { Amount of contaminant in solution }\left(\mathrm{meq} / \mathrm{cm}^{3}\right)}
$$

The Rd (or $\mathrm{Kd}$, as it is sometimes referred to) can be measured using either batch or column techniques (Relyea et al. 1980, Relyea 1982). The approach of Nelson et al. (1980) will be used here to define an Rd value for the special case of $\mathrm{pH}$.

The titration curve, shown in Figure 23, illustrates the change in the pH of a Clive, Utah, soil as leachate from Shiprock tailings was added. This study and those of Gee et al. (1980) have shown that many of the contaminant concentrations are greatly reduced at $\mathrm{pH} 7.7$; therefore, Rd will be calculated for the $\mathrm{pH}$ at $\mathrm{pH}=7.7$.

Acidity (high $\mathrm{H}^{+}$concentrations) may be thought of as a contaminant because highly acidic conditions increase solubilities and, therefore, concentrations of many other contaminants. The total acidity for leachate is given by the sum of $\mathrm{H}^{+}+\mathrm{Al}^{+3}+\mathrm{Fe}^{+3}$ concentrations in meq per liter. In addition to $\mathrm{H}^{+}$, the total acidity includes $\mathrm{Al}^{+3}$ and $\mathrm{Fe}^{+3}$ because at higher $\mathrm{pH}$ values these hydrous oxides are precipitated and release hydrogen ions equivalent to the original $\mathrm{Al}$ and $\mathrm{Fe}$ concentrations. From Table 13 the total acidity is found to be $300 \mathrm{meq} / \mathrm{L}$. At $\mathrm{pH} 7.7$ this soil neutralizes or adsorbs 230 meq total acidity per 100 grams (see Figure 33 ). The resulting $\mathrm{Rd}$ for Shiprock leachate at $\mathrm{pH}=7.7$ in this soil is

$$
R d=\frac{230 \mathrm{meq} / 100 \mathrm{~g}_{3}}{300 \mathrm{meq} / 1000 \mathrm{~cm}}=7.7 \mathrm{~cm}^{3} / \mathrm{g} .
$$

The Rd obtained implies that $1 \mathrm{~g}$ of this soil will neutralize $7.7 \mathrm{~cm}^{3}$ of Shiprock leachate to a pH of 7.7.

If $\mathrm{pH}=4.5$ had been selected for the above example, the result would have shown that 100 grams of soil neutralizes 470 meq of acidity. The Rd value for $\mathrm{pH}=4.5$ is 


$$
\mathrm{Rd}=\frac{470 \mathrm{meq} / 100 \mathrm{~g}}{300 \mathrm{meq} / 1000 \mathrm{~cm}}=15.7 \mathrm{~cm}^{3} / \mathrm{g} .
$$

One gram of soil will neutralize $15.7 \mathrm{~cm}$ of Shiprock leachate to $\mathrm{pH} 4.5$.

The retardation factor for this soil can be found from Equation (7). If the soil bulk density is $1.5 \mathrm{mg} / \mathrm{cm}^{3}$ and porosity is 0.4 , the $\mathrm{pH} 7.7$ retardation factor is

$$
R_{f}=1+\frac{p^{b}}{\theta} R d
$$

or

$$
R_{f}=1+\frac{1.5}{0.4}(7.7)=30
$$

In other words, the Shiprock leachate $\mathrm{pH}$ front (at pH 7.7) moves 30 times slower than water under saturated conditions.

The $\mathrm{pH}$ retardation factor at $\mathrm{pH}=4.5$ is

$$
R_{f}=1+\frac{1.5}{0.4}(14.7)=56 \text {. }
$$

The Shiprock leachate pH front at pH 4.5 moves 56 times slower than water.

\section{Contaminant Retardation Factors}

To predict contaminant transport, two Rd values are needed for each contaminant. For this example, one Rd value is needed at pH 7.7 and another is needed for the contaminant in unneutralized leachate. Gee et al. (1980) used a batch method to calculate Rd values.

A known volume of leachate (V) was added to a known amount of soil $(W)$, and contaminant concentrations were measured before contact $\left(C_{0}\right)$ and afterward $\left(C_{f}\right)$. The amount of contaminant adsorbed on or precipitated in 
the soil is $\left(C_{0}-C_{f}\right) V$; therefore, the contaminant concentration per gram of soil is $\left(C_{0}-C_{f}\right) V / W$. The $R d$ is defined by the ratio of the contaminant concentration per gram of soil to the final contaminant concentration in the leachate:

$$
R d=\frac{\left(c_{0} y-c_{f}\right) / w}{c_{f}}
$$

or more simply,

$$
\operatorname{Rd}=\frac{v}{W}\left(\frac{C_{0}}{C_{f}}-1\right)
$$

Two Rd values are required for each contaminant (one for unneutralized and one for neutralized tailings leachate). An Rd value is also needed to describe movement of the pH front. If alkaline or neutral tailings are leached, precipitation may not be a major retardation mechanism. In these cases, only one Rd value may be required (for each contaminant). Once the site has been characterized, the first box in the decision tree is complete, and we proceed to the second decision point.

\section{WATER MOVEMENT WITHOUT A LINER}

This step requires an experienced groundwater hydrologist who is able to interpret and utilize the site characterization data to set up a water transport model that describes the disposal site. This step is far from trivial, but details are beyond the scope of this document. Readers are referred to Simmons and Gee (1981), Reisenauer et al. (1982), Nelson et al. (1980a), and Bond et a1. (1982) for more detailed information on setting up and running computer simulations of water transport.

Once the computer model or models have been set up and run, the question is: Does water from the tailings stay within the site boundary during 
the control period as defined by state and federal regulations? If water does stay within the site boundary for an unlined disposal area, contaminants will also remain within the boundary. However, the computer model is already set up at this point and contaminant transport can be predicted for one-fourth to one-third the cost of setting up and running the water transport simulation. Although a prediction of contaminant transport may not be required, it may be very useful as additional evidence that a site without a liner more than satisfies the regulatory criteria. If water from the unlined tailings pit is found to move beyond the site boundary, contaminant transport must be predicted.

\section{CONTAMINANT TRANSPORT WITHOUT A LINER}

At this point, water is predicted to move from the tailings to beyond the site boundary at some time less than a specified control period. The next step is to predict contaminant migration using the tailings leachate data and contaminant retardation data gathered during site characterization. The question is: Do contaminant concentrations from the tailings stay below the limit set by the disposal site design objectives at or beyond the site boundary for an unlined disposal area? If the answer is yes, no liner is required for tailings disposal at that site. If the answer is no, contaminant levels above the limits are predicted to cross the boundary for an unlined tailings pile; thus, either a new disposal design must be formulated with the possible inclusion of a liner, or new design objectives must be established in conformance with regulatory agencies.

\section{WATER MOVEMENT WITH A LINER}

The water transport model for the site may be altered to include a liner at this point. Liner thickness and hydraulic conductivity are added to the water transport model, and a new computer simulation of water movement with the liner must be run and compared with the design objectives. The question now is: Does water from a lined tailings disposal area stay within the site boundary in the control period specified by the design objectives agreed to by state and federal agencies? If the answer is yes, 
a lined tailings disposal area satisfies the objectives and may be used. However, the cost of a liner system must be affordable in relation to the beneficial use of the groundwater described by the design objectives. Again, the prediction of contaminant migration may be useful as additional evidence that the site design is satisfactory. If the answer is no, water from tailings is predicted to cross the boundary and the movement of contaminants must be considered.

Various liner materials may be evaluated at this point. Barnes, Buelt, and Hale (1981) have published data on the performance of catalytic airblown asphalt membranes. Recent data indicate permeabilities in the range of $10^{-11}$ to $10^{-12} \mathrm{~m} / \mathrm{s}$. Sodium bentonite amended soils may also be used for low permeability liner material.

\section{CONTAMINANT TRANSPORT WITH A LINER}

Contaminant attenuation data (contaminant $\mathrm{Rd}$ values, Rd value for the selected $\mathrm{pH}$ front) for the liner and subsurface soils must be added to the water movement model for a lined disposal site. Another computer simulation is required to answer the question: Do contaminant concentrations transported from a lined tailings disposal area stay within the site boundary during the control period? If contaminant concentrations at the site boundary meet the objectives during the control period, a lined tailings disposal site can be used. Again, the cost of the liner system must be taken into consideration. If the objectives are not met, predicted contaminant releases are excessive and the site should be redesigned (by using a thicker liner, different liner material, or a new approach) or an alternative site considered. Both of these options require that the decision tree process be repeated from the start. If the same site is to be used with a new design, however, much of the characterization is already completed.

\section{DEMONSTRATION}

Relyea (1983) has demonstrated the decision tree for a hypothetical disposal site. The demonstration also showed that infiltration through the 
cover is difficult unless composed of porous sand or rock that reduces evapotranspiration. Therefore, infiltration at Western U.S. sites may not be likely and the only water source may be from movement of a shallow groundwater table. Nevertheless, the demonstration, using the UNSATID and TRUST codes, compared natural soil and liners as contaminant retardation barriers for a variety of configurations and leachates. It showed that the decision tree is a logical sequence that can be helpful in determining liner requirements for a mill tailings impoundment. 



\section{REFERENCES}

ASTM. 1978. Standard Method for Moisture-Density Relation of Soils and Soil-Aggregate Mixtures Using 1D-1b Rammer and 18-in Drop. (D1557-78). Part 19:278-284.

The Asphalt Institute. 1976. Asphalt in Hydraulics. Manual Series No. 12, College Park, Maryland.

Barnes, S. M., J. L. Buelt and V. Q. Hale. 1981. Accelerated Aging Tests of Liners for Uranium Mill Tailings Disposal. PNL-4049, Pacific Northwest Laboratory, Richland, Washington.

Blodgett, R. B., and R. G. Fisher. 1969. Insulations and Jackets for Control and Power Cable in Thermal Reactor Nuclear Generating Stations. okonite Company, Institute of Electric and Electronic Engineers, Passaic, New Jersey.

Blokker, P. C., and H. van Hoorn. 1959. "Durability of Bitumen in Theory and Practice." Fifth World Petroleum Congress, Section VI, Paper 27, Pp. 417-432. New York, New York.

Bond, F. W., M. D. Freshley and G. W. Gee. 1982. Unsaturated Flow Modeling of a Retorted 0 il Shale Pile. PNL-4248, Pacific Northwest Laboratory, Richland, Washington.

Buelt, J. L., V. Q. Hale, S. M. Barnes and D. J. Silviera. 1981. An Evaluation of Liners for a Uranium Mill Tailings Disposal Site - A Status Report. D0E/UMT-0200, Pacific Northwest Laboratory, Richland, Washington.

Cass, A., and M. E. Sumner. 1982. "Soil Pore Structure and Irrigation Water Quality: I. Empirical Sodium Stability Model." Soil Sci. Soc. Am. J. $46: 503-506$.

Clark, D. A. et al. 1974. An Evaluation of Tailings Pond Sealants. EPA-660/2-74-065. U.S. Environmental Protection Agency, Corvallis, Oregon. Report PB 235929, National Technical Information Service, Springfield, Virginia.

Dunning, R. L. 1975. "Rubber in Asphalt Emulsion Systems and Their Rheological 8ehavior." Paper presented at the Second Annual Meeting of the Asphalt Emulsion Manufacturers Association, March 5, 1975, San Diego, California.

Edwald, G. W. 1973. "Stretching the Lifespan of Synthetic Pond-Linings." Chemical Engineering. October 1, 1973:67-70.

E. I. du Pont de Nemours and Co. A. "Engineering Guide to the du Pont Elastomers." Technical Bulletin, E-26276, E. I. du Pont de Nemours and Co., Inc., Elastomer Chemicals Department, Wilmington, Delaware. 
E. I. du Pont de Nemours and Co. B. "Excerpts from a du Pont Seminar on Flexible Pond and Reservoir Liners and Covers." E. I. du Pont de Nemours and Co., Inc., Elastomer Chemicals Department, Wilmington, Delaware.

E. I. du Pont de Nemours and Co. C. "Flexible Membranes for Pond and Reservoir Liners and Covers." Technical Bulletin, E-23335, E. I. du Pont de Nemours and Co., Inc., Elastomer Chemicals Department, Wilmington, Delaware.

E. I. du Pont de Nemours and Co. D. "Radiation Resistance of Elastomers and Plastics." E. I. du Pont de Nemours and Co., Inc., Elastomer Chemicals Department, Wilmington, Delaware.

Foster, A. R., and R. L. Wright, Jr. 1973. Basic Nuclear Engineering. Allyn and Bacon, Inc., Boston, Massachusetts.

Frobel, R. K., et al. 1977. Development of a Low-Cost Aspha1t-Rubber Membrane for Water Harvesting Catchments and Reservoir Seepage Control. University of Arizona, Tucson, Arizona. Report PB 268779, National Technical Information Service, Springfield, Virginia.

Fuller, W. H. 1981. Liners of Natural Porous Materials to Minimize Pollutant Higration. EPA-600/2-81-122, U.S. Environmental Protection Agency, Cincinnati, Ohio.

Gee, G. W., A. C. Campbell, 8. E. Opitz and D. R. Sherwood. 1980. "Interaction of Uranium Mill Tailings Leachage with Morton Ranch Clay Liner and Soil Material." In Uranium Mill Tailings Management, Proceedings of the Third Symposium, pp. 333-352. Geotechnical Engineering Program, Colorado State University, Ft. Collins, Colorado.

Geir, F. H., and W. R. Morrison. 1968. Buried Asphalt Membrane Canal Lining. U.S. Department of Interior Research Report No. 12. Washington, D.C.

Griffin, R. A., and N. F. Shimp. 1978. Attenuation of Pollutants in Municipal Landfill Leachate by Clay Minera1s. EPA-600/2-78-157, U.S. EPA Municipal Environmental Research Laboratory, Cincinnati, Ohio.

Hartley, J. N., et al. 1980. Annual Report on the Asphalt Emulsion Sealing of Uranium Mill Tailings. PNL-3752, DOE/UMT-0201, Pacific Northwest Laboratory, Richland, Washington.

Haxo, H. E., Jr. 1976a. "Assessing Synthetic and Admixed Materials for Lining Landfills." In Gas and Leachate from Landfills, Formation, Collection and Treatment, eds. E. J. Genetelli and J. Circello, pp. 130-158. Report EPA 600/9-76-004, U.S. Environmental Protection Agency, Cincinnati, Ohio. Report P8 251161, National Technical Information Service, Springfield, Virginia. 
Haxo, H. E., Jr., and R. M. White. 1976a. Evaluation of Liner Materials Exposed to Leachate. Matrecon, Inc., Oakland, California. Report PB 259913, National Technical Information Service, Springfield, Virginia.

Haxo, H. E., Jr., and R. M. White. 1976b. Evaluation of Liner Materials Exposed to Leachate, Second Interim Report. EPA-600/2-76-255, National Technical Information Service, Springfield, Virginia.

Haxo, H. E., Jr., et al. 1977. Liner Materials Exposed to Hazardous and Toxic Sludges. Matrecon, Inc., Oakland, California, Report PB 271013, National Technical Information Service, Springfield, Virginia.

Haxo, H. E., Jr. et al. 1979. Liner Materials Exposed to Municipal Solid Waste Leachate. Matecon, Inc., Oakland, California. Report PB 299336, National Technical Information Service, Springfield, Virginia.

Hickey, K. B. 1967. Concrete and Epoxy Materials Compared; Load Properties, Durability, and Volume Change. Report C-1313, U.S. Department of the Interior, Bureau of Reclamation, Denver, Colorado. Report PB 183361, National Technical Information Service, Springfield, Virginia.

Hickle, R. D. 1976. "Impermeable Asphalt Concrete Pond Liner." Civil Engineering August 1976:56-59.

Hille1, D. 1980. Fundamentals of Soil Physics. Academic Press, Inc., New York, New York.

Hoff, G. C. et a1. 1977. Identification of Candidate Zero Maintenance Paving Materials, Volume 2. U.S. Army Engineer Waterways Experiment Station, Concrete Laboratory, Vicksburg, Mississippi. Report AD A053794, National Technical Information Service, Springfield, Virginia.

Hughes, J. 1974. "Use of Wyoming Bentonite as a Soil Sealant for the Impoundment of Highly Contaminated Industrial Wastes." 29 th Annual Industrial Waste Conference. Purdue University, West Lafayette, Indiana.

Levenspiel, 0. 1972. Chemical Reaction Engineering. John Wiley and Sons, Inc., New York, New York.

Lindsay, W. L. 1979. Chemical Equilibria in Soils, John Wiley and Sons, New York, New York.

Lubina, R. H. et al. 1979. "Design and Construction of the Lining System for the Cotter Tailings Impoundment." In Symposium on Uranium Mill Tailings Management, 13-26, Fort Collins, Colorado, November 19-20, 1979. Colorado State University, Civil Engineering Department, Geotechnical Engineering Program, Fort Collins, Colorado. 
Markos, G., and K. J. Bush. 1981a. Contamination of Ground and Surface Waters by Uranium Mining and Milling, Volume II. Field Sampling and Empirical Modeling. Work performed for U.S. Bureau of Mines by Geochemistry and Environmental Chemistry Research Inc., Rapid City, South Dakota.

Markos, G., and K. J. Bush. 1981b. Geochemical Investigation of UMTRAP Designated Site at Salt Lake City, Utah. UMTRA-DOE/AL0-227, Geochemistry and Environmental Chemistry Research Inc., Rapid City, South Dakota.

Markos, G., K. J. Bush and T. Freeman. 1981. Geochemical Investigation of UMTRAP Designated Site at Canonsburg, Pennsyl vania. UMTRA-DOE/AL0-226.

Mattia, R. F. and D. R. Luh. 1971. "Radiation Resistance of EPOM, Polychloroprene, and Chlorosulfonated Polyethylene." Presented at the 1971 Tri-State Regional Meeting of the Massachusetts, Connecticut, and Rhode Island Rubber Groups, May 13, 1971, Auburn, Massachusetts.

Middlebrooks, E. J. et al. 1978. Waste Water Stabilization Pond Linings. Special Report 78-28, Department of the Army, Cold Regions Research and Engineering Laboratory, Corps of Engineers, Hanover, New Hampshire, Report AD AD629903, National Technical Information Service, Springfield, Virginia.

Nelson, R. W., G. W. Gee and C. A. Oster. 1980. Radon Control by Multilayer Earth Barriers. 1. Modeling of Moisture and Density Effects on Radon Diffusion from Uranium Mill Tailings. In Uranium Mill Tailings Management Proceedings of the Third Symposium. pp. 79-88. Geotechnical Engineering Program, Colorado State University, Ft. Collins, Colorado.

Nelson, R. W., A. E. Reisenauer and G. W. Gee. 1980. Model Assessment of Alternatives for Reducing Seepage from Buried Uranium Mill Tailings at the Morton Ranch Site in Central Wyoming. PNL-3378, Pacific Northwest Laboratory, Richland, Washington.

Nkedi-Kizza, P., P.S.C. Rao, R. E. Jessup and J. M. Davidson. 1982. "Ion Exchange and Diffusive Mass Transfer During Miscible Displacement Through an Aggregated 0xisol." Soil Sci. Soc. Am. J. 46:471-476.

Persaud, N., and P. J. Wierenga. 1982. "A Differential Model for One-Dimensionsal Cation Transport in Discrete Homoionic Ion Exchange Media." Soil Sci. Soc. Am. J. 46:482-490.

Reisenauer, A. E., K. T. Key, T. N. Narasimhan and R. W. Nelson. 1982. TRUST: A Computer Program for Variably Saturated Flow in Multidimensional, Deformable Media. NUREG/CR-2360, Pacific Northwest Laboratory, Richland, Washington. 
Relyea, J. F. 1983. A Decision Tree Approach to Evaluating Inactive Uranium Processing Sites for Liner Requirements. PNL-4436, Pacific Northwest Laboratory, Richland, Washington.

Relyea, J. F., and W. J. Martin. 1982. "Evaluation of Inactive Uranium Mill Tailings Sites for Liner Requirements: Characterization and Interaction of Tailings, Soil, and Liner Materials." Uranium Mill Tailings Management, pp. 507-519. Colorado State University, Ft. Collins, Colorado.

Relyea, J. F., R. J. Serne and D. Rai. 1980. Methods for Determining Retardation Factors: Status Report. PNL-3349, Pacific Northwest Laboratory, Richland, Washington.

Relyea, J. F., W. J. Martin and D. T. Colclough. 1982. "Long-Term Performance Testing." In Nuclear Waste Management Seminannual Progress Report, October 1981 through March 1982. eds. T.D. Chikalla, and J.A. Powe11, PNL-4250-1, Pacific Northwest Laboratory, Richland, Washington.

Relyea, J. F. 1982. "Theoretical and Experimental Considerations for the Use of the Column Method for Determining Retardation Factors." Radioactive Waste Mgt. 3:115-130.

Rollins, M. B. and A. S. Dylla. 1970. "Bentonite Sealing Methods Compared in the Field." Journal of Irrigation and Drainage Division, ASCE 96(IR2):193-203.

Savartnick, H. A. 1969. Polyvinyl Chloride. Robert E. Krieger Publishing Co., Huntington, New York.

Serne, R. J., S. R. Peterson, and G. W. Gee. 1983. Laboratory Measurement of Contaminant Attenuation of Uranium Mill Tailings Leachates by Sediments and Clay Liners. NUREG/CR-3124 (PNL-4605), Pacific Nor thwest Laboratory, Richland, Washington.

Sherard, J. L., L. P. Dunnigan and R. S. Decker. 1976. "Identification and Nature of Dispersive Soils," Journal of the Geotechnical Engineering Division, ASCE, Vol. 102, No. GT4, Pro. Paper 12052, April 1976, pp. 287-301.

Sherwood, D. R. and R. J. Serne. 1983. Evaluation of Selected Neutralizing Agents for the Treatment of Uranium Tailings Leachates: Laboratory Progress Report, NUREG/CR-3030 (PNL-4524), Pacific Northwest Laboratory, Richland, Washington.

Shultz, D. W. and M. P. Miklas, Jr. 1980. "Assessment of Liner Installation Procedures." In: Disposal of Hazardous Wastes. D. Shultz, ed. U.S. Environmental Protection Agency, Municipal Environmental Research Laboratory Report EPA 600/9-80-010, Cincinnati, Ohio. Report PB 80 175086, National Technical Information Service, Springfield, Virgina. 
Simmons, C. S., and G. W. Gee. 1981. Simulation of Water Flow and Retention in Earthen Cover Materials Overlying Uranium Mill Tailings. PNL-3877, Pacific Nor thwest Laboratory, Richland, Washington.

Stewart, W. S. 1978. State-of-the-Art Study of Land Impoundment Techniques. Report EPA 600/2-78-196, U.S. Environmental Protection Agency, Cincinnati, Ohio. Report PB 291881, National Technical Information Service, Springfield, Virginia.

Tamura, T. and W. J. Boegley, Jr. 1983. In Situ Grouting of Uranium Mill Tailings Piles: An Assessment. ORNL/TM-8539, Oak Ridge NationaT Laboratory, Oak Ridge, Tennessee.

Tyson, S. S. 1978. Internally Sealed Concrete for Bridge Deck Protection. Report PB 287803, National Technical Information Service, Springfield, Virginia.

U.S. Environmental Protection Agency (USEPA). National Interim Primary Drinking Water Regulations. 40 CFR 141, Federal Register, Washington, D.C.

U.S. Environmental ProtectionAgency (USEPA). 1983. Standards for Remedial Actions at Inactive Urnaium Processing Sites. 40 CFR 192, Federal Register, Washington, D.C.

U.S. Nuclear Regulatory Commission (NRC). 1979. Draft Generic Impact Statement on Uranium Milling. NUREG-0511, 2 volumes, Washington, DC.

U.S. Nuclear Regulatory Commission (NRC). 1980. Final Environmental Statement Related to Western Nuclear, Inc. NUREG-0639, Nuclear Regulatory Commission, Washington, D.C.

Van Asbeck, W. F. 1964. Bitumen in Hydraulic Engineering. Elsevier Publishing Co., New York, New York.

Van Oort, W. P. 1956. "Durability of Asphalt - Its Aging in the Dark." Industrial and Engineering Chemistry 58:1196-1201.

Williams, R. E. 1978. "Control and Prevention of Seepage from Uranium Mill Waste Disposal Facilities." Proceedings of the Nuclear Energy Agency Seminar on Management, Stabilization and Environmental Impact of Uranium Mill Tailings, July 1978, Albuquerque, New Mexico. 
APPENDIX A

PERMEABILITY TEST DATA 
TABLE A.1. Laboratory Permeability Testing Data

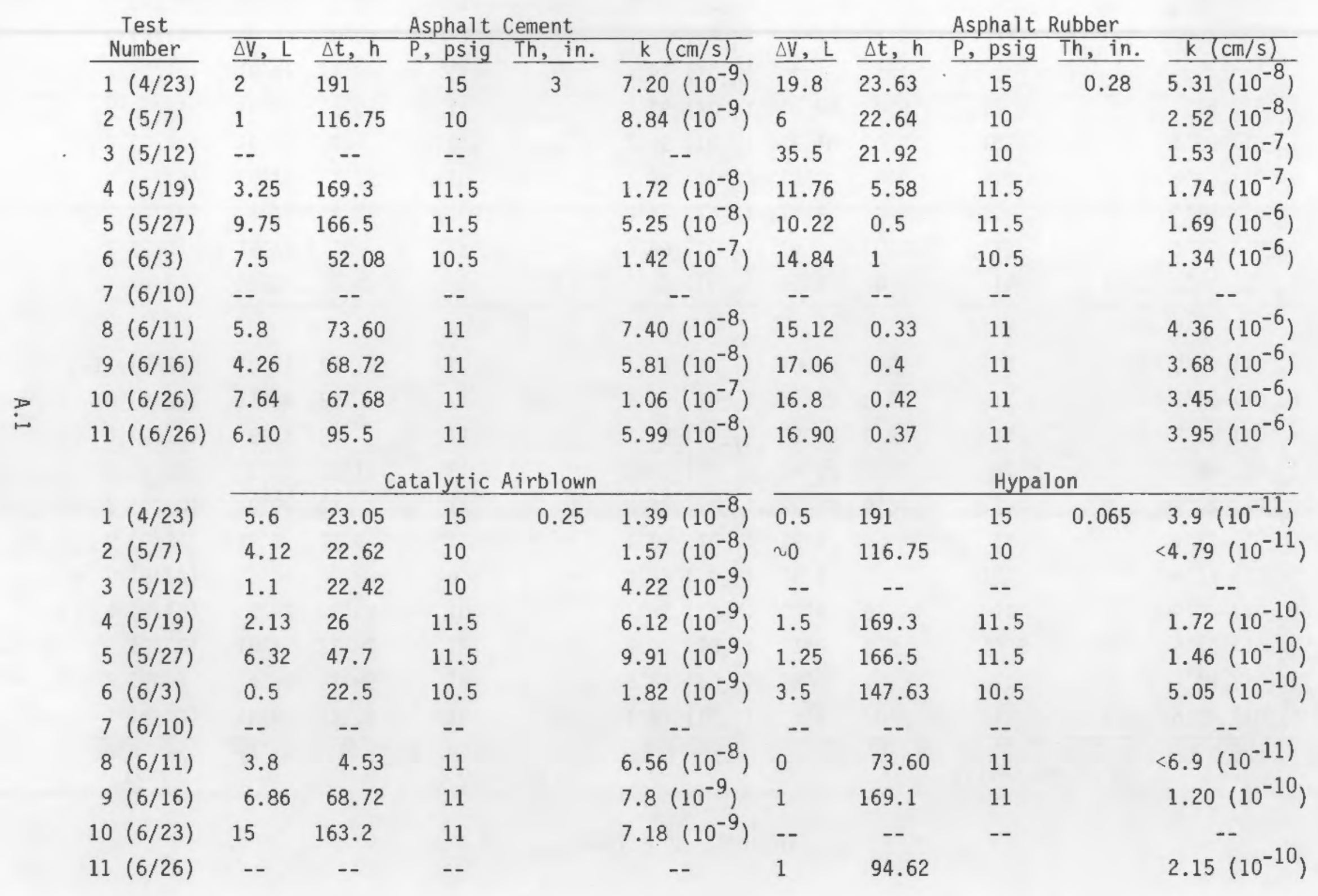


TABLE A.1. (Contd)

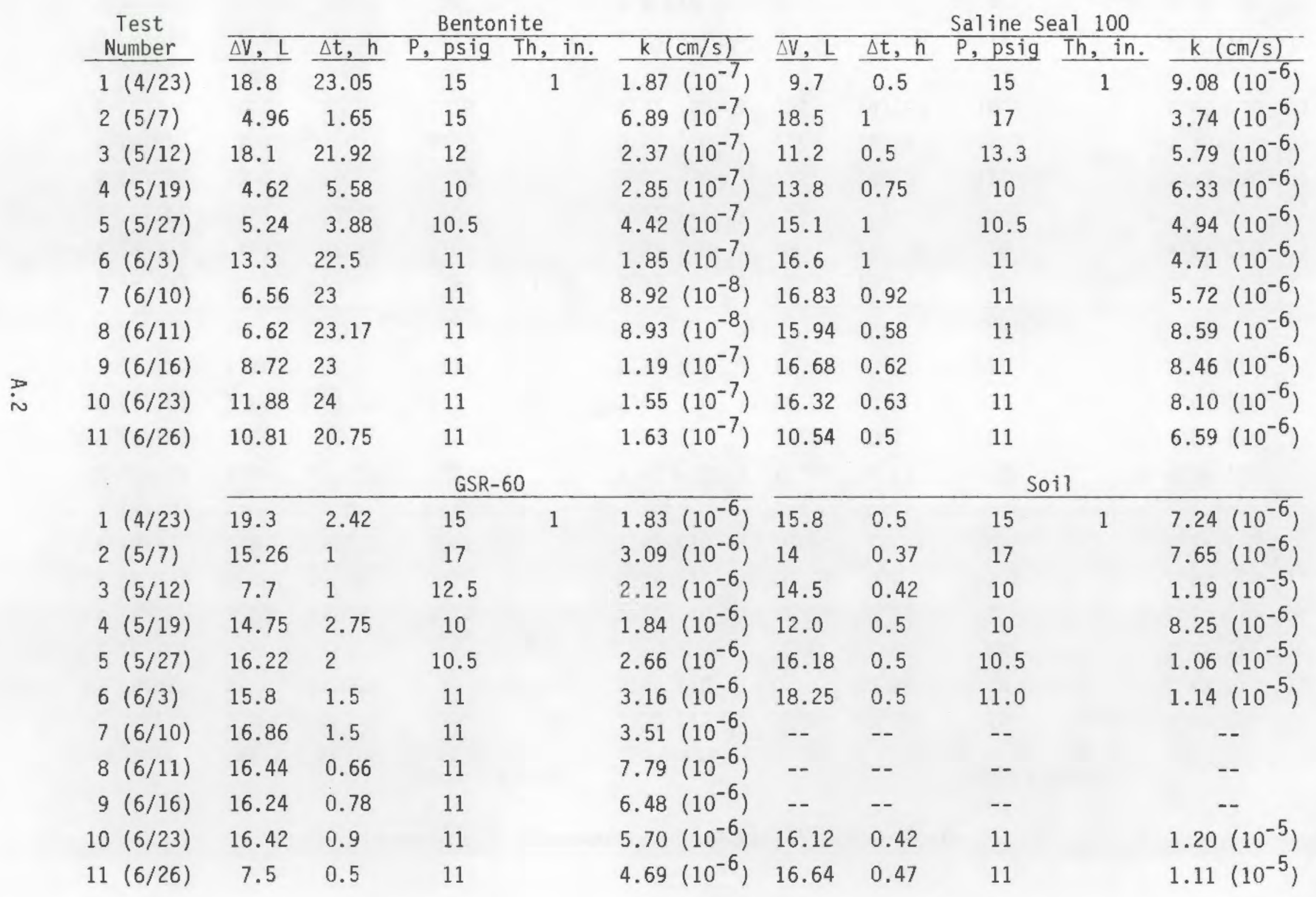




\section{APPENDIX B}

ASPHALT LINER SPECIFICATIONS 


\section{ASPHALT LINER SPECIFICATIONS}

TABLE B.1. AR4000 Asphalt Cement Specifications

\begin{tabular}{|c|c|c|c|}
\hline $\begin{array}{c}\text { Property } \\
\end{array}$ & Minimum & Maximum & Actual \\
\hline \multicolumn{4}{|l|}{ Tests on original asphalt: } \\
\hline Flash point, $\mathrm{P}-\mathrm{M},{ }^{\circ} \mathrm{F}$ & 440 & & 480 \\
\hline Solubility in trichloroethylene, $\%$ & 99 & & 99.9 \\
\hline \multicolumn{4}{|l|}{ Tests on residue from RTFC: } \\
\hline Absolute viscosity $0140^{\circ} \mathrm{F}$, ps & 2500 & 5000 & 3260 \\
\hline Kinematic viscosity @ $275^{\circ} \mathrm{F}$, cs & 275 & & 414.6 \\
\hline $\begin{array}{l}\text { Penetration } a 77^{\circ} \mathrm{F}, 100 \mathrm{~g}, 5 \mathrm{~s} \\
\quad \text { (tenths of } \mathrm{mm})\end{array}$ & 40 & & 53 \\
\hline Ductility o $45^{\circ} \mathrm{F},(1 \mathrm{~cm} / \mathrm{min}) \mathrm{cm}$ & 10 & & $50+$ \\
\hline
\end{tabular}

TABLE B.2. Hydraulic Asphalt Concrete Aggregate Specifications

\begin{tabular}{cc} 
Grid Size & \$Passing \\
\cline { 1 - 1 } $1 / 2$ & 100 \\
$3 / 8$ & 100 \\
4 & 77 \\
8 & 60 \\
10 & 54 \\
30 & 44 \\
50 & 23 \\
100 & 17 \\
200 & 15
\end{tabular}

B. 1 
TABLE B.3. Specifications of Catalytic Airblown Asphalt

Catalytic Airblown Asphalt

ASTM No. D2521

\begin{tabular}{|c|}
\hline Property \\
\hline $\begin{array}{l}\text { Softening Point }\left({ }^{\circ} \mathrm{C}\right) \\
\left(175^{\circ} \mathrm{F}\right)\end{array}$ \\
\hline Penetration $825^{\circ} \mathrm{C}\left(77^{\circ} \mathrm{F}\right), 100 \mathrm{~g}, 5 \mathrm{~s}$ \\
\hline $\begin{array}{l}\text { Penetration }{ }^{\circ} 0^{\circ} \mathrm{C}\left(32^{\circ} \mathrm{F}\right), 200 \mathrm{~g}, 60 \mathrm{~s} \\
\quad(\text { tenths of } \mathrm{mm})\end{array}$ \\
\hline $\begin{array}{l}\text { Penetration } 046^{\circ} \mathrm{C}\left(115^{\circ} \mathrm{F}\right), 50 \mathrm{~g}, 5 \mathrm{~s} \\
\quad \text { (tenths of mm) }\end{array}$ \\
\hline Flash Point $\left({ }^{\circ} \mathrm{C}\right)$ \\
\hline Solubility in Trichloroethylene (wt\%) \\
\hline Ductility $025^{\circ} \mathrm{C}\left(77^{\circ} \mathrm{F}\right), 0.083 \mathrm{~cm} / \mathrm{s}(\mathrm{cm})$ \\
\hline Specific Gravity $225^{\circ} \mathrm{C}\left(77^{\circ} \mathrm{F}\right)$ \\
\hline Specific Gravity $015.6^{\circ} \mathrm{C}\left(60^{\circ} \mathrm{F}\right)$ \\
\hline $\begin{array}{l}\text { Penetration } 225^{\circ} \mathrm{C}\left(77^{\circ} \mathrm{F}\right), 100 \mathrm{~g}, 5 \mathrm{~s} \\
\text { after heating to } 163^{\circ} \mathrm{C} \text { for } 5 \text { hours } \\
(\% \text { of origina } 1)\end{array}$ \\
\hline Loss $@ 163^{\circ} \mathrm{C}, 5$ hours (wt\%) \\
\hline
\end{tabular}

\begin{tabular}{|c|c|c|}
\hline Minimum & Maximum & $\begin{array}{l}n \\
\text { Typical } \\
\text { Analysis }\end{array}$ \\
\hline $\begin{array}{c}79 \\
\left(175^{\circ} \mathrm{F}\right)\end{array}$ & 93 & 88 \\
\hline 50 & 60 & 56 \\
\hline \multirow[t]{2}{*}{30} & & 32 \\
\hline & 120 & 114 \\
\hline 218 & & 232 \\
\hline 97.0 & & 99.0 \\
\hline \multirow[t]{3}{*}{3.5} & & 6.0 \\
\hline & & 1.027 \\
\hline & & 1.031 \\
\hline \multirow[t]{2}{*}{60} & & 72 \\
\hline & 1.0 & 0.1 \\
\hline
\end{tabular}

B. 2 
APPENDIX C

EXPOSURE CONDITIONS, PERMEABILITIES,

AND REACTION RATES 


\section{APPENDIX C \\ EXPOSURE CONDITIONS, PERMEABILITIES, \\ AND REACTION RATES}

This appendix presents the exposure conditions, permeabilities, and integrated infrared absorption band data for the aged liners. Reference Codes $A, B$, and $D$ through $G$ refer to the first set of exposure tests. Codes 1 through 7 refer to the second set of exposure tests; and Code $Z$ is the control, unexposed asphalt. 

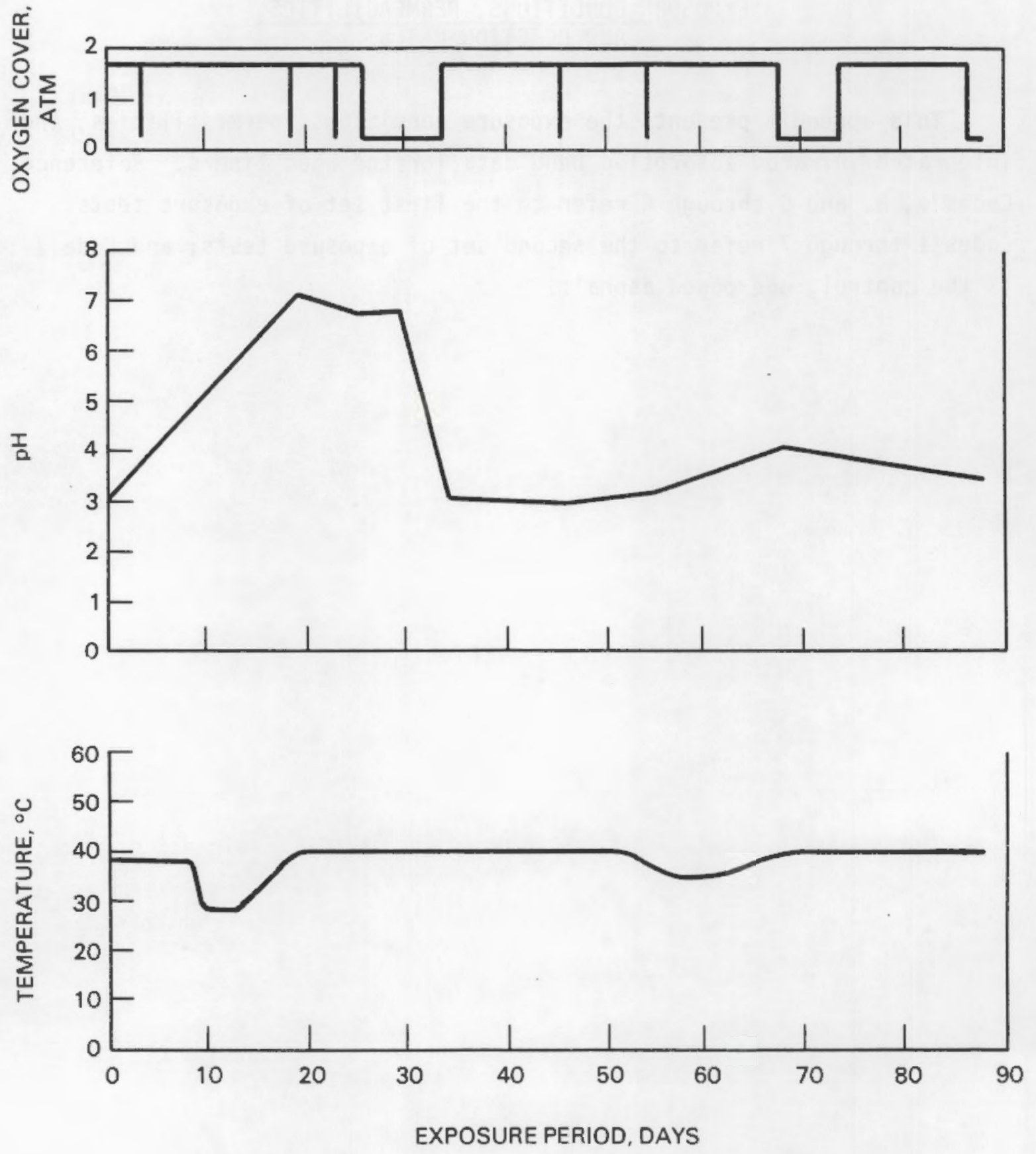

FIGURE C.1. Exposure Conditions for Liner A 

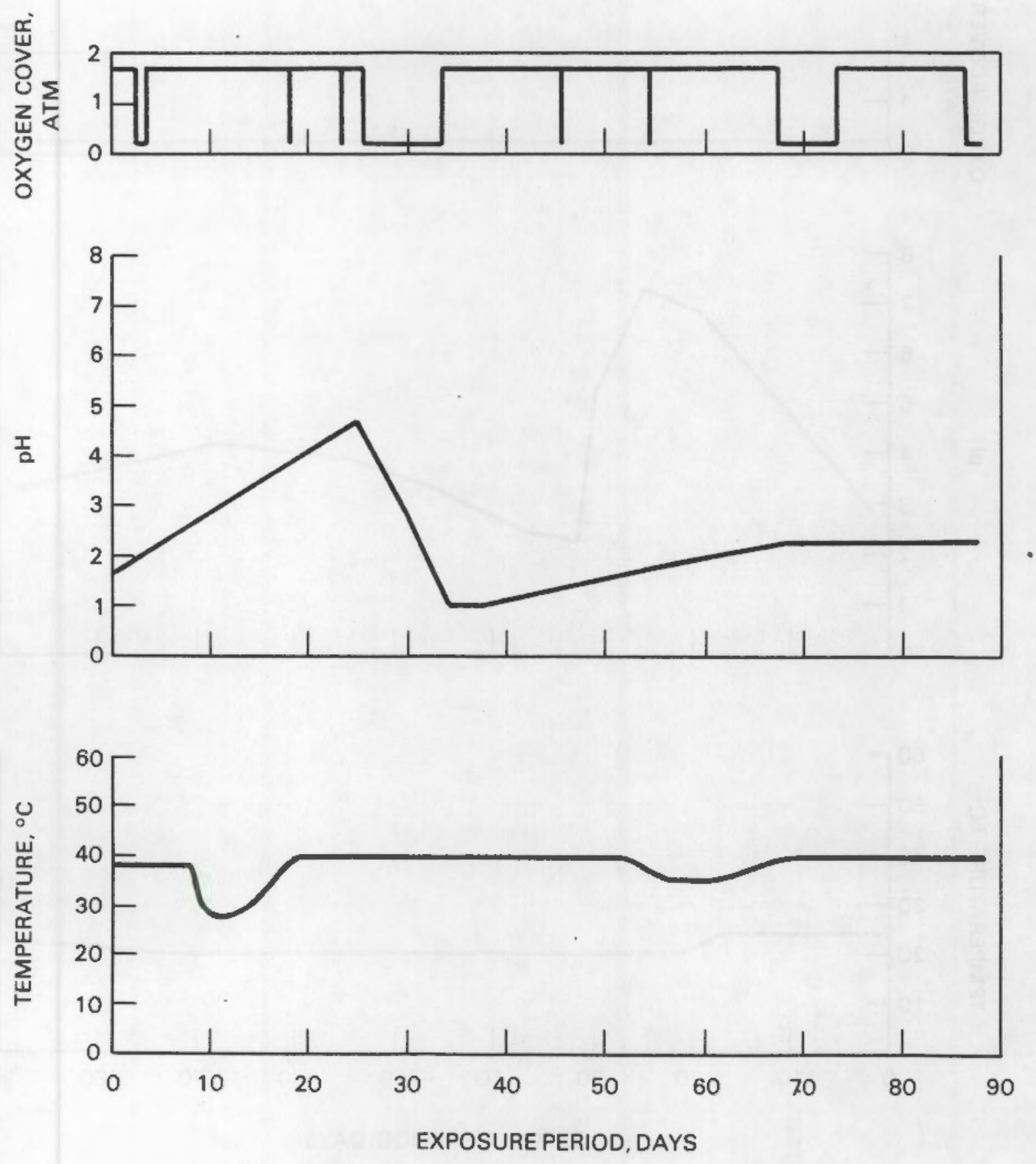

FIGURE C.2. Exposure Conditions for Liner B 

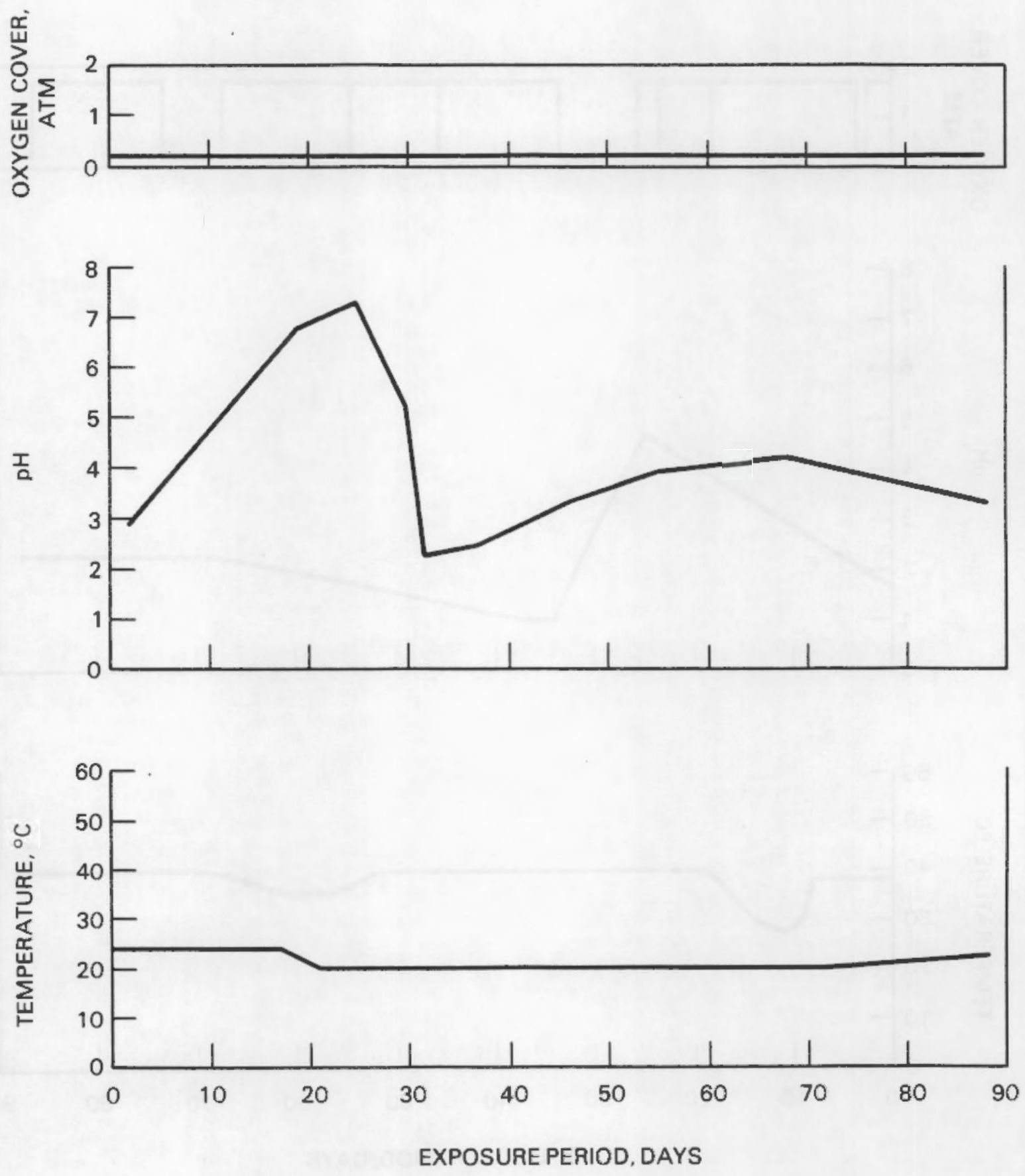

FIGURE C.3. Exposure Conditions for Liner D 

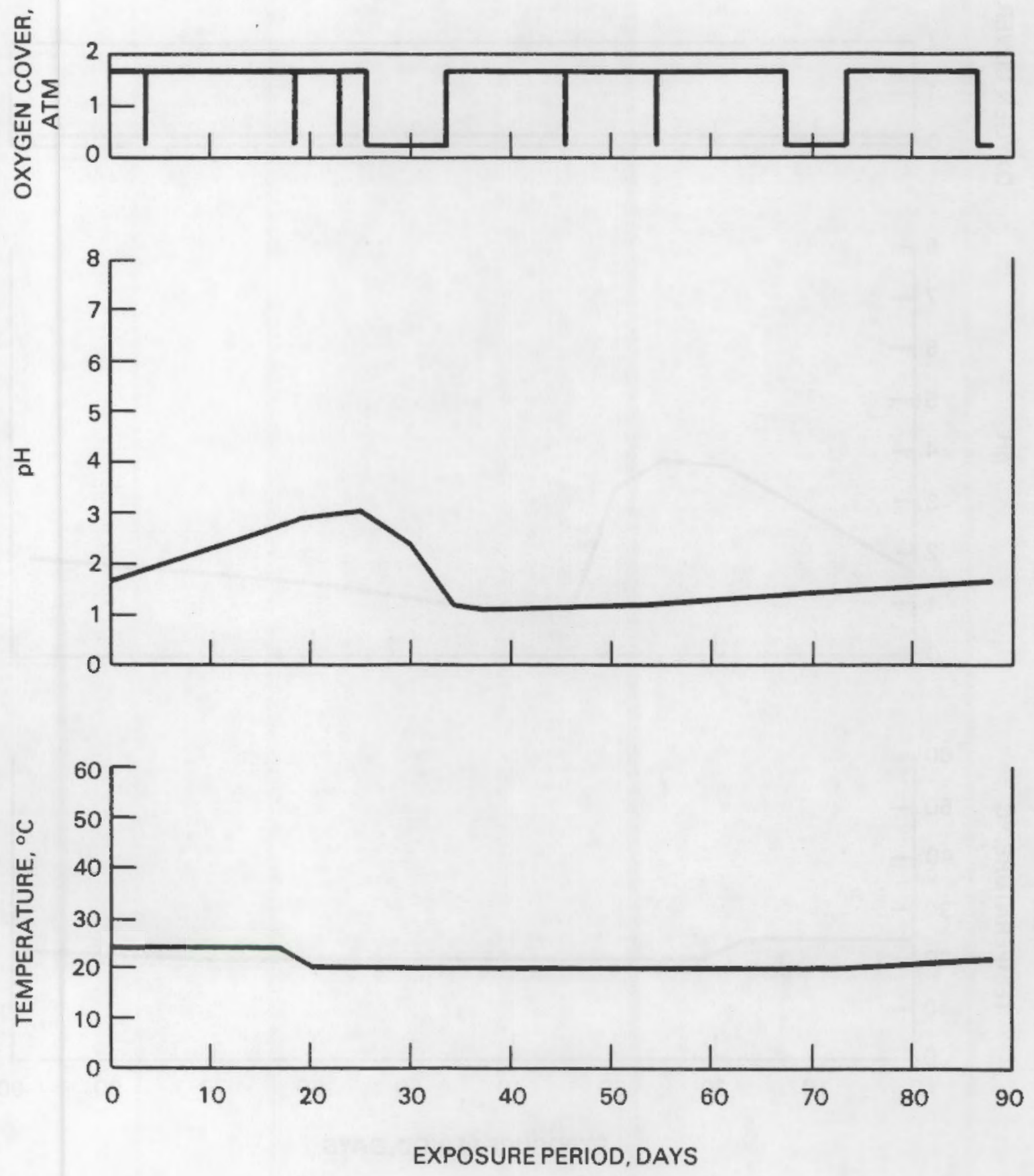

FIGURE C.4. Exposure Conditions for Liner E

C.5 

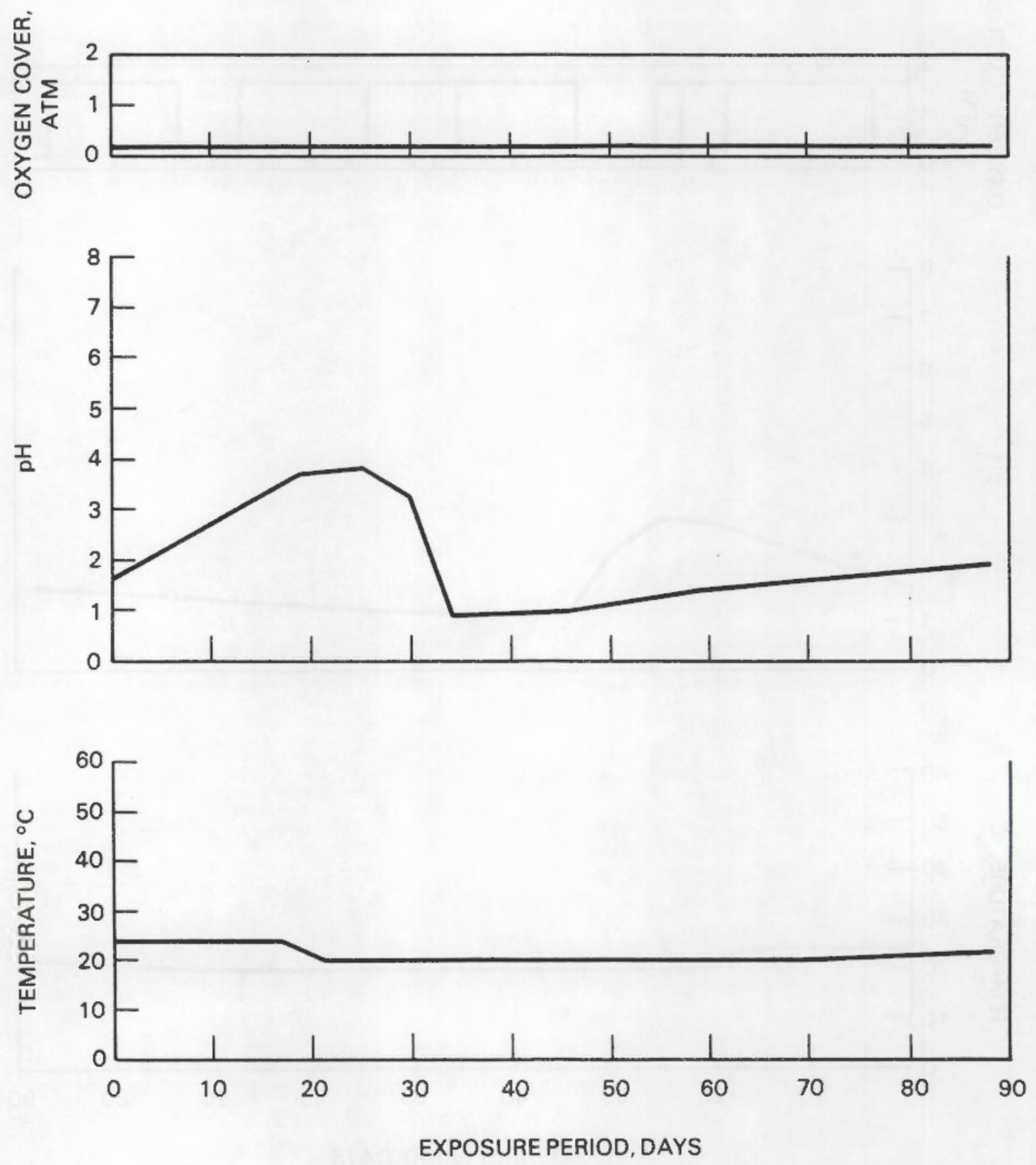

FIGURE C.5. Exposure Conditions for Liner F

$$
\text { C.6 }
$$



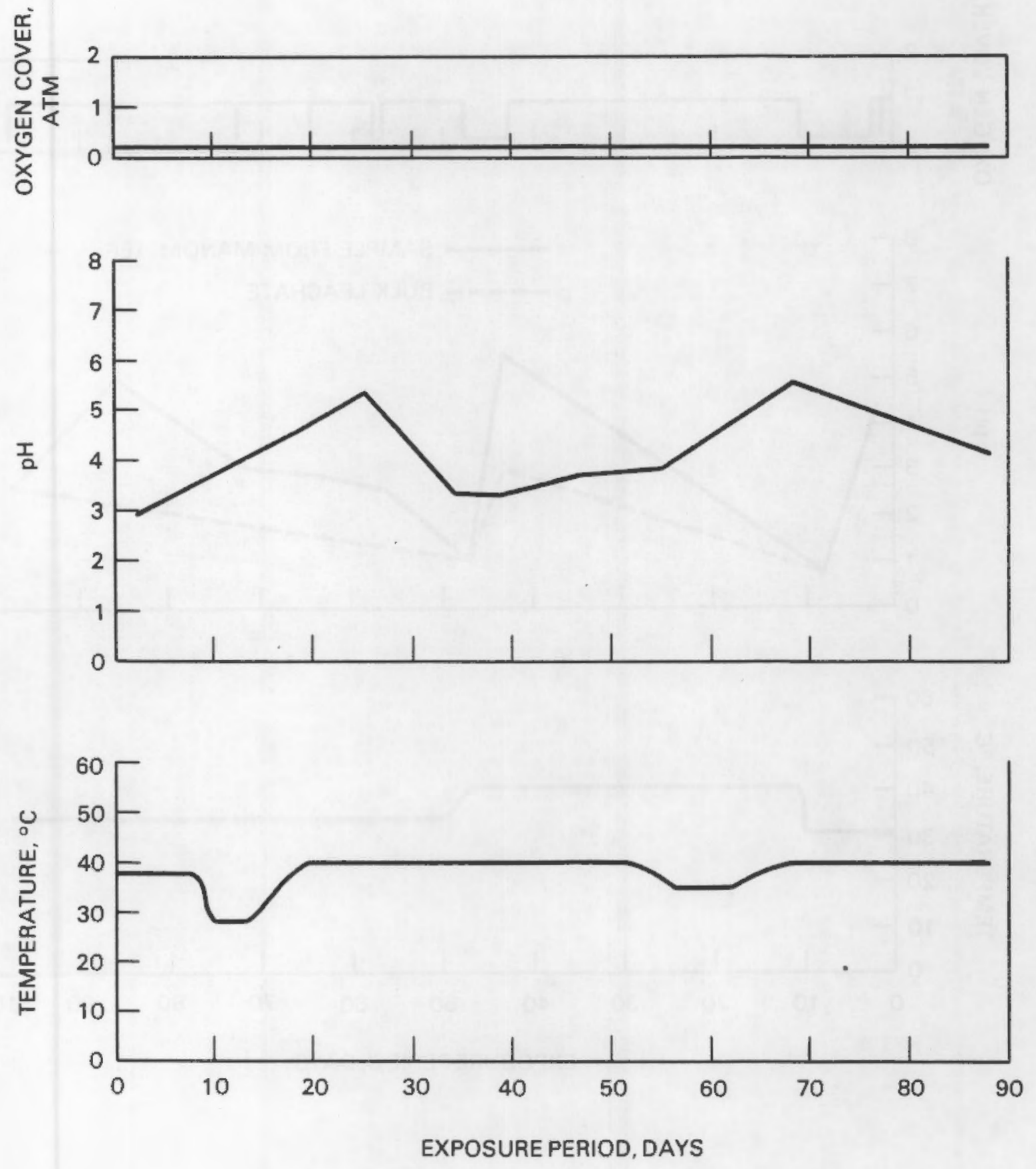

FIGURE C.6. Exposure Conditions for Liner G 

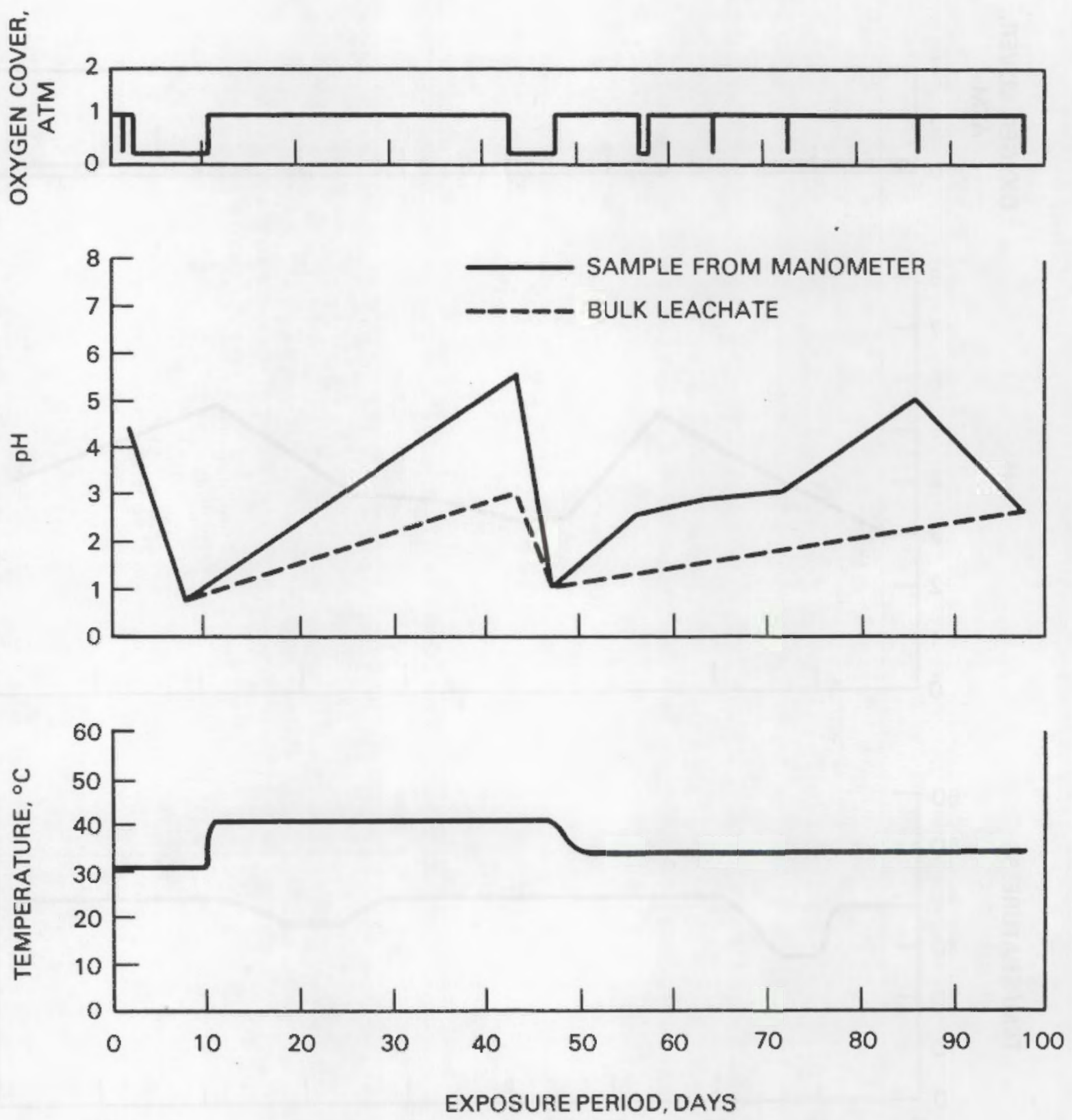

FIGURE C.7. Exposure Conditions for Liner 1 

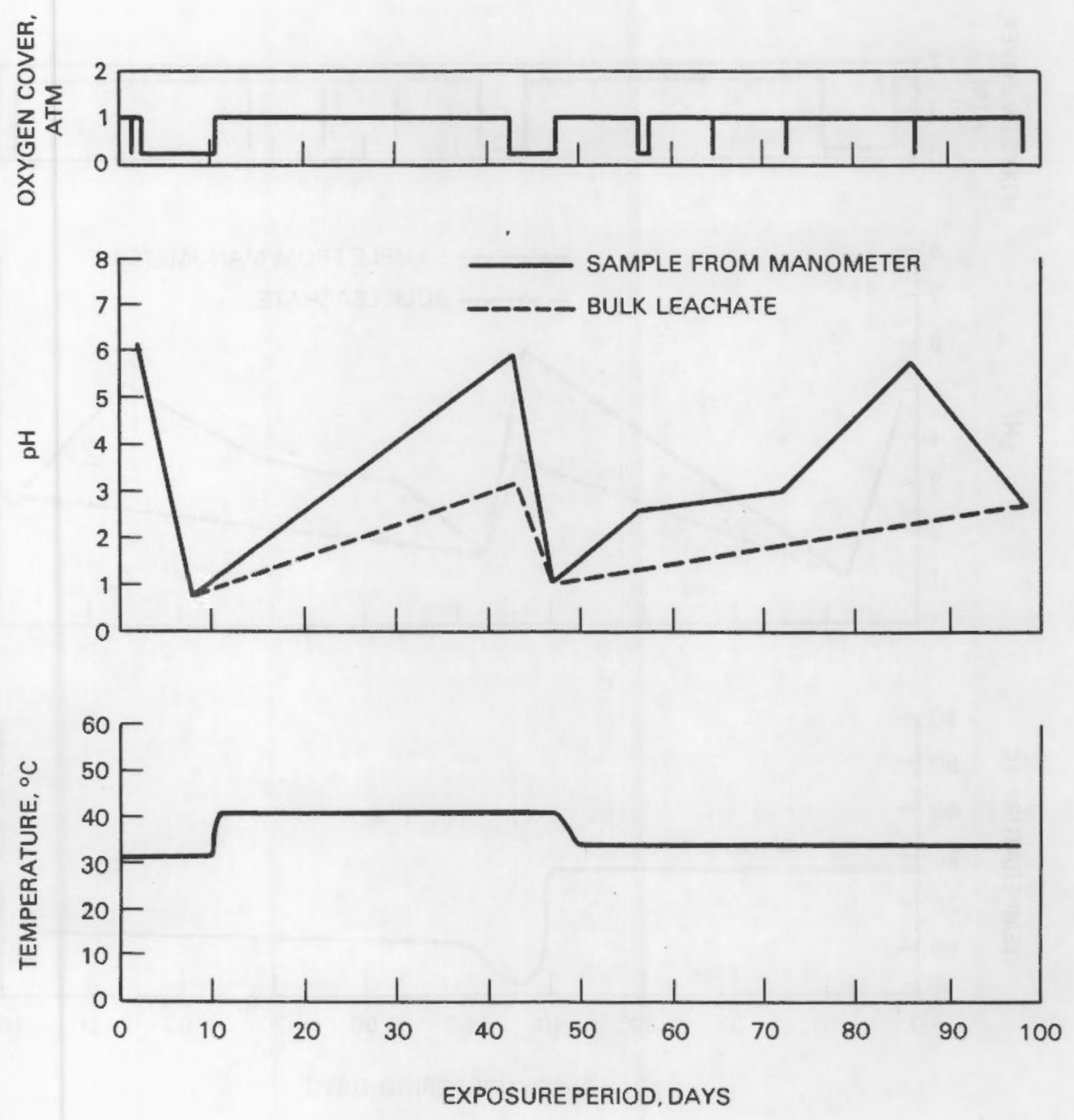

FIGURE C.8. Exposure Conditions for Liner 2 

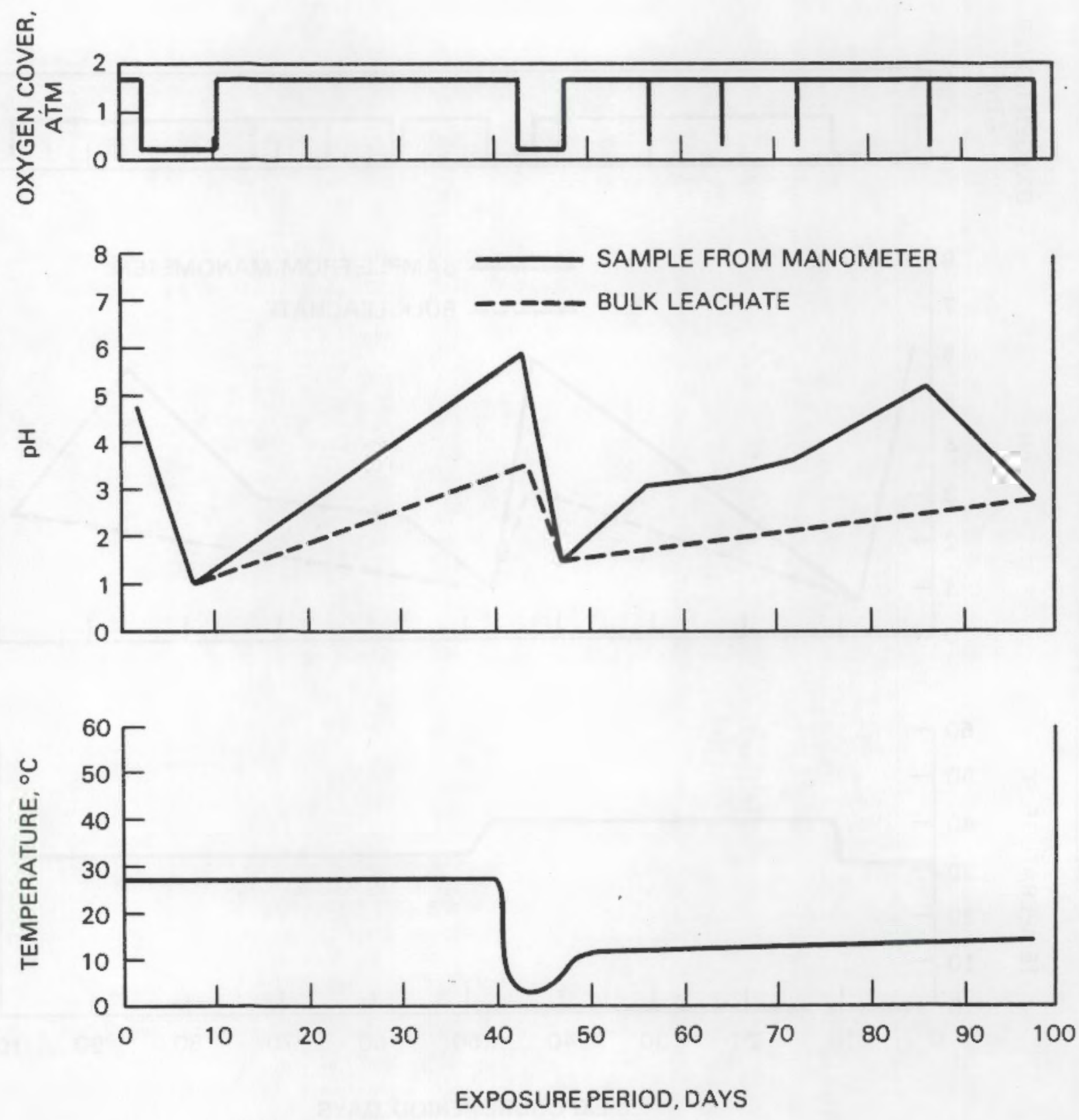

FIGURE C.9. Exposure Conditions for Liner 3 

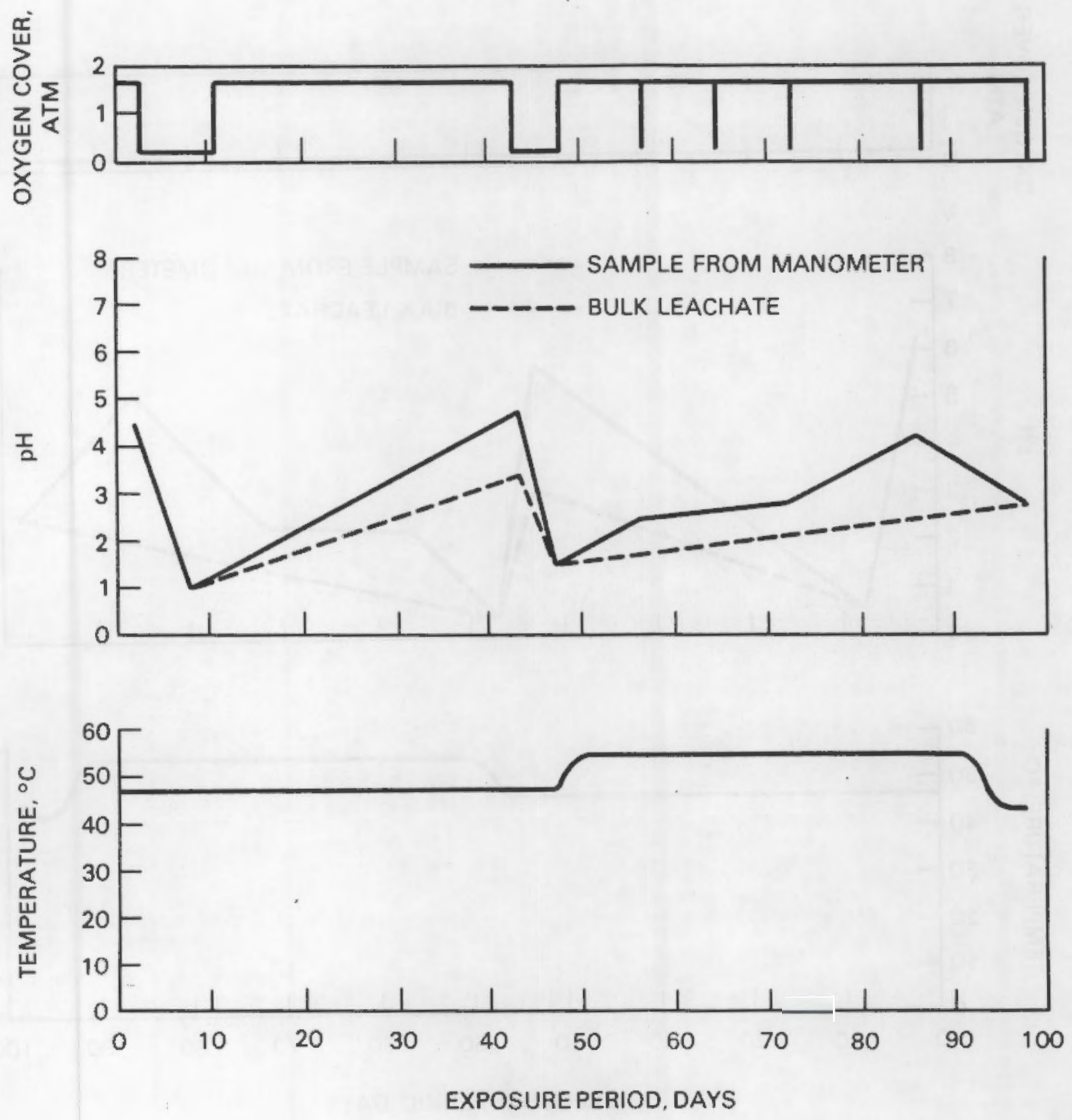

FIGURE C.10. Exposure Conditions for Liner 4 

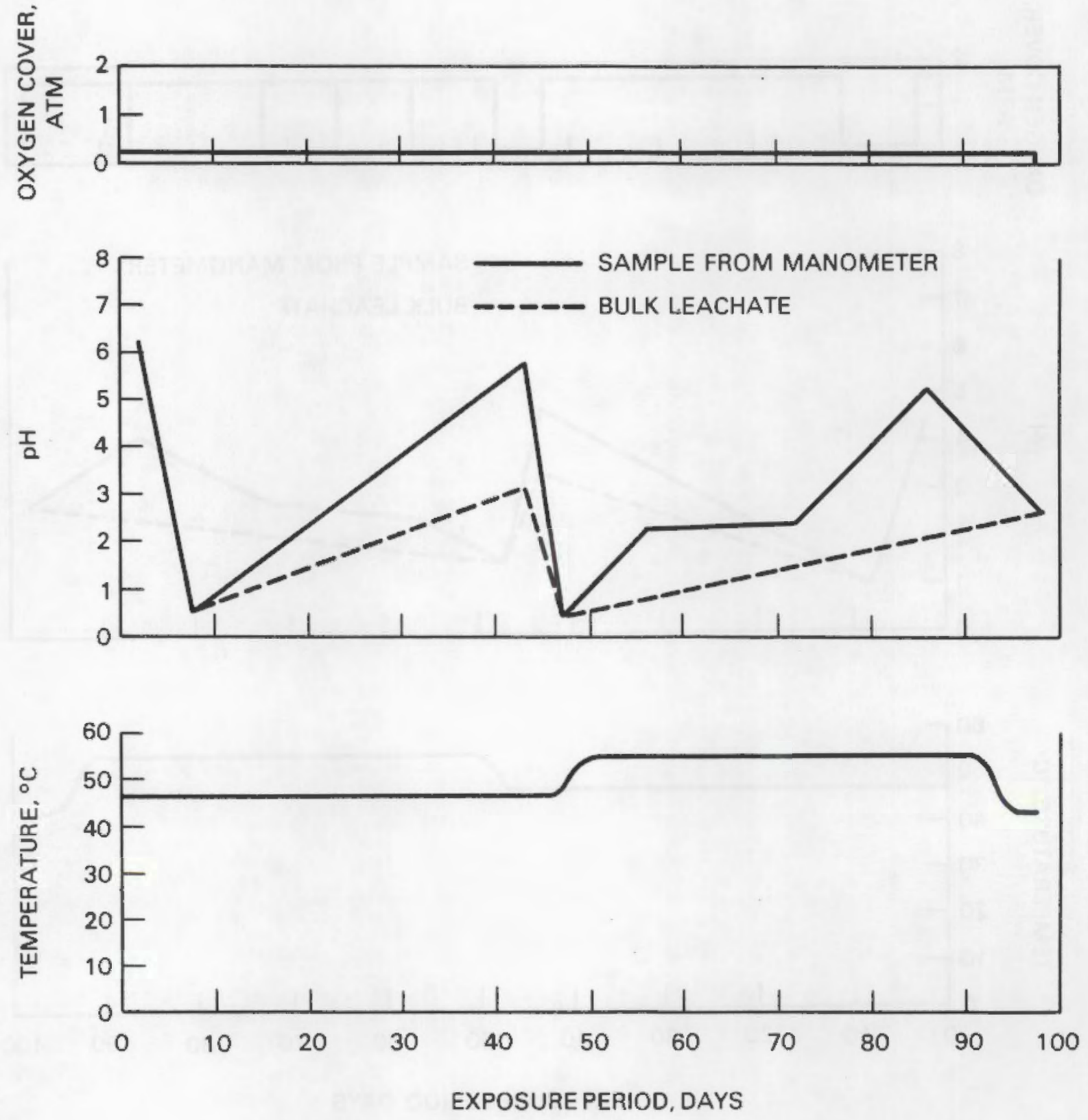

FIGURE C.11. Exposure Conditions for Liner 5 

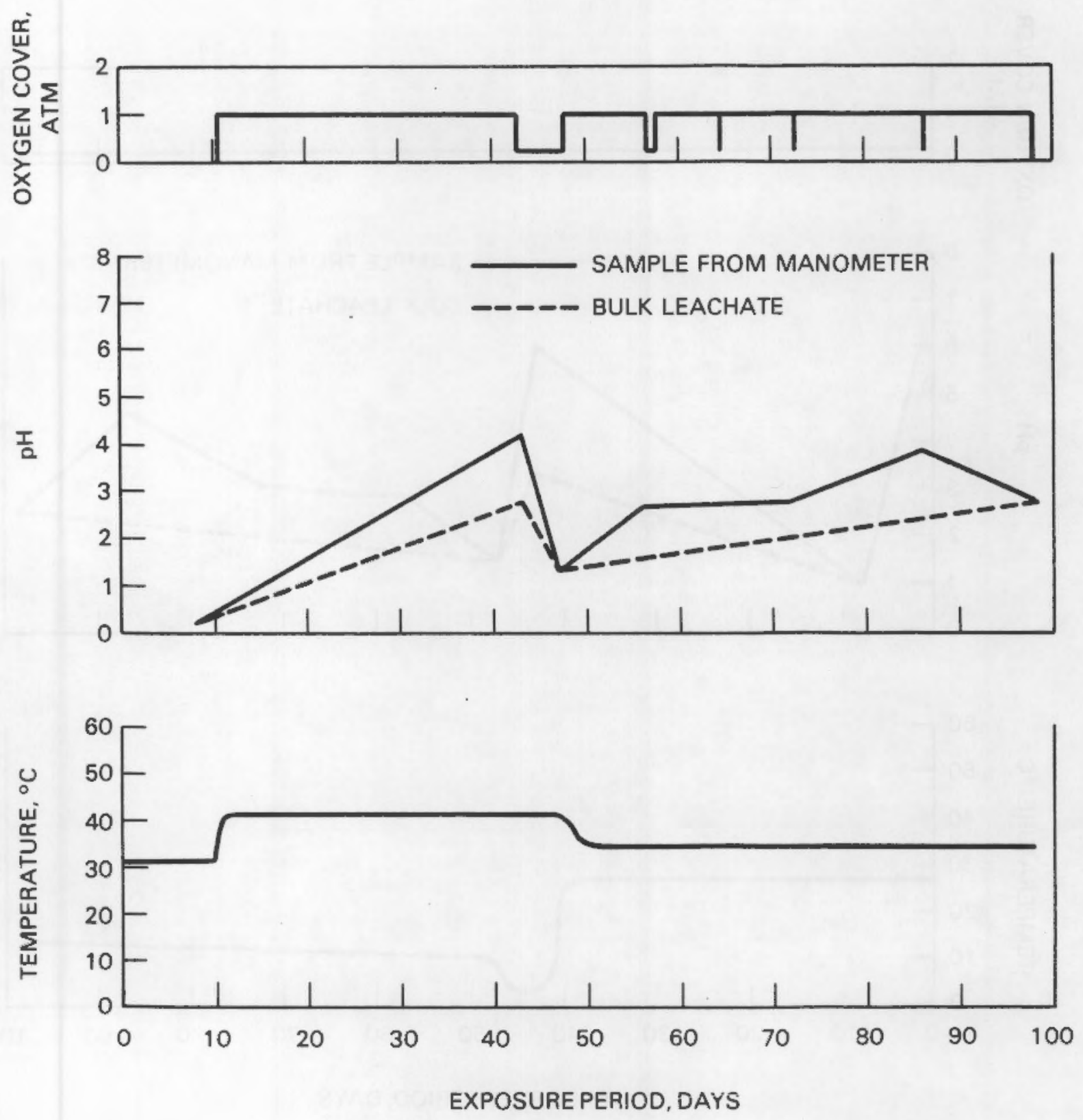

FIGURE C.12. Exposure Conditions for Liner 6 

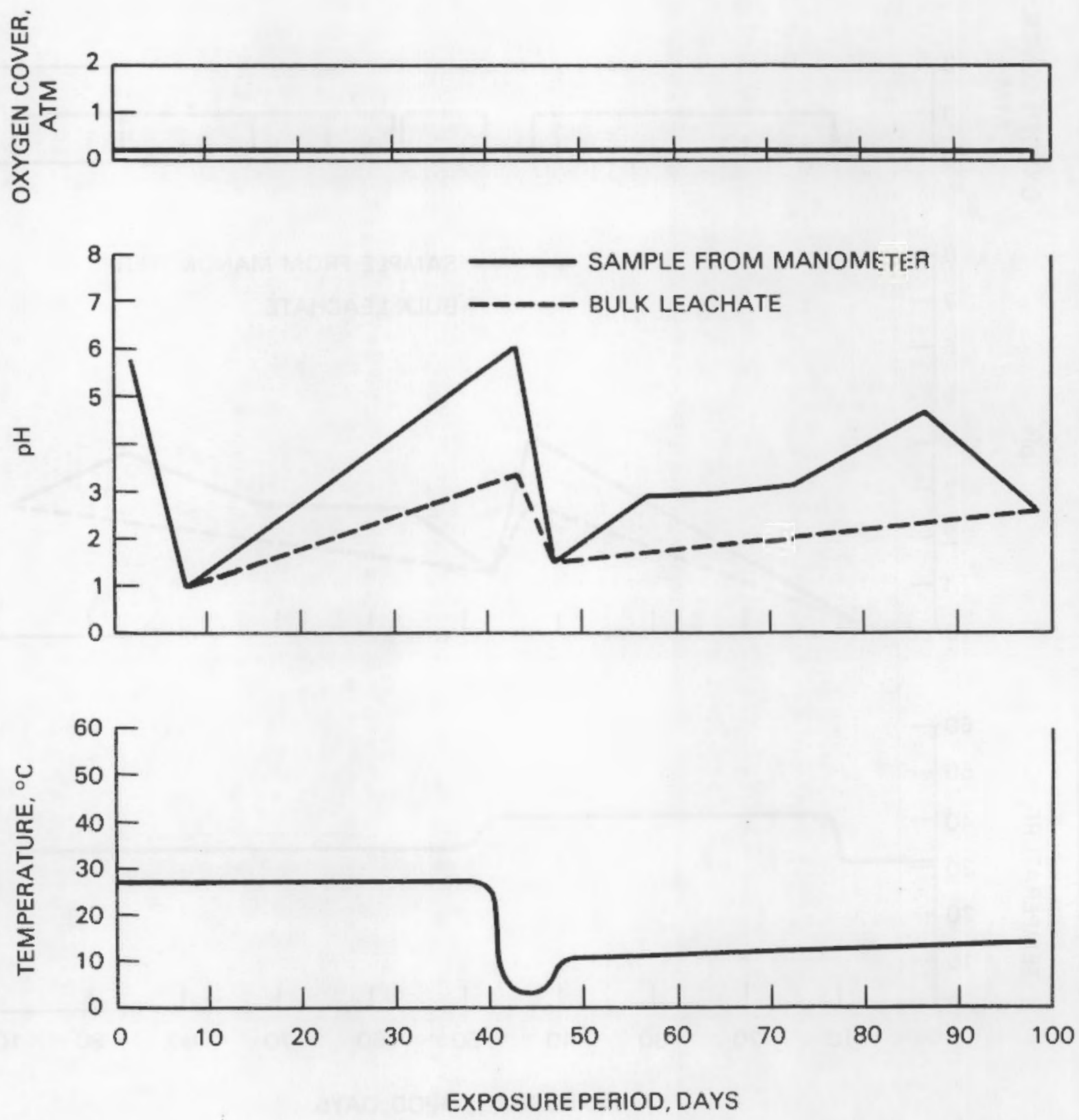

FIGURE C.13. Exposure Conditions for Liner 7 


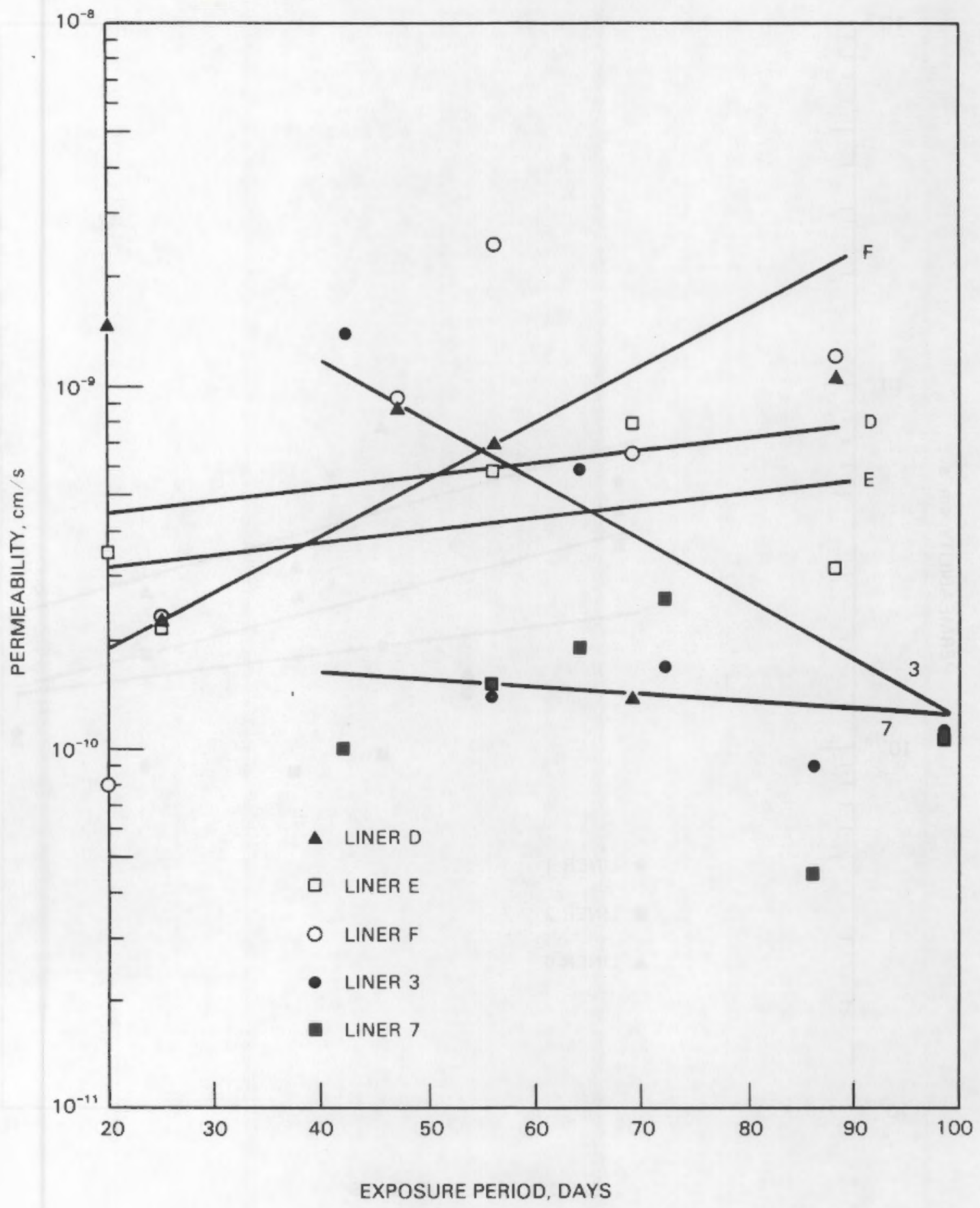

FIGURE C.14. Permeabilities of Liners Exposed To Low Temperature Conditions 


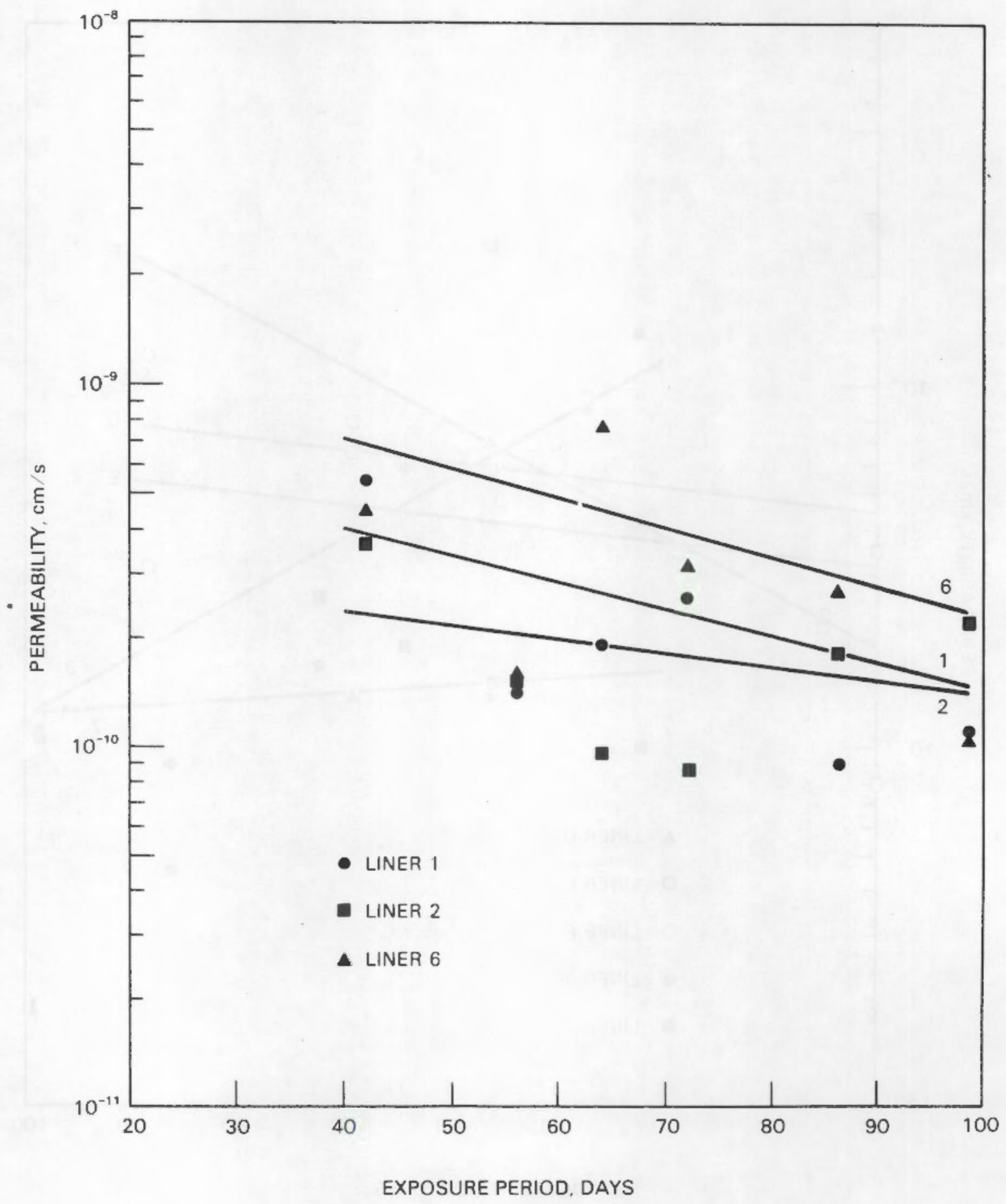

FIGURE C.15. Permeabilities of Liners Exposed to Intermediate Temperature Conditions 


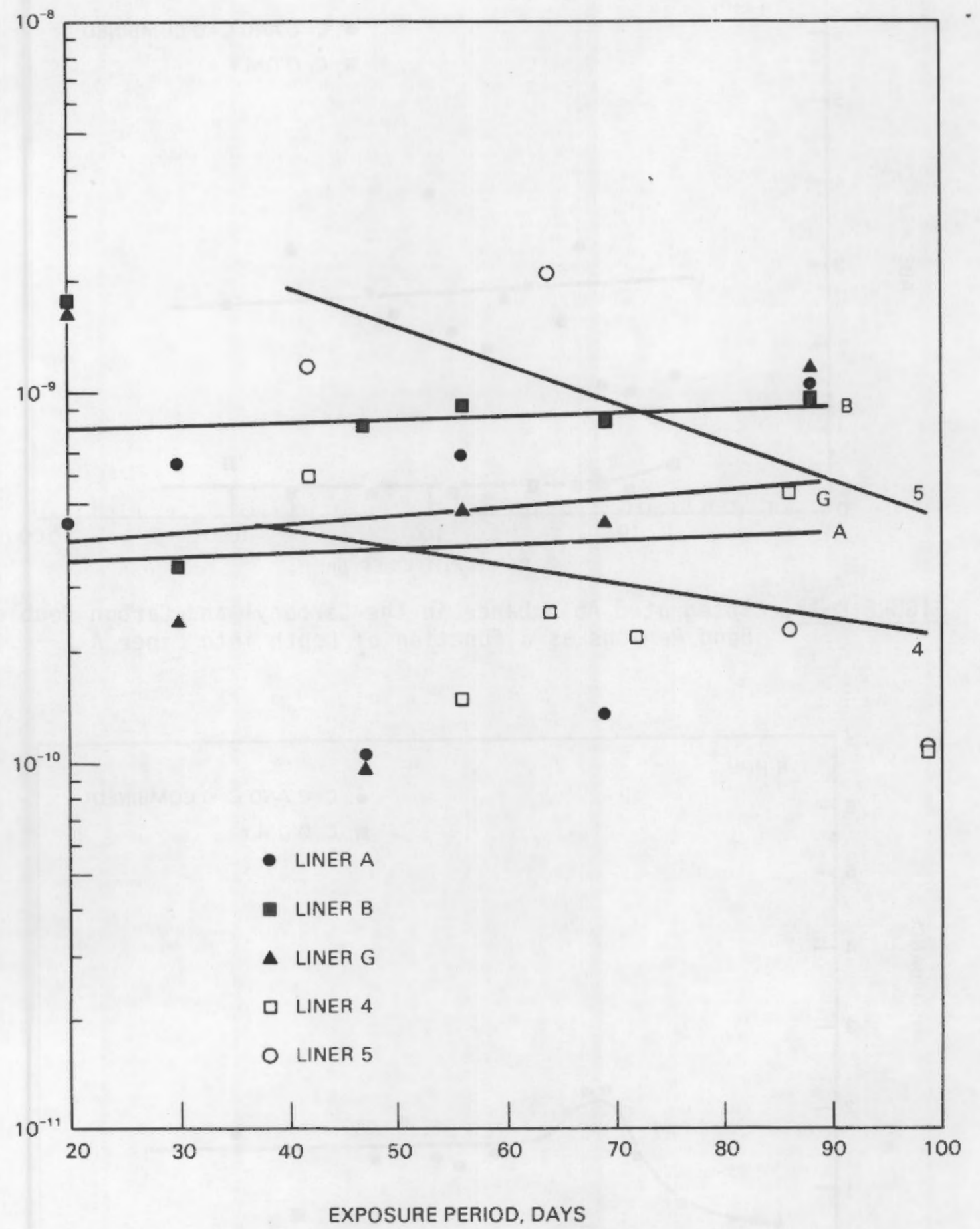

FIGURE C.16. Permeabilities of Liners Exposed to High Temperature Conditions 


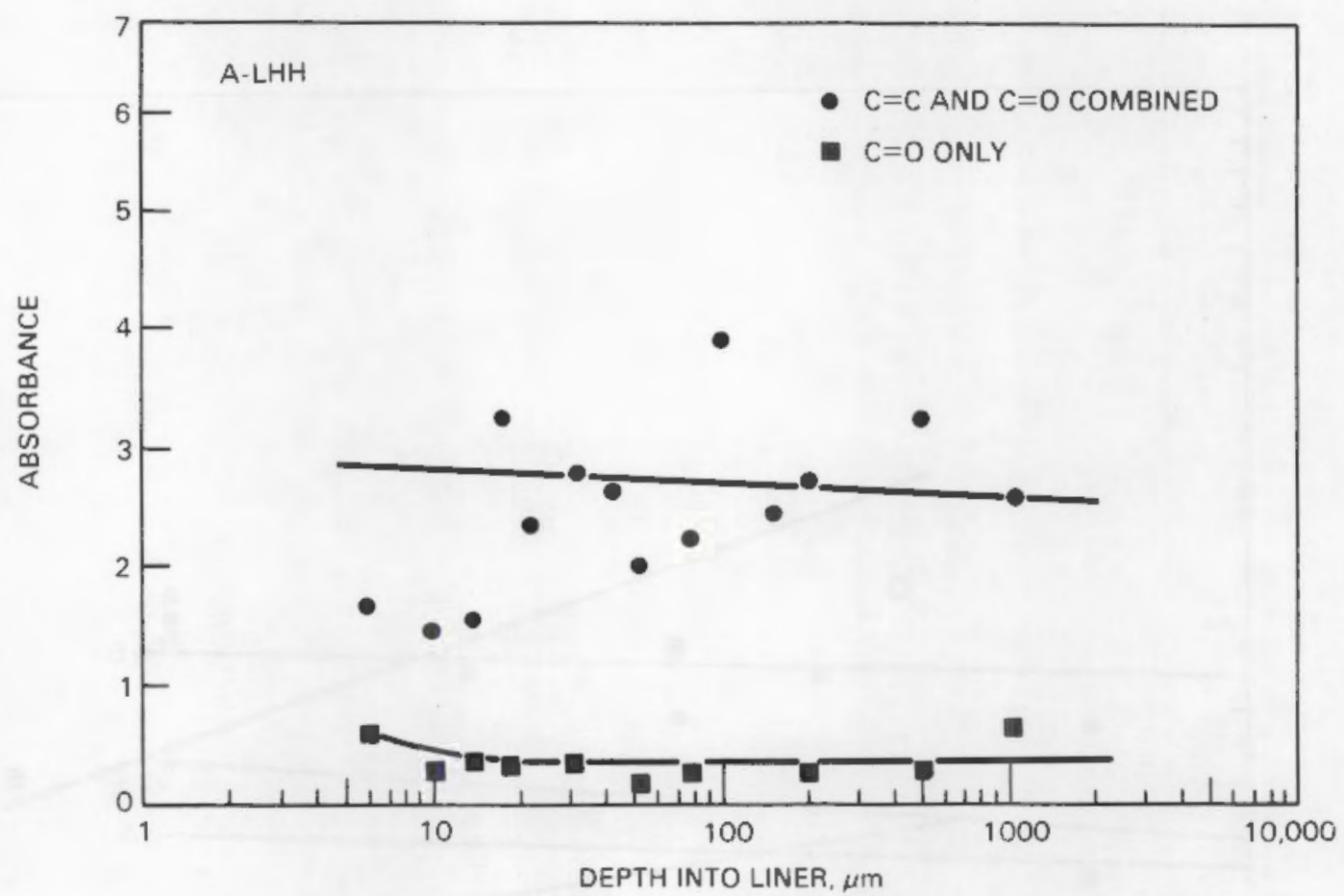

FIGURE C.17. Integrated Absorbance in the Carbonyl and Carbon Double Bond Regions as a Function of Depth into Liner A

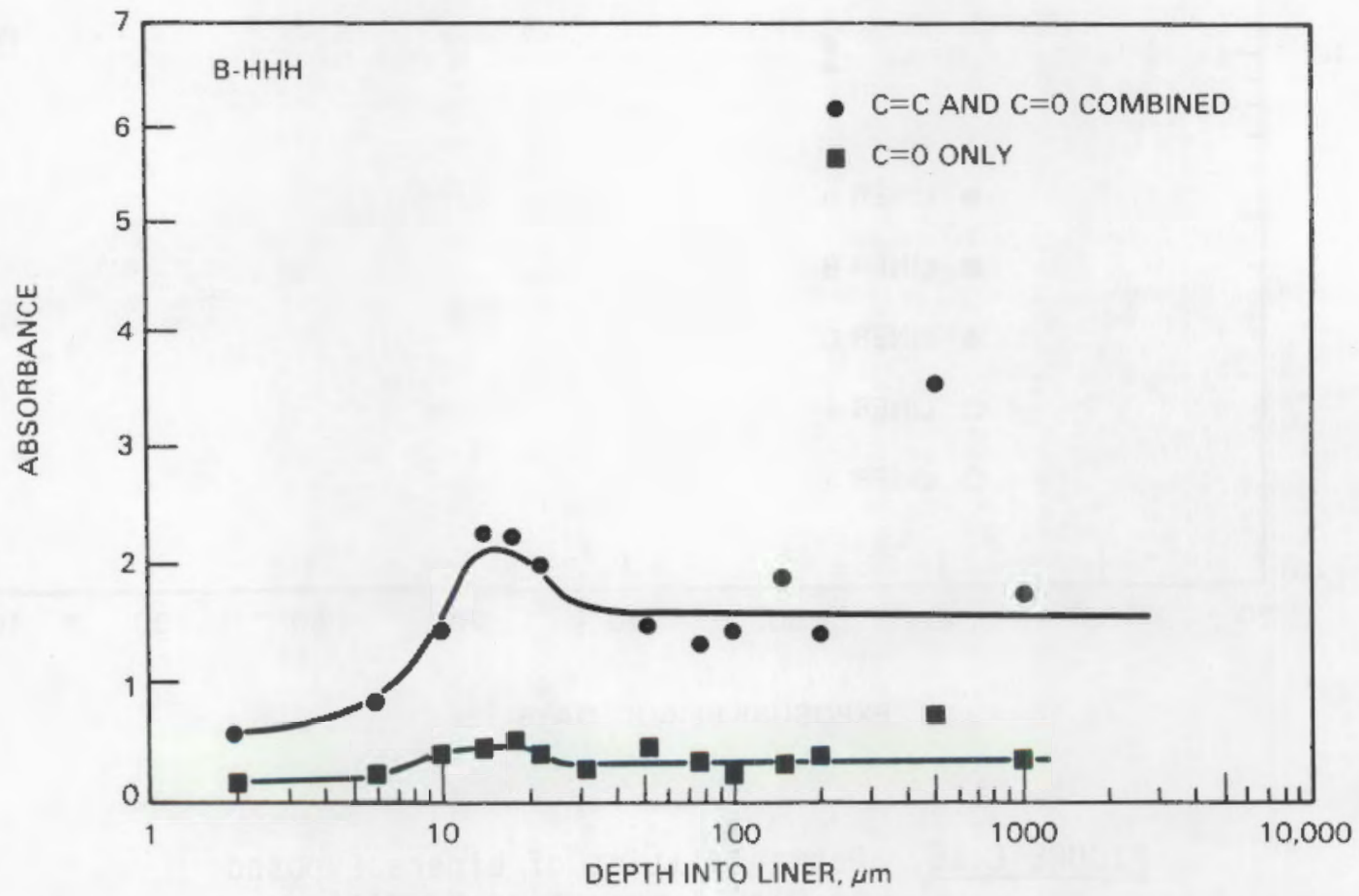

FIGURE C.18. Integrated Absorbance in the Carbonyl and Carbon Double Bond Regions as a Function of Depth into Liner B 


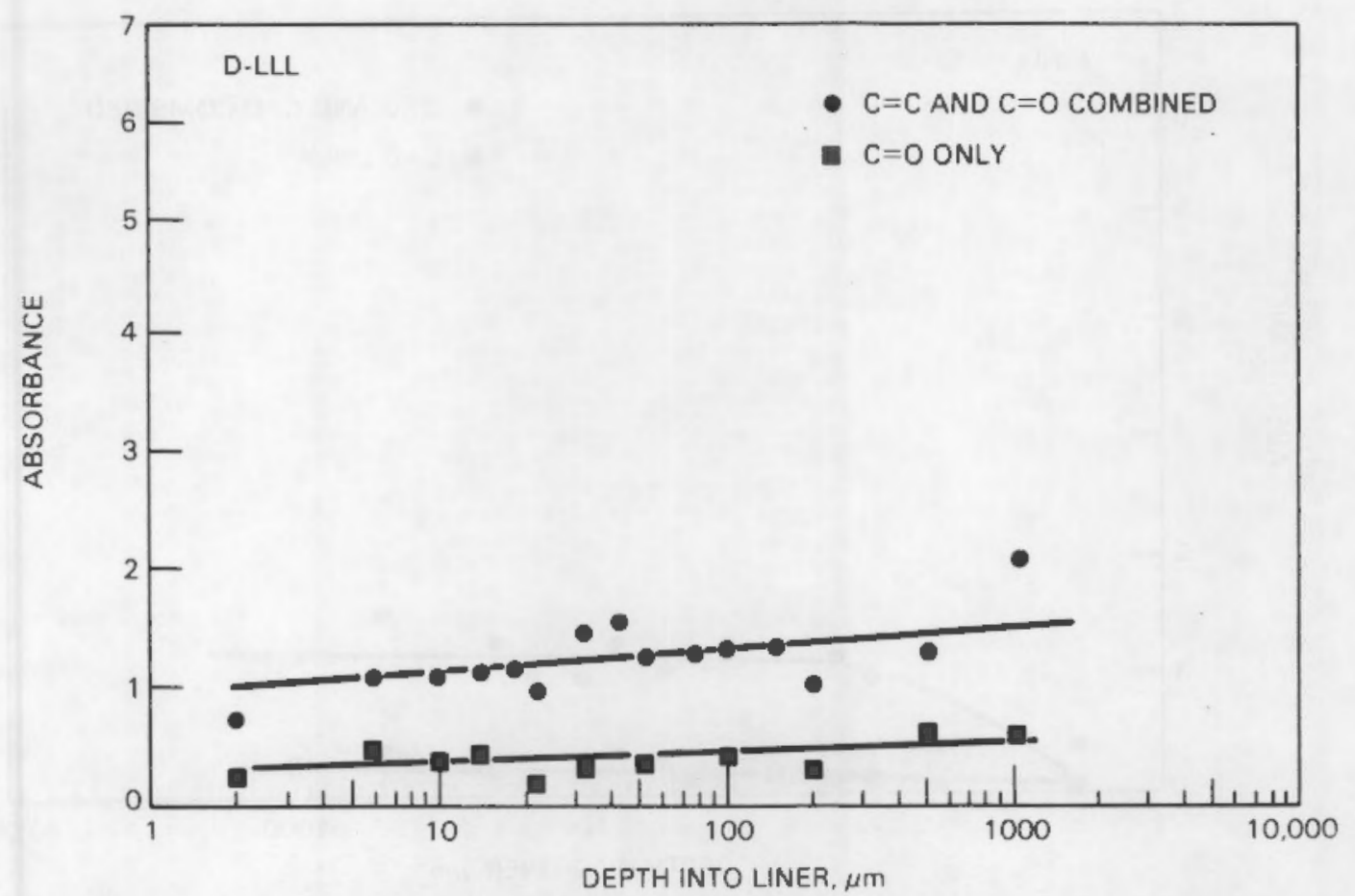

FIGURE C.19. Integrated Absorbance in the Carbonyl and Carbon Double Bond Regions as a Function of Depth into Liner D

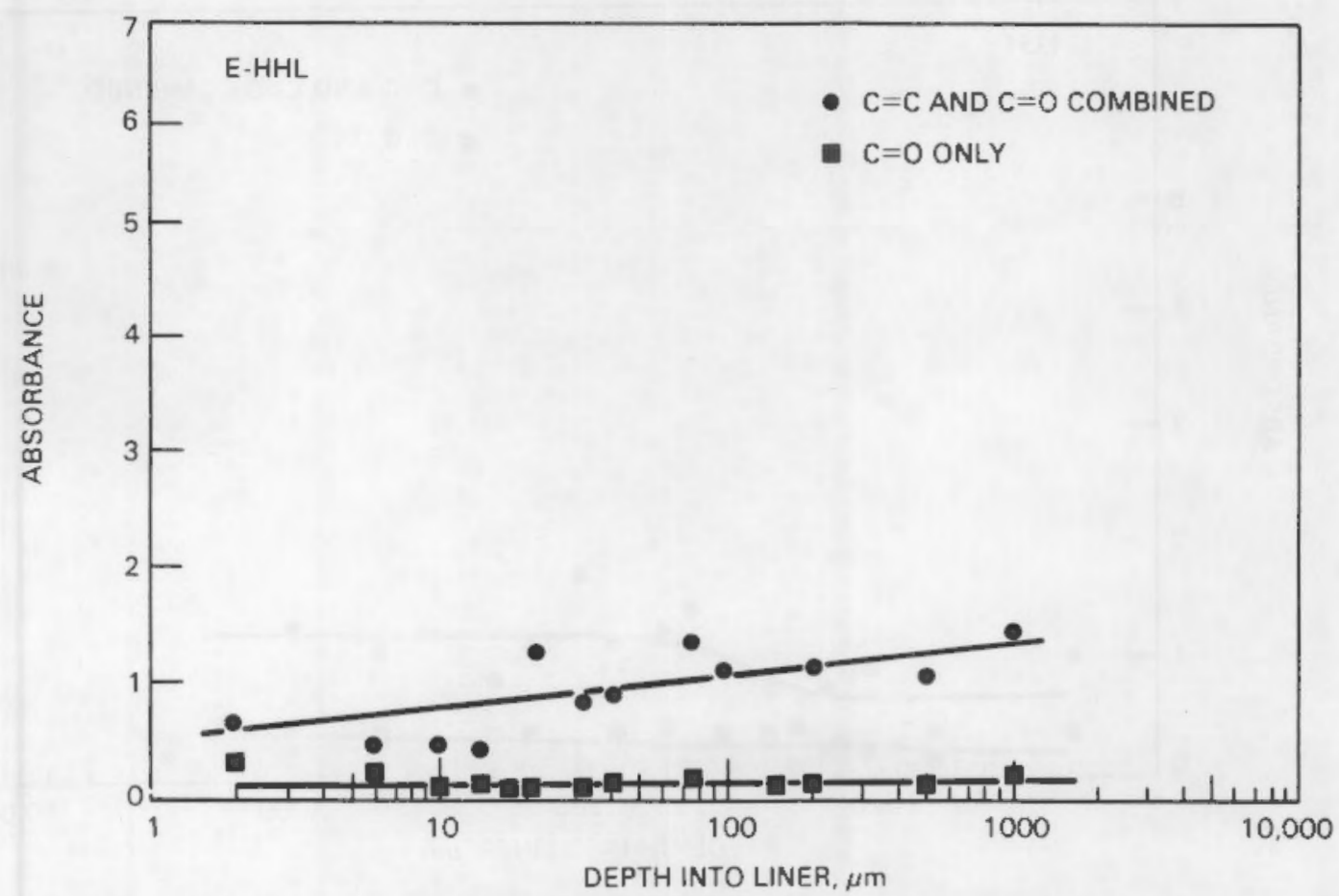

FIGURE C.20. Integrated Absorbance in the Carbonyl and Carbon Double Bond Regions as a Function of Depth into Liner $E$ 


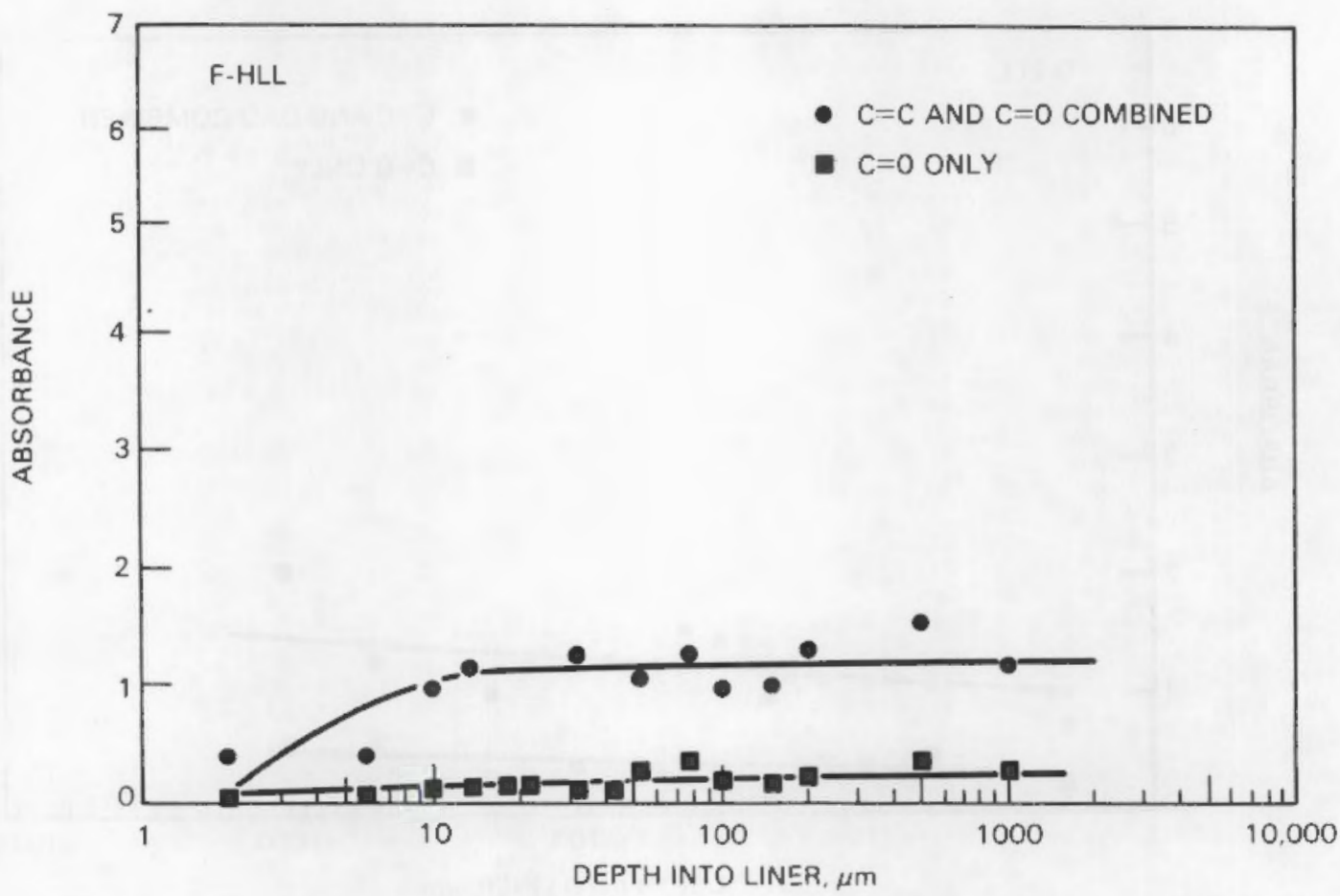

FIGURE C.21. Integrated Absorbance in the Carbonyl and Carbon Double Bond Regions as a Function of Depth into Liner F

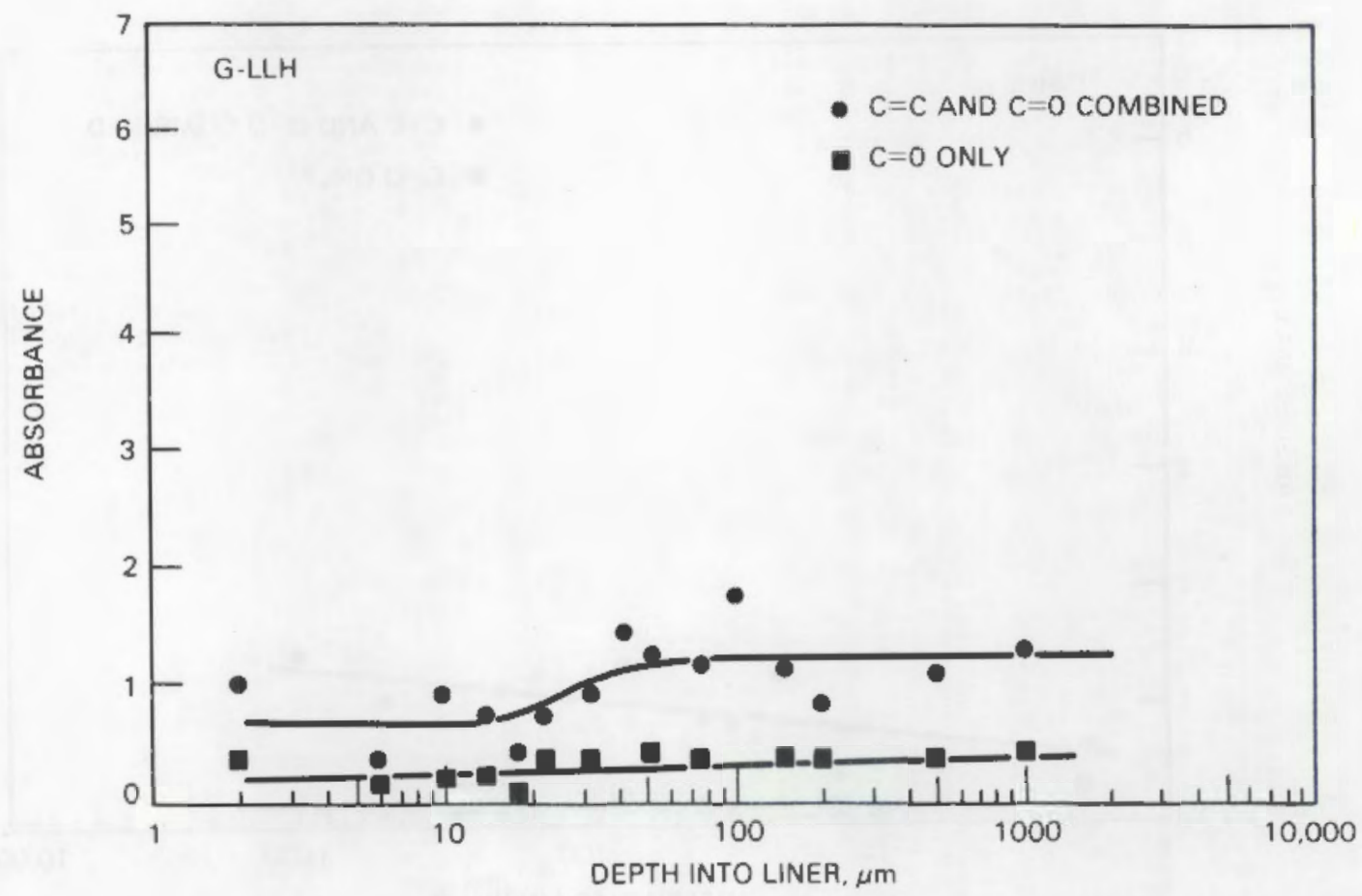

FIGURE C.22. Integrated Absorbance in the Carbonyl and Carbon Double Bond Regions as a Function of Depth into Liner G 


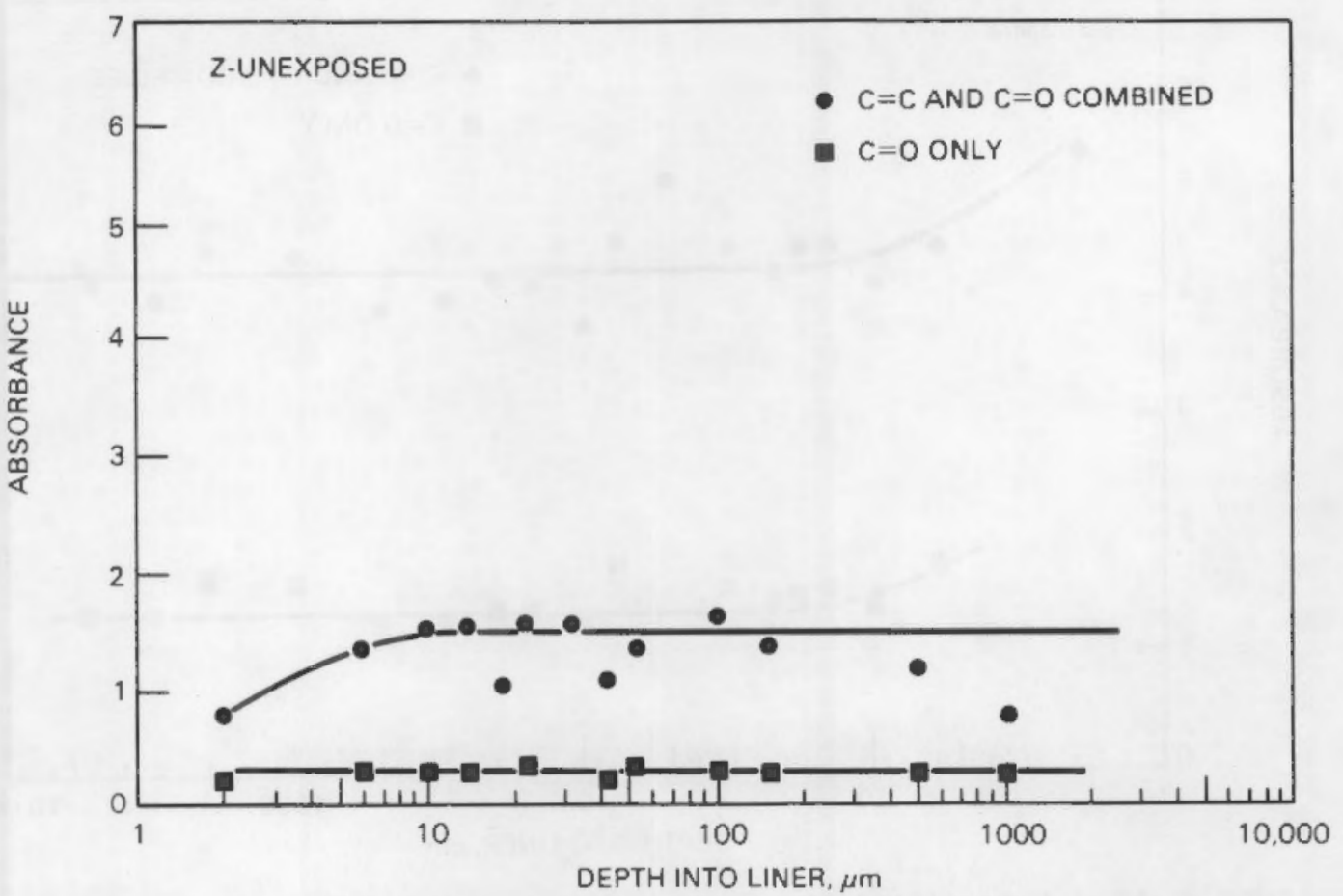

FIGURE C.23. Integrated Absorbance in the Carbonyl and Carbon Double Bond Regions as a Function of Depth into the Control Liner Z

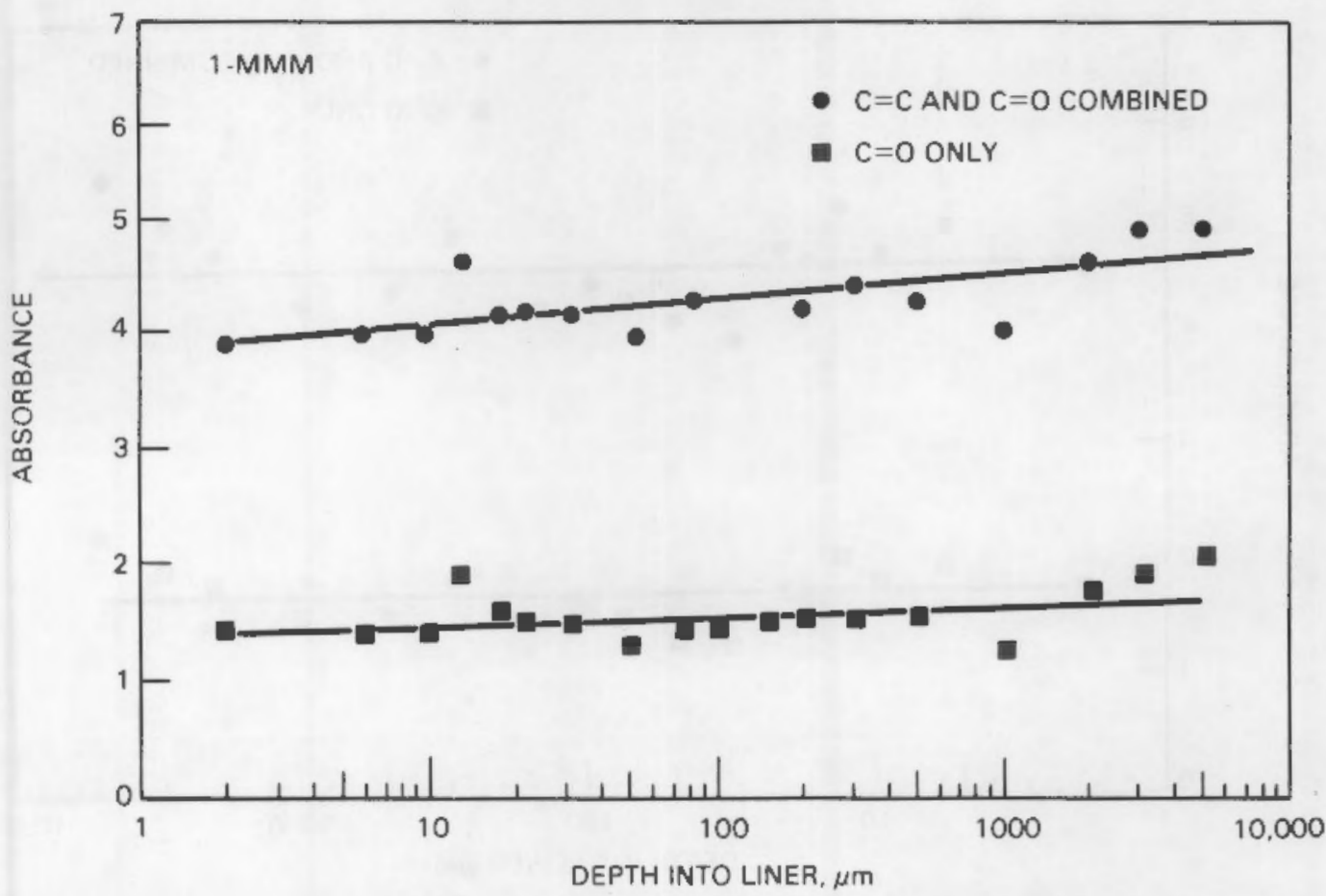

FIGURE C.24. Integrated Absorbance in the Carbonyl and Carbon Double Bond Regions as a Function of Depth into Liner 1 


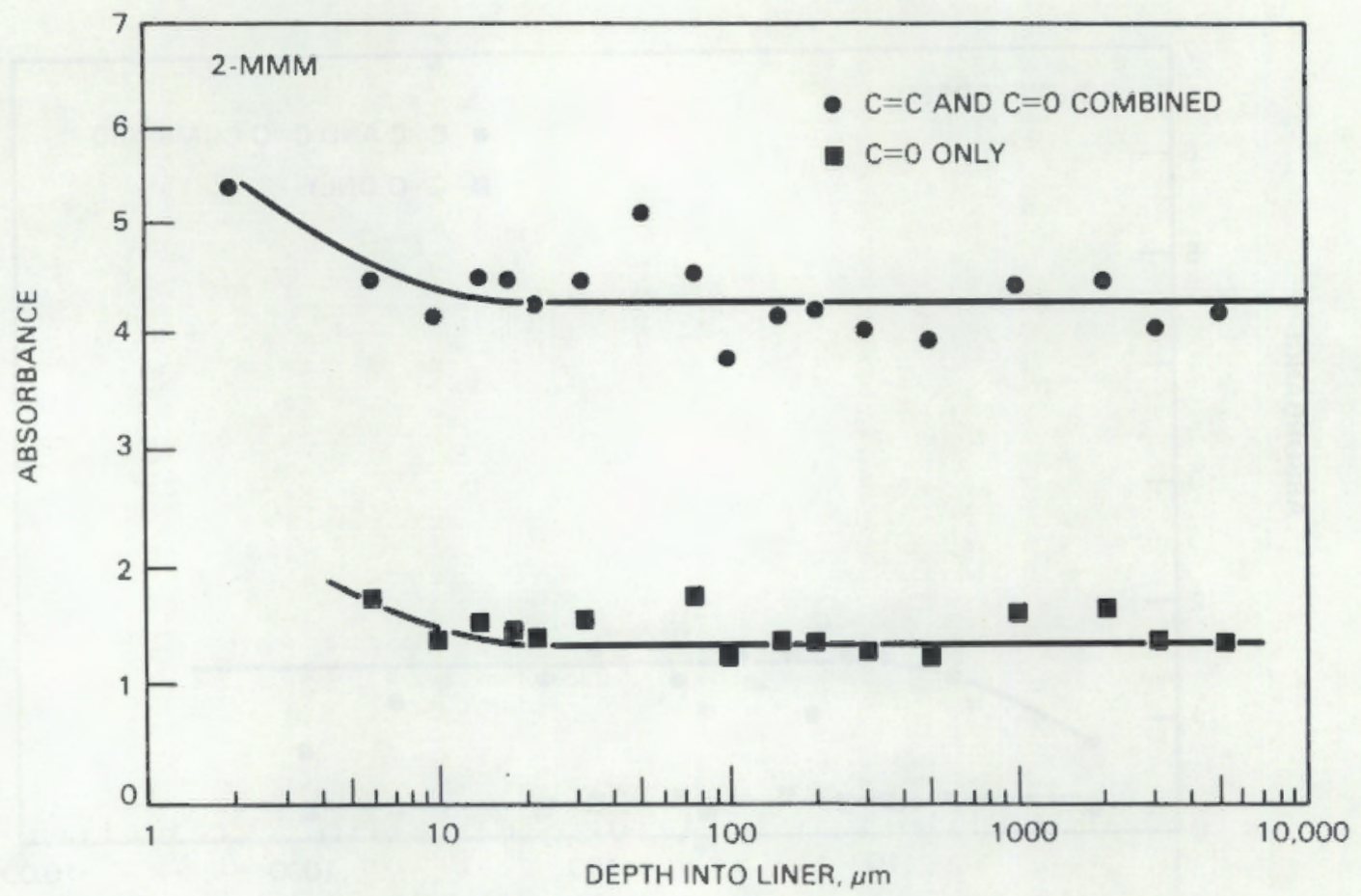

FIGURE C.25. Integrated Absorbance in the Carbonyl and Carbon Double Bond Regions as a Function of Depth into Liner 2 .

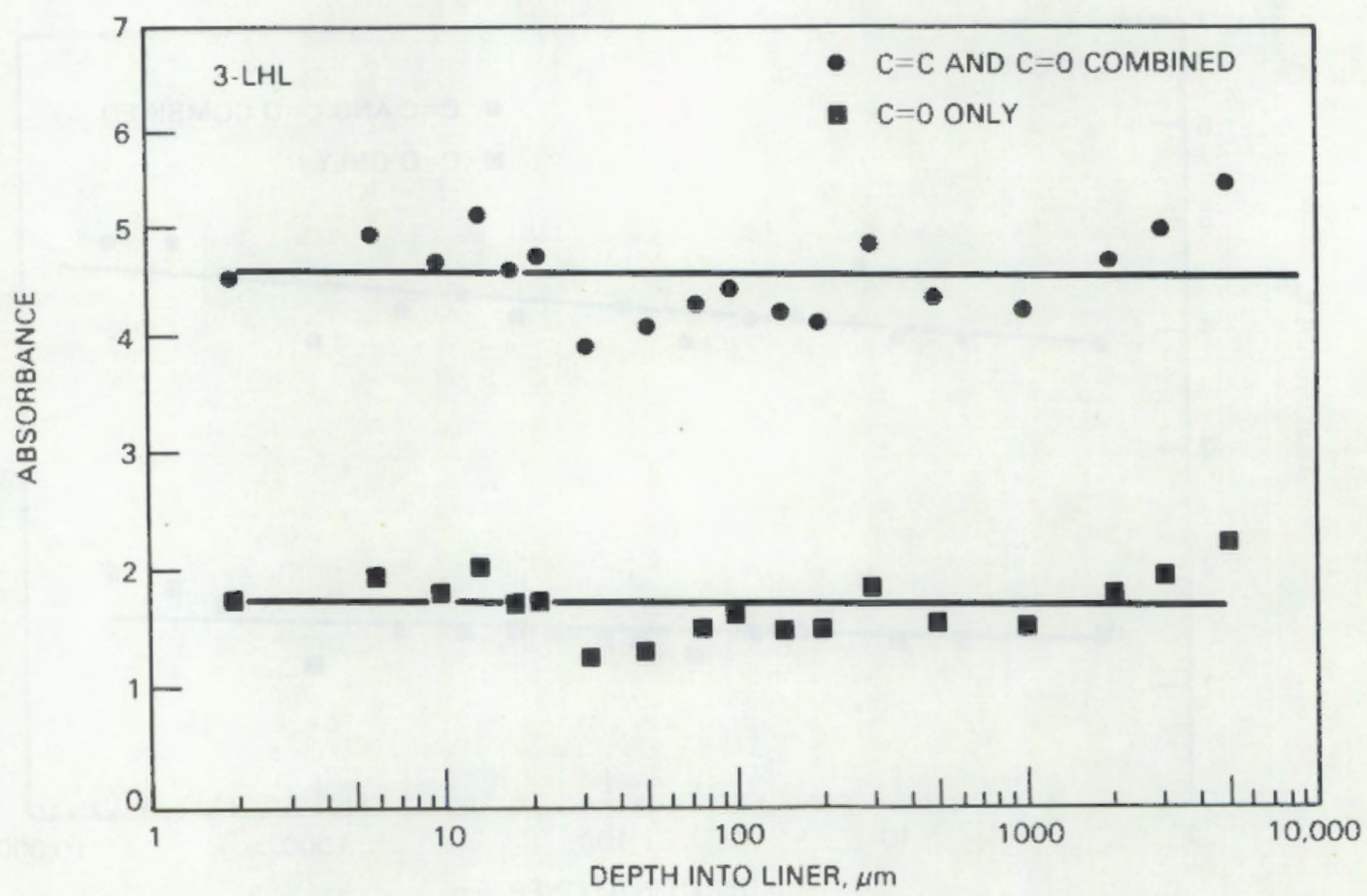

FIGURE C.26. Integrated Absorbance in the Carbonyl and Carbon Double Bond Regions as a Function of Depth into Liner 3 


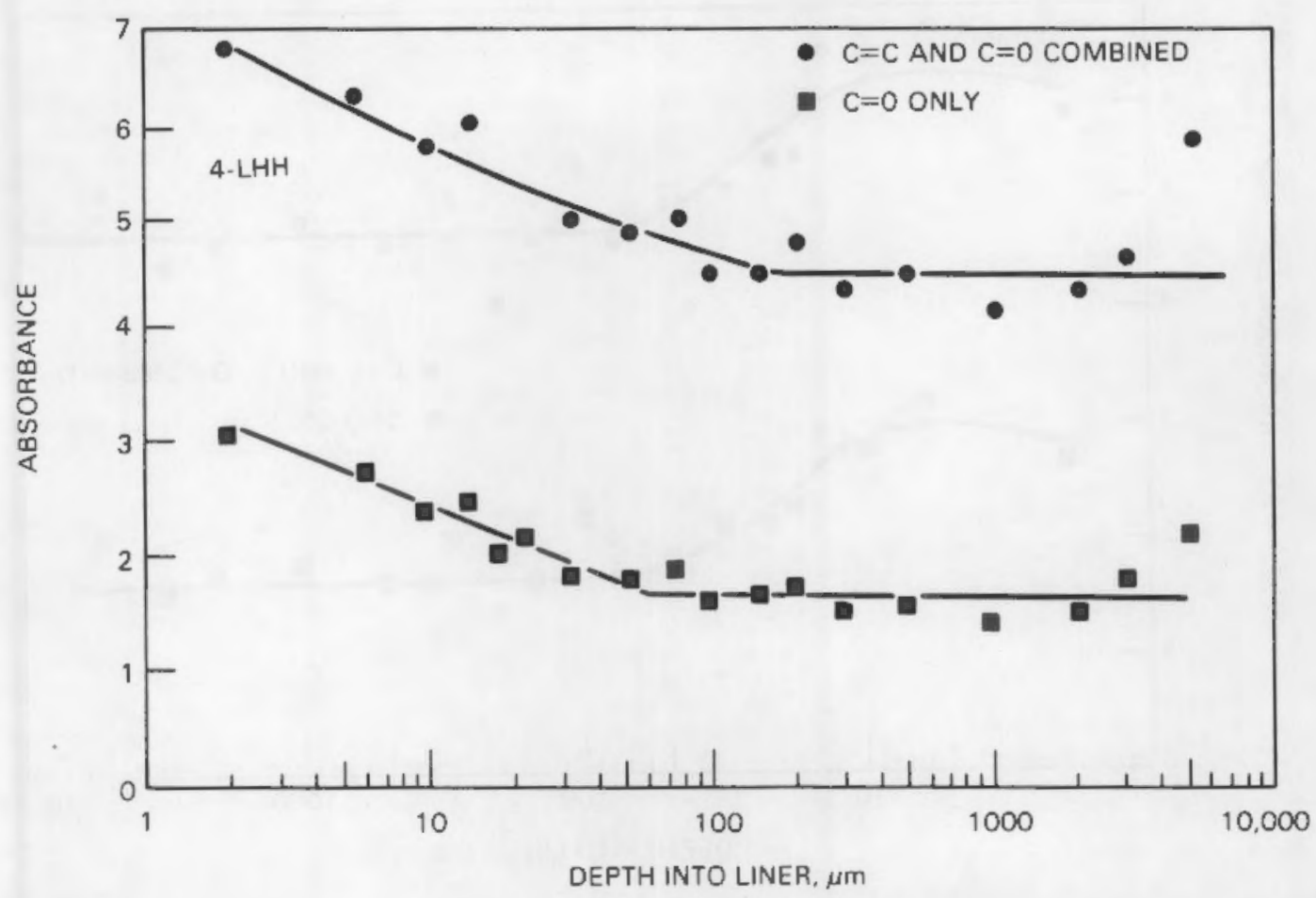

FIGURE C.27. Integrated Absorbance in the Carbonyl and Carbon Double Bond Regions as a Function of Depth into Liner 4

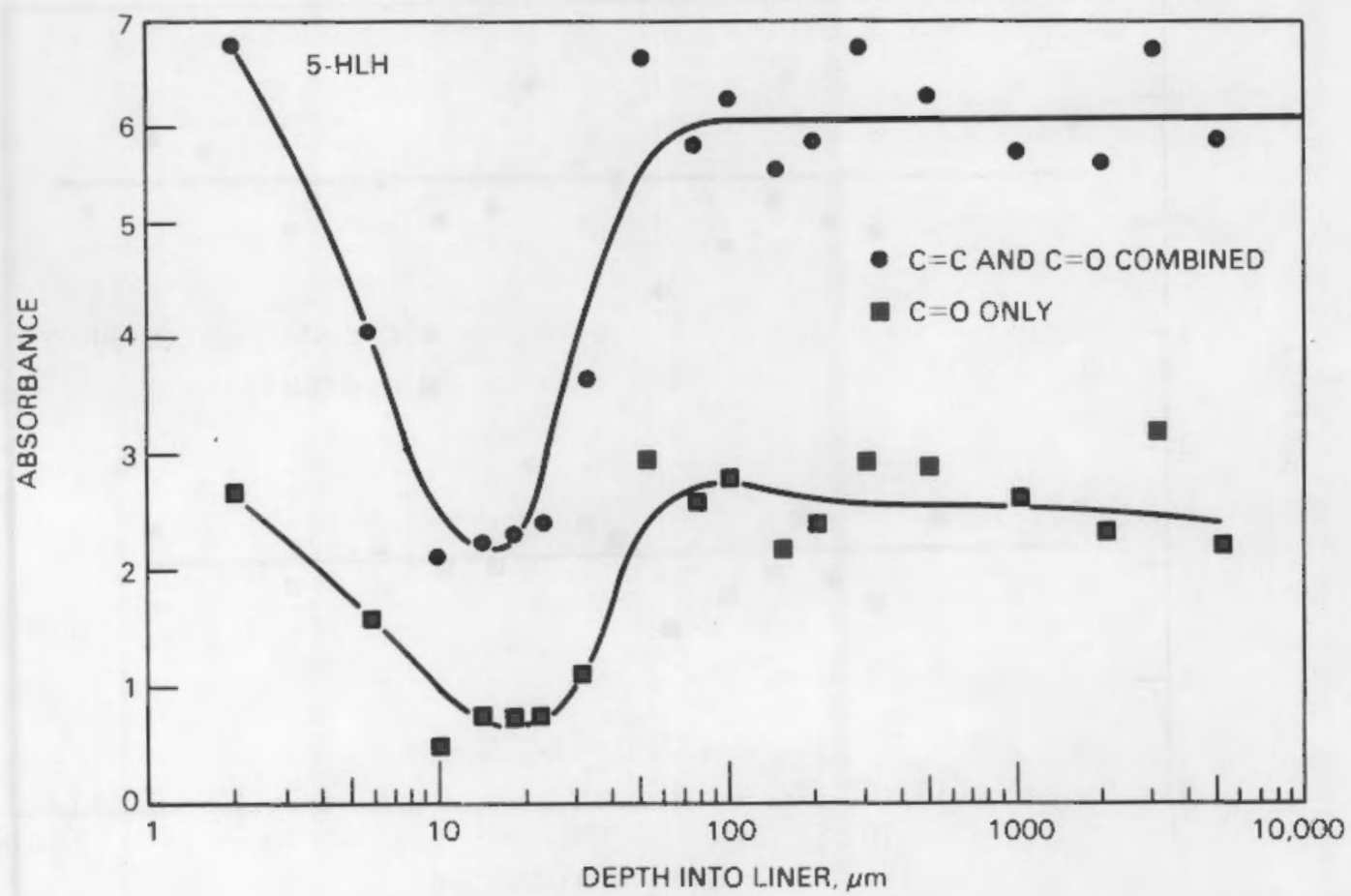

FIGURE C.28. Integrated Absorbance in the Carbonyl and Carbon Double Bond Regions as a Function of Depth into Liner 5 


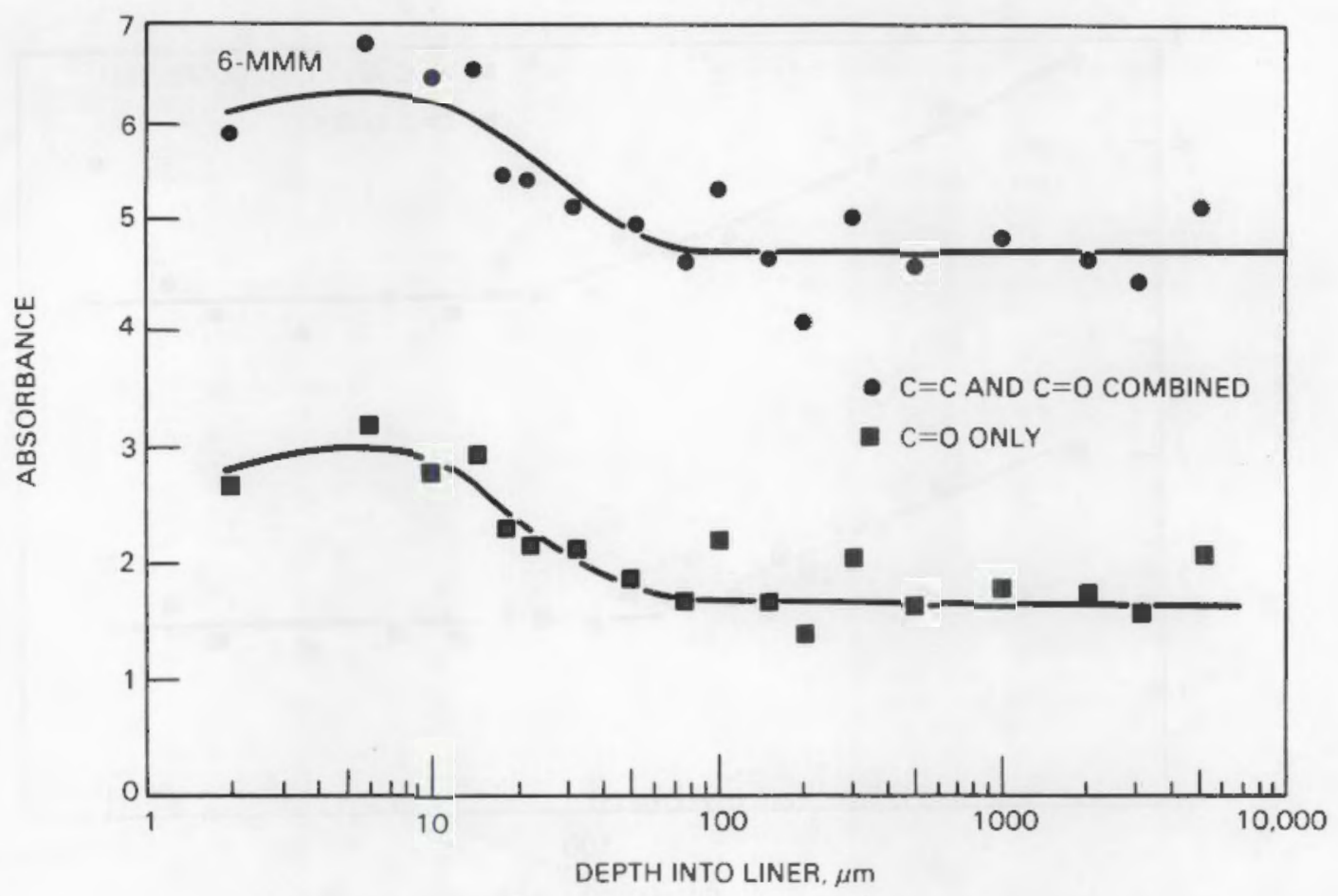

FIGURE C.29. Integrated Absorbance in the Carbonyl and Carbon Double Bond Regions as a Function of Depth into Liner 6

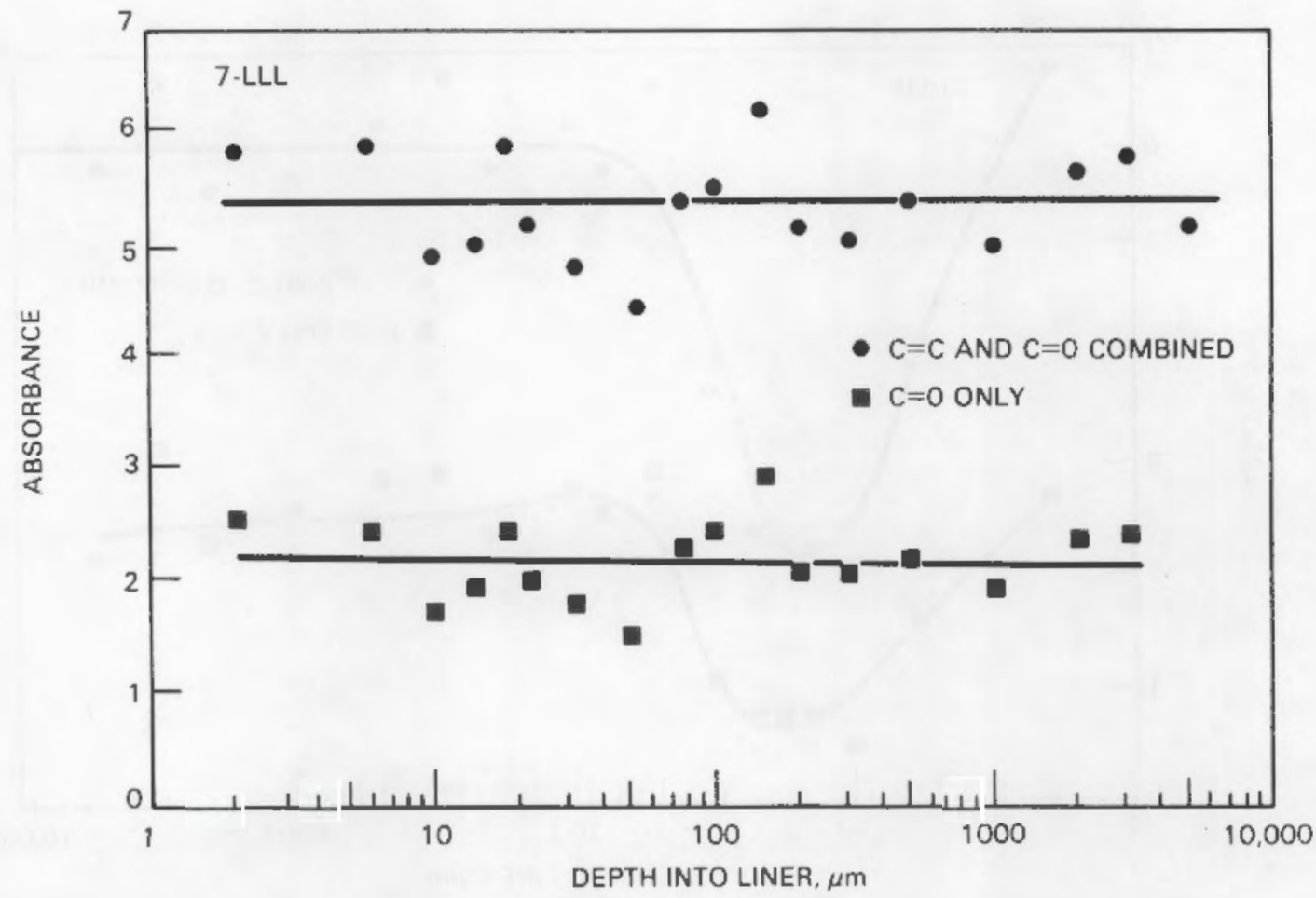

FIGURE C.30. Integrated Absorbance in the Carbonyl and Carbon Double Bond Regions as a Function of Depth into Liner 7 
DOE /UMT -0216

PNL -4842

UC-70

\section{DISTRIBUTION}

No. of

Copies

OFFSITE

27 DOE Technical Information Center

William E. Mott, Director

Environmental Safety Engineering Division

U.S. Department of Energy, EP-14

Washington, DC 20545

J. E. Baublitz, Director

Division of Remedial Action Projects

0 ffice of Nuclear Waste Management

U.S. Department of Energy, NE-301

Washington, DC 20545

E. Delaney

Division of Remedial Action Projects

Office of Nuclear Waste Management

U.S. Department of Energy, NE-301

Vashington, DC 20545

D. H. Groelsema

Division of Remedial Action Projects Office of Nuclear Waste Management U.S. Department of Energy, NE-301 Washington, DC 20545

A. Kluk

Division of Remedial Action Projects Office of Nuclear Waste Management

U.S. Department of Energy, NE-301

Washington, DC 20545

M. G. White

Division of Remedial Action Projects

Office of Nuclear Waste Management

U.S. Department of Energy, NE-301

Washington, DC 20545
No. of

Copies
E. L. Keller, Director

Technical Service Division

DOE Oak Ridge Operation Office

P.0. Box E

Oak Ridge, TN 37830

Office of the Assistant Manager for Energy Research and Development

DOE Oak Ridge Operations office

P.0. Box E

Oak Ridge, TN 37830

J. A. Morley

Project Manager

Uranium Mill Tailings

Project office

Suite 1700

5301 Central Ave. NE

Albuquerque, NM 87108

M. L. Matthews

Project Engineer

Uranium Mill Tailings

Project office

Suite 1700

5301 Central Ave. NE

Albuquerque, NM 87108 
No. of

Copies

W. A. Nixon

Office of Nuclear Materials,

Safety and Safeguards

Mail Station 396-SS

U.S. Nuclear Regulatory

Commission

Washington, DC 20555

G. Gnugnoli

Mail Station 1130-SS

U.S. Nuclear Regulatory Comnission

Washington, DC 20555

F. Swanberg

U.S. Nuclear Regulatory Commission

Mail Station 1120-SS

Washington, DC 20555

S. Lichtman

Criteria and Standards Division

Office of Radiation Programs

Environmental Protection Agency

Washington, DC 20460

$R$. F. Overmyer

Ford Bacon and Davis

P.0. Box 8009

Salt Lake City, UT 84108

T. E. Hakonson

Los Alamos Scientific Laboratory

P.0. Box 1663

Los Alamos, NM 86544

W. E. Kislieleski

Argonne National Laboratory

9700 South Cass Avenue

Argonne, IL 60439

T. N. Narasimhan

Lawrence Berkeley Laboratory

Berkeley, CA 94720

J. D. Nelson

Professor, Program Leader

Colorado State University

Fort Collins, CO 80523
No. of

Copies

\author{
R. A. Scarano, Chief \\ Uranium Recovery License \\ Branch \\ Mail Station 483-SS \\ U.S. Nuclear Regulatory \\ Commission \\ Washington, DC 20555 \\ H. C. Barber \\ Jacobs Engineering \\ Group, Inc. \\ Suite 1700 \\ 5301 Central Ave. IIE \\ Albuquerque, NM 87108 \\ K. R. Krishnan \\ Program Manager \\ Jacobs Engineering \\ Group, Inc. \\ Suite 1700 \\ 5301 Centrl Ave. NE \\ Albuquerque, NM 87108
}

$20 R$. Petersen

Document Control

Jacobs Engineering Group, Inc.

Suite 1700

5301 Central Ave. NE

Albuquerque, NM 87108

D. A. Emilia

Advanced Technology Division

Bendix Field Engineering Corp.

P.0. Box 1569

Grand Junction, CO 81502

0. D. Markham

Radiological and

Environmental Sciences

Labora tory

P.D. Box 2108

Idaho Falls, ID 83401 
No. of

Copies

F. W. Wicker

Radiology Radiation

Biology Department

Colorado State University

Fort Collins, C0 80521

Battelle Memorial Institute

Beverly Rawles

Office of Nuclear Waste Isolation

505 King Avenue

Columbus, $\mathrm{OH} 43201$

ONSITE

5. Richland Operations office

0. J. Elgert

H. E. Ransom

J. J. Schreiber

M. Shupe

J. D. White

7 Rockwell Hanford Operations

V. Q. Hale

D. L. Lane

H. E. McGuire

J. Patterson

S. J. Phillips

J. F. Relyea

S. A. Weigman
No. of

Copies

\author{
G. Markos \\ Research Insti tute for \\ Geochemistry and \\ Environmental Chemistry \\ 2693 Commerce Road \\ Rapid City, SD 57701 \\ V. C. Rogers \\ Rogers \& Associates \\ Engineering \\ P.0. Box 330 \\ Salt Lake City, UT 84110
}

ONSITE

39 Pacific Northwest Laboratory

S. M. Barnes

W. F. Bonner

J. L. Buelt (20)

T. D. Chikalla

M. E. Dodson

M. G. Foley

G. H. Gee

M. S. Hanson

J. N. Hartley

P. L. Koehms tedt

R. E. Nightingale

J. V. Robinson

D. J. Silviera

Technical Information (5)

Publishing Coordination (2) 


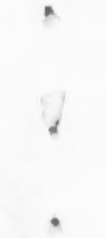

